\title{
The neuropharmacology of attentional modulation in primate visual cortex
}

\author{
Dissertation \\ for the award of the degree \\ "Doctor rerum naturalium"
}

Division of Mathematics and Natural Sciences

of the Georg-August-Universität Göttingen

submitted by

Vera Katharina Veith, née Marks

from Münster

Göttingen 2016 
Doctoral thesis

committee:

\author{
Prof. Dr. Stefan Treue (Advisor, First Refree) \\ Cognitive Neuroscience Laboratory \\ German Primate Center (DPZ) \\ Prof. Dr. Hansjörg Scherberger (Second Refree) \\ Neurobiology Laboratory \\ German Primate Center (DPZ) \\ Prof. Dr. Melanie Wilke \\ Department of Cognitive Neurology \\ Universitätsmedizin Göttingen \\ Prof. Dr. Tobias Moser \\ Institut für Auditorische Neurowissenschaften \& \\ InnenOhrLabor \\ Universitätsmedizin Göttingen \\ PD Dr. Peter Dechent \\ Abteilung Kognitive Neurologie \\ Universitätsmedizin Göttingen \\ Prof. Dr. Siegrid Loewel \\ Systems Neuroscience Group \\ Universität Göttingen
}

Date of the oral examination: 04.03.2016 
Herewith I declare that I have written this thesis independently and with no other aids and sources than quoted.

Göttingen, 29.01.2016

Vera Katharin Veith 


\section{Acknowledgements}

This work would not have been possible without support.

First of all, I would like to express my deepest gratitude to my advisor, Prof. Dr. Stefan Treue, for his excellent guidance, patience, caring and providing me with an excellent atmosphere for doing research. Besides my advisor, I would like to thank the rest of my thesis committee: Prof. Dr. Hansjörg Scherberger and Prof. Dr. Melanie Wilke, for their insightful comments and valuable suggestions during the committee meetings.

For help with taking care of the monkeys, for providing expert support as well as speedy solutions for all kind of technical problems I would like to thank Sina Plümer, Leonore Burchardt, Klaus Heisig and Dirk Prüsse. Beatrix Glaser I thank for all the kind help in administrative work. Additionally I would like to thank Ralf Brockhausen for his computer and technical support.

I am also thankful to my current office mates Cheng Xue, Suresh Krishna and Veera Katharina Menz and my former office mates Philipp Ulbrich and Kevin Windolph for sharing everyday lab life, inspiring discussions and support whenever needed.

A special thanks to Cliodhna Quigley for her great team work in the neuropharmacology project, for her enormous support in electrophysiology recording, monkey handling, data analysis as well as for her great advice for thesis writing.

I would also like to thank Shubhodeep Chakrabarti, Valeska Stephan, Antonio Calapai and Cliodhna Quigley for proofreading of this thesis and to Pablo Martinez Vazquez for his excellent advises and support. 
I would also like to thank Philipp Ulbrich, Farina Bubert, Jan Lause and Vighneshvel Thiruppathi for recording part of the data for my psychophysics experiments.

I am enormously grateful to my family for their loving and unlimited support providing me with a strong encouragement.

Last but not least I would like to thank my husband Johannes. I am so blessed to have you, as true companionship is hard to find, someone to share my ups and downs. You are my peace of mind. 


\section{Contents}

INTRODUCTION

MOTIVATION AND OVERVIEW OF THIS THESIS

OVERVIEW OF THE INTRODUCTION

MOTION PROCESSING IN THE VISUAL SYSTEM

THE HIERARCHICAL ORGANIZATION OF THE PRIMATE VISUAL CORTEX 4

VISUAL MOTION PROCESSING

AREA MT 8

$\begin{array}{ll}\text { Attention } & 10\end{array}$

$\begin{array}{ll}\text { VISUAL ATTENTION } & 11\end{array}$

$\begin{array}{ll}\text { SPATIAL ATTENTION } & 13\end{array}$

$\begin{array}{ll}\text { Perceptual characteristics of spatial attention } & 13\end{array}$

$\begin{array}{ll}\text { Physiological characteristics of spatial attention } & 16\end{array}$

$\begin{array}{ll}\text { ACETYlCholine } & 18\end{array}$

$\begin{array}{ll}\text { ACETYLCHOLINE - A NEUROMODULATOR } & 18\end{array}$

$\begin{array}{ll}\text { CHOLINERGIC RECEPTOR TYPES } & 19\end{array}$

$\begin{array}{ll}\text { THE ANATOMY OF THE CHOLINERGIC SYSTEM } & 21\end{array}$

CHOLINERGIC PROJECTIONS TO THE CORTEX 23

CHOLINERGIC FEEDBACK PROJECTIONS TO THE CORTEX 25

MODELS TO EXPLAIN ACETYLCHOLINE ACTION IN THE CORTEX 26

THE CHOLINERGIC HYPOTHESIS

$\begin{array}{ll}\text { AtTENTIONAL MODULATION AND ACETYLCHOLINE } & 31\end{array}$

LINKING ATTENTIONAL MODULATION TO CHOLINERGIC FUNCTION -

TECHNICAL LIMITATIONS

ATTENTIONAL MODULATION CAN BE DIRECTLY LINKED TO ACETYLCHOLINE FUNCTION 33

VI VS. MT - RECEPTOR CONTRIBUTION TO ATTENTIONAL MODULATION 
HUMAN LINEAR VISUAL MOTION DIRECTION DISCRIMINATION THRESHOLDS: GRADED DEPLOYMENT OF SPATIAL ATTENTION SHOWS HEMIFIELD DEPENDENT RESOURCES

HUMAN LINEAR VISUAL MOTION DIRECTION DISCRIMINATION THRESHOLDS: EFFECTS OF GRADED DEPLOYMENT OF SPATIAL ATTENTION AND SIGNAL STRENGTH

A PRESSURE INJECTION SYSTEM FOR INVESTIGATING THE NEUROPHARMACOLOGY OF INFORMATION PROCESSING IN AWAKE BEHAVING MACAQUE MONKEY CORTEX

THE ROLE OF THE CHOLINERGIC SYSTEM IN ATTENTIONAL MODULATION IN AREA MT OF THE PRIMATE VISUAL CORTEX 


\section{Introduction}

\section{Motivation and overview of this thesis}

Visual attention is the key feature for the visual system to filter relevant information that enters the system via the retina. Scientific interest in visual attention has increased within the last 25 years. Especially within the last decade, the information gained from multiple studies has broadened the horizon of attention-induced influences on a physiological and psychophysical level. However, the source of attentional control is to a large extent unknown and reported effects of attention are ambiguous.

This thesis aims to shed light on the mechanisms of spatial attention, a selective process allowing prioritizing spatial locations in the visual field, on a psychophysical as well as on a perceptual level.

The first and the second manuscript address the psychophysical aspects of spatial attention. Here, I investigated the influence of varying magnitudes of spatial attention on human subjects' performance in discriminating visual motion directions. With the chosen stimuli I aim to target area MT, an area in the extrastriate cortex thought to underlie the perception of linear motion. As area MT is involved in early stages of cortical processing, I was targeting bilateral vs. unilateral visual field differences in the first manuscript. In the second manuscript I was interested to equate the effects of attention with the effects of signal strength and to measure their individual as well as their interactive influence on perceptual performance. 
The third and the fourth manuscript address physiological aspects, in particular the neuropharmacology of spatial attention. The focus of the third manuscript lies on the methodological approach of pressure injection. This method allows a reversible pharmacological manipulation of the direct vicinity of the recording electrode and therefore exerts temporary influence on the local neurotransmitter network. Using this method, I investigated the cholinergic contribution to spatial attention modulation in the macaque monkey medial temporal area MT. The study described in manuscript four is the first study investigating the cholinergic involvement in attentional modulation in area MT of an awake, performing macaque monkey.

\section{Overview of the introduction}

This introduction provides a global overview of the main components of this thesis. It introduces the visual area of interest, mechanisms of spatial attention with regard to physiological and psychophysical effects, and the neuromodulator acetylcholine, a candidate for regulating the selective process of attention.

In detail, the first part will give an introduction to visual information processing by highlighting its hierarchical organization. Motion processing is here of particular interest.

Attention will be introduced in a second step, especially the psychophysical as well as physiological characteristics of visual attention directed toward spatial locations.

In the third part of the introduction the neuromodulator acetylcholine is described. The anatomy of the cholinergic system in the central nervous system is introduced, including its topographically organized projections to the cortex. 
The variety of cholinergic receptor types is highlighted, as well as their distribution in the pre- and post synapse and the variety of effects these receptors can trigger in the cortex. Additionally, the most recent models explaining the action of acetylcholine in the central nervous system are introduced, as well as the cholinergic hypothesis of several brain functions and dysfunctions.

The fourth section will describe and discuss various studies reporting different aspects of cholinergic involvement in attention and highlight their limitations to directly link attentional modulation to specific cholinergic contribution. Specifically, recordings from striate cortex of macaque monkeys, which measured the direct relationship between acetylcholine and attentional modulation for the first time, will be described. In the last part of the introduction I will motivate the main study of this thesis, investigating the neuropharmacology of attentional modulation in area MT in the context of visual motion processing. 


\section{Motion processing in the visual system}

\section{The hierarchical organization of the primate visual cortex}

Visual information processing in primates is achieved via a hierarchy of visual cortical areas, which show individual specifications to analyze certain features. For the visual system, two main anatomically separated, complementary processing streams, the ventral and the dorsal stream, have been hypothesized to be involved in processing different aspects of the visual scene (Ungerleider and Mishkin, 1982). The ventral stream is anatomically organized along the occipito-temporal cortical pathways originating in primary visual cortex and projecting via $\mathrm{V} 2, \mathrm{~V}_{4}$ to IT and TEO. The dorsal stream also arises in the primary visual cortex and is located along the occipito-parietal pathway, where information is forwarded via V2 and V3 to area MT and MST (Van Essen and Maunsell, 1983). Higher areas of the dorsal stream are area FST, LIP and VIP.

In addition to an anatomical segregation, the two processing streams can be distinguished based on their functional specificity. The ventral stream subserves recognition and distinction of object features like color and shape, whereas the dorsal stream shows highest specificity for the spatial aspects of visual scenes and plays therefore a major role in visual motion processing. Although the dorsal and ventral streams are anatomically and functionally distinctive and this two-streams hypothesis is widely accepted, there are additional anatomical interconnections linking areas of both streams (Ungerleider and Desimone, 1986). This supports a functional connectivity that goes beyond the two-stream model of neural processing. 
Although there are clear distinctions between the two processing streams, they also share common features. The early visual cortical areas in each of the two streams process simple and localized visual information, whereas higher visual cortical areas respond to more complex aspects of visual information (DeYoe and Van Essen, 1988).

In addition to an increasing preference for stimulus complexity, the neuronal receptive fields show continuous enlargement with every successive stage in the hierarchy (Van Essen and Maunsell, 1983). A receptive field of a neuron is defined as a region in the visual field, in which a stimulus elicits a response of that neuron. Neurons in early visual area V1 have very small receptive field sizes of one visual degree, whereas receptive fields of neurons in late visual areas, like IT, cover a relatively large area of the visual field including the fovea (Gross et al., 1972).

As the main focus of this thesis lies on the dorsal stream and especially on processing of visual motion it will be described here in more detail.

\section{Visual motion processing}

As motion perception is an essential feature of every organism in order to navigate through the environment and to detect mates, prey and predators, it is no surprise that visual areas exist, that are specialized for encoding motion. In the macaque monkey, the main areas involved in motion processing are the medial temporal area MT, the medial superior temporal area MST and the fundal superior temporal area FST, all being part of the dorsal stream (Zeki, 1974, Orban et al, 1995, Boussaoud et al., 1990).

However, it is also known that in macaque neocortex, the origin of motion selective processing is layer $4 \mathrm{~B}$ of the primary visual cortex $\mathrm{V} 1$ (Hubel and Wiesel, 1968, Mishkin and Ungerleider, 1982). In 1968, it was first 
demonstrated by Hubel and Wiesel that neurons in area V1 of the macaque monkey show directional selectivity: an increase in response to a specific direction of motion, while showing little or no response to the opposite motion direction (Hubel and Wiesel, 1968).

There is a direct connection from area V1 to area MT via a subset of highly specialized, directionally selective, spiny stellate neurons (Maunsell and van Essen, 1983). These V1 neurons already show direction tuning. Thus MT doesn't appear to compute the directional information exclusively de novo (Movshon and Newsome, 1996). In addition, there is also an indirect input from pyramidal neurons in area V1 to MT via the thick stripes of area V2 and through area V3 (Ponce et al., 2008). This indirect input mainly serves for binocular-disparity segregation (Hubel and Livingstone, 1987), whereas propagation of motion processing to area MT is merely secondary. Figure 1 depicts the main visual areas being involved in motion processing in the macaque monkey.

However, it was demonstrated that available motion information in area MT does not solely arise from area V1, as it was demonstrated that area MT showed residual motion responses after a V1 lesion (Rodman et al., 1989). Motion information is thought to additionally originate from the colliculo-cortical pathway and a combined lesion of the superior colliculus and V1, in contrast, completely abolishes directional responses in MT (Rodman et al., 1990). 


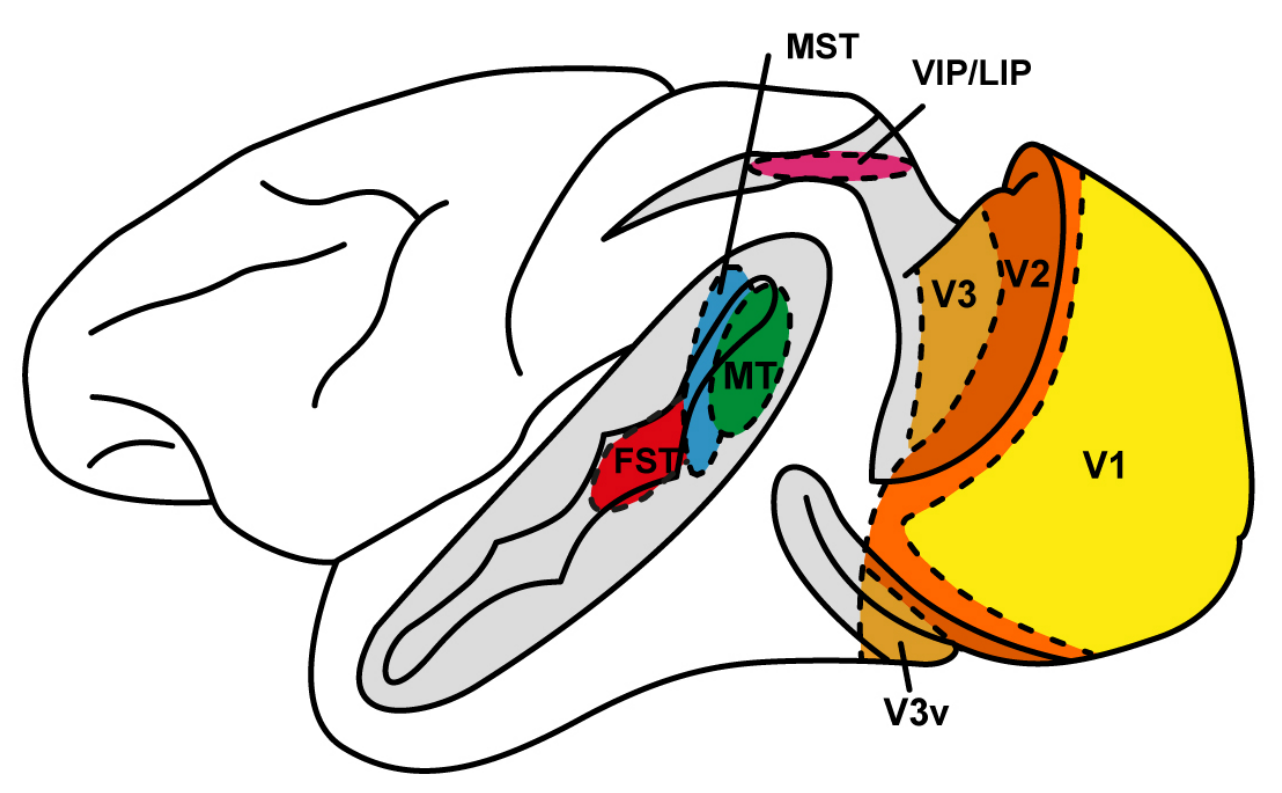

Figure 1: A schematic view of the main visual cortical areas involved in motion processing in the macaque monkey. The superior temporal sulcus, intraparietal sulcus, lunate sulcus and inferior occipital sulcus are presented open (grey shaded areas) in order to provide a better view of the areas of interest. V1-V3: early visual areas, V3v: ventral part of $\mathrm{V}_{3}$ area, MT: medial temporal area, MST: medial superior temporal area, FST: fundal superior temporal area, VIP: ventral intraparietal area, LIP: lateral intraparietal area. Adapted from Parker et al. 2007.

Visual motion information is directly transmitted from area MT to MST (Maunsell and van Essen, 1983), where even more complex aspects of motion are computed. Area MT is sensitive for linear movement direction and speed components (Albright, 1984), whereas the dorsal region of MST demonstrates selectivity to rotating, expanding and translational constituents of optic flow motion stimuli (Duffy and Wurtz, 1991). Additional higher-level areas that are involved in motion processing are the ventral intraparietal area VIP, lateral intraparietal area LIP, and the fundal superior temporal area FST (Colby et al., 1993, Fanini and Assad, 2009, Rosenberg et al., 2008). 


\section{Area MT}

The medial temporal area MT with its location in the posterior bank of the superior temporal sulcus (STS) was first described more than 40 years ago (Dubner and Zeki, 1971). Anatomically, it can be clearly defined based on its heavily myelinated structure (Hof and Morrison, 1995). Functionally, it can also be distinguished from other visual areas based on the sensory properties of its neurons (Dubner and Zeki, 1971).

Decades of experiments have contributed to this area being one of the bestunderstood areas in terms of its sensory properties. Attributes like speed selectivity (Maunsell and van Essen, 1983), selectivity for motion in depth (Albright et al., 1984) as well as sensitivity to chromatic signals (Dobkins and Albright, 1994) have been ascribed to MT. The medial temporal area is present in each hemisphere and contains the representation of the entire contralateral visual field. It inherits its retinotopic organization from visual area V1 and thereby shows a highly systematic topography (Dubner and Zeki, 1971, Albright and Desimone, 1987). The receptive field size of MT neurons growths as a linear function of eccentricity and is 10-fold larger compared to the receptive fields of the direct input visual area V1 (Gattass and Gross, 1981, Albright and Desimone, 1987).

The majority of neurons in area MT (60-100\% depending on the chosen stimuli) show strong tuning for the direction of visual motion in their receptive field, whereas fewer V1 neurons show a response to moving stimuli as well as a narrower tuning bandwidth (Albright et al., 1984, Albright, 1984). Interestingly, MT neurons form a columnar structure for direction of motion, similar to the columnar organization for orientation in area V1 (Newsome and Salzman, 1993, Albright, 1984). In Figure 2, a cartoon visualizes the columnar structure of area MT. 


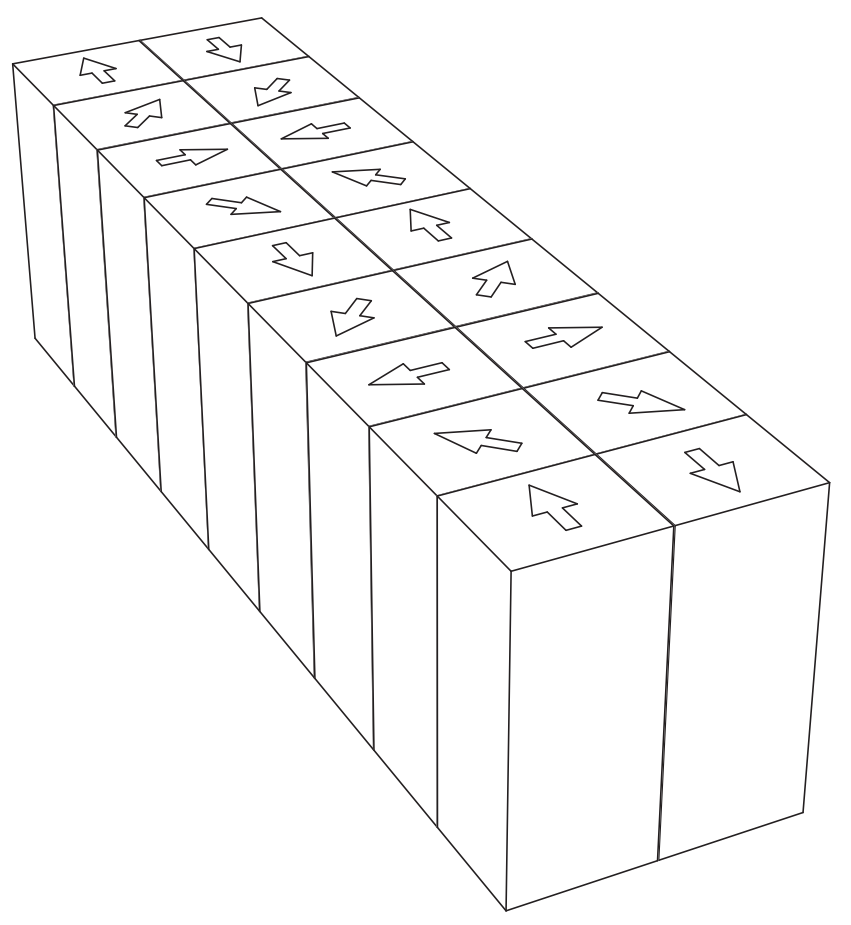

Figure 2: A cartoon showing the simplified columnar structure of a section of the medial temporal area (MT). Neurons in each column show highest response to one motion direction, illustrated by arrows. This preferred motion direction changes systematically within one row covering angles from o - 360 degree. Across rows, neighboring columns show opposite direction preference. The height represents the cortical depth. This diagram is based on data and the corresponding figure in Albright et al. (1984).

Despite the central role of area MT in the encoding of the sensory properties of visual motion stimuli, the activity of MT neurons also reflects a number of other influences, that modulate the firing rate of a given MT neuron to a moving stimulus, even if that stimulus remains unchanged in its motion parameters.

The three main classes of such modulatory influences are stimulation history, bottom-up and top-down influences. The stimulation history determines the level and type of adaptation (Saul \& Cynader, 1989), whereas the bottom-up influence describes a change in firing rate of a neuron due to changes in the 
physical characteristics of the stimulus e.g. luminance contrast (Skottun, 1987). The main top-down influence on neural activity is the allocation of attention. Here voluntary allocation of attention to a certain feature or a specific location in the visual field leads to a change in firing rate (Treue and Maunsell, 1996). Each one of the three classes has been shown to exert a multiplicative effect, i.e. a gain change on neuronal responses in area MT. In the next section I will focus on the top-down influence of attention.

\section{Attention}

Why do we need attention? We are surrounded by and have to deal with a huge amount of sensory information, but our resources are limited. It was calculated, that the amount of information entering our optic nerves is in the range of 10 Million bits/second (Koch et al., 2006). This is comparable with the transmission rate of an Ethernet connection. Processing this entire amount of information would exceed the possible energy consumption of the brain and would go beyond the limits of cognitive resources. For that reason a selective process in the brain is required to decide which information is behaviorally relevant and should be processed further.

What is attention? Although the word "attention" is an integral component of our vocabulary and is used by us on a daily basis in different contexts, we are often not aware of its importance and detailed meaning. Attention cannot be easily described by a simple definition or explained by synonyms as it has a manifold impact on all sensory processes. This is reflected by the vast amount of adjectives, which are used to classify attention, such as visual, auditory, complex, focused, split, overt, covert, top-down, bottom-up, object-based, feature-based, space-based, voluntary, involuntary, limited, sustained, transient... 
However, William James formulated the best applicable definition describing attention in 1890: "Everyone knows what attention is. It is taking possession of the mind, in clear and vivid form, of one out of what may seem several simultaneously possible objects or trains of thoughts... It implies withdrawal from some things in order to deal effectively with others, and is a condition which has a real opposite in the confused, dazed, scatterbrained state which in French is called distraction, and Zerstreutheit in German."

Today, one definition, which captures the essence of William James' words, would be that attention is a selective modulation of sensory information processing, guided by behavioral relevance. More detailed information regarding the involvement of attention in the visual system will be given in the following section.

\section{Visual attention}

Visual attention is the key feature for the visual system to filter relevant information that enters the retina and enhances its processing. Interest in studying visual attention has increased within the last 25 years and has improved our understanding (Carrasco, 2011). This success is due to the availability of new and improved imaging methods like functional magnetic resonance imaging (fMRI), magnetoencephalography (MEG), event-related potentials (ERP) or steady-state visual evoked potentials (SSVEP). All those methods pave the way to an insight into the brain, while it is involved in a task that demands attentional deployment. Other approaches like human psychophysics have also been improved with the availability of high-resolution eye trackers, allowing precise monitoring of eye movements, pupil diameter etc., that have been shown to be correlates of attention (Lisi et al., 2015). This made it possible to reliably disentangle eye movements from attentional deployment (Cornelissen et al., 2002). Electrophysiological recordings in the macaque 
monkey (Treue, 2001) and computational modeling (Itti and Koch, 2001) add the missing components to a wide-ranging approach to visual attention.

The effects of visual attention are measurable on various levels. On a behavioral level, it was shown that paying attention to a stimulus reduces reaction time (Posner et al., 1980), improves accuracy (Carrasco and McElree, 2001), alters appearance (Carrasco et al., 2004), enhances spatial resolution (AntonErxleben and Carrasco, 2013) and alters perception of spatial properties such as size, shape and spatial frequency of an object (Anton-Erxleben et al., 2007, Fortenbaugh et al., 2011, Abrams et al., 2010). Additionally, with the usage of imaging techniques, it was shown that attention causes a consistent and systematic increase in brain activity of visual areas (Gandhi et al., 1999), as it was shown to add a baseline shift to fMRI responses (Runeson et al., 2013). Furthermore, seminal neurophysiological studies investigating visual attention have enabled the identification of various brain areas involved in the process of attention, showing an increase in firing rate, when a behaviorally relevant stimulus is presented in the receptive field of the recorded neuron (Treue and Maunsell, 1996).

Where in the visual cortex is attentional modulation present? Attention leads to a change in neuronal response in every visual cortical area examined (Maunsell and Cook, 2002). For a long time it was believed that only high-level visual areas can be attentionally modulated, whereas others were ascribed as purely sensory. Within the last two decades this assumption could be disproved, as it was demonstrated that also neurons in early visual areas such as area MT (Treue and Maunsell, 1996) as well as the primary visual cortex V1 (Gandhi et al., 1999) showed attentional modulation. Therefore, it is assumed that a 'pure' sensory processing area does not exist in the primate visual cortex (Treue, 2003). In addition, it was shown that an even earlier brain area - the first relay between the eye and the cortex, the lateral geniculate nucleus (LGN) - shows 
attention-induced increase in response (McAlonan et al., 2008). Although every component of the visual cortex is influenced by attention, it could be shown that the higher the visual area is located in the cortical hierarchy, the bigger the average response enhancement by attention (Maunsell and Cook, 2002). The magnitude of attentional modulation doesn't solely depend on the involved visual areas, as it additionally depends on the type of visual attention that is executed. There are various distinctive, but not exclusive (Soto and Blanco, 2004), forms of visual attention including spatial attention, object-based attention, and feature-based attention.

In feature-based attention a certain stimulus feature, e.g. movement direction or color, is behaviorally relevant, independent of its location. Object-based attention describes a selection process that is guided by object content, whereas in spatial attention the attentional selection targets spatial locations in the visual field. The concept of spatial attention will be described in more detail, as it is the form of visual attention investigated in this thesis.

\section{Spatial attention}

\section{Perceptual characteristics of spatial attention}

Spatial attention is a selective process allowing subjects to prioritize spatial locations in the visual field and therefore enhance their processing. Psychophysically, spatial attention has been shown to enhance behavioral performance. Behavioral responses to an attended location are faster (e.g. Posner, 1980); vision is of higher spatial resolution (Yeshurun and Carrasco, 1998) and has enhanced sensitivity for fine changes (Carrasco et al, 2004). Unattended stimuli in turn appear to be lower in contrast or might not be noticed at all (Carrasco, 2006, Reynolds \& Chelazzi, 2004). In psychophysical tasks, pre-cues provide information about the behaviorally relevant location. This information in turn is known to drive attentional mechanisms (Carrasco, 
2011; Pashler and Johnston 1998). When this information is diminished or not provided at all, meaning that no location is selected for preferential processing, a behavioral cost is observed in the form of a decrement in perceptual performance (Posner, 1980). This phenomenon is often attributed to the limited capacity of spatial attention (Kahneman, 1973, Broadbent, 1971). However, several psychophysical studies were not able to distinguish whether the measured improvement in behavioral performance is ascribed to a reduction in stimulus uncertainty or to an actual improvement in information processing (Pashler, 1994, Pashler and Johnston, 1998). These studies may therefore fail to detect the true attention effect (Lou and Maunsell, 2015). In the case of simple visual performance, such as direction discrimination, the tasks are seen to have low attentional cost (Braun and Julesz, 1998). It is assumed, that there is some amount of visual awareness outside the attentional spotlight, permitting the subject to perform at both locations, inside and outside of the spotlight, with equal performance for stimuli with low attentional cost (Braun and Julesz, 1998). This observed lack in attentional improvement for simple movement discrimination is contradictory to the physiologically measured improvement on a cellular level (Luo and Maunsell, 2015). In this thesis, I designed an innovative paradigm in order to be able to investigate true spatial attention effects and circumvent the effects of stimulus uncertainty on to our results. Additionally, I gradually manipulated the deployment of spatial attention, by introducing pre-cues of varying validity. Details about the task design are described in manuscript one and two of this thesis.

Furthermore, spatial attention was attributed with various metaphors aiming to illuminate its underlying mechanism. The 'spotlight' metaphor, proposed by Michael Posner in 1980, describes a cone of light targeting one specific area in space, while all the remaining areas are kept in 'darkness'. He conducted a spatial cuing paradigm to guide the subjects' spatial attention, independent of eye movements, called covert spatial attention. He showed that information on spatial position improves reaction time, whereas detection of stimuli located 
outside of the spotlight incurs a temporal cost. In addition, he demonstrated that this spotlight could be shifted independent of eye movements (Posner, 1980). In general, this spotlight of attention allegory implies that only one region in space can be attended at one time, is fixed in its diameter and therefore has to be shifted across the visual field when more than one spatial location should be attended. A variation of the 'spotlight' metaphor is the 'zoom lens' metaphor implying adaptable mechanisms that act like a zoom lens, increasing or decreasing in diameter based on perceptual demands (Eriksen and St James, 1986). One important hypothesis shared by both models is, that there is only one attentional focus. Pylyshyn and Storm challenged the assumption of one spotlight by convincingly showing continuous tracking of multiple independently moving targets (Pylyshyn and Storm, 1988). Over the years more and more evidence has arisen challenging the assumption of one attentional spotlight in favor for multiple non-continuous attentional foci (Morawetz et al., 2007, Castiello and Umiltà, 1992, Cavanagh and Alvarez, 2005).

In addition, multiple studies aim to investigate even more specific characteristics relating time and location of spatial attention. For example it could be demonstrated that an additional sudden distractor onset destroys the goal-directed focus of attention (Kramer and Hahn, 1995). Alvarez and Cavanagh proposed, that spatial attention distribution, in the context of attentional tracking, is independently limited in the left and right visual hemifield (Alvarez and Cavanagh, 2005). They could show, that twice as many targets can be tracked when the targets are presented across hemifields, as when they are presented only in one hemifield.

Both psychophysical experiments, conducted within this thesis, support the multifocal distribution of spatial attention in the context of linear movement discrimination. The results gained in the second study additionally support the notion of two independently active attentional resources, one being active in each hemifield. 


\section{Physiological characteristics of spatial attention}

Physiologically, spatial attention leads to an increase in neuronal firing rate when the attentional focus matches a neuron's receptive field (Treue and Maunsell, 1999). In a classical physiological setup, single unit activity from macaque visual cortex is measured and compared while the monkey either deploys attention to a stimulus placed inside the neuron's receptive field or to a similar stimulus outside of it. The deployment of attention is done covertly, requiring the monkey to fixate a centrally presented spot and to switch its attentional deployment in the absence of eye movements. The stimuli presented are chosen to match the known feature selectivity of the recorded neuron, e.g. coherently linearly moving dots for area MT. Neuronal response is then characterized with the neural tuning curve, a plot of the average firing rate as a function of diverse values of a certain stimulus feature (Butts and Goldmann, 2006)(see Figure 3). Early studies investigating the attentional modulation on tuning curves describe sharpened selectivity for attended stimuli (Spitzer et al., 1988), whereas later studies do not confirm attention-induced change in selectivity (Treue and Martinez-Trujillo, 1999, McAdams and Maunsell, 1999). They reported a multiplicative change in neural tuning curve, showing a proportional neuronal response enhancement for all values presented, without affecting the width of the tuning curve (see Figure 3), termed response gain model. 


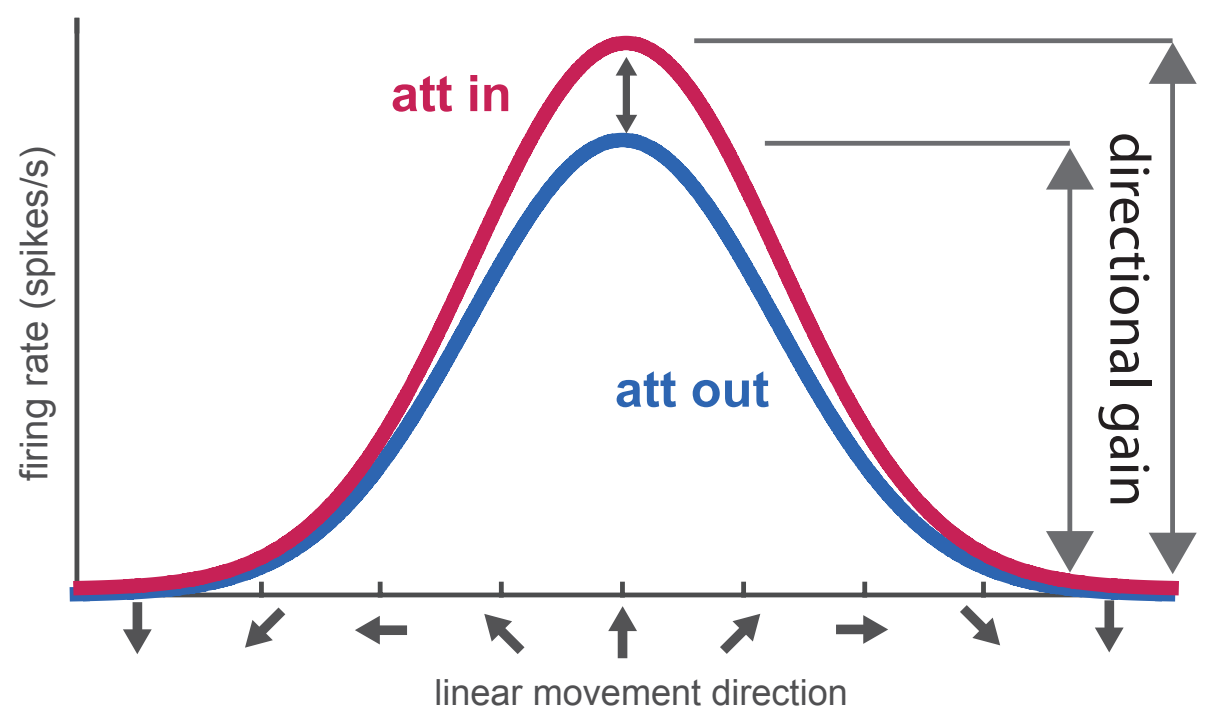

Figure 3: Multiplicative effect of attention in area MT. The cartoon depicts a Gaussian shaped tuning curve of an MT neuron with highest response to upward stimulus direction. When attention is directed into the neurons receptive field (att in, red curve), the neuron's response is enhanced by a fixed factor for all stimuli directions relative to when attention is directed outside of receptive field (att out, blue curve).

In distinction to the response gain model stands the contrast gain model, describing attentional effects on stimuli with different contrast values. This model proposes a leftward shift of the contrast response function towards lower contrast values when attention is deployed (Reynolds et al., 2000, MartinezTrujillo and Treue, 2002). It could be shown that the activity pattern of the two models is not exclusive. Based on their results, Hermann and colleagues proposed, that depending on stimulus size and attention field size, attention modulates the activity in visual cortex, resembling either a change in response gain or contrast gain (Hermann et al., 2010). 


\section{Acetylcholine}

In 1936, Otto Loewi and Sir Henry Dale were awarded the Nobel Prize for their explorations on chemical neurotransmission and especially for the discovery of the vagusstoff acetylcholine (Rubin, 2007). Beside the initially found activity in the frog's vagus nerve (Loewi, 1924) and the exhibition of physiological activity in vertebrate organs (Dale, 1929), acetylcholine (ACh) is highly involved in a broad spectrum of activities in the central nervous system. Below I will focus on the action of ACh in the brain, as it is the main emphasis of my thesis.

\section{Acetylcholine - a neuromodulator}

Based on studies on the peripheral nervous system at neuromuscular junctions and on the autonomic nervous system, ACh is seen as a classic, fast-acting neurotransmitter. However, this activity pattern could rarely be shown in the central nervous system (Changeux, 2010), where acetylcholine's activity is mostly seen in a neuromodulator fashion. A neuromodulator is understood as a neurotransmitter that can diffuse over long distances, having influence on multiple neurons and synapses on a timescale of seconds, minutes or even hours. Neuromodulators mainly act on G-protein coupled receptors and alter excitatory as well as inhibitory transmission. This type of neural communication is termed volume or diffuses transmission (Zoli et al., 1999).

This slow action in the CNS is in contrast to the extremely fast-acting mechanisms of the enzyme acetylcholinesterase (AChE), located at the synaptic cleft. AChE functions to terminate synaptic transmission by catalyzing the breakdown of ACh. It is seen as an ideal enzyme in terms of timing properties as it approaches the upper limit allowed by diffusion of the substrate ACh as well as for catalytic mechanisms (Tougu, 2001, Taylor and Radic, 1994). 
Although the neuromodulatory characteristics of ACh in the CNS support the cholinergic action to be in a timescale of minutes or even hours, studies additionally support fast-acting characteristics of ACh in the CNS (Herrero et al., 2008). The distinctive activity pattern can be partly clarified by the existence of different receptor types with different subunit configurations, that are located at different positions in the synaptic cleft and in different brain areas (Dani and Bertrand, 2007, Picciotto et al., 2012). The following sections give an insight into the complexity of the cholinergic system, covering its anatomy, connectivity pattern, and functional components.

\section{Cholinergic receptor types}

Already in 1914, Henry Dale reported two types of cholinergic actions that vary independently (Dale, 1914). Later it was proven that acetylcholine indeed acts via two functionally different classes of receptors: metabotropic muscarinic receptors (mAChRs) and iontotropic nicotinic receptors (nAChRs) (Tatsuya, 1993, Picciotto et al., 2000). The muscarinic type, classically named after its activator muscarine (agonist), acts via second messenger cascades and therefore mediates a slow response. Within the central nervous system, five muscarinic receptors subtypes (M1-M5) have been identified. The subtypes $\mathrm{M}_{1}, \mathrm{M}_{3}$, and $\mathrm{M}_{5}$ are coupled to $\mathrm{G}(\mathrm{q} / \mathbf{1 1})$ and activate phospholipase $\mathrm{C}$, whereas the subtypes M2 and M4 are coupled to G(i/o) and inhibit adenylyl cyclase activity, regulating a variety of fundamental functions (Picciotto et al., 2000).

Nicotinic receptor subtypes, on the other hand, comprise five subunit polypeptides that can occur in heteromeric or homomeric congregations. Those receptors, named after their affinity to the tobacco alkaloid nicotine, are ligandgated ion channels and therefore mediate fast synaptic transmission. So far 16 types of genetically distinct receptor subunits have been identified, again being clustered in sub-families. There are nine alpha subunits ( $\alpha 1-9)$, four beta 
subunits $(\beta 1-4)$, one gamma $(\gamma)$, one delta $(\delta)$ and one epsilon subunit $(\varepsilon)$ (Lukas et al, 1999).

Muscarinic and nicotinic receptor types are located pre- and postsynaptically and can elicit heterogeneous effects based on their variation in location and molecular composition (see Figure 4).

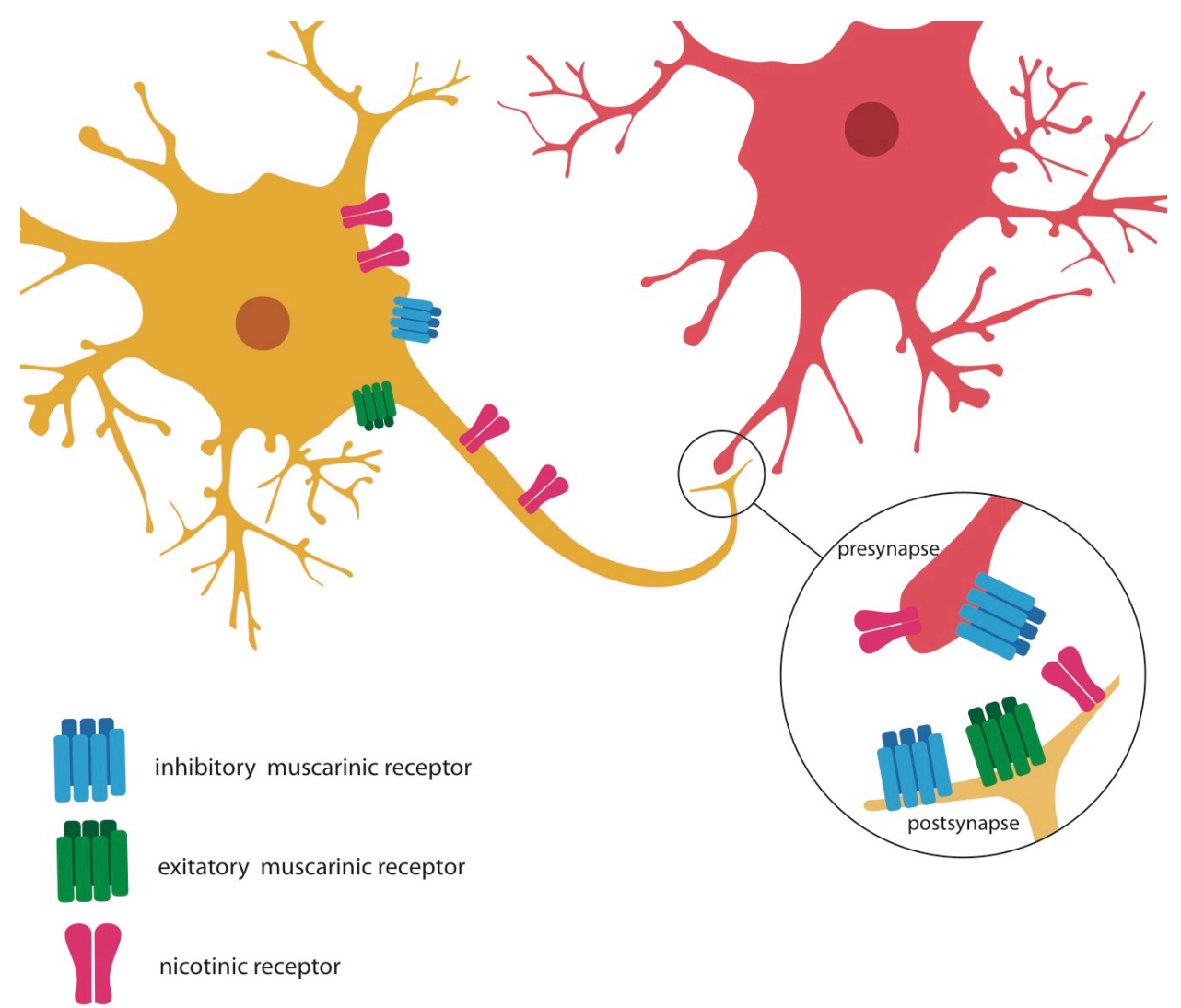

Figure 4: Nicotinic and muscarinic acetylcholine receptor distribution in pre- and post synapse. Inhibitory muscarinic AChRs are mainly found presynaptically, providing a negative feedback loop in signal transduction. Postsynaptically, inhibitory as well as excitatory muscarinic AChRs are found. Presynaptic nicotinic AChRs predominantly trigger activations of other neurotransmitters and ACh itself. Postsynaptic and nonsynaptic nicotinic AChRs increase a neuron's firing rate when activated and participate in synaptic plasticity. This figure is based on Picciotto et al., 2012. 
Presynaptic mAChRs, M2 (predominantly found in cortex) and M4, are mainly inhibitory, serving as a negative feedback loop in signal transduction. Muscarinic AChRs located at the post-synapse can be either inhibitory (M2, M4) or excitatory (M1, M3, M5) (Wess, 2003). Presynaptic nAChRs in contrast can trigger the activation of other neurotransmitters like GABA, glutamate, dopamine, serotonin etc., as well as ACh itself (McGehee et al., 1995, Picciotto et al., 2012), whereas postsynaptic or nonsynaptic nAChRs participate in synaptic plasticity, mediate excitation and activity-dependent modulation (Dani and Bertrand, 2007).

The distribution of different receptor types was shown to differ across visual cortex areas in the macaque monkey. For example, in V1 inhibitory neurons strongly express $\mathrm{m} 1$ muscarinic receptors, whereas cells in MT mainly express the $\mathrm{m} 1$ type on excitatory neurons (Disney et al., 2014).

\section{The anatomy of the cholinergic system}

In the central nervous system, $\mathrm{ACh}$ is released from two main neuronal groups of projections, innervating a broad range of cortical and subcortical sites, named the basal forebrain cholinergic system and the brainstem cholinergic system (see Figure 5). The basal forebrain cholinergic system plays an important role in aspects of attentional function, whereas the brainstem cholinergic system is an important component in regulating the sleep/wake cycle (Everitt and Robbins, 1997).

In the 1980s, with the help of histochemistry and immunohistochemistry, Mesulam and colleagues were able to subdivide the cholinergic neurons within the basal forebrain cholinergic system of the macaque monkey and the human into four, partially overlapping, major cell groups based on their connectivity pattern. The nomenclature Ch1-4 was introduced to label the choline- 
acetyltransferase-positive neurons within these four cell groups (Mesulam et al. 1983a, Mesulam and Geula, 1988).

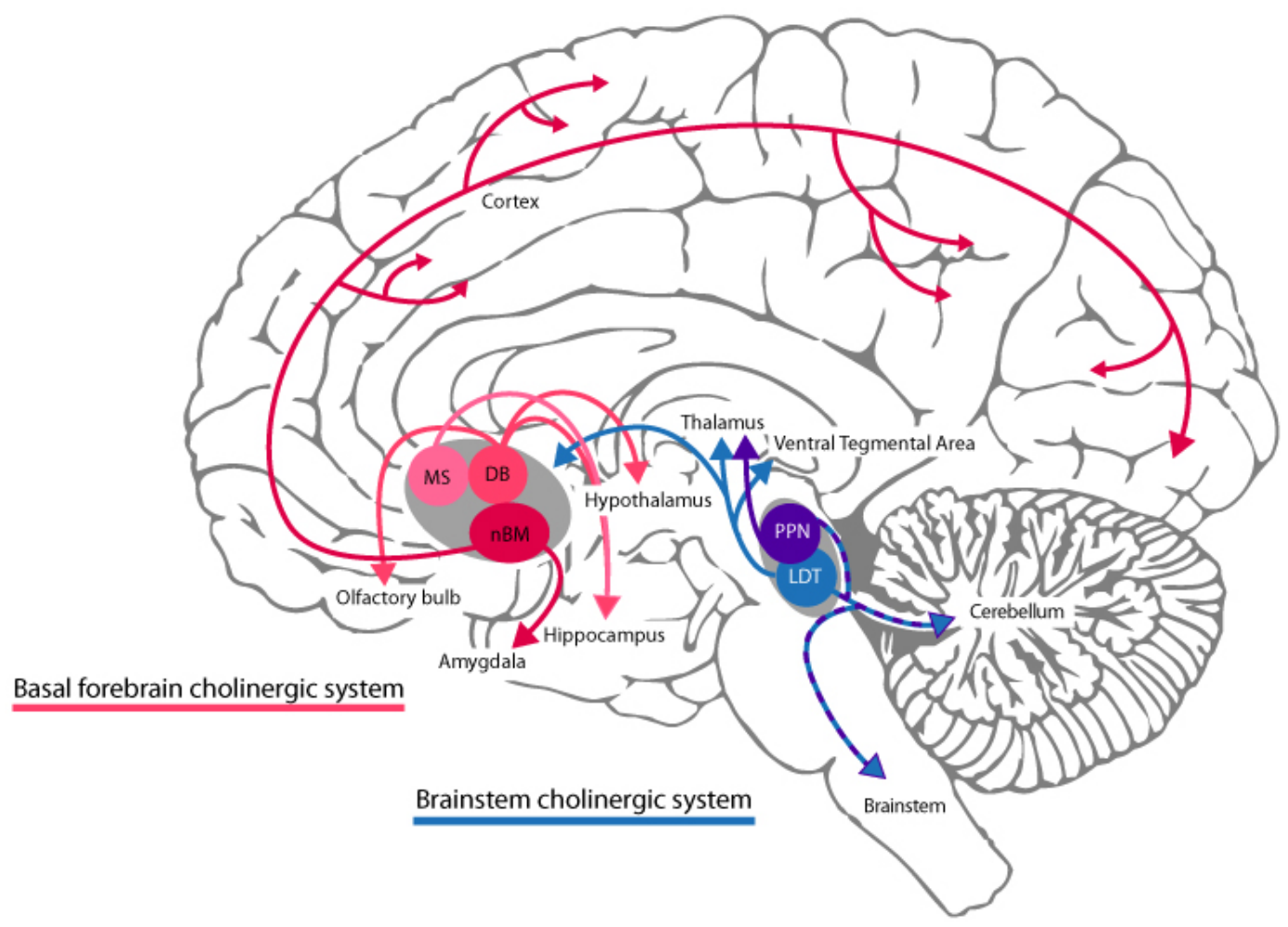

Figure 5: Cholinergic system of the human brain. Two main cholinergic sources of the central nervous system: Basal forebrain cholinergic system (red/magenta) and the brainstem cholinergic system (blue/purple). Each system is subdivided into certain cell groups, that target different brain areas and provide them with acetylcholine (arrows). For the basal forebrain cholinergic system three groups are defined: MS: medial septal nucleus, DB: ventral and horizontal nucleus of the diagonal band, nBM: nucleus Basalis of Meynert. For the brainstem cholinergic system two cell groups are defined: PPN: pedunculopontine nucleus, LDT: laterodorsal tegmental gray of the periventricular area. Based on Picciotto et al., 2012.

The Ch1 cholinergic group is located in the medial septal nucleus (MS) mainly projecting to the hippocampus (see Figure 5). Ch2 and Ch3 mostly correspond 
to the vertical and horizontal nucleus of the diagonal band (DB), targeting the hippocampus and hypothalamus or the olfactory bulb, respectively. The Ch4 group, located in the nucleus Basalis of Meynert, plays a distinct role in this context, as it forms the greatest quantity of cholinergic neurons compared to all other cholinergic sources and provides the major cholinergic resource for the entire cortex as well as additionally targeting the amygdala.

For the brainstem cholinergic system two cholinergic groups were defined (Ch5 and Ch6), based on their connectivity pattern (Mesulam et al., 1983a). The Ch5 cholinergic group is mainly found in the pedunculopontine nucleus (PPN) whereas the Ch6 group has its origin in the laterodorsal tegmental gray of the periventricular area (LDT). Both sectors provide the main cholinergic input for the thalamus, but additionally target the basal forebrain region as well as providing a minor component of the corticopetal cholinergic innervation (Mesulam et al. 1983b). Furthermore, they deliver ACh to the cerebellum and the brainstem (Perry et al., 1999).

\section{Cholinergic projections to the cortex}

The nucleus basalis of Meynert, as part of the basal forebrain cholinergic system (see Figure 5), is a highly differentiated, relatively large area and can be topographically subdivided into six main sectors: anterior-medial, anteriorlateral, anterior-intermediate, intermediate-dorsal, intermediate-ventral and posterior sub-areas (Mesulam et al. 1983b). The cortical cholinergic, acetylcholine-esterase-rich fibers provide a major cholinergic projection to the entire cortex and thereby form a topographical organization (Bigl et al., 1982; Henderson, 1981; Johnston et al., 1981) (see Figure 6). In detail, neuroanatomical studies of the monkey brain show, that anterior-medial parts of the nucleus basalis deliver the main source of ACh to the medial cortex as well as in the cingulate gyrus. Anterior-lateral parts of the nucleus mainly target 
frontal and parietal opercular regions as well as the amygdaloid nuclei. Both intermediate areas project similarly to ventrolateral orbital, insular, peristriate, inferotemporal and parahippocampal areas and to inferior parietal areas. The posterior parts of the nucleus basalis of Meynert mainly form the cholinergic source for superior temporal cortex and the temporal pole (Mesulam et al., 1983b).

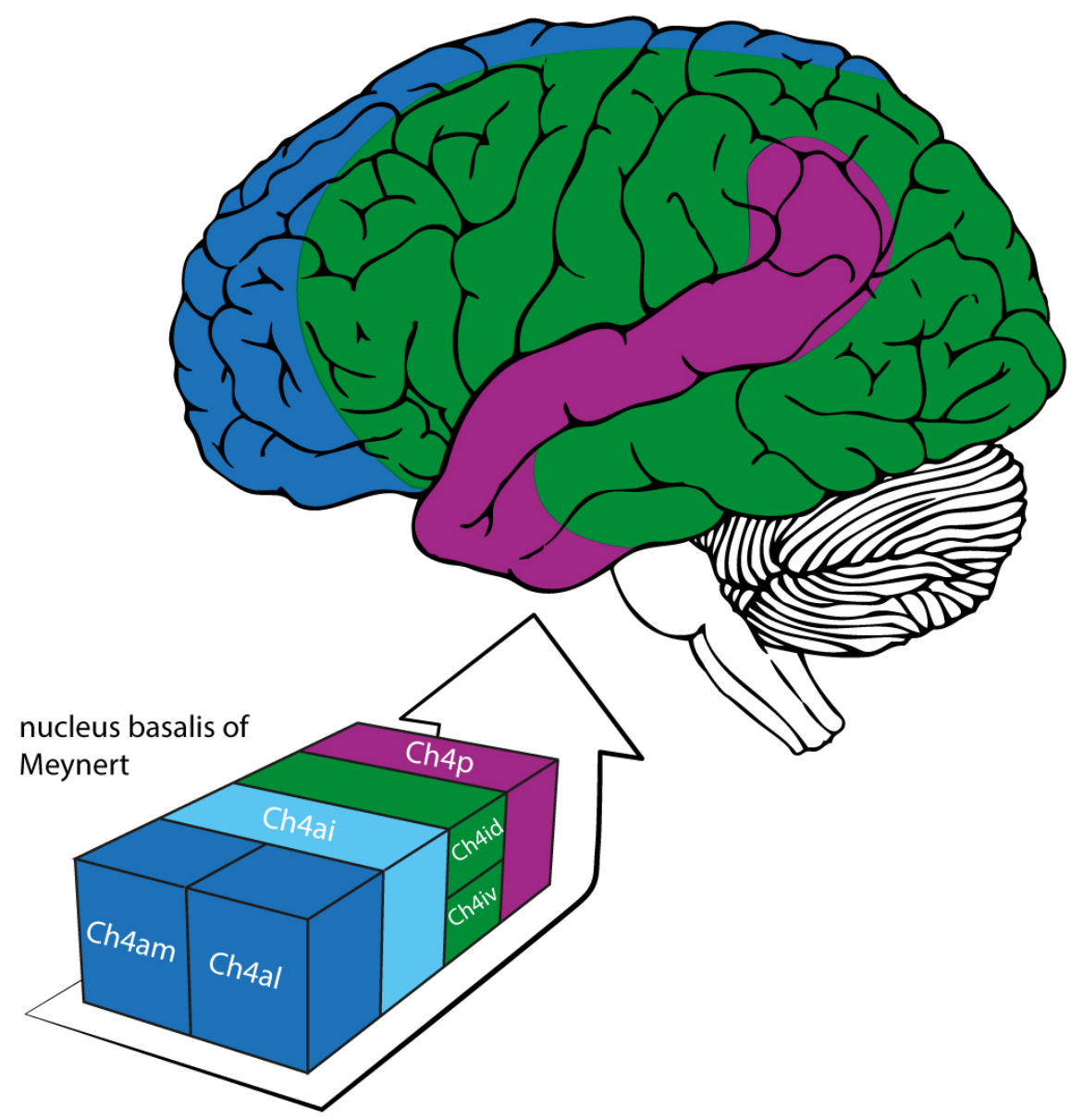

Figure 6: Cholinergic projections from the nucleus basalis of Meynert to the cortex in the human brain are highly topographically organized. Anterior areas of the nucleus basalis of Meynert (Ch4am, Ch4al) are shown in blue color, intermediate areas (Ch4id, Ch4iv) in green and the posterior part (Ch4p) in pink. The projections from the anterior part Ch4ai (light blue) are unknown. The targeted cortical areas are colored correspondingly. For a detailed description see text. This Figure is based on Mesulam et al., 1983 a,b and Liu et al, 2015. 
The cholinergic projection neurons form clusters providing a widespread but topographically organized network of cholinergic innervation and can thus be seen as analogous to the organization of serotonin neurons in the dorsal raphe nucleus (Van Bockstaele et al., 1993) or to the nucleus locus coeruleus (Loughlin et al., 1986).

Beside the cholinergic projection neurons, a second group of cholinergic neurons has been discovered: the cholinergic interneurons. Those intrinsic cholinergic neurons are tonically active. They are located in the striatum and the nucleus accumbens (Eckenstein and Baughman, 1984; Benagiano et al., 2003; Doig et al., 2014). Additionally, they have been found in the cortex as small bipolar neurons, predominantly in cortical layer 2 and 3 (Houser et al., 1985). Anatomical studies show high similarity across species, including the macaque monkey (Disney et al., 2006).

Independent of cholinergic cell type, acetylcholine is synthesized in the cytoplasm by choline acetyltransferase (CAT) and transported into vesicles by a vesicular acetylcholine transporter. ACh vesicles can be found throughout the whole neuron of cholinergic neurons, but show the highest concentration in axon terminals, where ACh is released via exocytosis to perform neurotransmission in the target areas (Witthaker, 1988).

\section{Cholinergic feedback projections to the cortex}

The whole cortex receives its cholinergic input mainly from cholinergic neurons located in the nucleus basalis of Meynert, called Ch4 neurons (Mesulam, 1983a). Tracer studies in monkeys show that not all cortical areas project back to $\mathrm{Ch} 4$. For example there are no projections found from parietal cortex, peristriate cortex, lateral temporal cortex and dorsolateral prefrontal targeting the Ch4 complex (Mesulam, 2013). In addition, projections from the hypothalamus and the amygdala enter the feedback circuit by targeting $\mathrm{Ch} 4$ 
cholinergic neurons (Mesulam and Mufson, 1984, Price and Amaral, 1981). Cortical cholinergic transmission can be potentially locally regulated via these anatomically defined circuitries, providing a putative regulative mechanism for attentional modulation.

Although there is a detailed anatomical picture present from rodent, rhesus monkey as well as from human, the underlying regulating mechanisms triggering the release and action of acetylcholine in the central nervous system are still only poorly understood.

\section{Models to explain acetylcholine action in the cortex}

Several models aim to account for the actions of ACh in the central nervous system and especially in the cortex. For example $\mathrm{Yu}$ and Dayan proposed that the neuromodulator ACh interacts synergistically and antagonistically with norepinephrine and plays a major role in the brain's implementation of uncertainty computations (Yu and Dayan, 2005). In their model, ACh is thought to signal expected uncertainty, with increases proposed to predict the unreliability of a predictive cue in a known context.

Another model describes the involvement of ACh in the enhancement of signal processing mediated by attention. It proposes that cortical cholinergic activity is a result of two interacting recruitment mechanisms: the 'signal-driven modulation of detection' and the 'top-down modulation of detection' (Sarter et al., 2005). Based on experimental data it is hypothesized that the cortical cholinergic system is a mandatory component in mediating healthy attentional performance, as it has been shown to be active when attention is deployed (Arnold et al., 2002, Passetti et al., 2000). In addition, an increase in cortical cholinergic activation could be correlated with sensory input processing (Chiba et al., 1999). Sarter and colleagues aim to incorporate these two cholinergic recruitment modes into one model. Their model is based on the assumption that 
the prefrontal cortex regulates the activity of cortical cholinergic inputs to the cortex. It is proposed that the prefrontal efferent projections either directly or indirectly target the posterior parietal cortex via the basal forebrain cholinergic system, thereby forming the 'top-down modulation of detection'. It is consequently proposed, that the cholinergic recruitment of ascending projections is modulated by attention. The signal-driven modulation of detection is seen to solely originate from basal forebrain projection.

Another model focuses on the prefrontal cortex (PFC) as a central coordinating point, mediating signal detection by glutamatergic-cholinergic interaction (Hasselmo and Sarter, 2011). The main components of this model are visualized in Figure 7. This model further proposes two separate populations of cholinergic neurons with distinct influences on the PFC. The first group of cholinergic neurons (ACh1) triggers the release of glutamate from neurons located in the medial dorsal thalamic nucleus to the PFC. These glutamatergic neurons in turn activate the second group of cholinergic neurons (ACh2), whose transient release of ACh is proposed to lead to enhanced attentional orientation and cue detection. In more detail, they propose that pre-attentive information about the signal is propagated via glutamatergic neurons originating in the medial dorsal thalamic nucleus (MD) targeting the PFC. The origin of this information is proposed to be in sensory areas and is propagated via the thalamic reticular nucleus (TRN) to the MD (Guillery et al., 1998). The exact mechanisms biasing information processing of MD projections are not yet understood, but it is known, that the basal forebrain cortical cholinergic system influences MD glutamatergic input to PFC and thereby triggering cue detection (Hallanger et al., 1987). The induced glutamatergic transient is seen to target ionotropic glutamatergic receptors located at cholinergic terminals of ACh2 neurons in PFC, triggering a cholinergic transient. 


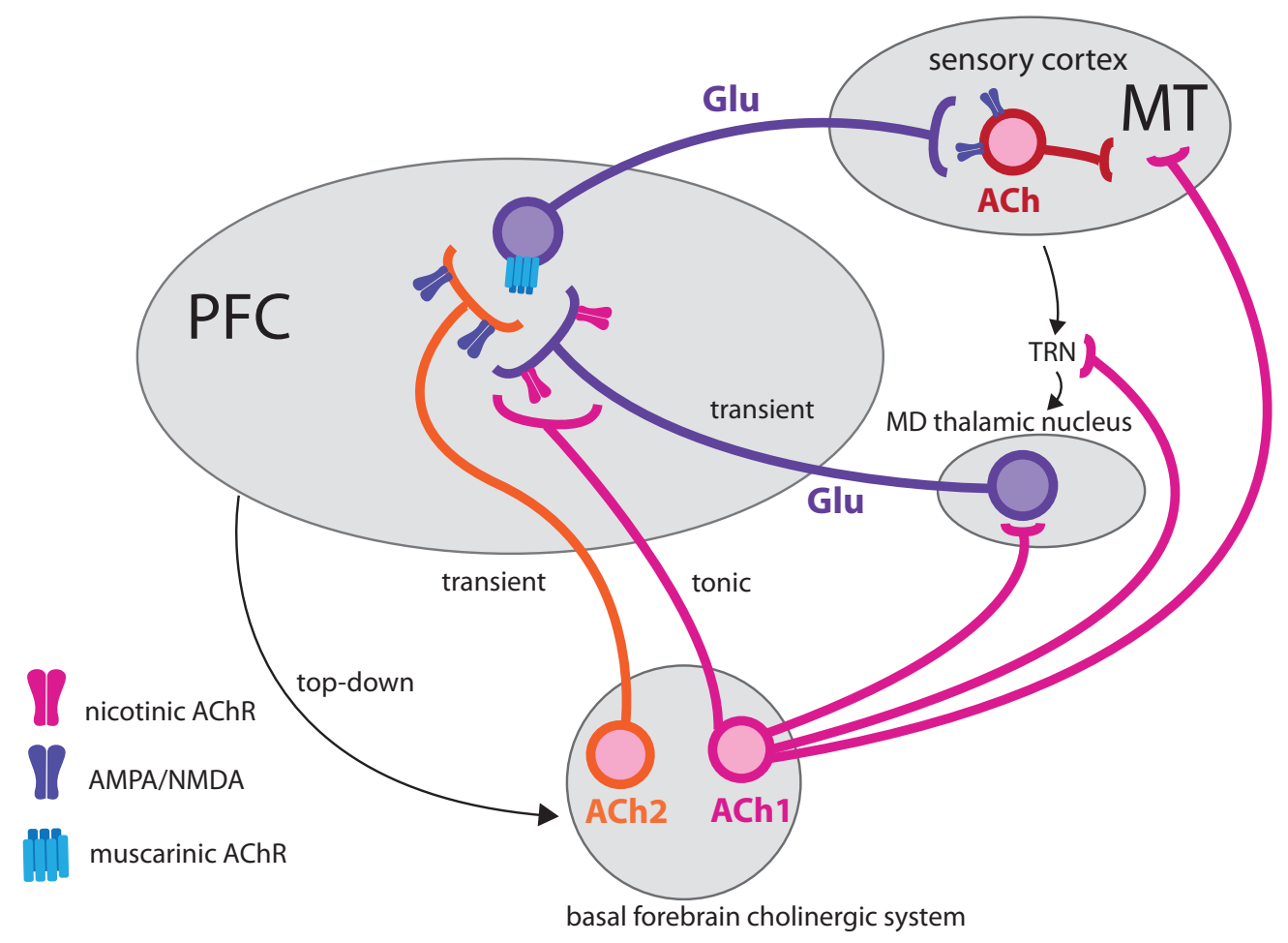

Figure 7. Global cholinergic recruitment model. In this model, the prefrontal cortex is seen as the main coordination point, which is influenced by two types of cholinergic cells (ACh1 and ACh2), both originating in the basal forebrain cholinergic system. ACh1 cells trigger the release of glutamate from glutamatergic neurons (Glu) located in the medial dorsal thalamic nucleus. The released glutamate in turn binds to the second group of cholinergic neurons (ACh2) in prefrontal cortex, inducing a transient release of acetylcholine. Acetylcholine binds to muscarinic receptor types located on glutamatergic neurons and induces an increased cholinergic release in sensory areas, such as area MT. For a more detailed description see text. This figure is based on Hasselmo and Sarter, 2011.

In addition, based on experimental data, the model assumes that different receptor types trigger different mechanisms. Whereas nicotinic receptors are seen to be involved in glutamatergic-cholinergic interactions, mediating attentional orienting and cue detection, the muscarinic type is seen to be involved in top-down controlled situations like attentional performance. For 
example it could be demonstrated that a stimulation of muscarinic AChRs in the PFC increased ACh release in parietal cortex (Nelson et al., 2005).

Although this model already incorporates many specific cholinergic effects, such as specific cholinergic projections and various receptor subtypes, it only provides a coarse and global picture of the cholinergic involvement in attentional modulation. The activity pattern of the cholinergic system in the cortex seems to be much more complex than initially thought, showing very specific, highly localized and fast regulating mechanisms on the one hand, and long term and global changes on the other hand.

Our understanding of the cholinergic system, with its regulative and functional mechanisms, is rudimentary and the models proposed therefore cover just a subset of its mechanisms.

\section{The cholinergic hypothesis}

As cholinergic neurotransmission plays a central role in cognitive abilities (Paul et al., 2015), the "cholinergic hypothesis" was formulated for several brain functions and dysfunctions, including depression, schizophrenia, and Down syndrome (Mineur and Picciotto, 2010, Tani et al., 2015, Fodale et al., 2006).

In particular, the cognitive deficits characterizing Dementia and Alzheimer's disease have been linked to a diminished amount of cholinergic fibers from basal forebrain to cortex and hippocampus, and an associated loss of cholinergic neurotransmission (Francis et al., 1999). This down-regulation in cholinergic fibers was shown to be correlated with neuronal loss in nucleus basalis of Meynert. The greatest loss was found in the intermediate and posterior part (80-88\%), which provides the main cholinergic source for the visual cortex (Mesulam, 1988). Additionally, a strong relationship between nAChRs expression and neuropathological key features was reported for Alzheimer's 
disease (Wevers et al., 2000). Further support comes from the successful treatment of cognitive deficits with acetylcholine esterase inhibitors in animals and humans. Those medications stop the activity of the fast-acting enzyme in the synaptic cleft, hydrolyzing ACh. The induced increase in ACh could be shown to reduce cognitive deficits (Bentley et al., 2003).

Although it is still under discussion whether cortical cholinergic neurons constitute the most significant contribution to the cognitive decline seen in various diseases, it is accepted that the cholinergic system plays at least a subpart (Auld et al., 2002, Geula et al., 1989) in the context of mediating specific cognitive functions like memory (Hasselmo and Stern, 2006), learning (Yu and Dayan, 2002) and attention (Sarter et al, 2005, Hasselmo and Sarter, 2010). 


\section{Attentional modulation and}

\section{Acetylcholine}

Attention induces an increased representation of attended objects on a neuronal level (Treue and Maunsell, 1996). This effect is highly consistent and reliable across studies, but the exact neurotransmitter contribution triggering this effect is largely unknown.

Cholinergic enhancement is signal-driven but has additionally been shown to be involved in top-down driven modulation like attention (for more details see section "Models to explain ACh action in cortex"). Several studies highlight the prefrontal cortex as a top-down regulator inducing either direct or indirect cholinergic release in cortical regions via the basal forebrain cholinergic system (Sarter et al., 2005).

Although both, the anatomical circuit of acetylcholine and attentional effects on a cellular level, are separately well studied, it remains a challenge to link these two components to create a full picture describing the local cholinergic mechanism of attention. Reliably manipulating attentional deployment in a suitable animal model, like the macaque monkey, requires a reliable task. In order to observe a clear and strong effect of attention on a neuronal level, it is furthermore a clear advantage to record from a mid-level visual area. Additionally, the cholinergic manipulation should fulfill certain requirements: it should be precise in spatial as well as temporary aspects. In this thesis, I aim to fulfill these criteria by choosing a spatial attention task that has previously been shown to elicit strong responses and attentional modulation of single unit activity in area MT. In addition, I used pressure injection (see manuscript 3 for details) to mimic cholinergic cortical release in a local manner. 


\section{Linking attentional modulation to cholinergic function - technical limitations}

Investigating the role of $\mathrm{ACh}$ release in complex cognitive behaviors is still challenging, given the limitations of the available techniques.

Functional magnetic resonance imaging in human has been used to link a nicotine-induced increase in response in cortical areas with attention-related cortical enhancement (Kumari et al., 2003). Due to the coarseness of the spatial and temporal resolution of this method, a direct link between attentional modulation and cholinergic function still cannot be assessed in detail, especially because the nicotinic manipulation was systemic.

Studies in rodent show that induced cell loss in the nucleus basalis of Meynert the main cholinergic source for the cortex - results in reduced cholinergic efflux in the medial frontal cortex, as measured by in-vivo microdialysis. This cholinergic reduction could be induced in a gradual manner by targeting different amounts of cells, and also correlated with a gradual attentional deficit in the rodents (McGaughy et al., 2002). In addition, studies performed by Arnold and colleagues support the high correlation between attention and ACh as they could show that demands on attentional performance selectively activate the basal forebrain cholinergic system (Arnold et al., 2002). Moreover, increased activity in prefrontal areas can increase the cholinergic concentration in other cortical areas such as posterior parietal cortex (Nelson et al., 2005). The parietal cortex is involved in spatial (Posner et al., 1987) and temporal (Koenigs et al, 2009) aspects of attention. These results support the regulative component of posterior parietal cortex and prefrontal areas in attentiondependent ACh release. Especially the prefrontal cortex is thought to perform “top-down" cholinergic regulation (Sarter et al., 2005, Parikh and Sarter, 2008).

The results just mentioned rely on microdialysis, a method used to continuously measure the concentration of endogenous molecules like neurotransmitters in 
the extracellular fluid. The low temporal resolution of ACh microdialysis measures (minutes) presents difficulties in linking cortical cholinergic release to the fast effects of attentional modulation. A final limitation is that most of the related pharmacological research has been performed on rodents, while the main relevant results and models of attention arise from research in non-human primates and humans.

\section{Attentional modulation can be directly linked to acetylcholine function}

Studies performed by Thiele and colleagues directly link attentional modulation and cholinergic function in awake, behaving macaque monkeys. They refined the relatively old method of iontophoresis, producing a novel three-barrel electrode-micropipette. Here, the application of a small current controls the release of a current-dependent amount of charged agonist or antagonist into the tissue surrounding the micropipette in a highly localized manner, while singleunit activity is simultaneously recorded (Thiele et al, 2006). The study was performed to shed light on the underlying cellular mechanisms of the welldiscovered effects of spatial attention on firing rates in the early visual area V1 (Herrero et al., 2008). While the monkey performed a top-down spatial attention task (attending inside the neuron's receptive field or outside of it), Herrero and colleagues performed a block-wise pharmacological manipulation in the direct vicinity of the recorded neuron in area V1 using acetylcholine, scopolamine (a muscarinic antagonist) or mecamylamine (a nicotinic antagonist). They could show that a moderate increase of ACh led to an increase in attentional modulation of firing rates. In addition, they found muscarinic, but surprisingly not nicotinic, receptor contribution to attentional modulation in area $\mathrm{V} 1$ of macaque monkey. 
However, it still remains an unanswered question how the fast and local effects of spatial attention can be associated with the coarse temporal and spatial activation pattern of ACh. One possible explanation is an interaction of the cholinergic system with the glutamatergic and GABAergic system. It is known that glutamatergic as well as GABAergic neurons express muscarinic AChRs (McCormick and Prince, 1985, Disney et al., 2006), and their fast activation can therefore be triggered by ACh. Another possible source for the highly localized action of spatial attention is cholinergic cortical bipolar interneurons (von Engelhardt et al., 2007). These interneurons are predominantly found in layer 2-3 in almost every cortical area (Eckenstein and Thoenen, 1983). They follow a columnar orientation (von Engelhardt et al., 2007) and therefore constitute a locally restricted cholinergic source, influencing only small cortical units.

\section{V1 vs. MT - receptor contribution to attentional modulation}

Based on anatomical studies it is questionable whether the primary visual cortex V1 serves as a suitable model for cholinergic modulation of visual cortical circuits in the macaque, as it has been shown that the percentage and subtype composition of inhibitory neurons in V1 differs from other cortical areas (DeFelipe et al., 1999). There are, however, similarities between V1 and other cortical areas like the extrastriate area MT. Muscarinic receptors of the subtype M1 were found to be equally expressed by inhibitory neurons showing a fastspiking physiological phenotype (Disney et al., 2014) in area V1 and MT. This muscarinic subtype is therefore a possible global candidate to mediate spikerate by attention, assuming the muscarinic type is the main mediator. Although a nicotinic receptor contribution in V1 could not be shown, Disney and colleagues suggest a homomeric nicotinic receptor contribution in extra striate areas, whereas a heteromeric receptor contribution is virtually impossible due to its low occurrence (Disney et al., 2014). 
As mentioned earlier, the magnitude of visual attention effects increases throughout the visual hierarchy. Combining the highly representative cholinergic receptor distribution with the strong attentional effect on motion processing, area MT is a perfect candidate to link attentional modulation with cholinergic contribution and serves as a good model for cholinergic modulation of visual cortical circuits in the macaque. A recent study done in area MT of anesthetized monkeys investigated the effect on neuronal tuning curves by ACh injection, and whether these effects are similar to attentional modulation (Thiele et al., 2012). By showing a cholinergic induced increase in firing rate with unchanged directional tuning, as well as reduced noise correlations, they support the idea that ACh has an important contribution to attentional modulation. However, the study was done in anesthetized monkeys and the anesthesia used is known to interact with the cholinergic system (Thiele et al., 2012). For that reason a study in awake, behaving monkey is crucial to directly link attention-induced changes in neuronal response to cholinergic activity. 


\section{Original manuscripts}

The following chapter contains the following manuscripts:

Human linear visual motion direction discrimination thresholds: Graded deployment of spatial attention shows hemifield dependent resources

Vera Katharina Veith, Stefan Treue; prepared for submission

Author contribution: VKV and ST designed the experiment. VKV, Jan Lause and Vighneshvel Thiruppathi collected the data. VKV analyzed the data. VKV produced the plots and corresponding scripts. VKV wrote the manuscript.

\section{Human linear visual motion direction discrimination thresholds: Effects of graded deployment of spatial attention and signal strength}

Vera Katharina Veith, Stefan Treue; prepared for submission

Author contribution: VKV and ST designed the experiment. VKV, Philipp Ulbrich and Farina Bubert collected the data. VKV analyzed the data. VKV produced the plots and corresponding scripts. VKV wrote the manuscript. 
A pressure injection system for investigating the neuropharmacology of information processing in awake behaving macaque monkey cortex

Vera Katharina Veith, Cliodhna Quigley, Stefan Treue; J. Vis. Exp. (109), e53724, doi: 10.3791/53724 (2016).

Author contribution: VKV implemented the injection technique. VKV and CQ collected the data. VKV and CQ analyzed the data. VKV and CQ produced the plots and corresponding scripts. Johannes Veith made the picture. VKV and CQ wrote the manuscript. ST edited the manuscript. All authors discussed the results and commented on the manuscript.

The role of the cholinergic system in attentional modulation in area MT of the primate visual cortex

Vera Katharina Veith, Cliodhna Quigley, Stefan Treue; prepared for submission

Author contribution: VKV trained one monkey. VKV and CQ trained the second monkey. VKV and CQ collected the data. VKV and CQ analyzed the data. VKV and CQ produced the plots and corresponding scripts. VKV wrote the manuscript. 


\title{
Human linear visual motion direction
}

\section{discrimination thresholds: Graded}

\section{deployment of spatial attention shows}

\section{hemifield dependent resources}

\author{
Vera Katharina Veith ${ }^{1}$, Stefan Treue ${ }^{1,2}$ \\ ${ }^{1}$ Cognitive Neuroscience Laboratory, German Primate Center, Goettingen, Germany \\ ${ }^{2}$ Bernstein Center for Computational Neuroscience, Goettingen, Germany
}

\section{Abstract}

Attending to one location in the visual field, guided by a pre-cue, leads to enhanced processing of the stimuli presented at that location, resulting in better performance and reduced reaction time (Treue \& Maunsell, 1996; Posner, 1980). When the information about the subsequent target location is diminished or not provided at all, a behavioral cost is observed, in terms of a decrement in perceptual performance (Posner, 1980). Several psychophysical studies are not able to distinguish, whether the measured improvement in behavioral performance is ascribed to a reduction in stimulus uncertainty or to an actual improvement in information processing (Pashler, 1994; Pashler and Johnston, 1998). In this study, with the use of a novel task design, we are able to measure the pure influence of varying magnitudes of spatial attention on human 
subjects' performance in discriminating visual motion directions. An endogenous cue was used to gradually guide spatial attention to two stimuli, either placed across the two hemifields or within the left hemifield. Therefore we were additionally able to measure hemifield dependence on spatial attention capacities.

In the classical stimulus arrangement (across hemifield), observers were as good in simultaneously monitoring two spatial locations, than monitoring just one location. Even if deployed spatial attention was further diminished (25\% attentional deployment), subjects' performance was not significantly influenced. In contrast, when spatial attention was distributed within one hemifield, behavioral performance was deteriorated. This suggests that the decrement in performance is due to common processing resources, that the two stimuli are competing for when they are placed within one hemifield (Sereno and Kosslyn, 1991). These attentional capacity limitations seem to underlie anatomical constraints (Alvarez and Cavanaugh, 2005). 


\section{Introduction}

Spatial visual attention is a mechanism that upon deployment improves perceptual performance. Vision at an attended location is faster (e.g. Posner, 1980), more accurate, of higher spatial resolution (Yeshurun and Carrasco, 1998) and enhances sensitivity for fine changes (Carrasco et al, 2004). Not attended stimuli appear to have lower contrast or (if they are objects embedded in complex scenes) might not be perceived at all (Carrasco, 2006, Reynolds \& Chelazzi 2004).

Physiologically, attention has been reported to enhance neuronal responses in visual cortex to stimuli, when they are attended (Treue \& Maunsell, 1996). Such a gain increase can increase neuronal performance and therefore offers a possible mechanism of attentional perceptual enhancement (Cook \& Maunsell, 2002). Nevertheless, while the neuronal response enhancement to visual motion stimuli has been well-investigated and documented in area MT - an extrastriate cortical area thought to underlie the perception of linear motion - it remains controversial if and how much attention enhances the perception of basic visual features, such as visual motion (Liu, Fuller \& Carrasco, 2006; Braun \& Julesz, 1998).

Additionally, it is still under debate how spatial attention is deployed in the visual field. In contrast to early studies showing the existence of only one spotlight of attention eliciting enhanced performance at that location (Posner et al., 1980, Eriksen \& St. James, 1986), an increasing amount of recent studies argue in favor for the existence of (at least) two non-continuous regions in space that can be attended simultaneously (Awh \& Pashler, 2000; Mueller et al., 2003). Moreover, it was reported that information of intervening regions could be ignored (Shaw \& Shaw, 1977; Shaw, 1978; Awh \& Pashler, 2000). Neuronal evidence supports this notion, showing that two spatially separated stimuli elicit 
higher fMRI signal at the two corresponding retinotopic regions (McMains \& Somers, 2004, Morawetz et al., 2007).

As proposed by the hypothesis of the bilateral advantage of attentional distribution, attention can be deployed to two locations across the two hemifields without any cost being involved, whereas splitting attention within one hemifield uncover attentional resource limitations (Reardon et al., 2009; Sereno and Kosslyn, 1991). The inter-hemispheric advantage is ascribed to the anatomical and functional independence of the two cerebral hemispheres, also showing full functionality, when the inter-hemispheric communication is disrupted by a surgical transection of the corpus callosum (Liederman, 1998, Luck et al., 1989). This means, when the two stimuli are presented in an acrosshemifield arrangement, it is suggested that each stimulus is processed independently, whereas when both stimuli are placed within one hemifield, they seem to access the same source. However, visual field representations of extra striate cortical areas for the lower and upper part of the visual field are also often found to be anatomically segregated (Van Essen, 1985).

Here we investigate the effects of spatial attention on performance in a direction-discrimination task, using two moving dot patterns placed either across both hemifields or within the left hemifield. The overall aim of our study is to investigate the effects of various levels of spatial attention onto the two stimuli in dependence of their spatial configuration. Therefore, we aim to extent the bilateral attentional advantage, as previously shown for higher-level tasks (Alvarez and Cavanagh, 2005,) as well as for elementary visual tasks (Reardon et al., 2009), to the linear motion discrimination task.

In many cases, divided attention tasks suffer from larger stimulus uncertainty rather than the corresponding undivided attention (Posner et al., 1980; Pashler, 1994). We controlled for such effects, by using a four alternative forced choice (4AFC) task, allowing us to determine those trials in which performance suffered from errors due to stimulus uncertainty. We manipulated the validity 
of the cue in our paradigm across three values. This allowed us a fine-grained analysis of the effect of graded attention (100-25\%) onto our stimuli. The effect of attention on performance is investigated by comparing subjects' performance when various proportions of attention were allocated to a given stimulus.

With our chosen stimuli, we aim to target area MT, an extrastriate cortical area thought to underlie the perception of linear motion. As area MT is involved in early stages of cortical processing (Vanni et al., 2004), we were targeting bilateral vs. unilateral differences.

We demonstrate, that subjects can attend to two spatial locations across the two hemifields simultaneously without showing any cost in linear movement discrimination when spatial attention is gradually diminished. When stimuli were shown unilateral, splitting attention to two stimuli incorporates a cost and subjects showed a reduction in performance. Based on our results, we can support the notion of two independent attention systems, one being active on each hemifield, as we found bilateral attention advantage for linear motion direction discrimination tasks.

\section{Material and Methods}

\section{Observers}

12 observers (9 females), including the author (VV), accomplished the acrosshemifield task. All observers, except VV, were naïve concerning the purpose of the experiment. All subjects have normal or corrected to normal vision. They gave a written informed consent and were paid for their contribution (except for VV). 


\section{Setup and stimuli}

The visual stimuli were presented in a dark room on a 22" widescreen TFT monitor (Syncmaster 2233RZ, Samsung, Seoul, South Korea), with 1680 x 1050 pixels resolution and a refresh rate of $120 \mathrm{~Hz}$. Viewing distance of the observer to the monitor was kept constant at $57 \mathrm{~cm}$ using a chinrest. A commercial gamepad (Logitech International S.A., Switzerland) was used for answering. Eye position was recorded using an eye tracker (EyeLink, SR research).

The stimuli were generated and presented using MWorks, an open source application, built for real time experiments. Used stimuli were white random dot patterns (RDP) presented on a uniform black background. Each dots' size was $0.0564 \mathrm{deg}$ and they were shown with a density of $10 \mathrm{dots} / \mathrm{deg}^{2}$. Each patch had a diameter of $5 \mathrm{deg}$ moving with a speed of $8 \mathrm{deg} / \mathrm{s}$ within stationary apertures. Always two patches were shown simultaneously, being centered at a constant eccentricity of 5 deg either left or right on horizontal meridian (acrosshemifield experiment) or up or down in the left hemifield (within-hemifield experiment).

In both experimental conditions, a centrally located white square (side length $0.2 \mathrm{deg}$, luminance $72 \mathrm{~cd} / \mathrm{m}^{2}$ ) was used as a fixation point. A red isosceles triangle (side length $0.4 \mathrm{deg}$ ), serving as a endogenous pre-cue, was presented centrally instead of the fixation point, pointing either to the left or the right location (across-hemifield condition) or pointing leftward tilted upwards or downwards (within-hemifield condition). All stimuli were presented on a black background with a luminance of $0.3 \mathrm{~cd} / \mathrm{m}^{2}$.

\section{Task design}

Within this experiment, two spatial configurations of stimuli were tested; either two stimuli were presented across the two hemifields or within the left 
hemifield. The temporal structure as well as the stimuli attributes stayed constant across the two experimental conditions. The detailed trial sequence for both experimental conditions is depicted in Figure 1.

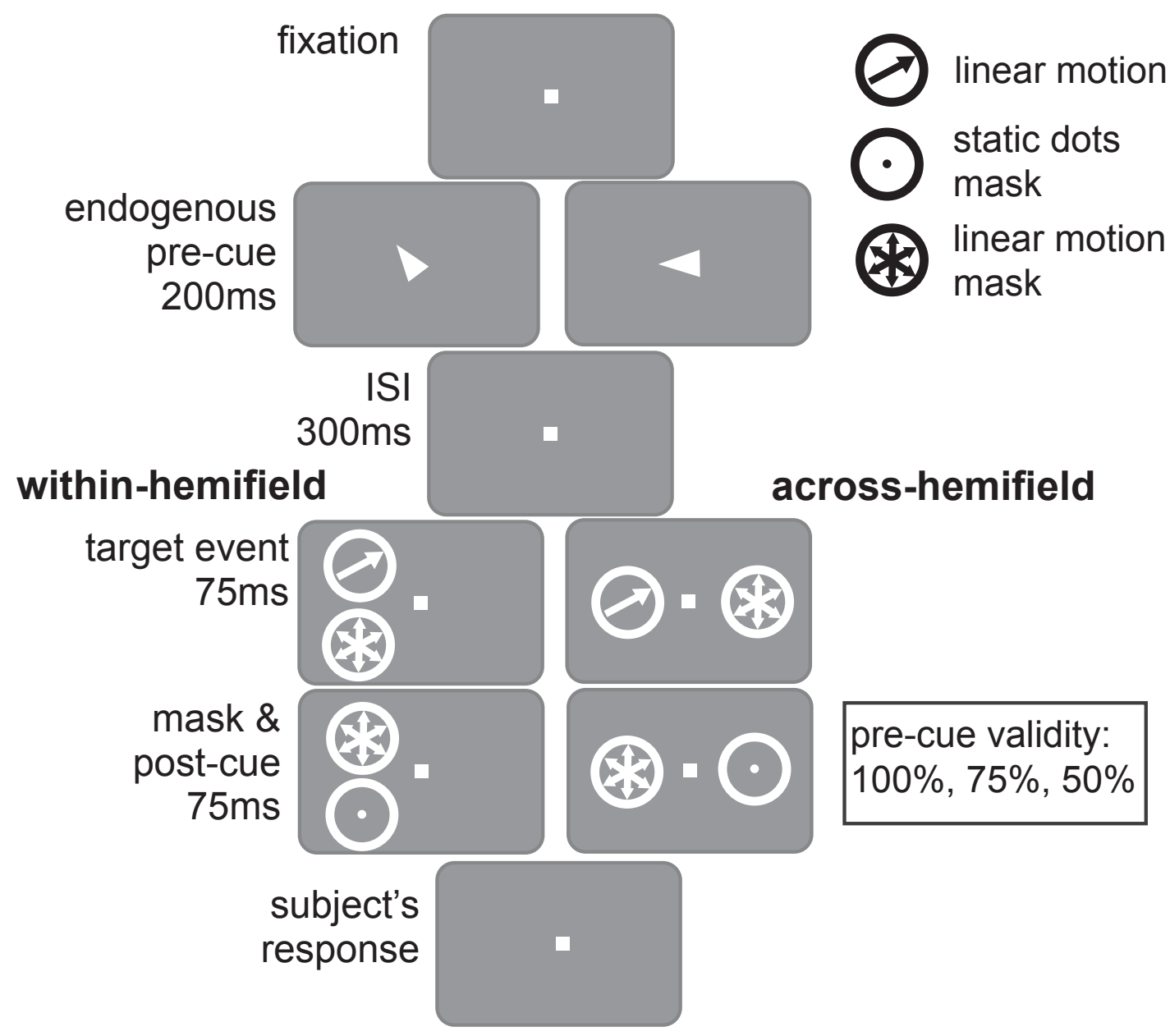

Figure 1. Trial sequence for both experimental tasks. Subjects' spatial attention was guided by an endogenous pre-cue with block wise changing validity. Spatial attention was either deployed across both hemifields (right) or within the left hemifield (left). Subjects were instructed to report the linear motion direction of the target dot pattern (up or down) by pressing one of four buttons on the gamepad (4-alternative forced choice design).

Subjects were asked to maintain fixating the centrally presented fixation point throughout the whole trial, which was controlled by an eyetracker. Each trial sequence was initiated by pressing the start button on the gamepad. 
Immediately after button press the centrally presented fixation point was displaced for $200 \mathrm{~ms}$ by the endogenous pre-cue. After an inter stimulus interval of 300ms, two RDP stimuli were shown either at a left and right position on horizontal meridian with respect to the central fixation point (across-hemifield condition) or up and down within the left hemifield (withinhemifield condition). One RDP contained the task-relevant stimulus (target), a brief coherent linear motion signal (75 ms), moving towards the fixation point. The second RDP contained fully incoherent linear motion (distractor) moving to all possible directions. The target stimulus was immediately followed by a random motion mask for $75 \mathrm{~ms}$ to stop information uptake. At distractor location a static dot pattern was shown for the same duration, acting as a postcue. For the across-hemifield condition, subjects were asked to indicate the location (left or right) and to discriminate the motion direction (up or down relative to horizontal) of the target stimulus by pressing one out of four buttons on a gamepad, e.g. left upper button should be pressed for a linear movement above the horizontal line within the left RDP. Regarding the within-hemifield experiment, subjects were asked to indicate the location (upper left or lower left) by pressing one of two buttons and then to discriminate the motion direction (up or down relative to horizontal) by giving a second answer, pressing one of two buttons. The subjects were asked to report as accurate as possible the perceived direction of the target RDP without receiving any feedback about their decision.

Endogenous attention was manipulated for both experimental conditions by presenting three different levels of pre-cue validity (100\%, 75\% and 50\%) in a block wise manner. Each block consisted of 320 trials. The $75 \%$ cue validity condition was performed twice (640 trials in total), in order to achieve an appropriate amount of repetitions for the invalid trials. Observers were informed about the depict pre-cue validity condition in advance of every block. 


\section{Training of observers}

In order to avoid learning effects in recording sessions, subjects were trained intensively within three training sessions ( $1.5 \mathrm{~h}$ duration) performed on three consecutive days. In total, subjects performed all possible condition combinations with at least 3840 trials within the whole training procedure. After training session, a minimum criterion of performance (4 deg discrimination threshold in $100 \%$ cue validity condition) had to be reached in order to be included in the following recording sessions. Final data recording was performed on two or three consecutive days for the two experimental conditions. Across experimental sessions the order of experimental blocks was randomized.

\section{Staircase procedure}

For both experimental conditions the shown linear motion direction in the target dot pattern was varied dynamically using interleaved staircases achieving a rational amount of repetitions close to the point of subjective equality (PSE).

In total, four randomly interleaved staircases were used per RDP, starting at a fixed levels of 5 deg above and below of horizontal (o deg), changing with a factor of 1.25. In detail, two staircases were active on one RDP when a valid precue was shown, whereas two independent staircases acted when there was an invalid trial. All staircases followed a 2 to 1 rule, converging when observers' answer was twice consecutive consistent with shown direction and diverging when the answer was inconsistent. 


\section{Data analysis}

The task was designed to gradually manipulate the distribution of spatial attention either across both hemifields or within one hemifield. By using block wise different cue validity the subjects were instructed to change behavior accordingly.
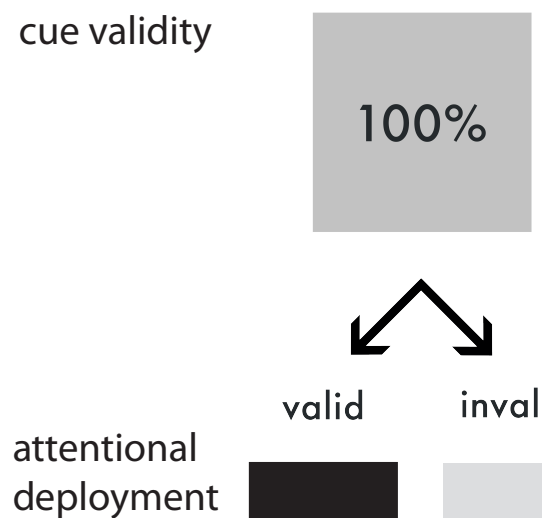

deployment

valid
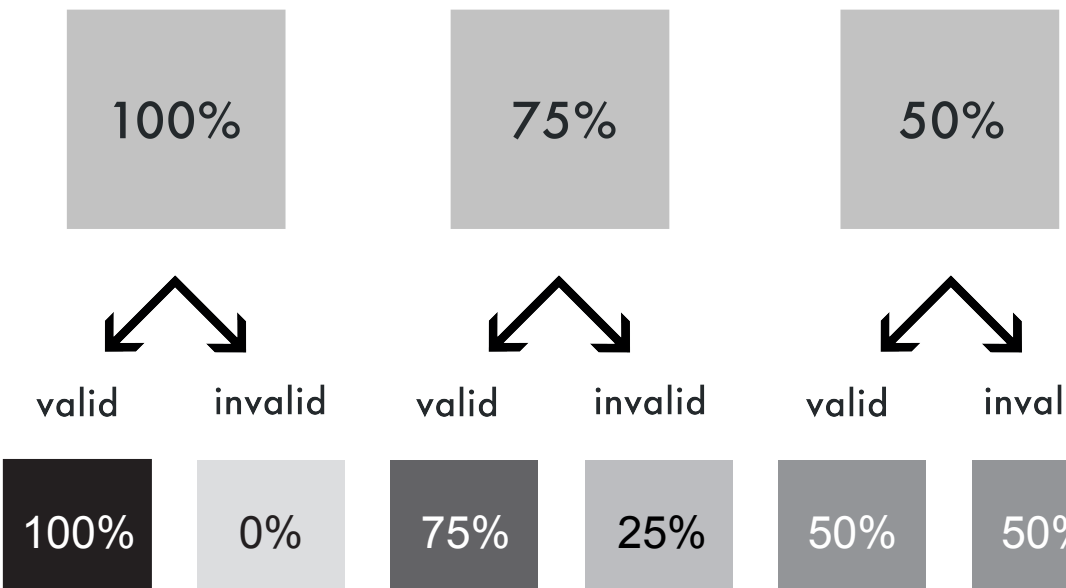

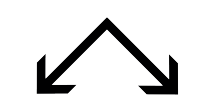

valid

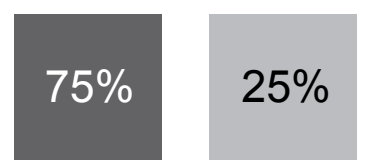

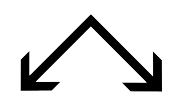

valid

invalid

$50 \%$

Figure 2. Three levels of pre-cue validity, shown in a block wise manner, were used in this study. They led to four levels of attentional deployment (100\%, 75\%, 50\%, and 25\%).

Depending on cue validity the level of attention was calculated (see Figure 2). For example for $75 \%$ cue validity, subjects were instructed to gradually deploy their attention to the two spatial locations, attending the cued locations proportionally more than the uncued location. Therefore the cued location was seen as $75 \%$ attended and the uncued location as $25 \%$ attended.

With this 4AFC task design we were able to distinguish between two different error types. Only trials where the observer indicated the correct stimulus position (e.g. pressing one of the two left buttons for the target stimulus being left) where included in further analysis, whereas responses to the distractor 
were seen as location errors and rejected. Hence, with this task design we are able to eliminate the spatial uncertainty as a critical factor from our results. Therefore, we are able to measure changes in performances solely based on attentional deployment.

Psychometric functions were fitted for data collected at both target dot pattern locations and for valid and invalid trials separately using a maximum likelihood procedure combined with bootstrap sampling (Palamedes Toolbox, Kingdom \& Prins). A cumulative normal function was used to model psychometric data.

The Cumulative Normal distribution is given as:

$F_{N}(x ; \alpha, \beta)=\frac{\beta}{\sqrt{2 \pi}} \int_{-\infty}^{x} \exp \left[-\frac{\beta^{2}(x-\alpha)^{2}}{2}\right]$,

where parameter $x$ is the signal strength, $\alpha$ corresponds to the threshold and $\beta$ corresponds to the reciprocal of the standard deviation of the normal distribution and determines the slope.

The discrimination threshold, here defined as the first deviation of the fitted psychometric function, was estimated for the two spatial locations, for every experimental condition, as well as for cue validity separately and served as a measure of the change in performance. The discrimination thresholds calculated were merged for the left and right stimulus position (acrosshemifield) or for the upper and lower stimulus (within-hemifield) for every attentional condition separately by calculating the weighted mean. The inverse of the $95 \%$ confidence interval served as a weight factor.

Across subjects, the weighted arithmetic mean was again calculated for the corresponding experimental condition. The weighted direction discrimination thresholds were compared with a repeated-measures ANOVA using Statistica software (Statistica 12, StatSoft). Effects of different attentional levels and different stimulus positions were compared with a $4 \times 2$ repeated measures 
ANOVA with the factors attention and stimulus position. Post-hoc comparison was performed using Fisher's Least Significant Difference.

\section{Data simulation}

In order to confirm the involvement of spatial attention for task solving, the discrimination thresholds for the four different attentional levels were simulated and compared to mean discrimination thresholds for the recorded data. Simulated data was generated assuming that the subjects were not able guide the spatial attention based on the pre-cue and therefore treated the cue always as $100 \%$ valid, independent on task instruction. Performance at the uncued location was therefore calculated based on answers given in a random fashion. For example, when one of the two locations is randomly cued (50\% attention condition), in one half of the trials the target stimulus is cued and the performance is therefore similar to the $100 \%$ attention condition. In the other half of the trials, the target stimulus is untracked and therefore answered in a random fashion.

\section{Results}

The aim of this experiment was to gradually manipulate the deployment of spatial attention to two locations in the visual field. The two locations were either placed across both hemifields or within the left hemifield.

Thus, we compared direction discrimination thresholds for three different cue validity conditions that guide attentional deployment to investigate the impact of spatial attention on performance. In a first step we investigated the gradual deployment of attention for the classical stimulus arrangement (across- 
hemifield). Figure 3 depicts the effect of cue validity on performance for a sample subject for the left random dot pattern in an across-hemifield stimulus placement.

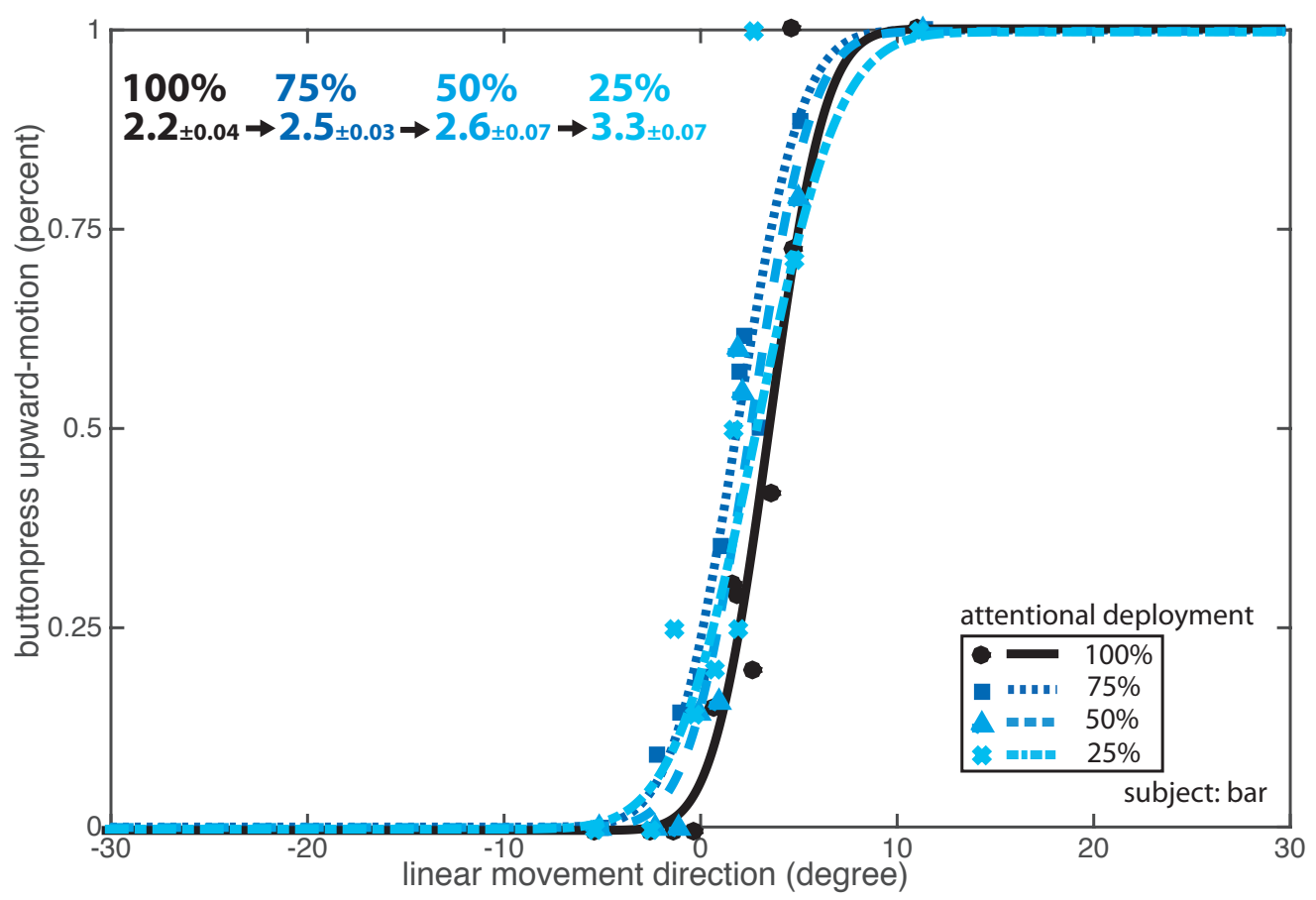

Figure 3. Behavioral results of a sample subject. The percentage of upward-button press is plotted for every shown linear movement direction of the stimulus. Data was fitted using cumulative normal distribution for all attentional levels separately. The direction discrimination threshold was defined as the first deviation of the fitted psychometric function. The small numbers indicate the $95 \%$ confidence interval.

For this sample subject, a stepwise reduction in attentional deployment guided by the cue validity led to a stepwise increase in direction discrimination threshold diagramed by the steepness of the psychometric functions. The effects for $75 \%$ and $50 \%$ of attentional deployment ( $2.5 \pm 0.03$ and $2.6 \pm 0.07 \mathrm{deg})$ led to a mild increase in direction discrimination compared to the full-attended condition $(2.2 \pm 0.04) .25 \%$ of attentional deployment showed strongest impact on direction discrimination $(3.3 \pm 0.07 \mathrm{deg})$. 
In total, 12 subjects were included in final data analysis as they fulfilled the initial inclusion criterion.

Repeated measures ANOVA with main factors attention and stimulus position revealed significant main effect of attention $(\mathrm{F}=4.53, \mathrm{p}<0.01)$ and stimulus position $(\mathrm{F}=11.11, \mathrm{p}<0.001)$ on direction discrimination, whereas the interaction of both factors did not show significance $(F=2.16, p=0.1)$. This data suggests that the direction discrimination thresholds vary dependently as a function of proportion of attention and stimulus position.

Figure 4 depicts the weighted mean direction discrimination thresholds across all subjects according to attentional allocation for the classical stimulus arrangement (across-hemifield) and for the within-hemifield stimulus arrangement. On average, subjects were able to discriminate a direction change of 2.65 \pm 0.23 (Standard error of mean, SEM) degrees when fully attending one spatial location, when objects were distributed across both hemifields. 


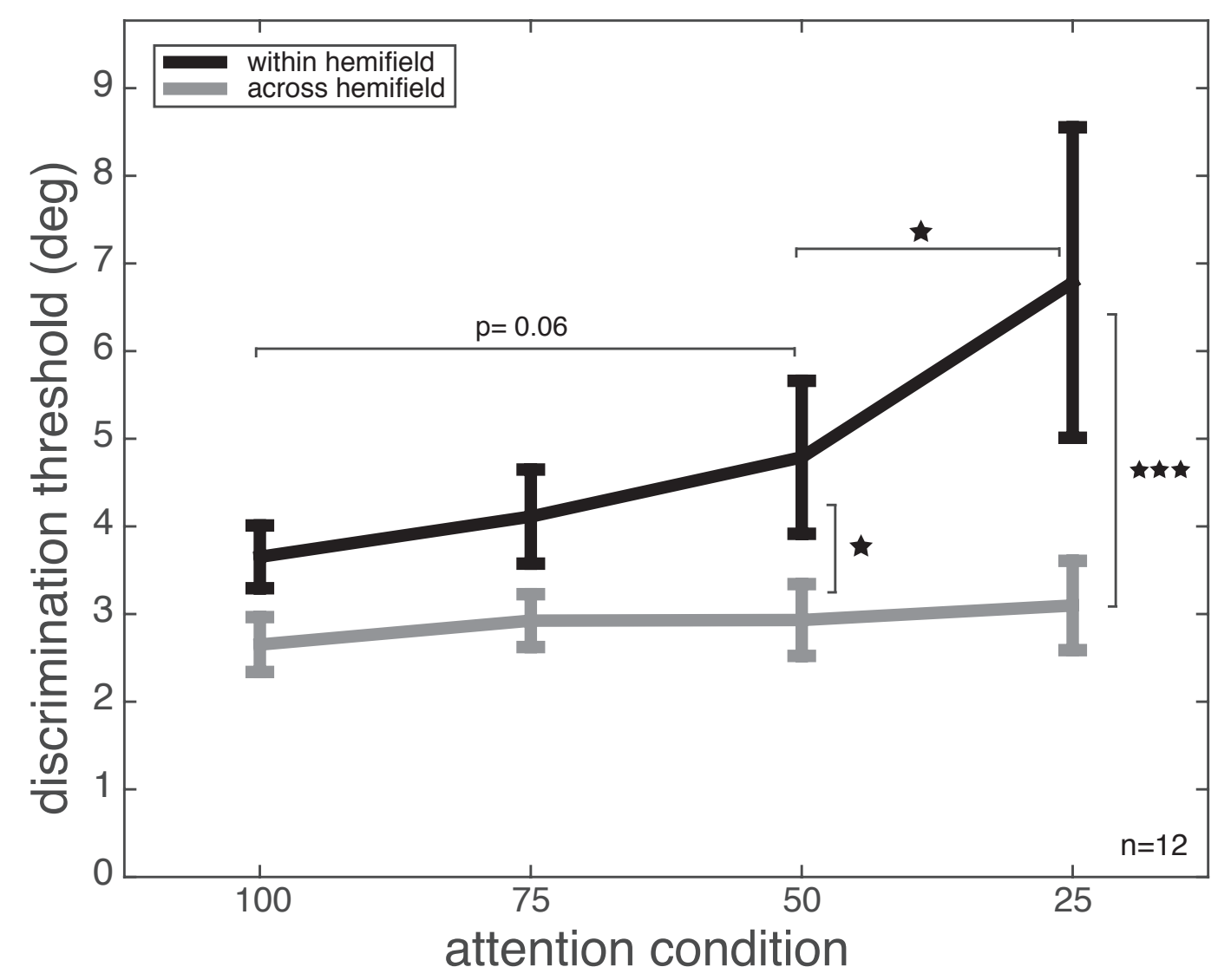

Figure 4. Weighted mean direction discrimination threshold. For each attentional condition the averaged discrimination threshold was plotted across 12 subjects. Stimuli were either placed across both hemifields (grey) or within one hemifield (black). Error bars indicate the standard error of mean.

The weighted mean across subjects increased marginally with decreasing amount of deployed spatial attention, 2.92 $\pm 0.23,2.93 \pm 0.29$ and $3.1 \pm 0.43$ for $75 \%, 50 \%$ and $25 \%$ attentional condition respectively, when both stimuli are displayed across both hemifields. However, no significant change was observed using repeated measure with Fischer LSD post-hoc test (100vs75: $F=4.86$, $\mathrm{p}=0.87$; 100vs50: $\mathrm{F}=4.86, \mathrm{p}=0.72 ;$ 100vs25: $\mathrm{F}=4.86, \mathrm{p}=0.44 ; 75 \mathrm{vs50}: \mathrm{F}=5.06$, $\mathrm{p}=0.84 ; 75 \mathrm{vs} 25: \mathrm{F}=5.06, \mathrm{p}=0.55 ;$ Oovs25: $\mathrm{F}=5.31, \mathrm{p}=0.69$ ). 
Secondly we were interested, whether stimuli placement within one hemifield shows impact on direction discrimination. Again, we compared the direction discrimination thresholds for the different values of attentional deployment. When deploying full attention to one stimulus within the left hemifield, performance was not significantly different to $100 \%$ attentional deployment in the across-hemifield stimulus configuration, showing an average discrimination threshold of $3.7 \pm 0.36$ degree $(F=4.45, p=0.64)$. Even if deployed attention was further diminished to a level of $75 \%$, the averaged discrimination threshold of $4.1 \pm 0.54$ did not show significant difference to across-hemifield stimulus configuration ( $\mathrm{p}=0.56, \mathrm{~F}=6.26$ ). Remarkably, when subjects were instructed to simultaneously attend two spatial locations within the left hemifield, a strong decline was observed compared to full attentional deployment. Here subjects were able to discriminate a linear movement when an angle of $4.8 \pm 0.87$ degrees was shown. This decrease in performance shows trend towards significance compared to $100 \%$ attentional deployment $(F=6.26, p=0.06)$. This is equivalent to a decline in performance of $27 \%$, compared to full spatial attention. With a further reduction of spatial attention within one hemifield, performance was further reduced. Here the discrimination threshold was on average $6.8 \pm 1.8 \mathrm{deg}$, equivalent to an increase of more then $84 \%$ compared to full attention condition discrimination threshold. This value is significantly different to value with $100 \%$ of attentional deployment $(\mathrm{F}=10.1, \mathrm{p}<0.001)$. Also compared to the other two levels of attentional deployment, the difference is significant (25vs50: $\mathrm{F}=10.1, \mathrm{p}=0.49 ;$ 25vs75: $\mathrm{F}=10.1, \mathrm{p}<0.001$ ).

With the 4AFC task design used, we were able to distinguish between two different error types; answering at distractor location (location error) vs. answering at target location but indicating wrong linear movement direction. Only trials where the observer indicated the target stimulus position (e.g. pressing one of the two left buttons for the target stimulus being left) were included in further analysis, whereas responses to the distractor were seen as location errors and were therefore rejected. 


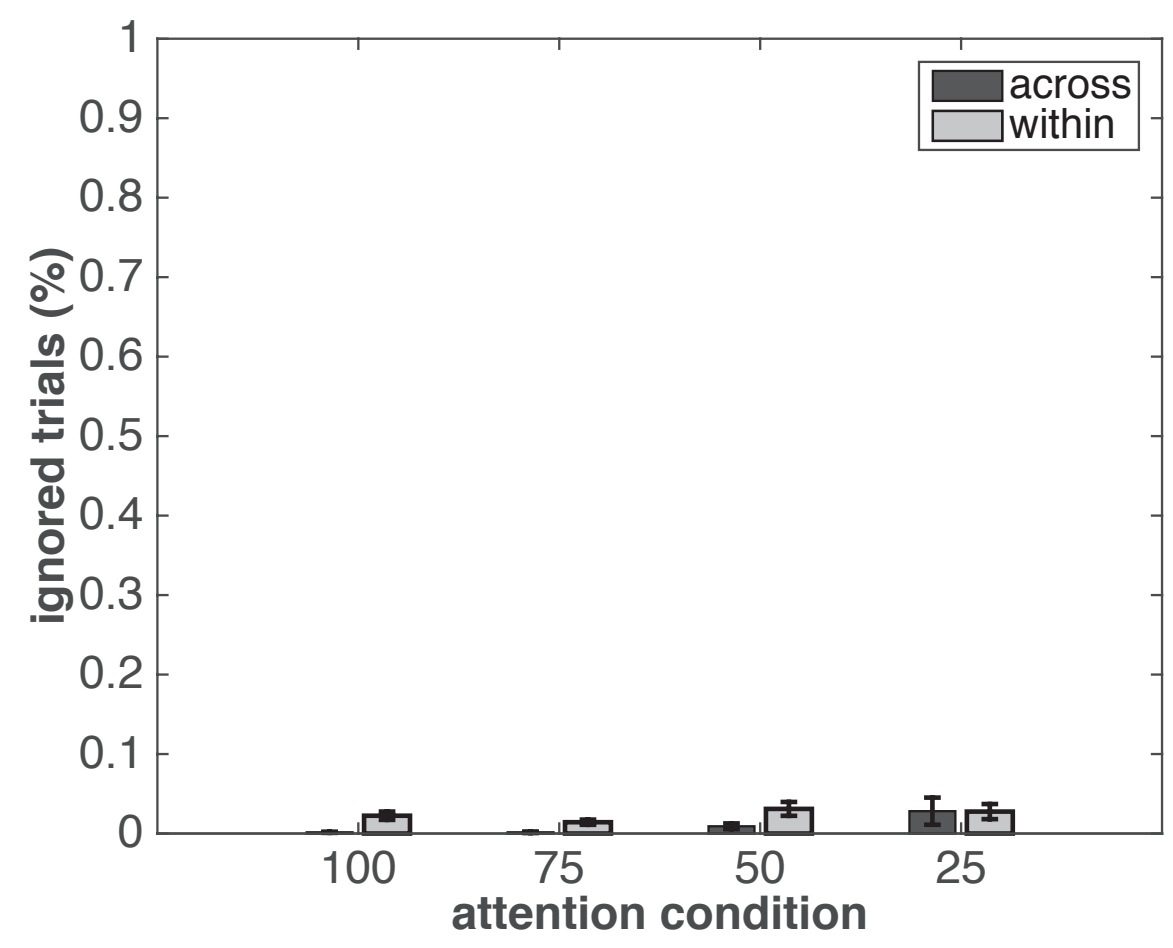

Figure 5. Averaged percentage of ignored trials across 12 subjects. Trials were considered being ignored, when subjects answered at distractor location. Error bars indicate the standard error of the mean.

Figure 5 depicts the averaged percentage of ignored trials among subjects for all attentional conditions and spatial configurations separately. Overall, the percentage of ignored trials is extremely low; being always below $1 \%$ of all performed trials and can be therefore neglected.

As the task used in this study requires voluntary deployment of spatial attention, it is mandatory to confirm that subjects follow task instructions and adjust their behavior accordingly. In order to control for comprehension of the endogenous pre-cue and to confirm the involvement of spatial attention for task solving, subjects behavior was simulated to the effect that the cue was always treated as $100 \%$ valid, independent of task instruction. In valid trials the subject would therefore answer with its known performance, whereas in invalid trials, the subject would answer on chance level. This simulation consequently would 
assume that a simultaneous deployment of attention to two spatial locations would not be possible.

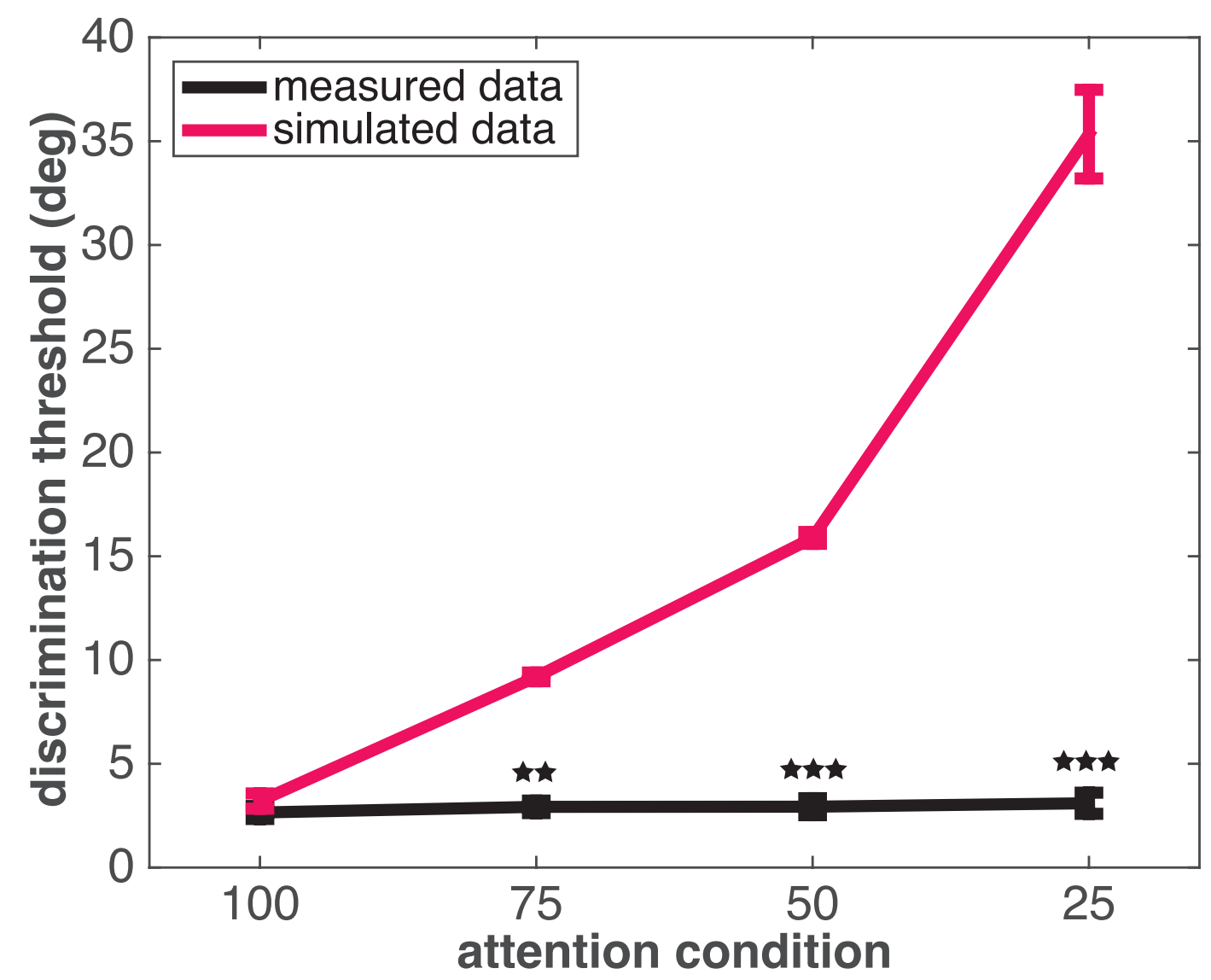

Figure 6. Comparison of simulated data and measured data (across-hemifield stimulus arrangement). Subject's behavior was simulated (pink) to the assumption, that two spatial locations cannot be attended simultaneously. Invalid trials are therefore simulated to be answered at chance level. For a detailed description of the simulation see text. Errors indicate standard error of the mean.

As shown in Figure 6 this strategy would lead to a significant increase in discrimination threshold for all attentional conditions (post-hoc comparison simulated vs. measured data; $75 \%$ attention: $\mathrm{F}=4.92, \mathrm{p}=0.004 ; 50 \%$ attention: $\mathrm{F}=5.1, \mathrm{p}<0.001 ; 25 \%$ attention: $\mathrm{F}=5.34, \mathrm{p}<0.001)$. Simulated discrimination thresholds were 3 -fold higher than the measured data for the $50 \%$ attentional deployment or even 10 -fold higher for $25 \%$ of attentional deployment. 


\section{Discussion}

With our paradigm we aim to gradually manipulate spatial attention to two spatial locations, either placed across both hemifields or within one hemifield. The task design used, allowed to measure the pure attention effects on to the two stimuli. The effect of graded attention on performance was measured, calculating the direction discrimination threshold for linear motion for every spatial stimulus configuration separately. We found bilateral attention advantage for linear motion direction discrimination. Simultaneous attendance of two stimuli, placed across the two hemifields, did not seem to involve any attentional cost, whereas splitting attention within one hemifield led to a deterioration in performance for linear movement judgment.

In many cases, divided attention tasks suffer from larger stimulus uncertainty than the corresponding undivided attention task. We controlled for such effects by using a 4AFC task, allowing us to distinguish between two types of errors (wrong location vs. wrong linear movement judgment) and therefore to measure the pure attention effect. Additionally, most task designs, investigating divided attention, are planned as dual-tasks, requiring two consecutive answers (Pashler, 1994). Those task designs suffer from the dual-task interference also known as psychological refractory period, showing a decrement in reaction time or performance for the second answer, when the two stimuli are presented simultaneously or with a short stimulus onset asynchrony (Pashler, 1994, Pashler and Johnston, 1998). We further reduced stimulus uncertainty by presenting a post-cue, indicating the non-target location. The subsequent information about the non-target location does not interfere with the linear movement judgment, as this information is only previously available.

We carefully selected a target stimulus duration, which makes the task perceptually demanding. We therefore ensure a severe decimation in 
performance when only half of the duration is provided, as it would be the case in the splitted attention condition. Furthermore, we perceptually masked the target stimulus to ensure the information uptake to be equivalent to target presentation. Due to the short stimulus presentation time of $75 \mathrm{~ms}$, attentional shifting across the two stimuli can be excluded, as prior studies have discovered a minimum time of several hundred milliseconds to switch spatial attention locations (Reeves and Sperling, 1986, Duncan et al., 1994).

In the classical stimulus arrangement (across-hemifield), observers were as good in simultaneously monitoring two spatial locations than monitoring just one location. This indicates that dividing attention to two spatial locations, which are placed across the two visual hemifields, can be executed without any measurable cost in our task. A possible explanation could be, that the attentional capacity is not at its limits, especially as it could be shown that motion detection has a low attentional demand (Braun and Julesz, 1998). It is assumed, that there is some amount of visual awareness outside the attentional spotlight, permitting the subject to perform at both locations, inside and outside of the spotlight, with equal performance for stimuli with low attentional cost (Braun and Julesz, 1998). In addition to a comparison between full attention and split attention condition, we manipulated the validity of the cue in our paradigm across three values, allowing us a fine-grained analysis of the attentional effect $(100,75 \%, 50 \%, 25 \%)$ onto our stimuli. In our acrosshemifield stimulus arrangement, we couldn't observe any significant stepwise decrement in performance. Even for the lowest level of attentional deployment (25\%), no significant difference was observed compared to full attentional deployment. This result led assume, that a judgment of linear movement direction only requires a very low amount of attentional deployment or is even feasible in absence of attention.

However, this assumption does not hold true, when stimuli are placed within one hemifield. Here, a simultaneous attendance of two stimuli led to a strong 
decrease in performance. In this stimulus arrangement, a further reduction of deployed spatial attention (25\%), led to a further decrease in performance, showing significant differences to all other values of attentional deployment. Again, as we used a very short presentation time, we can exclude a serial processing of the two stimuli. Therefore we can conclude, that attentional splitting is also possible within one hemifield, but it shows major costs even for a task, that was attributed with low attentional demand (Braun and Julesz, 1998).

An additional explanation is the existence of two independent resources of attention, one being active in one hemifield. Therefore no decrease in performance was observed, when just one stimulus was placed in one hemifield. Other behavioral studies confirm this notion, showing that twice as many targets could be tracked when they are places across two hemifields (Alvarez and Cavanagh, 2005, Malinowski et al., 2007, Chakravarthi and Cavanagh, 2009) or showing no impairment in performance when subjects had to identify digits in sequences of digits and letters either on one location or on two locations that are bilaterally arranged (McMains and Somers, 2004). With the results gained in this study, we can confirm a bilateral distribution advantage for linear motion stimuli that was already demonstrated for other stimuli used in spatial attention tasks (Sereno and Kosslyn, 1991, Maertens and Pollmann, 2005, Pollmann, Zaidel, von Cramon, 2003, Reardon et al., 2013).

Physiologically, cells having receptive fields in the same hemifield, are not as clearly anatomically segregated as compared to cells with receptive fields in the opposite hemifield. However, it is known for extrastriate cortical areas, like area MT, the area we aim to target with our stimuli, that there is still a distinction across cells regarding their visual field representation, even if their receptive fields share the same hemifield (Van Essen, 1985). Magnetic resonance imaging confirms this notion, by showing two separate spots of higher activity within one cortical hemisphere, when two spatially distinct locations are attended 
within the corresponding hemifield (McMains and Somers, 2004). Although we are therefore very likely triggering two different neuronal populations with our two stimuli displayed in one hemifield, attentional enhancement of those groups seem to underlie one central source.

Additionally, we applied a simple model simulating performance, when only one stimulus can be attended at one time. We therefore simulated subject's behavior for all attentional conditions in that sense, that performance at the cued spatial location is equivalent to the $100 \%$ attention condition, whereas at uncued locations performance is at chance level. Simulated data exceeded measured data for all attentional levels in the classical stimulus placement. The data simulation indicates, that more than one spatial location must be attended simultaneously, in order to achieve the performance measured in our data.

\section{Conclusion}

In the present experiment, splitting spatial attention across both hemifields did not incorporate any cost, whereas splitting attention within one hemifield showed a decrement in performance. Gradual diminution of spatial attention had no effect on performance for linear motion direction discrimination for all levels of spatial attention $(100 \%, 75 \%, 50 \%, 25 \%)$, when stimuli were placed in a classic task design (across-hemifield). In contrast, when stimuli are located within one hemifield, lower level of attentional deployment (50\% and 25\%) showed a gradual influence on performance. This suggests that the decrement in performance is due to common processing resources that the two stimuli are competing for, when placed within one hemifield (Sereno and Kosslyn, 1991). These results extent the bilateral attentional advantage, as previously shown for higher-level tasks (Alvarez and Cavanagh, 2005,) as well as for elementary visual tasks (Reardon et al., 2009), to the linear motion discrimination task. 


\section{References}

Alvarez GA, Cavanagh P (2005) Independent resources for attentional tracking in the left and right visual hemifields. Psychol Sci. 16(8):637-43.

Alvarez GA, Gill J, Cavanagh P (2012) Anatomical constraints on attention: hemifield independence is a signature of multifocal spatial selection. $J$ Vis. 12(5):9.

Awh E, Pashler H (2000) Evidence for split attentional foci. Journal of experimental psychology: Human perception and performance. 26(2): 834-846.

Braun J, Julesz B (1998) Withdrawing attention at little or no cost: detection and discrimination tasks. Perception \& psychophysics 6o:1-23.

Broadbent DA (1971) Decision and stress. London: Academic Press.

Carrasco M, Talgar CP, Cameron EL (2001) Characterizing visual performance fields: effects of transient covert attention, spatial frequency, eccentricity, task and set size. Spat. Vis. 15:61-75.

Carrasco M, Ling S, Read S (2004) Attention alters appearance. Nat Neurosci. $7(3): 308-13$.

Carrasco, M (2006) Covert attention increases contrast sensitivity: Psychophysical, neurophysiological, and neuroimaging studies. In S. Martinez-Conde, S. L. Macknik, L. M. Martinez, J. M. Alonso, \& P. U. Tse (Eds.), Visual perception. Part I. Fundamentals of vision: Low and midlevel processes in perception - Progress in Brain Research. Amsterdam: Elsevier. pp. 33-70.

Chakravarthi R, Cavanagh P (2009) Bilateral field advantage in visual crowding. Vision Research 49(13): 1638-1646.

Cook EP, Maunsell JH (2002) Attentional Modulation of Behavioral Performance and Neuronal Responses in Middle Temporal and Ventral 
Intraparietal Areas of Macaque Monkey. The Journal of Neuroscience 22(5):1994-2004.

Duncan J, Ward R, Shapiro K (1994) Direct measurement of attentional dwell time in human vision. Nature 369(6478):313-5.

Giordano AM, McElree B, Carrasco M (2009) On the automaticity and flexibility of covert attention: a speed-accuracy trade-off analysis. J Vis. 9(3):30.

Kahneman D (1973) Attention and effort. Englewood Cliffs, NJ: Prentice-Hall.

Kinchla RA (1980) The measurement of attention. In: Nickerson, RS., editor. Attention and performance IX. Erlbaum; Hillsdale, NJ. Psychology Press; pp. 213-238.

Liederman J (1998) The dynamics of interhemispheric collaboration and hemispheric control. Brain Cogn. 36(2):193-208.

Luck SJ, Hillyard SA, Mangun GR, Gazzaniga MS (1989) Independent hemispheric attentional systems mediate visual search in split-brain patients. Nature 342: 543-545.

Liu T, Fuller S, Carrasco M (2006) Attention alters the appearance of motion coherence. Psychonomic Bulletin \& Review 13(6):1091-1096.

Malinowski P, Fuchs S, Müller MM (2007) Sustained division of spatial attention to multiple locations within one hemifield. Neuroscience Letters 414 (1):65-70.

McMains SA, Somers DC (2004) Multiple spotlights of attentional selection in human visual cortex. Neuron 42(4):677-86.

Maertens M, Pollmann S (2005) Interhemispheric resource sharing: decreasing benefits with increasing processing efficiency. Brain Cogn. 58:183-192.

Morawetz C, Holz P, Baudewig J, Treue S, Dechent P (2007) Split of attentional resources in human visual cortex. Vis Neurosci. 24(6):817-26. 
Müller MM, Malinowski P, Gruber T, Hillyard SA (2003) Sustained division of the attentional spotlight. Nature 424(6946), 309-312.

Niebergall R, Khayat PS, Treue S, Martinez-Trujillo JC (2011). Multifocal attention filters targets from distracters within and beyond primate MT neurons' receptive field boundaries. Neuron 72(6), 1067-1079.

Pashler H (1994) Dual-task interference in simple tasks: data and theory. Psychol Bull. 116(2):220-44.

Pashler H, Johnston JC (1998) Attentional limitations in dual-task performance. In: Pashler H, editor. Attention. Hove (United Kingdom): Psychology Press. pp. 155-189.

Pollmann S, Zaidel E, von Cramon DY (2003) The neural basis of the bilateral distribution advantage. Exp. Brain Res. 153:322-333.

Posner MI (1980) Orienting of attention. The Quarterly journal of experimental psychology 32(1):3-25.

Prins N, Kingdom FAA (2009) Palamedes: Matlab routines for analyzing psychophysical data. http://www.palamedestoolbox.org

Reardon KM , Kelly JG, Matthews N (2009) Bilateral attentional advantage on elementary visual tasks. Vision Research 49(7): 691-701.

Reynolds JH, Chelazzi L (2004) Attentional modulation of visual processing. Annual Review of Neuroscience 27:611-647.

Reeves A, Sperling G (1986) Attention gating in short-term visual memory. Psychol Rev. 93(2):180-206.

Sereno AB, Kosslyn SM (1991) Discrimination within and between hemifields: a new constraint on theories of attention. Neuropsychologia 29:659-675.

Shaw ML (1978) A capacity allocation model for reaction time. J. Exp. Psychol. Hum. Percept. Perform. 4:568-598. 
Shaw ML, Shaw P (1977) Optimal allocation of cognitive resources to spatial locations. J. Exp. Psychol. Hum. Percept. Perform. 3:201-211.

Treue S, Maunsell JH (1996) Attentional modulation of visual motion processing in cortical areas MT and MST. Nature 382(6591):539-41.

Van Essen D (1985) Functional organization of primate visual cortex. In Cerebra/ Cortex, Vol. 3, A. Peters and E. G. Jones (Editors), Plenum Press, New York. pp. 259-329.

Vanni S, Dojat M, Warnking J, Delon-Martin C, Segebarth C, Bullier J (2004) Timing of interactions across the visual field in the human cortex. Neuroimage 21(3):818-28.

Yeshurun Y, Carrasco M (1998) Attention improves or impairs visual performance by enhancing spatial resolution. Nature 396:72-75. 


\section{Human linear visual motion direction}

\section{discrimination thresholds: Effects of}

\section{graded deployment of spatial attention}

\section{and signal strength}

Vera Katharina Veith ${ }^{1}$, Stefan Treue Tr,2 $^{1,2}$

${ }^{1}$ Cognitive Neuroscience Laboratory, German Primate Center, Goettingen, Germany

${ }^{2}$ Bernstein Center for Computational Neuroscience, Goettingen, Germany

\section{Abstract}

Simultaneously monitoring of two spatially segregated objects was shown to be challenging compared to monitoring a single object and therefore involves a cost (Bonnel \& Miller, 1994; Bonnel \& Prinzmetal, 1998; Duncan, 1984). Such cost is thought to be a consequence of limited attentional resources (Kahneman, 1973; Broadbent, 1971). In this study we gradually guided visual attention towards two spatial locations, distributed across both hemifields, using endogenous pre-cues of variable validity. Additionally, we manipulated the stimulus' signal strength by varying the percentage of linearly moving dots in the target dot pattern. This allowed for a direct comparison of the influence of attention and signal strength on perceptual performance. As expected, signal 
strength had a significant impact on subjects' performance, with performance improving with increasing signal strength. On the other hand, subjects' performance was not significantly influenced by altering cue validity (attentional influence). The two variables, cue validity and signal strength, both seem to have an independent influence on observers' performance, with no interaction found.

\section{Introduction}

Simultaneous judgment of certain features of two objects has been shown to be challenging compared to the judgment of a single object (Bonnel \& Miller, 1994; Bonnel \& Prinzmetal, 1998; Duncan, 1984). As a result a cost is observed, in the form of a decrement in perceptual performance. This phenomenon is often attributed to the limited capacity of spatial attention (Kahneman, 1973; Broadbent, 1971). Information about the behavioral relevance of a forthcoming spatial location is known to drive attentional mechanisms, which in turn lead to an improvement in behavioral performance (Carrasco, 2011; Pashler, 1998) as well as to a perceptual enhancement (Britten, Shadlen, Newsome \& Movshon, 1992; Carrasco, 2011; Liu, Fuller, \& Carrasco, 2006). However, the magnitude of this attentional driven enhancement is still unclear. Moreover, it seems that different feature dimensions claim different levels of attentional demands, as it was suggested, for example, that letter discrimination requires an higher degree of attentional focus, compared to objects' orientation discrimination (Braun, 1994; Braun, 1998). Here we investigate the effects of spatial attention on perceptual performance in a direction-discrimination task using moving random dot patterns (RDP). We chose linear motion stimuli for our study, as most of our visual information contains motion and is therefore of high relevance. Additionally, with our chosen stimuli we aim to target area MT, an 
electrophysiologically well-described visual area, showing to be strongly influenced by attention (Treue \& Maunsell, 1996). Other studies showed the causality between attentional deployment and linear motion discrimination (Bosworth, Petrich \& Dobkins, 2012; Liu, Fuller \& Carrasco, 2006; Verghese, Anderson \& Vidyasagar, 2013). However, theses studies only cover two states of attention, full and poor attention.

In this study we used pre-cues of different validity in order to achieve a finegrained deployment of spatial attention on to two spatial locations, placed across both hemifields, resulting in four levels of attentional deployment (100\%, $75 \%, 50 \%, 25 \%)$. The general task design chosen, is identical to the one used in the former psychophysical study. The main advantage of this task design is that errors caused by stimulus uncertainty can be eliminated and we are therefore able to measure pure attentional effects on performance. Assuming the limited capacity of attention in parallel processing of visual stimuli and assuming a correct incorporation of the spatial information provided by the cue, a gradual improvement in performance with increasing cue validity is expected.

Additionally, signal enhancement hypothesis reveals that attention leads to an enhancement in stimulus strength (e.g. Posner, 1980; Luck, Hillyard, Mouloua, Woldorff, Clark \& Hawkins, 1996) such as an improvement in spatial resolution (Yeshurun and Carrasco, 1998). We manipulated the signal strength of the target dot pattern by varying the percentage of linearly moving dots in the target dot pattern. This allowed us to equate the effects of attention with the effects of stimulus coherence and to measure their individual as well as their interactive influence on perceptual performance. A gradual reduction in signal strength is also expected to progressively diminish subjects' performance as the inherent information is successively reduced and the linear motion is therefore less salient. We concluded if spatial attention increases the apparent motion coherence, it is expected to induce an improvement in performance especially for weak motion signals. 


\section{Material and Methods}

\section{Subjects}

16 subjects (9 females), including the first author (VV), with normal or corrected to normal vision, participated in this study. All subjects gave written informed consent. All subjects, except for the author, were naïve to the purpose of this experiment and were paid for their participation.

\section{Visual Stimuli}

All stimuli were generated and presented using MWorks, an open source application, built for real time experiments. The stimuli were presented in a dark room on a 22" widescreen TFT monitor (Syncmaster 2233RZ, Samsung, Seoul, South Korea), with 1680 x 1050 pixels resolution and a refresh rate of 120 $\mathrm{Hz}$.

A centrally located white square (side length $0.2 \mathrm{dva}$, luminance $72 \mathrm{~cd} / \mathrm{m}^{2}$ ) was used as a fixation point. The endogenous pre-cue, drawn as a red isosceles triangle (side length $0.4 \mathrm{dva}$ ), pointing either to the left or to the right, was presented centrally replacing the fixation point. The stimuli used were white random dot patterns (RDP) with a density of $10 \mathrm{dots} / \mathrm{dva}^{2}$, moving with a speed of $8 \mathrm{dva} / \mathrm{sec}$. Each patch had a diameter of 5 dva centered at an eccentricity of 5 dva left or right on horizontal meridian. The dots' size was approx. 0.0564 dva. The coherence of the RDP was controlled by the proportion of the dots moving coherently in one direction. The remaining dots moved to all other directions with the same speed of $8 \mathrm{dva} / \mathrm{sec}$. All stimuli were presented on a black background with a luminance of $0.3 \mathrm{~cd} / \mathrm{m}^{2}$. 


\section{Design and Procedure}

Seated in a dark room, $57 \mathrm{~cm}$ from the monitor, the subjects were asked to initiate every trial by fixating the fixation point and pressing the start button on a commercial gamepad (Logitech International S.A., Switzerland). The trial sequence is shown in Figure 1a. Immediately after button press, the centrally presented fixation point was replaced by the endogenous pre-cue, pointing either to the left or to the right side, for 200 milliseconds (ms). After an inter stimulus interval of $300 \mathrm{~ms}$, two RDP stimuli were shown left and right with 5 dva eccentricity respectively to the central fixation point on the horizontal meridian. At any given trial, one of the two RDP contained the task-relevant event (target), a brief coherent linear motion signal ( $75 \mathrm{~ms}$ ), moving towards the fixation point. Depending on experimental condition, the motion coherence of the target stimulus was manipulated. The second RDP contained fully incoherent linear motion (distractor) moving to all possible directions. At target stimulus position, a random motion mask was shown for $75 \mathrm{~ms}$ to stop information uptake. At distractor location a static dot pattern was shown for the same duration, acting as a post-cue. Subjects were asked to indicate the location (left or right) and to discriminate the motion direction (up or down relative to horizontal) of the target stimulus by pressing one out of four buttons on a gamepad. The subjects were asked to report as accurate as possible the perceived direction of the target RDP without receiving any feedback about their decision. 
a)

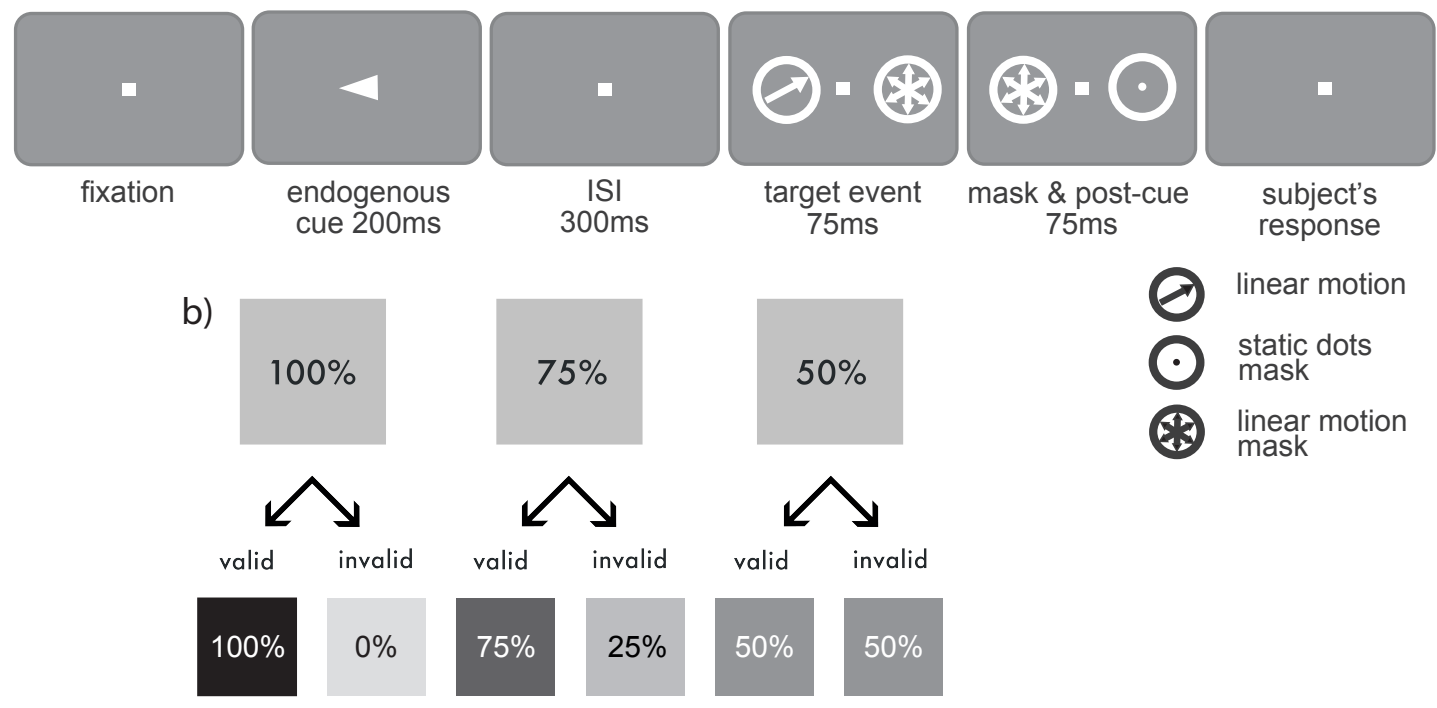

Figure 1. a) Experimental protocol. The time course of one trial in the experimental task is shown. An endogenous pre-cue, shown for a duration of $200 \mathrm{~ms}$, pointing either to the left or to the right and guides subject's spatial attention. After a delay of $300 \mathrm{~ms}$, target and distractor RDP appeared. In the target RDP dots move linearly towards the centrally presented fixation point. In the distractor RDP dots move to all possible directions. The target RDP was followed by a mask stimulus, whereas the distractor RDP was followed by a static dot pattern, serving as a post-cue. Subjects were instructed to report the position of the target dot pattern (left or right) and its linear motion direction (up or down based on horizontal meridian) by pressing one of four buttons on the gamepad (4-alternative forced choice design). b) 3 different validities $(100 \%, 75 \%$, and $50 \%)$ of the endogenous pre-cue were shown in a block wise manner. This leads to 4 levels of spatial attention (100\%, $75 \%, 50 \%$ and $25 \%)$.

Eye position of both eyes was monitored during the whole experiment, using an eye tracker (EyeLink, SR research). Each trial was aborted when subjects did not maintain fixation at the fixation point during stimulus presentation.

Endogenous spatial attention was manipulated by presenting three different levels of pre-cue validity (100\%, $75 \%$ and $50 \%$ ) in a block-wise manner. The subjects were informed about the cue-validity prior to each block and were asked to change their behavior accordingly. The three levels of cue validity resulted in four levels of spatial attention. Invalid trials in the $75 \%$ pre-cue validity condition are considered as an attentional deployment of $25 \%$. 
Additionally, the strength of the target's motion signal was block wise manipulated by varying the percent of coherently moving dots, resulting in four levels of motion coherence $(40 \%, 60 \%, 80 \%, 100 \%)$.

All cue validity conditions and motion coherence conditions were combined, resulting in a total amount of 12 blocks. Each block comprised 320 trials. The blocks with $75 \%$ pre-cue validity were performed twice, in order to collect an appropriate amount of data points for the invalid trials (25\% of spatial attention).

Subjects underwent three practice sessions followed by four experimental sessions, all performed on consecutive days. Within one session four blocks were performed. Across experimental sessions the order of experimental blocks was randomized.

\section{Training of observers}

Subjects ran through an intensive training, consisting of at least three training sessions (1.5 h duration), performed on three consecutive days. They performed all condition combinations, 12 blocks in total, with at least 3840 trials. The purpose of the training was to familiarize the subjects with the different conditions of the task design as well as to judge their performance and avoid an improvement in performance due to learning effects for the following experimental sessions. After the training sessions the subjects had to reach a minimum criterion of performance (4 deg discrimination threshold in 100\% cue validity and $100 \%$ motion coherence condition) in order to be included in the following experimental sessions. Data collected in the training sessions were excluded from the main analysis. 


\section{Staircase procedure}

The shown linear motion direction in the target dot pattern was varied dynamically using interleaved staircases, achieving a rational amount of repetitions close to the point of subjective equality (PSE). In total, four randomly interleaved staircases were used to manipulate linear motion direction in the target dot pattern. They always started at a fixed level of $5 \mathrm{deg}$ above and below horizontal (o deg), changing with a factor of 1.25. In detail, four staircases were active for one RDP. Two staircases acted when a valid precue was shown, whereas two independent staircases acted when there was an invalid pre-cue. All staircases followed a 2 to 1 rule, converging when observers' answer was twice consecutive consistent with shown direction and diverging when the answer was inconsistent. An example action of the four randomly interleaved staircases across one experimental block is shown in supplementary Figure 1.

\section{Data analysis}

The task was designed to gradually manipulate the allocation of spatial attention of the subject when various stimulus strengths were used. By using block wise different cue validity levels the subjects were instructed to change behavior accordingly.

Psychometric functions were fitted for behavioral data recorded at both spatial locations (left and right) and for valid and invalid trials separately, using a maximum likelihood procedure combined with bootstrap sampling (Palamedes Toolbox, Kingdom \& Prins, version 1.8.1). A Cumulative Normal function was used to model the psychometric data. 
The Cumulative Normal distribution is given as:

$F_{N}(x ; \alpha, \beta)=\frac{\beta}{\sqrt{2 \pi}} \int_{-\infty}^{x} \exp \left[-\frac{\beta^{2}(x-\alpha)^{2}}{2}\right]$,

where parameter $x$ is the signal strength, $\alpha$ corresponds to the threshold and $\beta$ corresponds to the reciprocal of the standard deviation of the normal distribution and determines the slope.

The direction discrimination threshold was defined as the first deviation of the fitted psychometric function and calculated for every experimental condition as well as for cue validity separately. It served as a measure of the change in performance with changing cue validity and motion coherence. The corresponding confidence interval for each slope parameter was calculated using parametric bootstrapping (Palamedes Toolbox, Kingdom \& Prins, version 1.8.1). Direction discrimination thresholds for left and right position were merged for each experimental condition by calculating the weighted mean, using the inverse of the $95 \%$ confidence interval as a weight factor. Across subjects, the weighted mean was again calculated.

Four subjects were excluded, because bootstrap parametric did not converge for individual experimental conditions and therefore the direction discrimination threshold could not be reliably calculated. The direction discrimination thresholds for the remaining 16 subjects were compared with a repeatedmeasures ANOVA using Statistica software (Statistica 12, StatSoft). Effects of different attentional levels and different stimulus coherence levels were compared with a $4 \times 4$ repeated measures ANOVA with attention and stimulus coherence as main factors. Post-hoc comparison was performed using Least Significant Difference- Bonferroni Test, correcting for multiple comparisons.

With our task design we were able to distinguish between two types of errors, location errors and discrimination errors. In the case where the subjects answered at distractor location, it is assumed that the linear movement at target 
location was not perceived at all. Therefore these trials were excluded from further analysis. In the other case, where the subjects answered at the correct location (target), all trials were incorporated in further analysis.

\section{Results}

With our task design we aim to gradually manipulate spatial attention across two locations. Spatial attention was manipulated by presenting three different levels of pre-cue validity. Additionally, we manipulated the signal strength of the target dot pattern by varying the percentage of coherently moving dots. The direction discrimination threshold serves as a measure of the change in performance with changing cue validity and motion coherence.

In a first step, we investigated the impact of spatial attention on performance when the signal strength was at its maximum (100\% coherence). In Figure 2 the weighted mean of the direction discrimination thresholds across 16 subjects is shown for all four attentional conditions. 


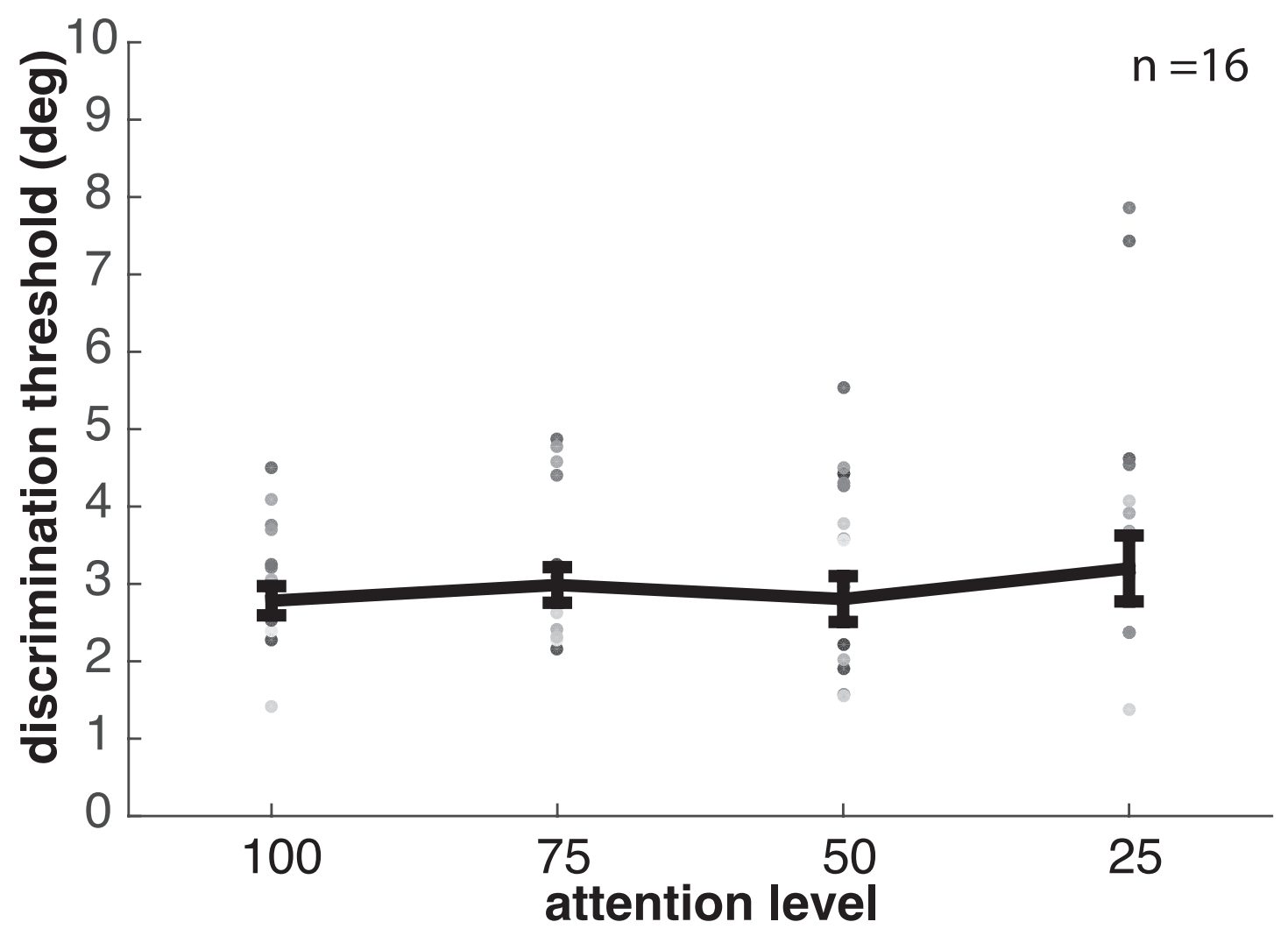

Figure 2. Individual and average result of 16 subjects of the spatial attention task, when target dot pattern had maximum signal strength. The weighted mean of the direction discrimination threshold was calculated across subjects with the inverse of the $95 \%$ confidence interval serving as the weight factor. Error bars indicate the standard error of mean.

When fully attending one spatial location, subjects were able to discriminate a change of $2.8 \pm 0.19 \mathrm{deg}$ (standard error of mean, SEM) in linear movement. The weighted mean across subjects increased marginally with decreasing amount of deployed spatial attention, $3.0 \pm 0.23,2.8 \pm 0.29$ and $3.2 \pm 0.43$ for $75 \%, 50 \%$ and $25 \%$ attentional condition respectively, showing no significant change (repeated-measures ANOVA with post-hoc least significant DifferenceBonferroni Test; main effect of attention $F(3,45)=17.8, p=0.099)$. The result is in line with the result attained in the previous manuscript, showing no effect 
on performance when various amounts of attention are deployed to two locations distributed across the two hemifields.

In contrast to the absent influence of cue validity on performance, the change in target stimulus coherence had a significant effect on subjects' direction discrimination (rmANOVA, $F(3,45)=7.86, p<0.001$ ). The first series of figure 3 (black bars) depicts the influence of signal strength when spatial attention was at its maximum.

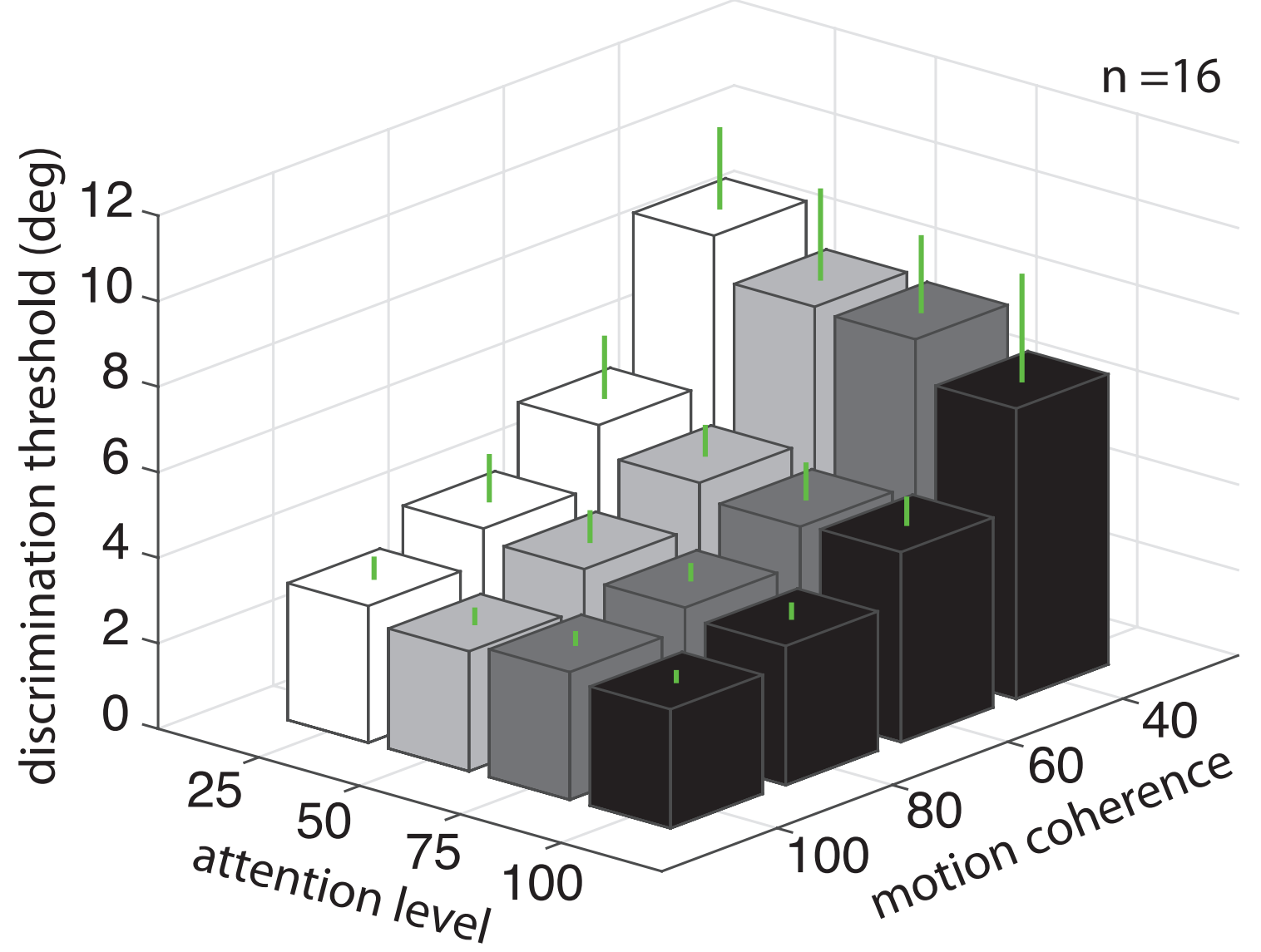

Figure 3. Weighted averaged direction discrimination thresholds for all attention and motion coherence conditions. Error bars indicate the standard error of the mean. 
Here, a gradual decrease in signal strength induced a gradual increment in discrimination threshold, resulting in a 2-fold bigger discrimination threshold for the weakest signal strength (40\% coherence) compared to full signal strength (100\% coherence). A decrement of $20 \%$ in signal strength, from $100 \%$ to $80 \%$ coherence, did not lead to a significant change in performance ( $p=o .61)$. A further reduction in signal strength, however, influenced the performance in direction discrimination significantly $(60 \%$ and $40 \%$ coherence, both $p<0.0001)$. Overall, the reduction from $60 \%$ of coherently moving dots to $40 \%$ showed strongest effect on perceptual performance (4.4 deg to $6.7 \mathrm{deg}$ ).

In addition to an individual influence of the two main factors, stimulus strength and spatial attention, we were interested in their collective influence on perceptual performance. Therefore all attentional conditions were combined with all stimulus coherence conditions. Figure 3 summarizes the effect sizes for all experimental conditions, showing the weighted mean with the corresponding SEM for all possible task combinations. No interaction was found between the two main factors on direction discrimination $(F(9,135)=1.67, p=0.19)$, suggesting that the direction discrimination threshold varies independently as a function of attention and stimulus strength.

In order to further investigate the influence of attention for the different stimulus strengths, we normalized the direction discrimination thresholds to the thresholds in $100 \%$ attention condition to compensate for inter-subject variability. 


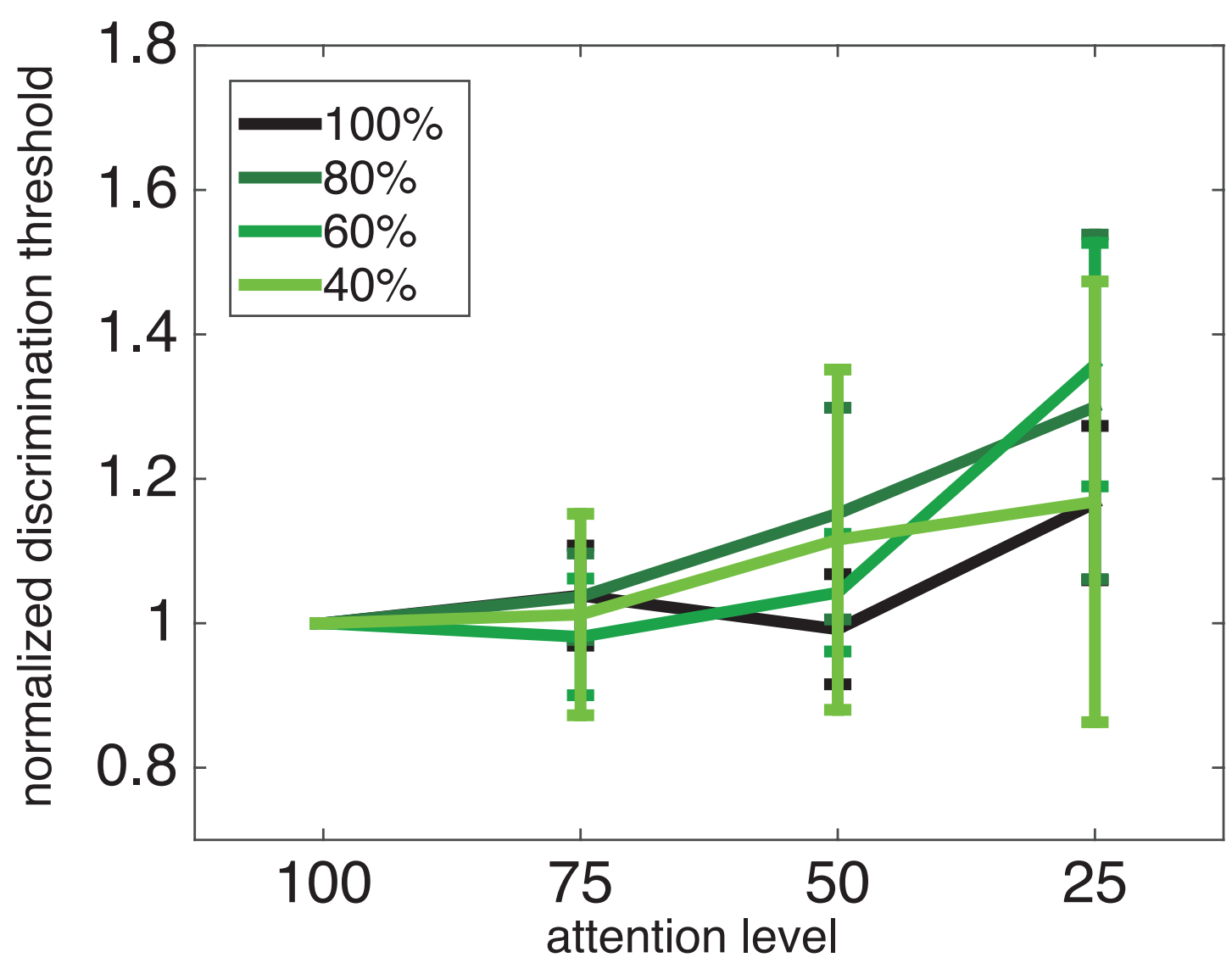

Figure 4. Normalized direction discrimination threshold. All direction discrimination thresholds were normalized to the $100 \%$ attention condition for all four stimulus coherence levels separately. Error bars indicate the standard error of the mean.

Figure 4 depicts the relative change of direction discrimination with respect to the $100 \%$ attention condition for all stimulus coherence levels separately. Independent of signal strength, the direction discrimination was influenced in the same manner, showing no significant difference across attention conditions ( $r m A N O V A, ~ F(3,240)=0.16, p=0.92)$.

With our task design we are able to eliminate the spatial uncertainty as a critical factor from our results as we are able to distinguish location errors from discrimination errors. Therefore, only answers at the target location were 
incorporated in previously described analysis and answers at distractor location were excluded.

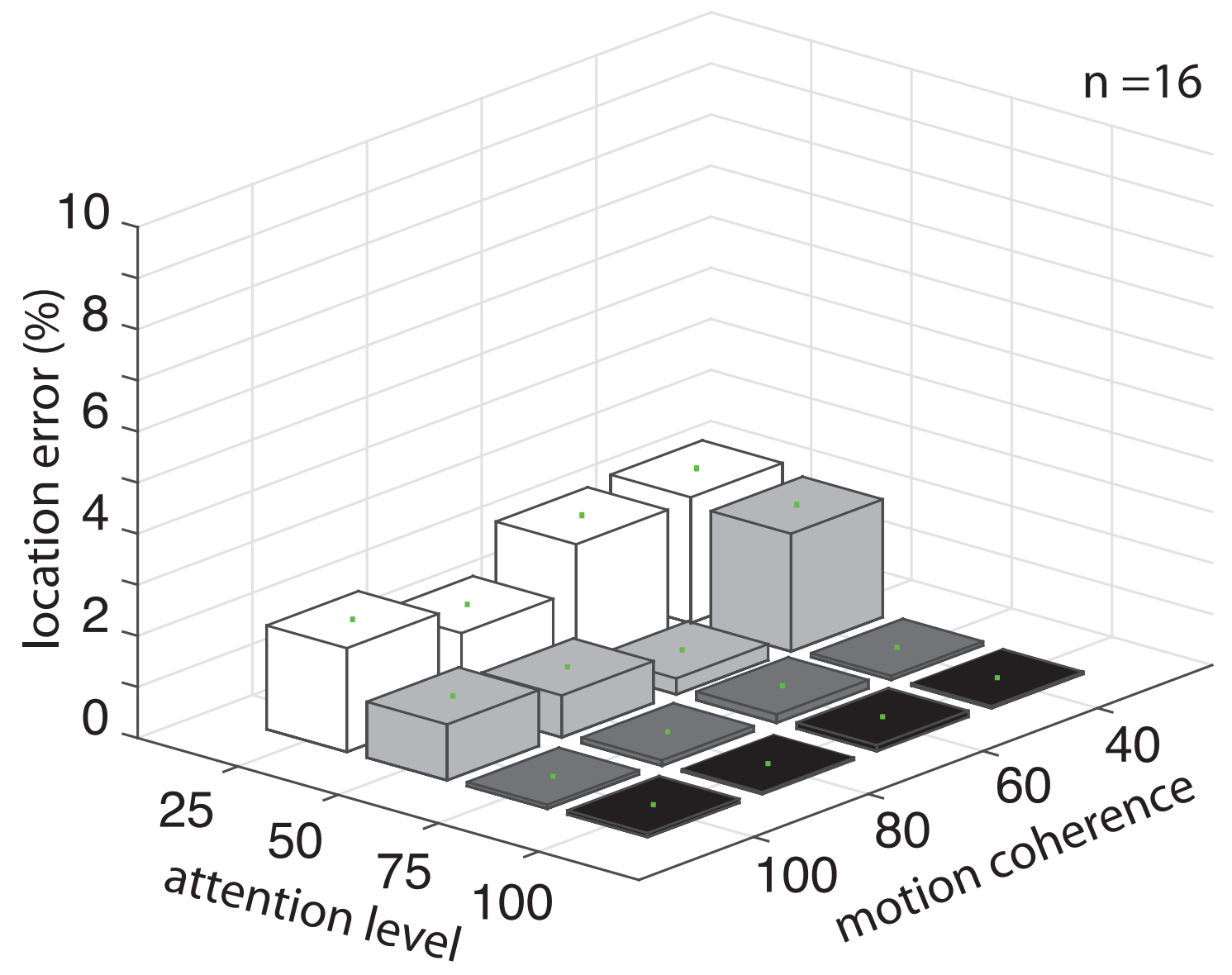

Figure 5. Percentage of ignored trials for all experimental conditions. Trials were considered as ignored, when the answer was given at distractor location.

Figure 5 shows the percentage of location errors for all experimental conditions. Although the location errors emerged more often for lower attentional conditions, their magnitude never exceeded $2 \%$ of all trials.

As we aim to motivate the usage of weighted values in order to deal with variances and to avoid erroneous conclusions, we calculated and compared the arithmetic means for all experimental conditions (see Figure 6). 


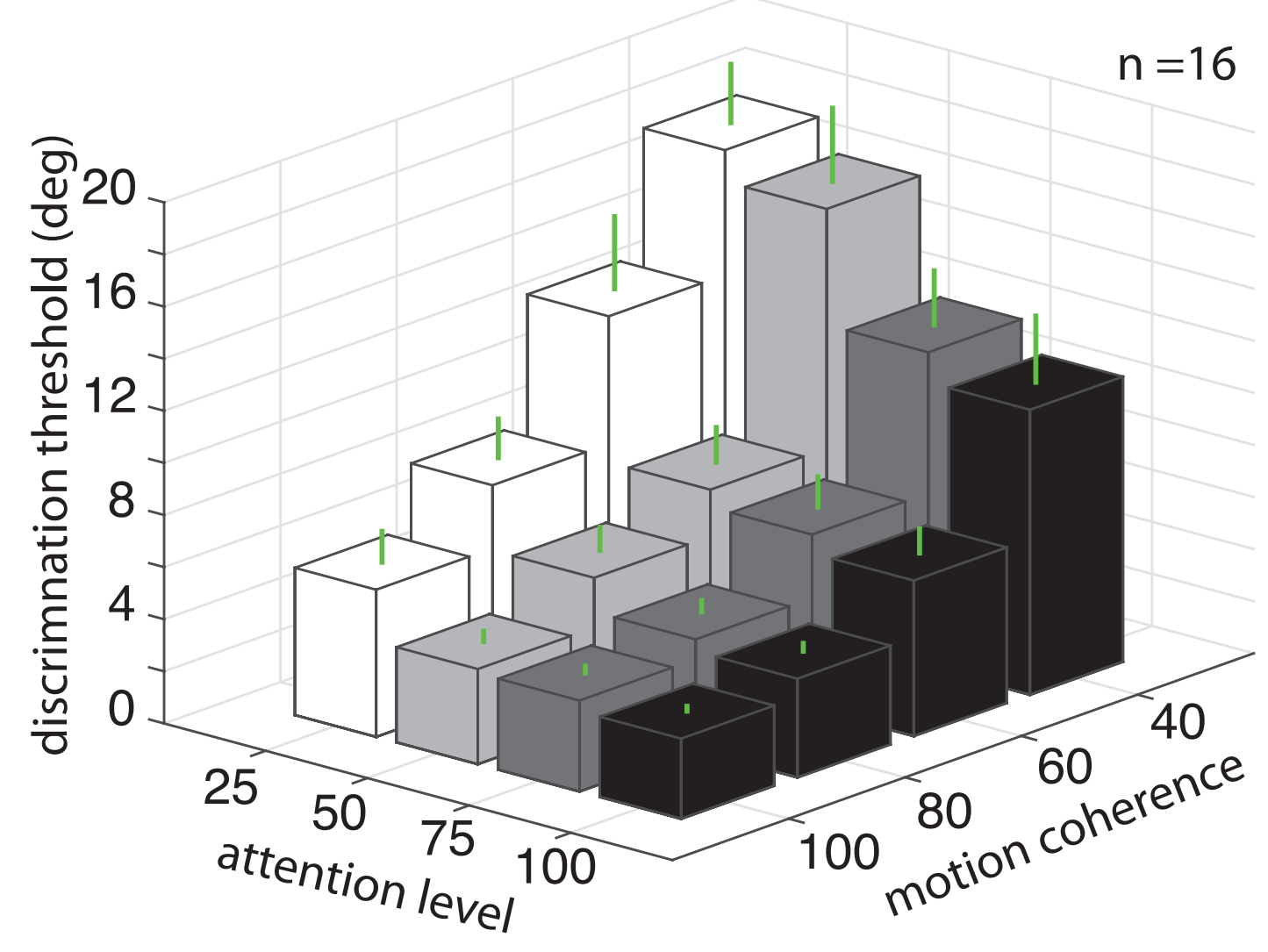

Figure 6. Arithmetic means of the direction discrimination thresholds across 16 subjects. Error bars depict standard error of means.

The analysis model, based on a repeated-measures ANOVA, showed a significant effect of the two main factors, attention $(F(3,45)=5.8, p=0.01)$ and coherence $(F(3,45)=23.1, \quad p<0.01)$, on to the direction discrimination threshold, whereas no interaction was found $(F(9,135)=0.47, p=0.89)$. Overall, the mean discrimination thresholds were considerably higher compared to the weighted mean values. Especially the lowest level of attentional deployment (25\%) was remarkably higher when calculating the arithmetic mean, e.g. $18 \mathrm{deg}$ for $40 \%$ coherent stimuli compared to 8 deg for the weighted mean. Post-hoc comparison revealed significant difference between $25 \%$ of attentional deployment compared to $75 \%$ and $100 \%$ of attentional deployment. This effect 
was eliminated, when the mean was weighted with the inverse of the 95\% confidence interval serving as the weight factor, indicating that is effect is strongly influenced by extreme values having big uncertainties.

\section{Discussion}

Here we investigate the effects of spatial attention on performance in a direction discrimination task using moving random dot patterns of various signal-tonoise ratios. This allowed us to equate the effects of attention with the effects of stimulus coherence on perceptual performance. We gradually manipulated the relevance of one spatial location with respect to a second spatial location by varying the validity of an endogenous pre-cue, resulting in four level of spatial attention (100\%, 75\%, 50\% and 25\%). Additionally, the signal strength of the target dot pattern was varied by controlling the proportion of the dots moving coherently in one direction, achieving four levels of motion coherence $(100 \%$, $80 \%, 60 \%$ and $40 \%)$. The effect on performance of both factors, attention and stimulus coherence, were measured calculating the discrimination threshold for linear motion.

Observing graded behavioral improvements in correlation with spatial specificity would support the existence of limited attentional resources, that would need to be partitioned in order to optimally process stimuli at multiple locations (Kahneman, 1973; Broadbent, 1971). In our task design performance wasn't significantly affected when one stimulus was fully attended, compared to when attention needed to be split across two spatial locations. In other words, judging a linear movement at two locations at the same time did not incorporate a noteworthy cost compared to a judgment at just one location. Surprisingly, even when the amount of deployed spatial attention was further diminished to a level of $25 \%$, no significant decrement in performance was observed. This 
observation is in accordance with the results of the first psychophysical study. But this result does not support results gained in other psychophysical studies, showing an improvement in motion processing with provided spatial information (Liu, Fuller \& Carrasco, 2006; Verghese, Anderson \& Vidyasagar, 2013). However, there are also other studies showing that attention had little or no effect on performance when the subjects had to perform motion discrimination (Lee et al., 1997, Lee et al., 1999). These studies claim that the attentional demands for motion discrimination are fairly low, compared to other tasks such as letter discrimination (Braun, 1994; Braun \& Julesz, 1998). It can be therefore argued, that the judgment of linear motion is possibly performed in absence of attention. Alternatively, it is assumed that there is some amount of visual awareness outside the attentional spotlight, permitting the subject to perform a task with low attentional cost equally well for stimuli at locations inside and outside of the attentional spotlight (Braun and Julesz, 1998).

We deliberately chose stimuli parameters to comprise attentional demands. Consequently we chose a very short stimulus presentation of $75 \mathrm{~ms}$, followed by a mask to stop information uptake. Additionally, we dynamically varied the shown linear motion direction in the target dot pattern depending on subject's performance, in order to target small angles of linear motion direction. We can further exclude that subjects perform a serial shift of spatial attention across the two locations, as our short task design timing doesn't allow for such changes (Reeves \& Sperling, 1986, Duncan et al., 1994).

Based on results gained in the former study, a possible explanation for the lack of attentionally guided improvement is that the two attended locations are distributed along the horizontal line, thus targeting independently working attentional resources or mechanisms for the two stimuli (Alvarez and Cavanagh, 2005). 
Moreover, we were especially interested in whether varying spatial attention would have an equivalent influence on behavior compared to varying the signal strength. Physiological studies suggested already, that attentional deployment leads to a multiplicative change in neuronal response without influencing the neuron's stimulus selectivity (Treue \& Martinez- Trujillo, 1999; McAdams \& Maunsell, 1999). Therefore the same neuronal response can be elicited by increasing the stimulus strength such as motion coherence (Sclar \& Freeman, 1982). However, a recent study contrasting attentional influence on behavioral performance with attentional influence on neuronal response did not report any correlation and suggests that attention acts as a regulatory component. In this framework, attention is thought to alter the relationship of neuronal response and behavioral performance and therefore limits the neuronal response's power in predicting the behavior (Cook \& Maunsell, 2002).

In this study we observed no influence of attention onto behavioral performance, whereas the signal strength showed strong and graded influence. We therefore cannot support in our task design, that attention would act in a manner similar to varying stimulus strength.

Our paradigm allowed us additionally to measure the interactive influence of both factors on perceptual performance. As earlier behavioral studies revealed that attention enhances the subjective appearance of motion coherence (Liu et al., 2006), we expected to observe an interaction of both factors onto perceptual performance. However, we did not find such interaction. We could demonstrate, that a reduction in signal strength did not increase the attentional demand. On the basis of these results, we claim that either spatial attention and signal strength co-act as independent systems on the subjects' perception of linear motion, or due to the absence of attentional influence in this task design a correlation could not be determined.

As attentional capacity limits were possibly not reached with this across hemifield task design, it would be of interest to investigate the influence of 
signal strength and attentional deployment on behavioral performance when both stimuli are placed within one hemifield.

\section{References}

Alvarez GA, Cavanagh P (2005) Independent resources for attentional tracking in the left and right visual hemifields. Psychol Sci. 16(8):637-43.

Bonnel AM, Miller J (1994) Attentional effects on concurrent psychophysical discriminations: Investigations of a sample size model. Perception \& Psychophysics 55:162-179.

Bonnel AM, Prinzmetal W (1998) Dividing attention between the color and the shape of objects. Perception \& Psychophysics 60:113-124.

Bosworth RG, Petrich JA, Dobkins KR (2012) Effects of spatial attention on motion discrimination are greater in the left than right visual field. Vision Research 52:11-19.

Braun J (1994) Visual search among items of different salience: Removal of visual attention mimics a lesion in extrastriate area V4. Journal of Neuroscience 14:554-567.

Braun J, Julesz B (1998) Withdrawing attention at little or no cost: Detection and discrimination tasks. Perception \& Psychophysics 60:1-23.

Britten KH, Shadlen MN, Newsome WT, Movshon JA (1992) The analysis of visual motion: A comparison of neuronal and psychophysical performance. The Journal of Neuroscience 12:4745-4765.

Broadbent DA (1971) Decision and stress. London: Academic Press.

Carrasco M (2011) Visual attention: the past 25 years. Vision Res 51: 1484-1525. 
Cook EP, Maunsell JH (2002) Attentional Modulation of Behavioral Performance and Neuronal Responses in Middle Temporal and Ventral Intraparietal Areas of Macaque Monkey. The Journal of Neuroscience 22(5):1994-2004.

Duncan J (1984) Selective attention and the organization of visual information. Journal of Experimental Psychology: General 113:501-517.

Duncan J, Ward R, Shapiro K (1994) Direct measurement of attentional dwell time in human vision. Nature 369(6478):313-5.

Kahneman D (1973) Attention and effort. Englewood Cliffs, NJ: Prentice-Hall.

Lee DK, Koch C, Braun J (1999) Attentional capacity is undifferentiated: concurrent discrimination of form, color, and motion. Percept Psychophys. 61(7):1241-55.

Lee DK, Koch C, Braun J (1997) Spatial vision thresholds in the near absence of attention. Vision Research 37:2409-2418.

Liu T, Fuller S, Carrasco M (2006) Attention alters the appearance of motion coherence. Psychonomic Bulletin \& Review 13(6):1091-1096.

Luck SJ, Hillyard SA, Mouloua M, Woldorff MG, Clark VP, Hawkins HL (1996) Effects of spatial cuing on luminance detectability: psychophysical and electrophysiological evidence for early selection. Journal of Experimental Psychology: Human Perception and Performance 20(4):887-904.

McAdams CJ, Maunsell JHR (1999) Effects of attention on orientation tuning functions of single neurons in macaque cortical area V4. The Journal of Neuroscience 19:431-441.

Pashler HE (1998) The Psychology of Attention. Cambridge, MA: MIT Press.

Posner, MI (1980) Orienting of attention. The Quarterly journal of experimental psychology $32(1): 3-25$. 
Prins N, Kingdom FAA (2009) Palamedes: Matlab routines for analyzing psychophysical data. http://www.palamedestoolbox.org

Reeves A, Sperling G (1986) Attention gating in short-term visual memory. Psychol Rev. 93(2):180-206.

Sclar G, Freeman RD (1982) Orientation selectivity in the cat's striate cortex is invariant with stimulus contrast. Exp Brain Res. 46:457-461.

Treue S, Maunsell JH (1996) Attentional modulation of visual motion processing in cortical areas MT and MST. Nature 382(6591):539-41.

Treue S, Martinez Trujillo JC (1999) Feature-based attention influences motion processing gain in macaque visual cortex. Nature 399:575-579.

Verghese A, Anderson AJ, Vidyasagar TR (2013) Space, color, and direction of movement: how do they affect attention? Journal of Vision 13(8):20, 1-8.

Wilimzig C, Tsuchiya N, Fahle M, Einhäuser W, Koch C (2008) Spatial attention increases performance but not subjective confidence in a discrimination task. J Vis. 8(5):7.1-10. 
Supplementary figures:
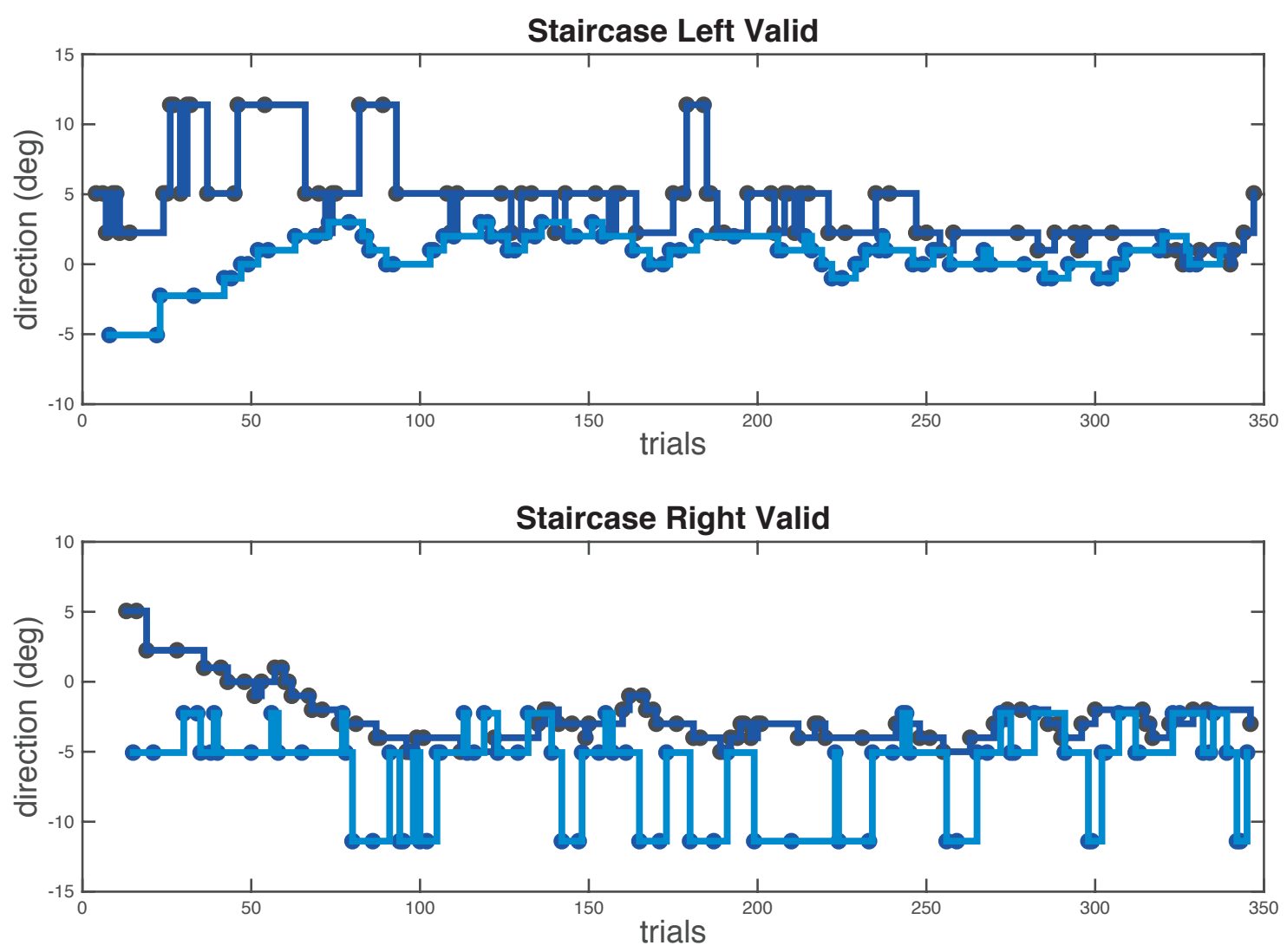

Suppl. Figure 1. Activity pattern of staircases across an experimental block. For each stimulus position (left and right) and for each validity condition (valid and invalid), two independent staircases (dark blue and light blue) were active. The upper plot depicts the staircase values (linear movement directions in deg) for the two staircases for the left valid stimulus; the lower plot depicts the two staircases for the right valid stimulus. Staircases always started at a fixed level of 5 deg above and below horizontal (o deg), changing with a factor of 1.25. All staircases followed a 2 to 1 rule, converging when observers' answer was twice consecutive consistent with shown direction and diverging when the answer was inconsistent. Interleaved staircases were used in order vary dynamically the shown linear motion direction in the target dot pattern, achieving a rational amount of repetitions close to the point of subjective equality (PSE). 


\title{
Video Article \\ A Pressure Injection System for Investigating the Neuropharmacology of Information Processing in Awake Behaving Macaque Monkey Cortex
}

\author{
Vera Katharina Veith ${ }^{1}$, Cliodhna Quigley ${ }^{1}$, Stefan Treue ${ }^{1}$ \\ Cognitive Neuroscience Laboratory, German Primate Center \\ Correspondence to: Vera Katharina Veith atVVeith@dpz.eu URL: http://www.jove.com/video/53724 \\ DOI: doi:10.3791/53724
}

Keywords: visual spatial attention, macaque monkey, pressure injection, scopolamine, extracellular recording, electrophysiology, microinjection, awake behaving animal, drug delivery

Date Published: 12/21/2015

Citation: Veith, V.K., Quigley, C., Treue, S. A Pressure Injection System for Investigating the Neuropharmacology of Information Processing in Awake Behaving Macaque Monkey Cortex. J. Vis. Exp. (), e53724, doi:10.3791/53724 (2015).

\begin{abstract}
The top-down modulation of feed-forward cortical information processing is functionally important for many cognitive processes, including the modulation of sensory information processing by attention. However, little is known about which neurotransmitter systems are involved in

such modulations. A practical way to address this question is to combine single-cell recording with local and temporary neuropharmacological manipulation in a suitable animal model. Here we demonstrate a technique combining acute single-cell recordings with the injection of neuropharmacological agents in the direct vicinity of the recording electrode. The video shows the preparation of the pressure injection/recording system, including preparation of the substance to be injected. We show a rhesus monkey performing a visual attention task and the procedure of single-unit recording with block-wise pharmacological manipulations.
\end{abstract}

\section{Video Link}

The video component of this article can be found athttp://www.jove.com/video/53724/

\section{Introduction}

In cortical and subcortical areas, neuronal activity is affected by various neuromodulators, for example acetylcholine ${ }^{1}$. These modulatory effects on neuronal responses have been reported in in vitro studies ${ }^{2}$, as well as in electrophysiological recordings from anesthetized animals ${ }^{3}$ and systemic pharmacological manipulations in humans ${ }^{4}$. Nevertheless, the exact role of different neuromodulators and the involvement of various receptor subtypes are largely unknown. To measure the effects of specific neuromodulators on the activity of single neurons, it is desirable to induce a temporary neuromodulator change as close as possible to the recording electrode. Furthermore, it is important that those

manipulations are done in awake animals, as cognitive functions are only present in the absence of anesthesia. Additionally, anesthesia interacts with cholinergic and GABAergic systems ${ }^{5,6}$ and can lead to changes in neural activity $^{3}$.

Within the last decades, two main methods of local drug delivery have been developed and refined: iontophoresis and pressure injection. In both methods drugs are delivered through micropipettes made of either glass or steel. With iontophoresis, an electrical current regulates the release of the $\mathrm{drug}^{7}$. Additionally, there is a significant contribution of electro-osmosis to the total amount of ejected molecules $^{8}$, correlating with the tip diameter ${ }^{9}$ of the micropipette as well as with the concentration ${ }^{8}$ of the substance used. Iontophoresis is a powerful tool to quickly and precisely manipulate small volumes of nervous tissue. For iontophoretic injections, multi-barrel micropipettes are usually used ${ }^{10}$, with one acting as a recording device while the other positions serve as delivery pipettes. A limitation of this method is that only charged molecules can be used, severely limiting the selection of drugs. 
Pressure injection uses either air compression or mechanical pressure to eject a substance from a micropipette. Using this method any soluble substance, charged or uncharged, can be used, including large molecules. The method of pressure injection was first described by Reyniers

in 1933 and further refined in the 1950 s (see Lalley ${ }^{11}$ for a review). In the 1980 s the method was further refined to allow delivery of amounts in

the nanoliter range (mainly lidocain ${ }^{12}$ ) to a defined brain area ${ }^{13}$ while simultaneously performing single-cell recording. The ejected volume was usually monitored by observing the movement of a marker, such as the meniscus in the upper part of the pipette ${ }^{13}$. Pressure injection was first used in the 1990 s in awake animals, both extracellularly ${ }^{14}$ and intracellularly ${ }^{15,16}$. Based on the cumulative expertise gained in these studies it is now possible to reliably record from different brain structures in combination with pharmacological manipulation (see 17 for a comparison of recent pressure injection systems).

An enduring open issue for both drug delivery methods is the difficulty in determining the precise volume injected. This is an even bigger challenge for experiments with awake, behaving rhesus monkeys where the animal performs the experimental task in a separate room. This can be alleviated by the use of a software-controlled system instead of relying on a visual marker to continuously monitor an injection.

The system described here is an extension of a well-established electrophysiological recording system (Mini Matrix System) and combines an injection pipette with multiple parallel-oriented recording electrodes at defined distances in a customizable arrangement. Pharmacological

manipulation of the tissue near the recording electrode is possible using only a small amount of substance, ensuring a fast recovery and allowing multiple blocks of injection and control/recovery within the limited time window offered by the behavioral task of the animal. 


\section{Protocol}

Animal care and all experimental procedures were conducted in accordance with German laws governing animal care and approved by the district government of Braunschweig, Lower Saxony, Germany.

Note: As the experiment is performed in vivo, it is crucial to maintain the highest possible hygiene standards. Whenever possible, work under sterile conditions.

\section{Preparing the injection/recording system}

1. Sterilize the tube that connects the micropipette with the syringe. Use the shortest length of tube possible in the experimental set-up between injection pump and electrophysiological recording system.

2. Clean the guide tubes of the recording system using cleaning wires. Dip them in sterile silicon oil and feed them through the individual guide tubes several times.

3. Insert the quartz glass micropipette into one guide tube of the recording system. See Figure 1a.

4. After fixing the micropipette in the recording system, attach the sterile tube to the metal pin of the micropipette. Take care; although the micropipette is fixed in the system it can easily break when attaching the tube. Use two sterile tweezers to apply equal pressure on pin and tube.

5. Use liquid super glue to seal the junction between tube and micropipette. Wait at least 3 hours for the glue to harden before filling the micropipette with liquid.

6. Insert microelectrodes (e.g. quartz glass insulated platinum tungsten) into the other positions of the recording system before or after micropipette insertion.

\section{Preparing the substance}

1. Sterilize $1.5 \mathrm{~mL}$ microcentrifuge tubes for later storage of injection solutions using an autoclave or other reliable procedure.

2. Weigh the corresponding substance scopolamine hydrochloride to prepare $5 \mathrm{~mL}$ of a 0.1 molar solution. Dissolve in sterile saline $(0.9 \% \mathrm{NaCl})$.

3. Under sterile conditions in a fume hood, filter the solution using a syringe filter with sufficiently large pore diameter, e.g. $0.2 \mu \mathrm{m}$.

4. Under the fume hood, aliquot the solution into volumes sufficient for a single experiment, e.g. for scopolamine, 500 $\mu \mathrm{L}$ in sterile $1.5 \mathrm{~mL}$ microcentrifuge tube. Use dark tubes to protect the substance from light; alternatively, wrap tubes in aluminum foil. For scopolamine, store the solution for up to 14 days at $4{ }^{\circ} \mathrm{C}$.

\section{Daily preparation of the injection/recording system}

Note: When mounted in the recording system, the electrodes and micropipette are stored in an enzyme solution (Tergazyme, $1 \%$ solution with deionized water) between recordings. The following steps must be performed before every recording.

1. Collect a tube of the substance to be injected. Allow it to reach room temperature if refrigerated.

2. Remove the injection/recording system from the enzyme solution and rinse electrodes and micropipette with deionized water to clean completely of enzyme solution.

3. Remove the front cover of the recording system in order to visually check the seal between micropipette and tube.

4. Apply sterile silicon oil to the guide tube gap (see Figure 1a) and tips of electrodes and micropipette in order to lubricate the systemfor smooth movement.

5. Check tips of electrodes and micropipette using a microscope to ensure they are intact. Align the electrodes and the micropipette under the microscope so that they extend out of the guide tubes with the same length. Drive them into the guide tubes, stopping as soon as they are no longer visible. This is defined as electrode position zero. Set the depth of the electrodes and micropipette to 0 in the software.

6. Fill a sterile syringe with sterile saline and insert the needle into the tube, taking care not to pierce the wall of the tube. Drive the micropipette out of the guide tube for visual control of substance flow.

7. Flush at least $2 \mathrm{~mL}$ sterile saline through the tube and micropipette to ensure no air remains in the syringe or in the tube. Do not apply too much pressure to the plunger of the syringe. Ensure the junction between tube and micropipette is sealed. If leakage is visible, re-glue the junction (see step 1.5) and postpone recording.

8. Fill a new sterile syringe with the solution to be injected and exchange it with the barrel of the saline syringe, i.e. keep the needle of the saline-filled syringe in the tube. Make sure that no air is transferred into the system. This is best achieved by filling the needle hub with saline after removing the saline-filled barrel.

9. Flush the system with $250 \mu \mathrm{L}$ of the solution to be injected, in order to completely remove the saline from the tube.

10. Using the motor control software, retract electrodes and micropipette into the guidetubesto a depth of at least $-500 \mu \mathrm{m}$

11. Lower the guide tube ring (Figure 1a) to the bottom of the guide tubes to maintain their fixed relative position.

12. Clean the base of the system with ethanol, in particular where it will touch the monkey's recording chamber.

13. Close the recording system by replacing the front cover and tightening the screws. 


\section{Validation of the injection system}

Note: Although the company calibrates the system, it is recommended to validate the ejected volumes with the materials used in the experimental set-up (tubes, syringes etc.).

1. Prepare the system as described in step 3, keeping electrodes and micropipette extended out of the guide tubes. A depth of at least $7000 \mu \mathrm{m}$ is recommended to avoid loss of measurement volume due to adhesion along the outer surface of the micropipette and electrodes.

2. Place the recording system in the position it will be used in during the experiment and put the syringe into the microinjection pump. Fix the syringe in place using the rubber band and adjustable grip (see Figure 1a). Slide the movable part of the pump until it is firmly in placebehind the plunger of the syringe.

3. Using the software-controlled motor unit, eject a volume large enough to be measured precisely, e.g. $1000 \mathrm{~nL}$. It is preferable to use one single step to eject the total volume in order to avoid effects of capillary action along the micropipette surface. Very low velocities $(1 \mathrm{~nL} / \mathrm{s})$ can also lead to this effect during the validation procedure.

4. Collect the total volume in a container placed under the micropipette, or carefully collect the ejected drop directly from the tip of the micropipette. Estimate the ejected volume using a pipette or by weighing with a precision scale.

5. Repeat the procedure several times to confirm measurements.

\section{Acute recordings}

1. Set the $x-y$ position of the recording system. This defines the point at which the guide tubes reach the dura mater within the chronically implanted recording chamber. Make sure the guide tubes are retracted completely (guide tube z position 0 ).

2. Bring the recording system into position and place the syringe in the microinjection pump. Fix the syringe in place using the rubber band and adjustable grip (see Figure 1a). Slide the movable part of the pump until it is firmly in place behind the plunger of the syringe. If a drop of substance is visible at the tip of the guide tubes, carefully remove it using a sterile cotton bud.

3. Prepare the animal for recording according to the laboratory's procedure (see ${ }^{18}$ for example guidelines).

4. Securely mount the recording system on the recording chamber of the monkey.

5. Slowly manually lower the guide tubes into the recording chamber until the dura is reached, then drive the electrodes using the motor control software.

6. As it is not possible to measure impedance of the micropipette, first drive with electrodes and check their impedances regularly atdifferent depths. After a penetration of the dura is successfully performed without damaging the electrodes, advance the micropipette.

7. Drive the electrodes and the micropipette to the target electrode depth at which the brain area of interest is expected to be found. Slowly advance the electrode until it is close enough to record the activity of a single unit, as evidenced by a good signal-to-noise ratio in the recorded signal. Importantly, position the recording electrode and the micropipette at the same depth to ensure the minimum distance between electrode and micropipette.

1. If possible, keep the electrodes and micropipette at this depth for the entire recording. However, if the only way to maintain signal quality of the recorded cell is to move the electrodes, then drive the electrodes and the micropipette simultaneously to maintain the distance between them.

\section{Spatial attention task}

1. In a series of trials, present two moving dot patterns on the screen, one positioned within the receptive field of the recorded neuron and the other outside of it, together with a centrally presented fixation point that the animal has to foveate throughout each trial ${ }^{19,20}$

Note: The monkey is trained to respond to a direction change in the cued dot pattern (the target event) while ignoring any direction change in the other dot pattern, and is rewarded with a drop of liquid for every successful completion of a trial ${ }^{19,20}$. As a sensory control condition, the monkey has to report a luminance change of the fixation point while ignoring both moving dot patterns (see Figure 2 for a more detailed description of the task). 


\section{Pharmacological manipulation while recording}

Note: While the monkey is performing the task, inject the substance in a block-wise manner. Three consecutive blocks are defined: control, which acts as a baseline; injection, during which a substance is ejected; and recovery, during which the cells targeted by the injection return to baseline.

1. During an injection block, inject a predefined amount of the substance at regular intervals e.g. $2 \mathrm{~nL}$ every minute at a rate of $2 \mathrm{~nL} / \mathrm{s}$. For this example, use scopolamine hydrochloride. The injection process is controlled using software which provides various options. For example, use the step function to define injection volume, and press the injection button every minute according to the clock of the recording software. Note: The exact duration of the injection block is substance and experiment dependent, e.g. for scopolamine use $2 \mathrm{~nL}$ injections each minute for 10 minutes $(20 \mathrm{~nL}$ in total). It is preferable not to advance the electrodes and micropipette during the injection block.

2. Note the time and the trial during which the substance is injected, the depth of the electrodes and micropipette, as well as the amount of ejected substance.

3. Follow the injection block with a recovery block, in which no substance is injected. The duration of the recovery block is substance specific and needs to be defined in pre-tests. Monitor and maintain the recording quality of the selected single units until the end of the recovery block.

4. Repeat the three blocks for as long as the recording quality and motivation of the monkeyallow.

\section{Post recording procedures}

1. After data recording, retract electrodes and micropipette into the guide tubes and then manually retract the guide tubes. Remove the recording system from the recording chamber of the monkey. Release the syringe from the injection pump and transfer the system to the preparation area for cleaning.

2. Handle the animal (including cleaning of the recording chamber ${ }^{18}$ ) according to the standard procedures of the laboratory and return it to the housing facility.

3. Rinse the outside of the guide tubes with hydrogen peroxide (3\%) and then with deionized water. Drive electrodes and micropipette out of the guide tubes, rinse with hydrogen peroxide and then deionized water.

4. Exchange the barrel of the syringe with a barrel of a syringe filled with sterile saline, keeping the needle in the tube. Flush the tube and the micropipette with 1-2 $\mathrm{mL}$ of saline. After flushing, remove the barrel and fill it with air. Reinsert the barrel into the needle and dry the tube and the micropipette from the inside by gently pushing air through.

5. Store the guide tubes, extended electrodes and micropipette immersed in the enzyme solution to avoid drying as well as to ensure the breakdown of organic material.

\section{Post recording procedures}

1. After data recording, retract electrodes and micropipette into the guide tubes and then manually retract the guide tubes. Remove the recording system from the recording chamber of the monkey. Release the syringe from the injection pump and transfer the system to the preparation area for cleaning.

2. Handle the animal (including cleaning of the recording chamber ${ }^{18}$ ) according to the standard procedures of the laboratory and return it to the housing facility.

3. Rinse the outside of the guide tubes with hydrogen peroxide (3\%) and then with deionized water. Drive electrodes and micropipette out of the guide tubes, rinse with hydrogen peroxide and then deionized water.

4. Exchange the barrel of the syringe with a barrel of a syringe filled with sterile saline, keeping the needle in the tube. Flush the tube and the micropipette with 1-2 $\mathrm{mL}$ of saline. After flushing, remove the barrel and fill it with air. Reinsert the barrel into the needle and dry the tube and the micropipette from the inside by gently pushing air through.

5. Store the guide tubes, extended electrodes and micropipette immersed in the enzyme solution to avoid drying as well as to ensure the breakdown of organic material. 


\section{Representative Results}

Figure 2 depicts the spatial attention task the monkey performed while the injection process was conducted. The monkey was trained to attend to either the stimulus located within the receptive field of the recorded neuron (attend-in), the stimulus located outside of the receptive field (attend- out) or the fixation point (attend-fix). These conditions allow a comparison of neuronal activity in different attentional states.

Figure 3 shows a peri-stimulus time histogram of a sample neuron in an experiment using scopolamine, a muscarinic cholinergic antagonist. The plot demonstrates the response suppression during scopolamine injection versus no injection, when a pattern moving in the cell's preferred direction is presented inside the neuron's receptive field and is attended by the animal. The two first peaks represent the neuron's response

to the on- and offset of the spatial cue, which appears inside its receptive field. This is followed by the response to the moving pattern which appears on the screen $500 \mathrm{~ms}$ after cue onset. The gray shaded area depicts the analysis period used to calculate the average firing rate for every trial. The green area highlights the suppressive influence of scopolamine injection on the cell's firing rate. The dark green region shows the suppression within the analysis period.

Figure 4a shows the effect of scopolamine on the average firing rate of the sample neuron in each of the three attentional conditions. The neuron's firing rate for the two spatial attention conditions (attention inside or outside of the receptive field of the recording neuron) as well asfor the sensory condition (attention at fixation point) dropped shortly after the first injection of the injection block (grey shaded area) and during the recovery block increased after a delay to the same level as before the injection.

Figure $4 \mathrm{~b}$ shows a control recording from a second sample neuron in which saline $(0.9 \% \mathrm{NaCl})$ was injected, using the same protocol as forthe scopolamine injection. During the injection block no change in the neuron's firing rate was observed compared to the control block.

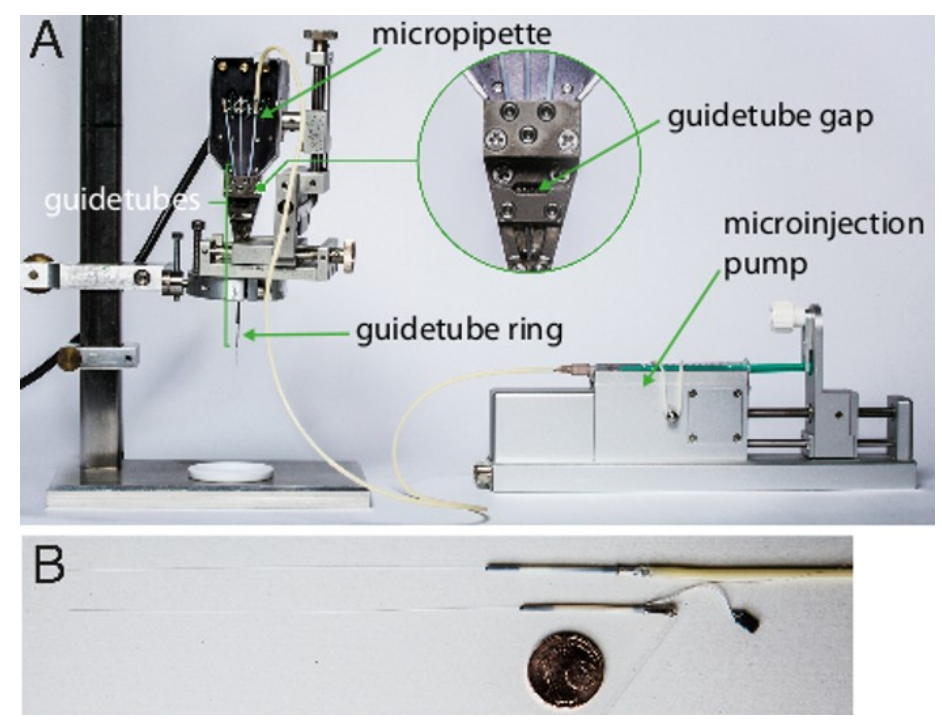

Figure 1. Set-up used for pharmacological manipulation while recording. (A) Depicts the microinjection pump and

the electrophysiological recording system equipped with electrodes and micropipette. The guidetube gap, into which silicon oil is inserted to lubricate the electrodes and micropipette, is shown enlarged. (B) Displays an example micropipette (above) and recording electrode (below). For size comparison, a euro cent (diameter: $16 \mathrm{~mm}$ ) is placed underneath. 


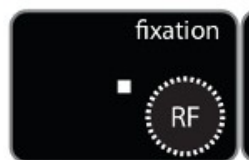

$150 \mathrm{~ms}$

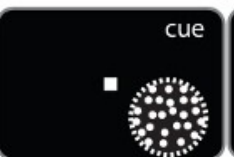

$150 \mathrm{~ms}$

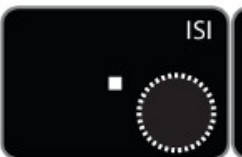

$350 \mathrm{~ms}$

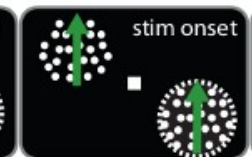

$200-2500 \mathrm{~ms}$

after stimulus onset

Figure 2. Task design to guide spatial attention. Monkeys were trained to detect a motion direction change in the cued dot pattern. The cue was either placed within the neuron's receptive field (attend-in), as shown in the figure, or outside of it (attend-out). As a sensory control, the monkey was trained to detect a luminance change of the fixation point (attend-fix)

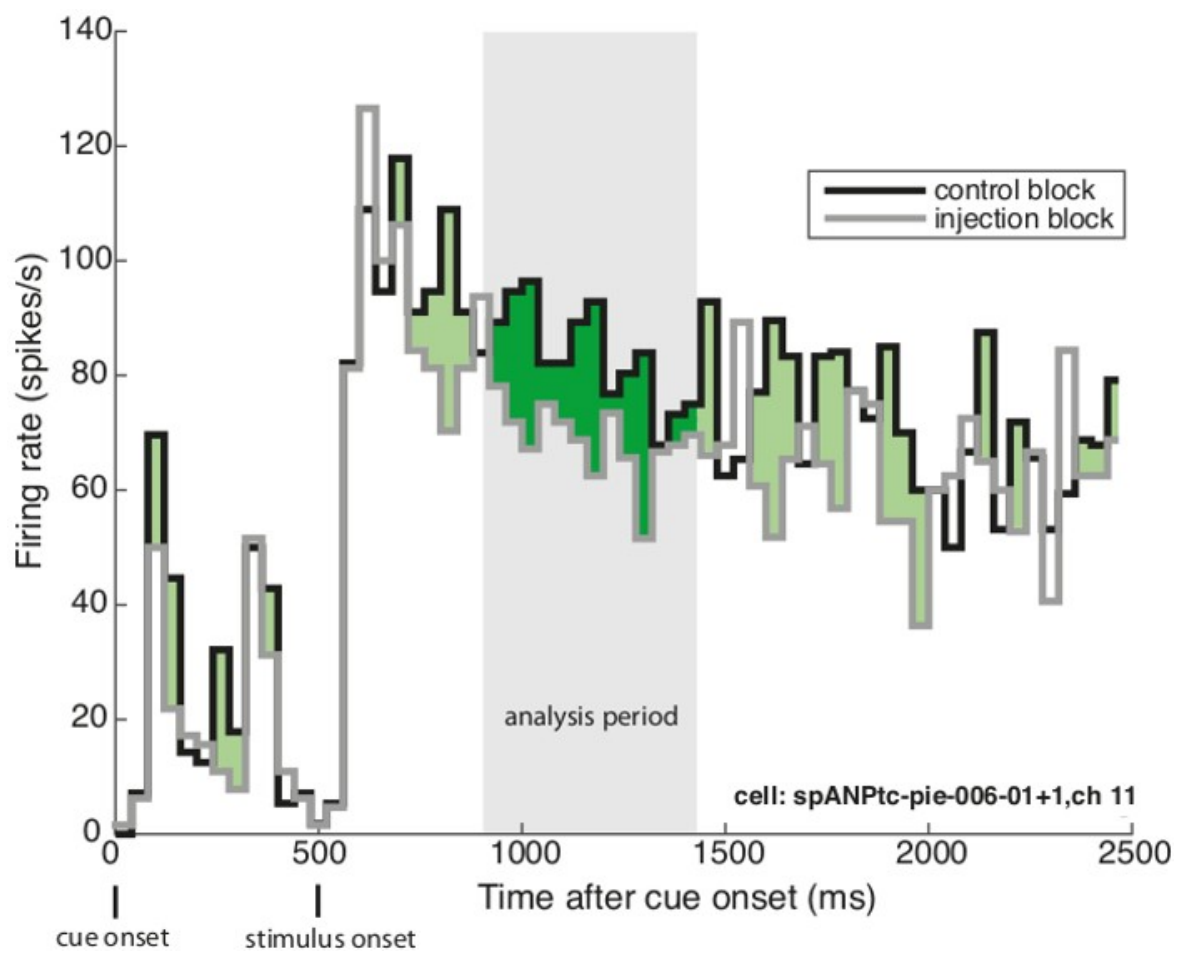

Figure 3. Influence of antagonist scopolamine on firing rate. The peri-stimulus time histogram for a sample neuron is shown for the attend- in condition (attention inside the receptive field of the recorded neuron) during the injection block and during the control block. The x-axis depicts the time in milliseconds after cue onset and the $y$ axis shows the firing rate in spikes/sec. The grey area depicts the analysis period (300-800ms after stimulus onset) used to calculate the trial-averaged firing rate. The green shaded area shows the suppression in firing rate across the two conditions. The dark green color highlights the suppression within the analysis period 

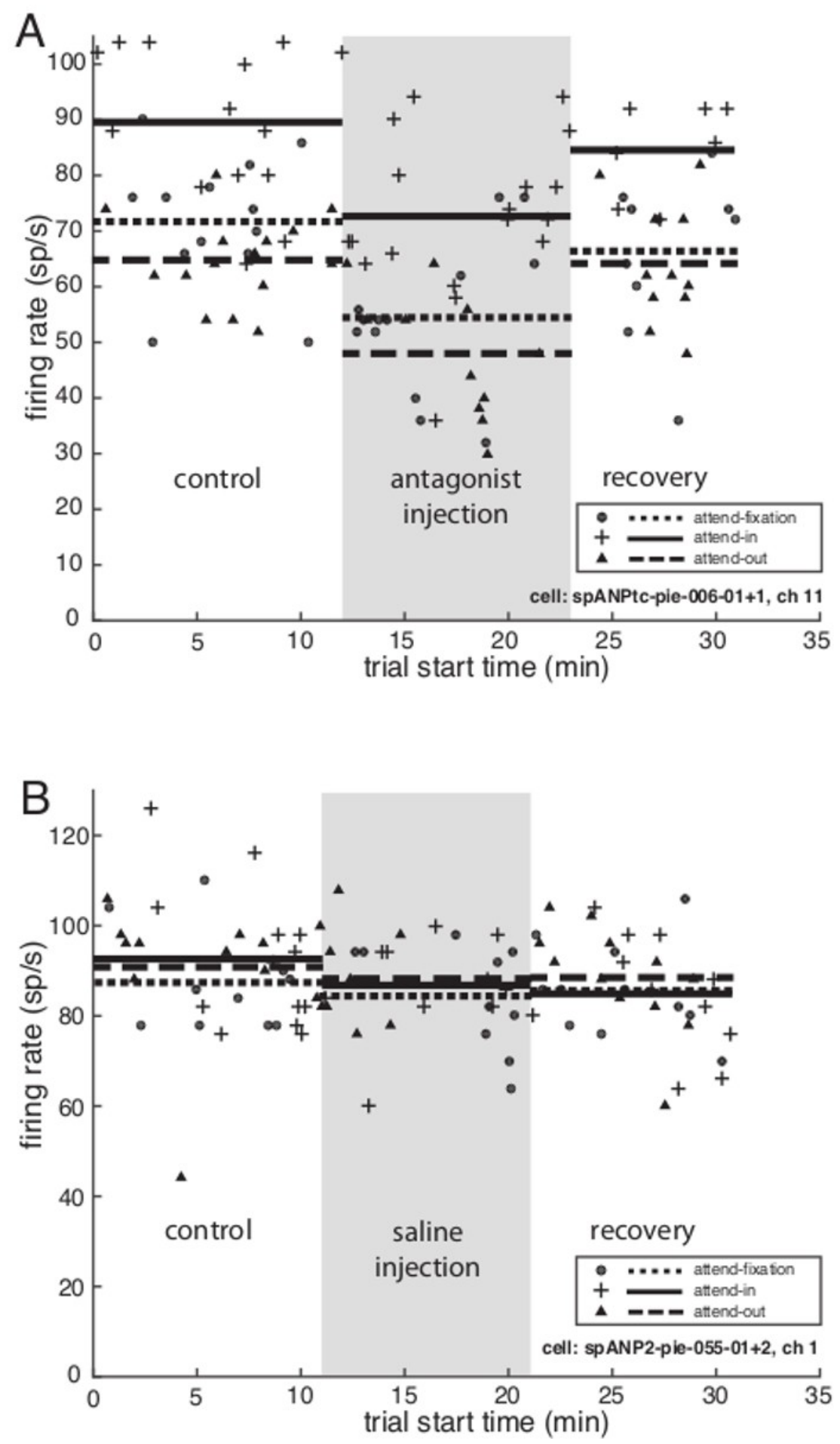

Figure 4. Effect of scopolamine and saline on firing rate. (A) Antagonist scopolamine injection. The trial-averaged firing rate of the sample cell from Figure 3 over the course of the experiment is shown for the preferred stimulus for all three attentional conditions. The $\mathrm{x}$-axis depicts the trial start time in minutes and the $y$-axis shows the unit's firing rate in spikes per seconds. Symbols ${ }^{+}$attend-in, ${ }^{\bullet}$ attend-fix, $\boldsymbol{\Delta}$ attend-out) represent the neuron's firing rate within the analysis period in every successfully performed trial, and horizontal lines (solid line: attend-in, dotted line: attend-fix, dashed line: attend-out) show average firing rate for the three different experimental blocks (control, injection, recovery). The grey shaded area shows the injection block, beginning with the first injection and ending one minute after the last injection. During the injection block $2 \mathrm{~nL}$ of 0.1 molar scopolamine were injected every minute with an injection velocity of $2 \mathrm{~nL} / \mathrm{s}$. (B) Saline injection. The firing rate of a sample cell over the course of the control experiment is shown for the preferred stimulus for all three attentional conditions. Grey shaded area visualizes the block of saline injection. 


\section{Discussion}

Here we have illustrated in detail how to perform reliable and precise injections and high quality single-cell recordings with an "off-the-shelf" pressure injection system. While this method of drug delivery has previously been used in behaving monkeys (reviewed in ${ }^{17}$ ), the system presented here has advantages, reviewed below.

As illustrated in Figure 4a, the system described here can provide stable measurement of single neuron activity with and withoutpharmacological injections in the direct vicinity of the recording site. As shown in Figure 4b, the injection of a control substance, saline, did not lead to a change

in firing rate. This control demonstrates that the injection process itself has no measurable influence on the firing properties of the recorded neurons.

The spatial configuration of neuron, recording electrode, and micropipette is of crucial importance in these experiments. Although a precise measurement of their relative positions in the tissue during recording is not possible, we can consider and control for possible sources of variance. First, during volume injection there is a risk that the neuron of interest may be displaced away from the recording electrode, affecting the stability of recorded signals. For that reason it is prudent to compare the firing rate before and after the injection block to verify signal stability. Second, the guide tube configuration of the recording system defines the distance between electrodes and micropipette (e.g. $305 \mu \mathrm{m}$ in the concentric 3-channel system used in this experiment). As the system provides precise position control for the depth of electrodes and micropipette in the tissue, the distance between them can be minimized by carefully calibrating relative depth before recording (step 3.5), and keeping them at a common depth during recordings.

\section{Potential limitations}

In addition to in-house quality control by the manufacturer, the system needs to be validated under lab conditions, as different brands of tubes, syringes etc. can be used and could lead to differences in ejected volumes. Although the system can be used to inject very small volumes as in the experiment shown here, these are below the minimum volume that can be validated due to practical measurement limits in a normal laboratory environment. However, larger injection volumes can be used to infer the relation between the software-defined volume and the volume ejected by the hardware. If transparent tubes are used, an additional visual check of the injection process is possible by measuring the displacement of a visual marker.

Inserting the micropipette into the system is more demanding than electrode insertion, as the diameter of the micropipette is slightly larger and the material is more fragile. In addition, joining the tube to the pin of the micropipette is challenging as it entails a high risk of breaking the upper part of the micropipette. However, the lifetime of a successfully loaded micropipette is several months, even with daily use.

In practice, we have not yet encountered a blockage in the injection system during post-recording cleaning of the system. Nevertheless, no "online" check is possible, and there is a risk that a physical blockage (such as tissue at the micropipette tip) might prevent substance injection. It might therefore be advisable to analyze the data conservatively, such as including only those cells in further analysis that show significant changes in firing rates between control and injection blocks of the experiment.

Despite their small diameter, microelectrodes and pipettes will displace brain tissue and may cause some local tissue damage. This can be minimized by manually positioning the tip of the guide tubes just above the dura mater. The electrodes then penetrate the dura and their intactness is inferred by measuring their impedances online. Afterwards, the micropipette is inserted. When using this approach, regular removal of tissue above the dura is recommended to further reduce the risk of electrode or pipette breakage.

\section{Comparison to alternative methods}

The system used here shows clear advantages compared to other pressure injection systems. One strong advantage is the diameter of the micropipette (approximately $100 \mu \mathrm{m}$ ), which is half the size of other available probes $^{17}$ and therefore minimizes neural tissue damage. In contrast to previous designs, the current system employs spatially separated recording electrode and micropipette. Although other systems provide a smaller distance between electrode and pipette, the system described here allows independent depth changes of electrodes and pipette, thus permitting variable relative distances within a recording session. Importantly, no compromise regarding recording quality needs to be made,

as the injection system is an extension of an established recording device. While only one micropipette and thus one substance is used in this protocol, it is possible to inject several substances within one experimental procedure. To achieve this, several micropipettes can be threaded into separate guide tubes and connected to syringes mounted in individual injection pumps. Finally, controlling the system is easy, as only one computer program is needed to advance the electrodes and micropipette, and to perform the pressure injection during the experiment.

Comparing pressure injection to iontophoresis, there are relative advantages and disadvantages. For example, pressure injection requires greater volumes to be introduced into the tissue than iontophoresis, thus increasing the risk of neuronal displacement. The current protocol used volumes in the $\mathrm{nL}$ range, and we rarely experienced noticeable changes in a recorded cell's signal quality. The system also allows larger volumes to be injected, which is potentially useful for behavioral manipulations but could impact stability of neuronal recording. A clearadvantage of pressure injection over iontophoresis is the larger variety of useable substances as there is no requirement to use charged substances. 
However, $\mathrm{pH}$ values should be checked and compared between experimental and control substances (e.g. saline).

The question might arise why to use the long-established method of pressure injection instead of newer techniques such as optogenetics for manipulating neural activity. Although well established in rodents, optogenetics is not yet reliably established in rhesus monkeys. In particular, it does not yet allow the local manipulation of cells selective for a particular neurotransmitter type. In the longer run, we see great potential for the combination of the advantages of pharmacological manipulations with the advantages of optogentic manipulations in elucidating the neural basis of cognitive functions.

Here we have shown how pressure injection can be used to pharmacologically manipulate a locally restricted area in the brain of awake, behaving rhesus monkeys. We hope that this method inspires other scientists to investigate neuromodulatory contributions to the dynamics of neuronal activity. 


\section{Disclosures}

The authors have nothing to disclose.

\section{Acknowledgements}

This work was supported by grants of the Deutsche Forschungsgemeinschaft through the Collaborative Research Center 889 "Cellular Mechanisms of Sensory Processing" to S.T. (Project C04). We thank Sina Plümer, Leonore Burchardt, Dirk Prüsse, Klaus Heisig and Ralf Brockhausen for technical and animal-related support and our collaborators in the Stem Cell Unit of the German Primate Center, Dr. Katharina Debowski and Anna Magerhans, for technical assistance in the filtration process.

\section{References}

1. Noudoost, B., Moore, T. The role of neuromodulators in selective attention. Trends Cogn Sci. 15(12), 585-591 (2011).

2. Jochems, A., Reboreda, A., Hasselmo, M., Yoshida, M. Cholinergic receptor activation supports persistent firing in layer III neurons in the medial entorhinal cortex. Behav Brain Res. 254,108-115 (2013).

3. Thiele, A., Herrero, J.L., Distler, C., Hoffmann, K.P. Contribution of cholinergic and GABAergic mechanisms to direction tuning, discriminability, response reliability, and neuronal rate correlations in macaque middle temporal area. J Neurosci. 32(47):16602-16615 (2012).

4. Thienel, R., et al. Muscarinic antagonist effects on executive control of attention. Int J Neuropsychopharmacol. 12(10), 1307-1317(2009).

5. Anthony, B.L., Dennison, R.L., Aronstam, R.S. Disruption of muscarinic receptor-G protein coupling is a general property of liquid volatile anesthetics. Neurosci Lett. 99(1-2), 191-196 (1989).

6. Yamakura, T., Bertaccini, E., Trudell, J.R., Harris, R.A. Anesthetics and ion channels: molecular models and sites of action. Annu Rev Pharmacol Toxicol. 41, 23-51 (2001).

7. Herr, N.R., Wightman, R.M. Improved techniques for examining rapid dopamine signaling with iontophoresis. Front Biosci. 5, 249-257 (2013).

8. Bevan, P., Bradshaw, C.M., Pun, R.Y., Slater, N.T., Szabadi, E. The relative contribution of iontophoresis and electro-osmosis to the electrophoretic release of noradrenaline from multi barrelled micropipettes [proceedings]. Br J Pharmacol. 67(3), 478-479 (1979).

9. Herr, N.R., Kile, B.M., Carelli, R.M., Wightman, R.M. Electroosmotic flow and its contribution to iontophoretic delivery. Anal Chem. 80, 8635-8641 (2008).

10. Thiele, A., Delicato, L.S., Roberts, M.J., Gieselmann, M.A. A novel electrode-pipette design for simultaneous recording of extracellularspikes and iontophoretic drug application in awake behaving monkeys. $J$ Neurosci Meth. 158(2-4), 207-211 (2006).

11. Lalley, P.M. Microiontophoresis and Pressure Ejection: Modern Techniques in Neuroscience (eds. Windhorst U., \& Johansson H.) 193-209, (1999).

12. Malpeli, J.G., Schiller, P.H. A method of reversible inactivation of small regions of brain tissue. J Neurosci Meth. 1(2), 145-59(1979).

13. Malpeli, J.G. Reversible inactivation of subcortical sites by drug injection. J Neurosci Meth. 86(2), 119-28 (1999).

14. Dias, E.C., Segraves, M.A. A pressure system for the microinjection of substances into the brain of awake monkeys. J Neurosci Meth. 72 (1), 43-47 (1997).

15. Szente, M.B., Baranyi, A., Woody, C.D. Effects of protein kinase $\mathrm{C}$ inhibitor H-7on membrane properties and synaptic responses of neocortical neurons of awake cats. Brain Res. 506 (2), 281-286 (1990).

16. Woody, C.D., Bartfai, T., Gruen, E., Nairn, A. Intracellular injection of cGMP-dependent protein kinase results in increased input resistance in neurons of the mammalian motor cortex. Brain Res. 386 (1-2), 379-385 (1986).

17. Noudoost, B., Moore, T. A reliable microinjectrode system for use in behaving monkeys. J Neurosci Meth. 194 (2), 218-23 (2011).

18. Association of Primate Veterinarians. Cranial Implant Care Guidelines for Nonhuman Primates in Biomedical Research, http://

www.primatevets.org/Content/files/Public/education/Cranial\%20Implant\%20Care\%20Guidelines.pdf (2015).

19. Treue, S., Martinez-Trujillo, J.C. Feature-based attention influences motion processing gain in macaque visual cortex. Nature. 399, 575-579 (1999).

20. Martinez-Trujillo, J.C., Treue, S. Feature-based attention increases the selectivity of population responses in primate visual cortex. Curr Biol.

14 (9), 744-751 (2004). 


\section{The role of the cholinergic system in attentional modulation in area MT of}

\section{the primate visual cortex}

Vera Katharina Veith ${ }^{1}$, Cliodhna Quigley ${ }^{1}$, Stefan Treue ${ }^{1,2}$

${ }^{1}$ Cognitive Neuroscience Laboratory, German Primate Center, Goettingen, Germany

${ }^{2}$ Bernstein Center for Computational Neuroscience, Goettingen, Germany

\section{Abstract}

Attentional modulation of sensory responses in extrastriate visual cortex of primates is characterized by gain changes, which are multiplicative changes of firing rates for a given combination of stimuli in the receptive field and the attentional state of the animal. There is evidence that the cholinergic system may be responsible for such changes. In this study, we aim to directly link attention-induced changes in neuronal response in macaque area MT to local cholinergic activity. While the monkey performed a task requiring top-down spatial attention, we induced local neuropharmacological manipulations targeting the cholinergic system while recording single cell activity. Local enhancement of the neuromodulator acetylcholine led to increased attentional modulation. A blockage of the muscarinic receptor type showed a significant 
increase in attentional modulation, an effect opposite to that found in area V1 (Herrero et al., 2008). These results suggest a visual area dependent difference in the mechanistic role of the cholinergic system in visual attention processes.

\section{Introduction}

Attention induces an increased representation of attended objects on a neuronal level (Treue and Maunsell, 1996). This effect was reported to be very consistent across studies investigating different visual areas (Roelfsema et al., 1998; Reynolds et al., 1999). However, the exact neurotransmitter contribution triggering this effect is to a big extent ambiguous. There is ample evidence of cholinergic participation in attentional modulation, primarily gained in rodent studies (Sarter et al., 2005; Parikh and Sarter, 2008; Fournier, 2004). Additionally, cognitive deficits characterizing Dementia and Alzheimer's disease were ascribed to cholinergic cell loss in the basal forebrain, the main cholinergic source for the cortex, in human (Wevers et al., 2000; McGaughy et al., 2002). However, these studies often lack a link between the fast effects of attentional modulation and local cortical cholinergic release, as the studies either measure global cholinergic effects using fMRI (Kumari et al., 2003) or have a slow temporal resolution using microdialysis (Arnold et al., 2002). Consequently, the precise nature of the cholinergic contribution to attentional modulation is still ambiguous.

A study performed in macaque visual area V1 was the first to shed light on the underlying cellular mechanisms of the well-discovered effects of spatial attention on firing rates in an early visual area (Herrero et al., 2008). Here a cholinergic agonist or antagonist was iontophoretically injected in the direct vicinity of the recording electrode while the macaque monkey performed a spatial attention task. A correlation was found between a moderate increase of 
acetylcholine and an increase in attentional modulation. In addition, the authors found muscarinic, but not nicotinic, receptor contribution in attentional modulation in V1 of macaque monkey.

However, it has also been shown that visual area $\mathrm{V}_{1}$ has specific anatomical characteristics that distinguish it from other visual areas, such as a reduced amount of inhibitory neurons as well as an altered quantity of cholinergic receptor subtypes (DeFelipe et al., 1999, Disney et al., 2006). This suggests a diverse role for cholinergic contribution, and therefore area V1 is of limited suitability to serve as a model for cholinergic modulation of visual cortical circuits in the macaque.

In this study we recorded neuronal activity in visual area MT, a well understood mid-level visual area in terms of its sensory properties and its attentional modulation characteristics (Treue and Maunsell, 1996). We recorded from single cells in two awake, behaving rhesus monkeys while they performed a spatial attention task. We compared firing rates when the stimulus in the receptive field was attended vs. unattended to quantify gain changes by spatial attention. During recordings, we used pressure injection to pharmacologically manipulate the direct vicinity of the recorded neuron in a block-wise fashion (Veith et al., 2016). We used the antagonist scopolamine or mecamylamine to block the muscarinic or nicotinic cholinergic receptor subtype respectively, or the agonist acetylcholine to increase its extracellular concentration. The pattern of attentional modulation during injection was compared to baseline blocks recorded before injection.

The aim of this study is to investigate whether there is an interactive effect of spatial attention and neuropharmacological manipulation on neuronal response in area MT in the macaque monkey, thereby shedding light on local cholinergic mechanism of attention. 


\section{Material and Methods}

\section{Monkey surgery}

Two male macaque monkeys (macaca mulatta) were implanted with custommade titanium head holders to circumvent movement during the recording procedure. Additionally, they were implanted with a recording chamber (Crist Instruments, Hagerstown, MD, USA or 3DI, Jena, Germany) over the left or right hemisphere. The position of the recording chamber was planned based on anatomical fMRI scans using a MATLAB-based (TheMathworks, Inc.) software (Ohayon and Tsao, 2012). All surgeries were performed aseptically under balanced anesthesia using Sevofluran, Propofol and Remifentanil.

\section{Apparatus}

During recordings, monkeys were seated in a custom-made primate chair in a dark cabin with a distance of $57 \mathrm{~cm}$ from the computer monitor (Quato Display $240 \mathrm{~m}$ ). Visual stimuli were presented with a refresh rate of $60 \mathrm{~Hz}$ and a resolution of 1920 x 1200 pixel. Eye position was recorded using an eyetracker (ET49, Thomas Recording, Giessen, Germany) with a frame rate of $230 \mathrm{~Hz}$. Acute recording/injection was performed using a multielectrode manipulator equipped with a pressure injection system (MiniMatrix, Thomas Recording, Giessen, Germany). Details about preparation, handling and care of this system can be gleaned from the prior methods paper (Veith et al., 2016). Recording of neuronal signals and real-time spike sorting was performed with a data acquisition system (MAP, Plexon Inc., Dallas, USA). All stimuli were generated and presented using custom-made software built for real-time visual experiments running on an Apple Macintosh PowerPC. In addition, the software 
monitored the eye position, controlled fluid reward release and collected behavioral as well as electrophysiological data.

\section{Stimuli}

Used stimuli were white random dot patterns (RDP) presented on a uniform grey background (luminance $27 \mathrm{~cd} / \mathrm{m}^{2}$ ). Each RDP consists of small bright dots (luminance: $38 \mathrm{~cd} / \mathrm{m}^{2}$, size $0.1 \mathrm{deg}$ ), coherently moving linearly within a circular, stationary aperture. One RDP was placed at the most responsive part of the receptive field (RF), the second RDP was placed opposite the RF in the other hemifield with the same relative distance to the centrally presented fixation point. The speed of the moving dots was adjusted to the recorded cell's preferences, but always lay between 4 and $12 \mathrm{deg} / \mathrm{sec}$. Preferred movement direction of the recorded cell was defined based on results gained in previously performed tuning sessions. The radius of the dot pattern was increased when stimuli had to be placed very eccentrically in order to stay detectable for the monkey (range of $2-3 \mathrm{deg}$ ).

\section{Substances}

In order to manipulate the cholinergic system, we used the general agonist acetylcholine, the muscarinic antagonist scopolamine, and the nicotinic antagonist mecamylamine in various concentrations and volumes (SigmaAldrich, St.Louis, Missouri, USA). All substances were diluted in saline $(\mathrm{NaCl}$, BBraun, Melsungen, Germany). For acetylcholine, concentrations of 0.1, 0.15 and $0.2 \mathrm{~mol} / \mathrm{l}$ were used and volumes of 2,3 , and $4 \mathrm{nl} /$ minute were injected. Scopolamine was injected with concentrations of $0.01,0.05$ and $0.1 \mathrm{~mol} / \mathrm{l}$ and volumes of 1, 2, and $4 \mathrm{nl} /$ minute. Mecamylamine was used in a concentration of $0.1 \mathrm{~mol} / \mathrm{l}$ and a volume of $6 \mathrm{nl} /$ minute. For a control, saline was injected with a 
volume of 2, 4, and $6 \mathrm{nl} /$ minute in order to mimic the influence of different volumes onto the recorded neuron. Supplementary figure 2 shows the concentration and volume combinations used in this study. Measured $\mathrm{pH}$ values stayed at approx. 5 for all substances used. Different concentrations of the substances used had only weak influences on $\mathrm{pH}$ values.

\section{Behavioral task}

This task was designed to guide the monkeys' spatial attention to various locations on the monitor. Therefore the two monkeys were trained to detect a motion direction change in the cued dot pattern. The cue was either placed within the neuron's receptive field (attend-in) or outside of it in the other hemifield (attend-out). As a sensory condition the monkeys had to detect a slight luminance change in the fixation point (attend-fix).

Monkeys initiated every trial by holding a lever and fixating the centrally presented fixation point. In the attend-in and attend-out conditions, the centrally presented fixation point remained red (square with side length: 0.16 deg; luminance: $14 \mathrm{~cd} / \mathrm{m}^{2}$ ) during the entire trial. After a delay of $150 \mathrm{~ms}$ a static dot pattern, serving as an exogenous cue, was presented for $150 \mathrm{~ms}$ at the future stimulus position either within the neuron's receptive field (attend-in) or outside of it (attend-out). An inter-stimulus interval of $350 \mathrm{~ms}$ followed, where only the fixation point was shown. Subsequently, two RDPs were presented on the screen, one placed within the neuron's receptive field, the other in the opposite hemifield. They both moved linearly in the same direction, either in the preferred direction of the recorded neuron or in its null direction (+18o deg). Monkeys had to respond to a slight direction change (duration $130 \mathrm{~ms}$ ) at the cued location (target) and had to ignore a direction change at the uncued location (distractor). The angle of direction change varied from 25 to $35 \mathrm{deg}$, depending on the stimulus position. The aim was to adjust the task difficulty 
depending on stimulus position, choosing bigger angles for more eccentric stimuli. The direction change could occur in a time window of $200-2500 \mathrm{~ms}$ after stimulus onset. In $1 / 10^{\text {th }}$ of the trials no direction change happened at the target position and monkeys were rewarded for not responding until the trial ended.

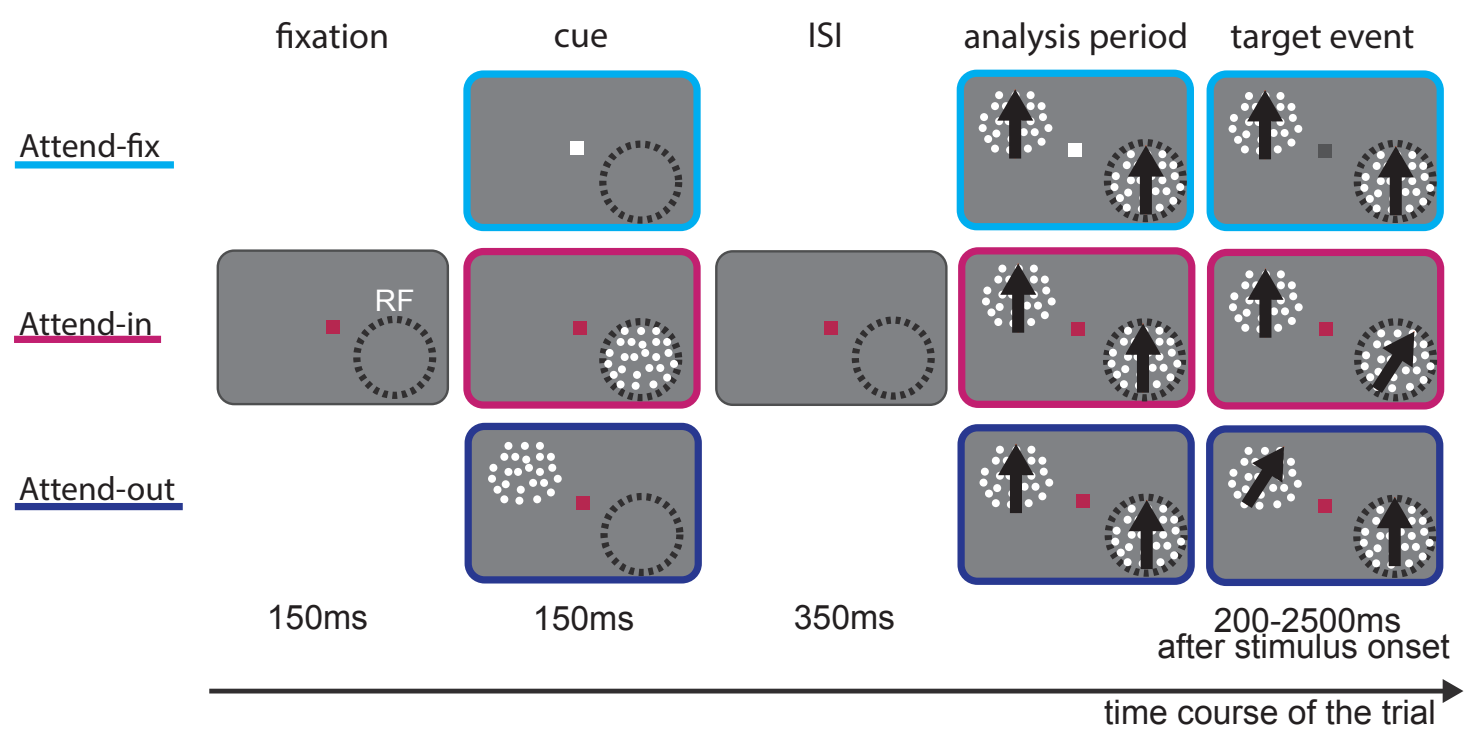

Figure 1. Schematic trial structure of the spatial attention task. The three attention conditions, attend-fix, attend-in, and attend-out, were shown in a random order. In the attend-fix trials the monkey had to respond to a luminance change of the centrally presented fixation point. In attend-in and attend-out trials, the monkey had to respond to a direction change of the cued dot pattern. (ISI $=$ inter stimulus interval).

In the sensory condition (attend-fix) the monkeys had to respond to a slight luminance change of the fixation point and had to ignore direction changes of the two shown RDPs. Here, the fixation point changed from red to gray color immediately after the monkeys started the trial. The behaviorally relevant luminance change (from 85 to $52 \mathrm{~cd} / \mathrm{m}^{2}$ ) of the fixation-point could occur in a time-window of 200-2500ms. Additionally, we had a baseline condition, where only the fixation point was shown on the screen and the monkeys had to detect a luminance change of it. 
In all conditions, monkeys had to respond by releasing a lever within a time window of $550 \mathrm{~ms}$ after target onset and were rewarded with a drop of juice. Trials of all attentional and sensory conditions were presented in a random fashion. Each trial was aborted when eye fixation was interrupted or eye gaze moved outside of the fixation window (1.2 deg radius around the fixation spot).

\section{Experimental procedure}

At the beginning of a recording session, guide tubes were manually lowered to a position in the recording chamber at which they just penetrated the dura with their tip. Subsequently the electrodes were mechanically ejected into the cortex while simultaneously monitoring the impedances to avoid physical damage to tissue and electrodes. When electrodes were successfully lowered, the micropipette was ejected to the same level. From there on, all electrodes and the micropipette were driven with the same speed to the same depth. More details can be found in the previous methods paper (chapter 2.3).

Single unit activity was recorded using two single tungsten electrodes of two different impedances (0.2-0.5 $\mathrm{M} \Omega$ or 1-2 $\mathrm{M} \Omega$ ) placed in a multielectrode recording system (Thomas Recording, Giessen, Germany). Data were filtered $(150 \mathrm{~Hz}-5 \mathrm{kHz})$ and amplified (gain range 1000-32000). Isolation of single units was performed using online window discrimination (RASPUTIN, Plexon Inc., Dallas, Texas, USA).

\section{Characterization of isolated cells}

Based on their response properties, isolated cells were ascribed to area MT, the target cortical area in this study. Therefore a mapping and a tuning experiment were conducted at the start of a recording session. In the mapping experiment, 
the RF size was determined as well as its hotspot, where visual stimulation elicited the highest response. This was done by manually moving a static dot pattern on the monitor. When the defined RF size matched the size of an average MT cell (diameter approx. $5 \mathrm{deg}$ ) we continued with the tuning task. Here one stimulus (RDP) was placed at the previously defined hotspot. While the monkeys performed a luminance detection task, where a slight luminance change of the centrally presented fixation point had to be detected, the dots of the RDP performed a coherent linear movement to one out of 12 direction in steps of $30 \mathrm{deg}$ with various speeds $(2,4,8$, and $12 \mathrm{deg} / \mathrm{s})$. Each trial had a sequence of randomly chosen speed and direction combinations of a single pair of opposing directions $(0 / 180 ; 30 / 210$; etc.). Based on the tuning profile of the neuron the preferred direction and speed were defined for the subsequent attentional task. When recording several units, the stimulus properties were chosen to activate all neurons.

\section{Main experiment with pharmacological manipulations}

With the information gained in the mapping and tuning tasks, we generated the main experimental task with stimulus properties targeting the isolated neuron(s).

The main experiment is subdivided into three blocks: control, injection, and recovery (see Figure 2). 


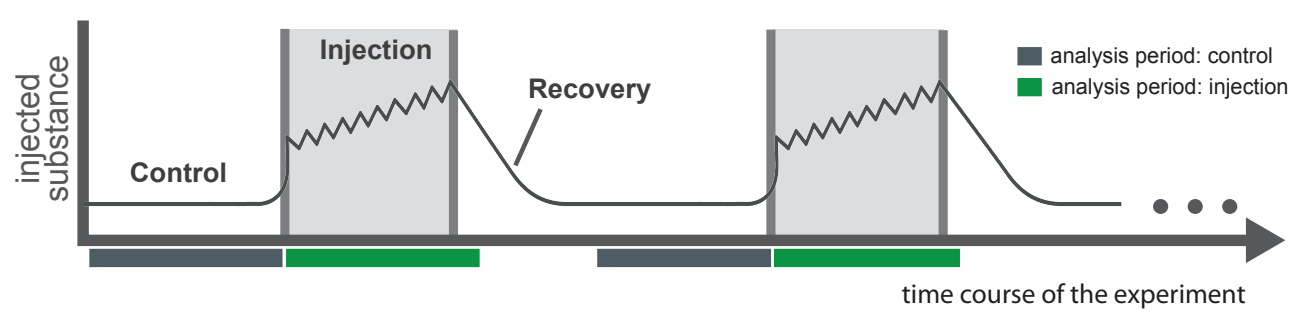

Figure 2. Experimental design. One full cycle of the main experiment consists of control, injection, and recovery blocks. The cycle can be performed several times during one experiment. The exact definition of the three blocks is described in the text. The grey horizontal bar depicts the analysis period for the control condition. The green horizontal bar depicts the analysis period for the injection.

During all blocks, the monkeys preformed all attentional task conditions in a random fashion. The injection block was started after sufficiently many hit trials in the initial control block (at least 11 repetitions of each conditions). During the control condition, neuronal activity was measured in the absence of pharmacological influence. In the subsequent injection block a specific amount of a substance was injected every minute, or twice a minute, using pressure injection. In total, 3 different substances were used in this study: acetylcholine (general agonist), scopolamine (muscarinic cholinergic antagonist) and mecamylamine (nicotinic cholinergic antagonist). As a control substance saline was injected. Supplementary Figure 2 depicts all concentrations and volumes used in this study.

A recovery block followed the injection block, where no substance was injected. In this block the neurons' activity should recover, if affected during the injection block, to the same value as in the control period. In most of the experiments one full cycle, covering control, injection and recovery block, were performed. In other rare cases an additional or even a third cycle of pharmacological manipulation was recorded. 


\section{Data analysis}

With our custom-made software we were able to analyze the behavioral data as well as the spike train of the isolated cells online during the recording session. This helped us gain an initial impression of the data quality while recording. Final data analysis was performed offline using custom scripts written in MATLAB (The MathWorks, Natick, MA).

For the main analysis, only the response to the preferred direction of each neuron was used. Firing-rates were averaged in an analysis window of 300-800 ms after stimulus onset for every trial.

We compared firing rates in the absence (control block) and presence of a drug (injection block). The analysis period for the control block started from the beginning of the data file until the first injection was performed. Control blocks for second and third injections are defined as periods immediately preceding start trial of injection (no shift in time) with duration equal to the duration of the corresponding injection period.

The analysis period for the injection block started one minute after the first injection and ended 2 minutes after the last injection was performed (see Figure 2).

\section{Inclusion criteria}

The first three inclusion criteria examined firing characteristics independent of injection and attention influences (see suppl. Figure 1). Therefore only data files were included that contained enough trial repetitions (a minimum of 5 trials) for every task condition. Additionally, recorded cells had to respond with certain strength to a stimulus on the screen, showing a minimum firing rate of 10 spikes/sec for the preferred direction condition in order to be included. A 
responsiveness check was also performed comparing the sensory conditions for preferred and null direction and fix-only during the control block. Cells were included in further analysis if they showed significant differences between the three fixation conditions (Kruskal-Wallis test, $\mathrm{p}<0.05$ ).

In the separate inclusion criterion applied to the substances with sufficiently many cells, a subset of cells was defined showing significant injection effects on firing rates in sensory conditions. These cells were therefore analyzed separately.

Of 128 well-isolated MT single units of two monkeys, 100 fulfilled the first three inclusion conditions when using the muscarinic antagonist scopolamine. 28 of 44 units were used for saline control. 30 out of 54 were used to analyze the effect of general agonist acetylcholine, and 5 out of 8 were used for nicotinic antagonist mecamylamine.

An injection modulation index (IMI) was used to quantify the influence of injection on firing-rates. It was defined as the difference in firing rate for two blocks, divided by the sum.

$$
I M I=\frac{R_{2}-R_{1}}{R_{2}+R_{1}}
$$

where $R_{1}$ is the respective firing rate in the control block and $R_{2}$ in the injection block. Therefore, a positive IMI indicated a response enhancement due to injection. The IMI was calculated separately for all attentional conditions: attend-fix, attend-in, attend-out.

In order to test for injection effects on attentional modulation, an attentional modulation index (AMI) was calculated and compared for control and injection blocks.

The AMI was used to measure the effect of attention on firing rate and was defined as: 


$$
A M I=\frac{Q_{2}-Q_{1}}{Q_{2}+Q_{1}}
$$

where $\mathrm{Q}_{1}$ is the respective firing rate in attend-out trials and $\mathrm{Q}_{2}$ in attend-in trials. Positive values indicate an increase in response by attention, whereas negative values denote suppression.

The AMI and the IMI were also used to define the percentage change (percAMI) in firing rate modulated by attention or injection.

$$
\operatorname{perc} A M I=\frac{2 x A M I}{1-A M I} \times 100 \%
$$

The percentage change for the IMI was calculated in the same manner as for the AMI.

Additionally, the difference in AMI or IMI between two conditions was calculated to compare the relative change in modulation and was therefore defined as:

$$
\Delta A M I=A M I_{2}-A M I_{1}
$$

$\mathrm{AMI}_{2}$ and $\mathrm{AMI}_{1}$ are respective attentional modulation indices for two experimental blocks, injection and control. Positive values indicate an increase in attentional modulation with injection, whereas negative values indicate suppression. 


\section{Behavioral results}

In addition, we were interested whether the local application of agonist or antagonist induced a behavioral change.

The monkeys were either cued to attend a spatial location inside the receptive field of the recorded neuron, outside of the receptive field in the other hemifield, or the centrally presented fixation point. The three attentional conditions were randomly shown on a trial-by-trial basis. We calculated and compared the reaction times for control and injection blocks in order to investigate the influence of the injected substances on the behavioral performance for all attentional conditions separately. Supplementary figure 3 illustrates the median reaction times for all attentional conditions for control and scopolamine injection for both monkeys separately.

Additionally, we correlated the change in median reaction time (injection minus control) with the corresponding injection modulation index, in order to directly link changes in neuronal response with behavior. We calculated a linear regression to statistically quantify the relationship between these two factors. 


\section{Results}

In order to investigate the neuronal spiking dynamics for every attentional condition, we calculated the peristimulus time histograms (PSTH) during the control block, where no substance was injected. As illustrated in figure 3, both monkeys show clear attentional enhancement of their firing rate, as the firing rates for attend-in condition are clearly elevated compared to firing rates in attend-out or attend-fix conditions (red vs. dark blue lines in figure 3). As both monkeys show a similar effect of spatial attention on their firing rate, we pooled the data from the two monkeys. Subsequent analysis is based on firing-rates in the time window of $300-800 \mathrm{~ms}$ after stimulus onset (grey shaded area in Figure PSTH_sco).
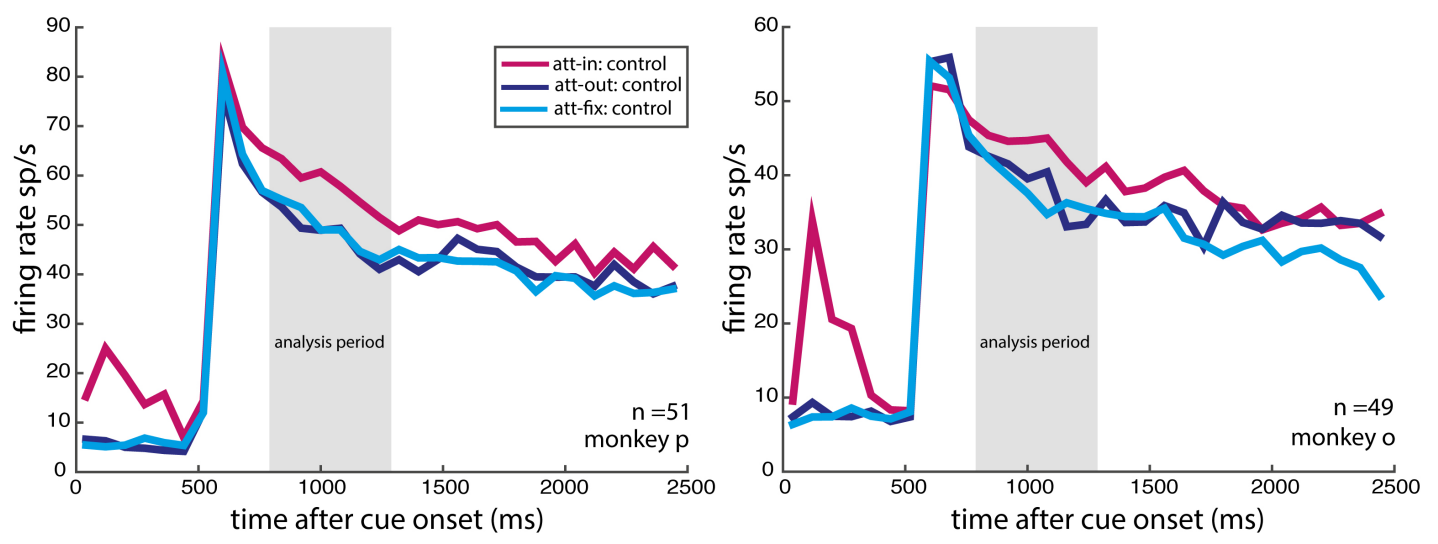

Figure 3. Effect of spatial attention on neuronal firing rate across trial time course. Peristimulus time histograms were calculated for the control condition for the scopolamine cell population of each monkey separately. The analysis period is defined as $300-800 \mathrm{~ms}$ after stimulus onset (grey shaded area). 


\section{Muscarinic effects on firing rate}

As a previous investigation of cholinergic effects on attentional modulation in visual area V1 reported the muscarinic antagonist scopolamine to have the strongest influence (Herrero et al., 2008), we first focused on its effects in the medial temporal area MT. In total, 128 single units from two monkeys were recorded, of which 100 cells fulfilled the initial criteria regarding data quality (see material and methods for the inclusion criteria).

Our first aim was to quantify the influence of the substance on the measured firing rates. Consequently, we calculated the injection modulation index (IMI) in order to contrast the firing rates during the control block with the firing rates during injection block. The injection modulation index was calculated for all attentional conditions (att-in, att-out, att-fix) separately. In order to test for statistical significance of the injection modulation index (IMI) across conditions, the Wilcoxon signed-rank test was used.

Figure 4 depicts the distribution of the injection modulation index across the recorded cells and highlights the median injection modulation. During the fixation condition, scopolamine reduced the median injection modulation, but this did not reach significance (Wilcoxon signed rank test, signedrank=2491, $\mathrm{zval}=0.2321, \mathrm{~N}=100, \mathrm{p}=0.82$ ). Additionally, during attend-in and attend-out conditions, scopolamine also did not influence firing rates on average, as the median injection modulation did not significantly diverge from o (attend-in: signedrank=2041, zval=-0.72, N=100, $\mathrm{p}=0.47$; attend-out: signedrank=1864, $\mathrm{zval}=-1.23, \mathrm{~N}=100, \mathrm{p}=0.22$ ). 


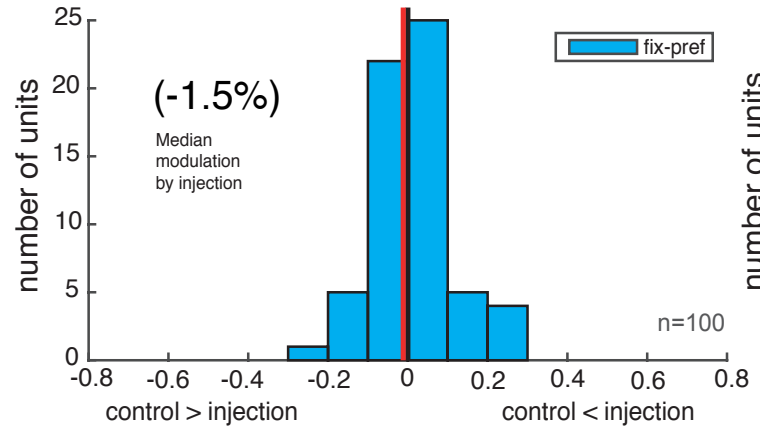

injection modulation

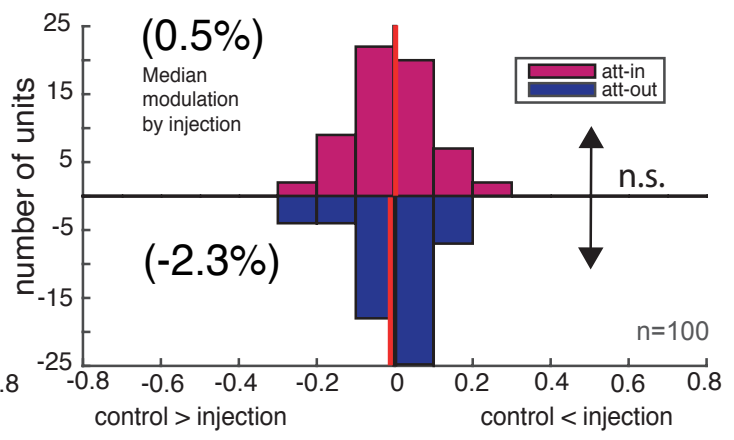

injection modulation

Figure 4. Distribution of injection modulation indices (IMI) for all attention conditions. IMI is defined as the difference in firing rate for two injection blocks (control and injection), divided by the sum. Shown data contains neuronal responses of single units from two monkeys for the preferred stimulus only. The left histogram depicts the IMI distribution for all fixation trials, whereas the right histograms shows the injection effect for attend in (red) and attend out (blue) trials. Red vertical bars indicate median injection modulation.

As several units of both monkeys show an injection modulation index close to zero during the fixation condition (see figure 4) it is unclear whether the injected substance reached the recorded neuron. In order to be able to investigate the influence of the substance on attentional modulation of the recorded cell more closely, we selected in a next step only those neurons that showed a significant influence of injection on the firing rate during the fixation condition (see figure 5, left histogram). Interestingly, only 17 out of 100 recorded neurons showed a significant influence by scopolamine. 

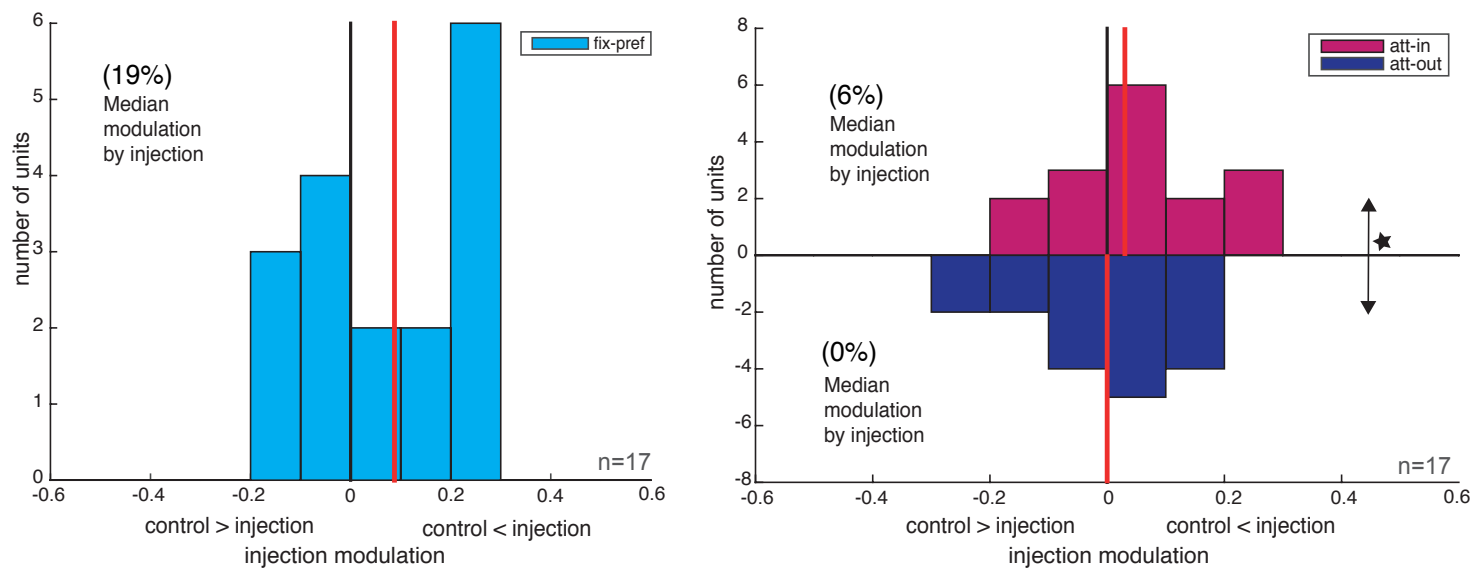

Figure 5. Distribution of injection modulation indices (IMI) during the fixation condition (left) and for attend-in and attend-out task condition (right) for all effected cells by scopolamine injection for two monkeys.

As scopolamine is a non-specific cholinergic antagonist it binds to all types of muscarinic receptor subtypes. Muscarinic receptor types are known to elicit heterogeneous effects (inhibitory or excitatory) based on their variation in location and molecular composition (Wess, 2003). We also observed this heterogeneity in our subpopulation as some cells $(\mathrm{N}=10)$ showed an increase in firing rate with scopolamine injection, and some showed a decrease $(\mathrm{N}=7)$.

On average, the firing rate was increased by $19.2 \%$ (red vertical bar in left histogram of figure 5), but showed no significant difference to zero (Wilcoxon signed rank test: signedrank=110, zval=1.59, N=17, $\mathrm{p}=0.11$ ).

During attend-in and attend-out conditions, the median modulation by injection of this subpopulation did not diverge from o (Wilcoxon signed rank test; attend-in: signedrank=97, zval=1.50, N=17, $\quad \mathrm{p}=0.13$; attend-out: signedrank=65, $\mathrm{zval}=-0.16, \mathrm{~N}=17, \mathrm{p}=0.88$ ). Nevertheless, as shown in the right histogram of figure 5, median injection modulation during attend-in condition showed a significant difference to injection modulation in attend-out condition in a paired test (two-sided Wilcoxon signed rank test; signedrank=111, $\mathrm{zval}=2.22, \mathrm{~N}=17, \mathrm{p}=0.026$ ). 
As we observed diverging effects of scopolamine on firing rates, we further divided the subgroup based on whether scopolamine increased or decreased the firing rate during fixation trials. For these subpopulations we again calculated the influence by injection for attend-in and attend-out conditions (see Figure 6).

a)

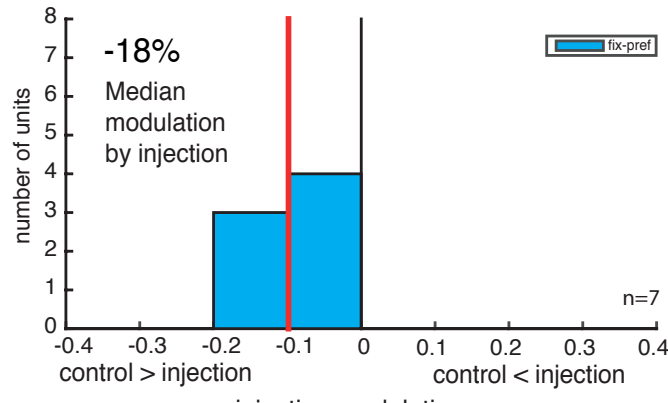

injection modulation

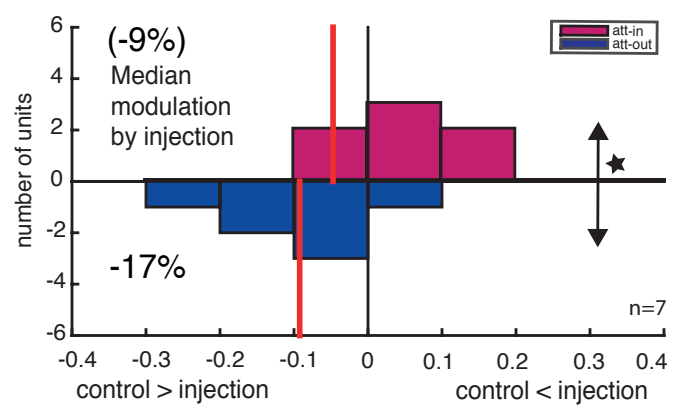

injection modulation

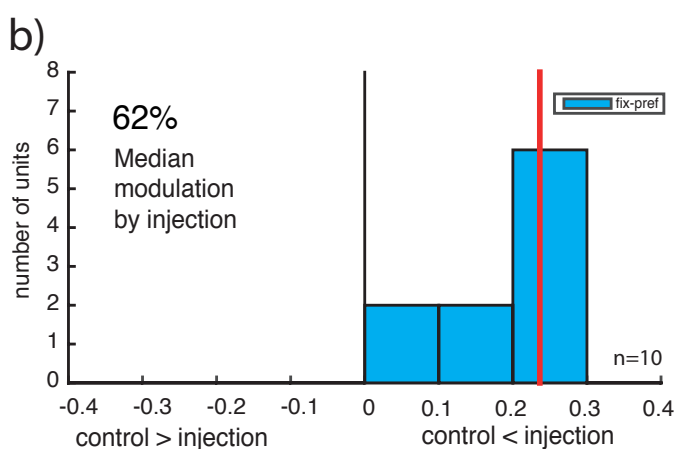

injection modulation

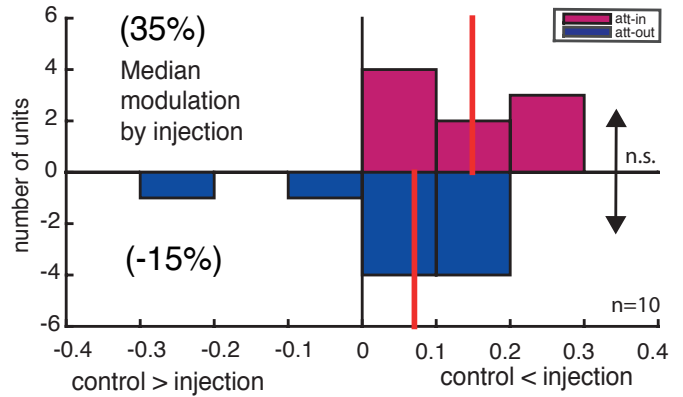

injection modulation

Figure 6. Injection modulation distributions in attend-in and attend-out condition for two subgroups of scopolamine effected cells. a) Cells that showed reduced firing rate with scopolamine injection during fixation condition; b) cells that showed enhanced firing rate with scopolamine.

The subpopulation of cells which each showed a significant decrease in firing rate at the single-cell level during the fixation condition also showed a decrease of $9 \%$ in firing rate due to injection during attend-in condition (Wilcoxon signed rank test; signedrank $=4, \mathrm{~N}=7, \mathrm{p}=0.11$ ). In attend-out condition the negative influence by scopolamine is again more prevailing, showing approximately the same decrement of $17 \%$ in firing rate as in the fixation condition compared to 0 (Wilcoxon signed rank test; signedrank=1 N=7, $\mathrm{p}=0.03$ ). 
The other subpopulation, showing an increase in firing rate with scopolamine injection in fixation condition, was much stronger but not significantly influenced by scopolamine during the attend-in condition. Here cells show a median increase of $35 \%$ on firing rate. (Wilcoxon signed rank test; signedrank $=45, N=10, p=0.11)$. When the monkeys deploy spatial attention outside of the receptive field, average influence by scopolamine dropped to $15 \%$ (Wilcoxon signed rank test attend-out vs. 0 ; signedrank=35, N=7, $\mathrm{p}=0.16$; attend-out vs. attend-in: signedrank=10, N=7, p=0.16).

\section{Muscarinic effects on attentional modulation}

In order to test for cholinergic effects on attentional modulation, an attentional modulation index (AMI) was calculated and compared for control and injection blocks. Figure 7 compares the attentional modulation distribution of the recorded cells fulfilling the first criteria (sufficiently high firing rate, sufficiently many trials, direction selectivity).
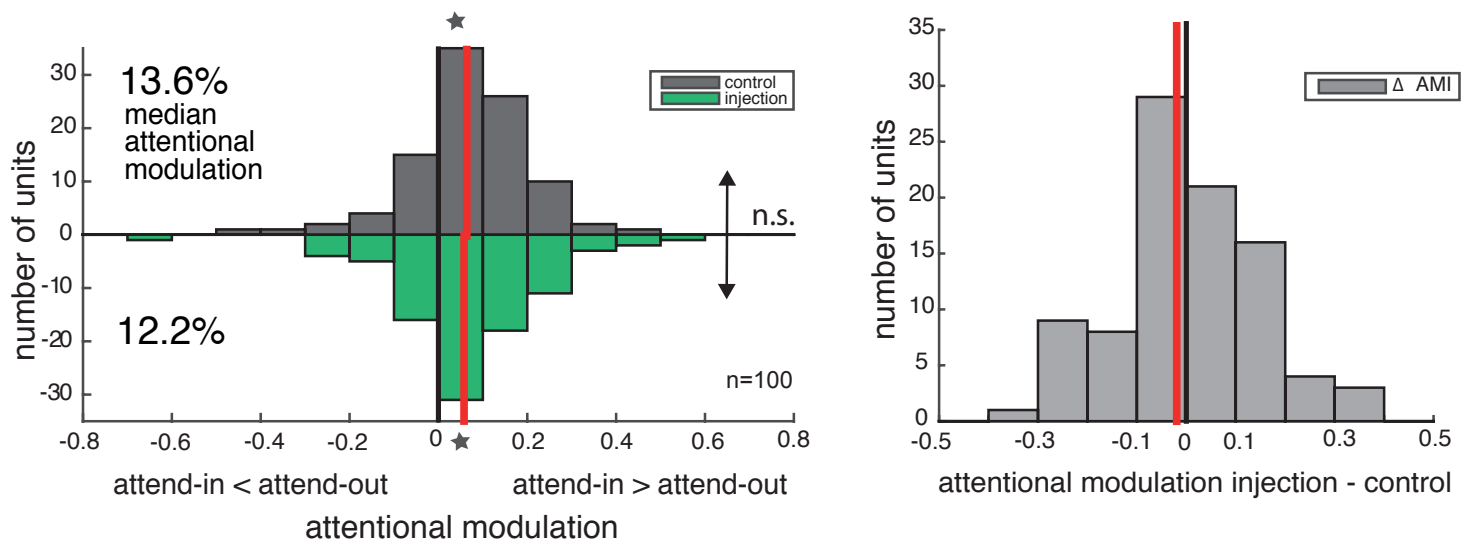

Figure 7. Distribution of attentional modulation index (AMI) for control and scopolamine injection block (left). The paired difference of attentional modulation indices for the two blocks, control and injection, is shown in the right histogram. Red vertical bars indicate median attentional modulation. 
During the control block the cells were significantly influenced by spatial attention, showing a median modulation of $13.6 \%$ (Wilcoxon signed rank test; signedrank $=3981$, $\left.\mathrm{zval}=5.26, \mathrm{~N}=100, \mathrm{p}<10^{-7}\right)$. During the block of scopolamine injection the median attentional modulation decreased to $12.2 \%$ attentional modulation but remained significantly different to zero (signedrank=3031, $\mathrm{zval}=4.21, \mathrm{~N}=100, \mathrm{p}<10^{-5}$ ). In order to compare the attentional modulation during the control block with the injection block, we calculated the difference of attentional modulation (delta AMI), subtracting cell-wise the attentional modulation during injection block with attentional modulation during control block. The median delta AMI is slightly reduced by scopolamine injection, however it did not reach significance (paired, two-sided Wilcoxon signed rank test; signedrank=2186, $\mathrm{zval}=0.18, \mathrm{~N}=100, \mathrm{p}=0.8$ ).

In a next step, we again selected only those cells showing a significant influence by the injection of the muscarinic antagonist scopolamine at the single-cell level in the fixation condition. With this subpopulation, the modulatory effect of attention was significant, with a median increase of $12.2 \%$ observed in firingrates due to attention (Wilcoxon signed rank test; signedrank=124, $\mathrm{zval}=2.25$, $\mathrm{N}=17, \mathrm{p}=0.02$ ). When scopolamine was injected, a significant increase in attentional modulation to a value of $17 \%$ was observed (paired, two-sided Wilcoxon signed rank test; signedrank=25, $\mathrm{zval}=-2.2, \mathrm{~N}=100, \mathrm{p}=0.03$ ). 

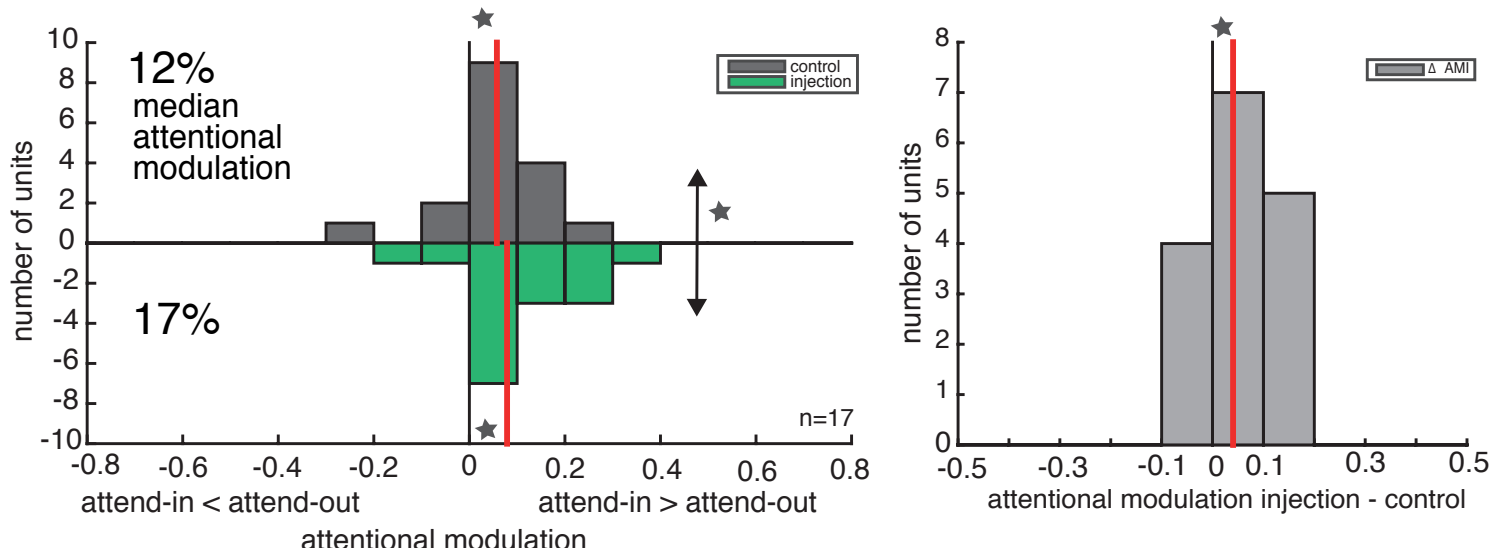

Figure 8. Histogram of attentional modulation index for control and scopolamine injection block for cells showing significant influence by injection at the single-cell level. The difference of these two experimental blocks is illustrated in the right histogram. Red vertical bars indicate the median change of the population.

If we again divide the subgroup based on increasing or decreasing influences of scopolamine (see Figure 9), it become apparent that the cell group showing a stronger influence by scopolamine injection (histogram b in Figure 9) are stronger modulated by attention.
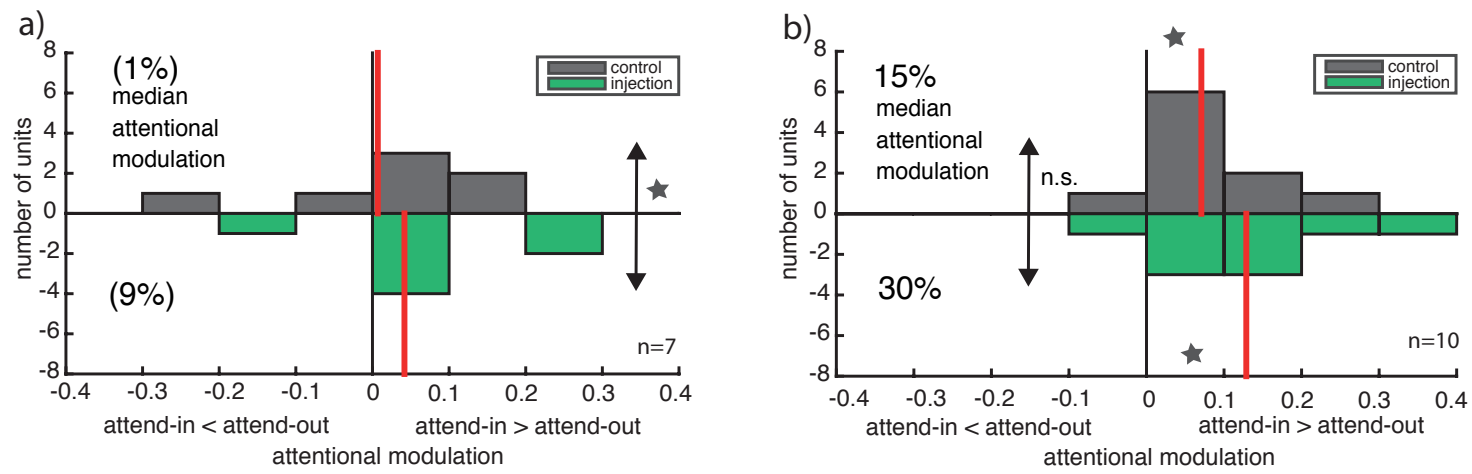

Figure 9. Histograms of the subpopulation significantly modulated cells by scopolamine, grouped by whether they showed a scopolamine-mediated decrease (left) or increase (right) in firing rate during the fixation condition. Red vertical bars show median change of the subpopulation. 
However, also the subgroup showing decreased firing rate due to scopolamine injection in the control block, showed on average a positive shift of the attention modulation index during the injection block (paired, two-sided Wilcoxon signed rank test; signedrank=2, $\mathrm{N}=7, \mathrm{p}=0.047$ ).

To summarize the effects of local scopolamine injection, we can report that on average, local application of scopolamine did not lead to any change in attentional enhancement. However, the subpopulation of cells affected by scopolamine in the fixation condition showed a significant increase in attentional modulation when scopolamine was injected.

\section{Muscarinic effects on reaction time}

We were interested whether the local application of scopolamine induced a behavioral change. We calculated and compared reaction times during control and scopolamine injection blocks for all attentional conditions (attend-fix, attend-out, attend-in). For both monkeys separately, a repeated-measures ANOVA with main factors injection and attention was performed to investigate the overall influence of scopolamine injection and experimental condition on reaction time. For both monkeys the injection of scopolamine had no significant effect on reaction time (monkey $\mathrm{p}: \mathrm{F}(1,50)=0.106, \mathrm{p}=0.746, \eta^{2}=0.00212$; monkey o: $\left.\mathrm{F}(1,48)=1.48, \mathrm{p}=0.23, \eta^{2}=0.0299\right)$. Figure 10 depicts the distribution of the reaction time change when scopolamine is injected. 


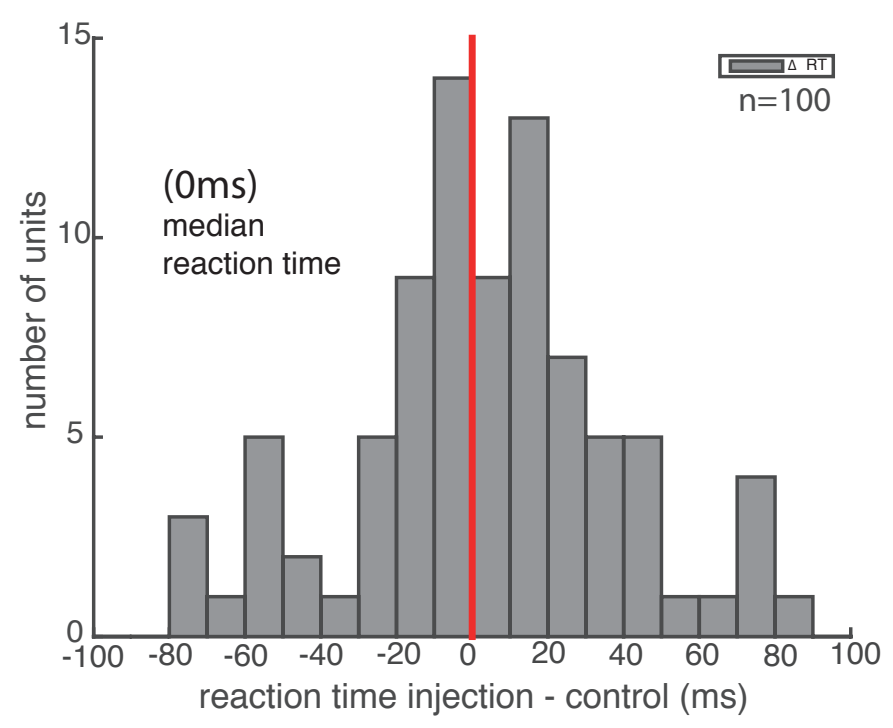

Figure 10. Reaction time difference distribution when scopolamine is injected for the attend-in condition. The vertical bar depicts the median change of reaction time.

The experimental conditions had a main effect on reaction times for both monkeys (monkey p: $\mathrm{F}(2,100)=23.6, \mathrm{p}=2.66 \mathrm{e}-05, \eta^{2}=0.32$; monkey o: $\mathrm{F}(2$, 96) $\left.=257, \mathrm{p}=0, \eta^{2}=0.843\right)$. No interaction was found for the two main factors (monkey p: $\mathrm{F}(2,100)=0.0855, \mathrm{p}=0.815, \eta^{2}=0.00171$; monkey o: $\mathrm{F}(2,96)=0.966$, $\left.\mathrm{p}=0.331, \eta^{2}=0.0197\right)$.

Additionally, we were interested whether there is a relationship between scopolamine-induced change in firing rate and behavioral performance. Therefore a linear regression model was fitted $(y=\alpha+\beta \chi)$. Here we tested for significant linear regression relationship between the response variable and the predictor variables by performing the test statistic for the F-test on the regression model. The p-value of the F-test on the model indicates significance.

Figure 11 shows this correlation for the full population (a) and for the subpopulation (b) of scopolamine injection. 

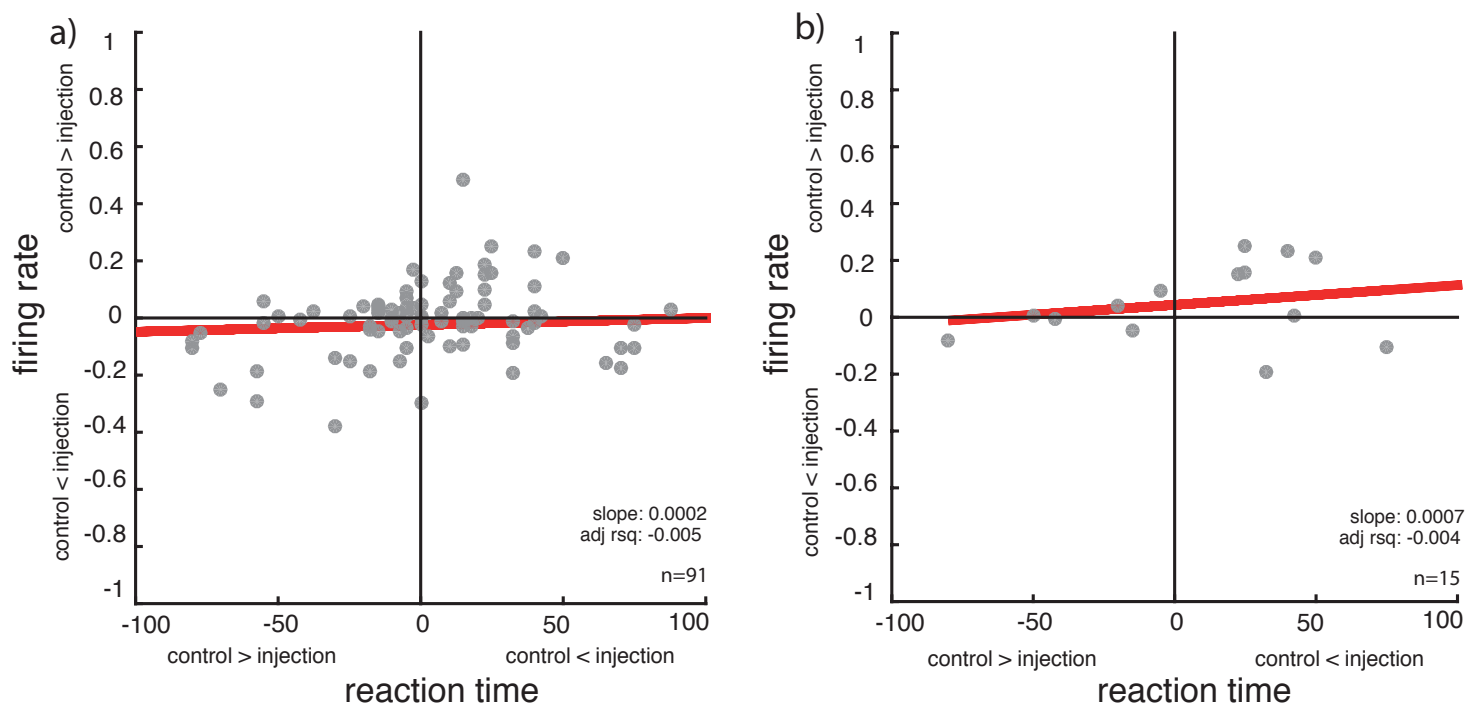

Figure 11. Correlation of neuronal firing rate and reaction time for a) full population and b) subpopulation of the sig. affected cells. Dots represent the averaged reaction time difference plotted against the average injection modulation index for every recorded cell. Slope: estimate of the slope for the linear regression; adj rsq: adjusted R-squared.

We did not find a significant linear regression relationship between the response variable and the predictor variables (F-statistic vs. constant model: 0.465, $\mathrm{p}$-value $=0.497$ ). We also failed to find a significant linear relationship in the subpopulation showing significant influence by injection (F-statistic vs. constant model: 0.942, $\mathrm{p}$-value $=0.35$ ).

\section{The effect of acetylcholine on single unit activity}

In order to investigate a general cholinergic involvement in attentional modulation, we injected acetylcholine in the direct vicinity of the recorded neuron. We performed the same analysis for this substance as for the muscarinic antagonist scopolamine. Therefore we calculated in a first step the PSTH for both monkeys separately, during the control block, where no substance was injected. As illustrated in Figure 12, both monkeys show clear attentional enhancement of their firing rate, as the firing rates for attend-in 
condition are clearly elevated compared to firing rates in attend-out or attendfix conditions (red vs. blue lines in Figure 12). As both monkeys show a similar effect of spatial attention on their firing rate, we pooled the data from the two monkeys. Subsequent analysis is based on firing-rates in the time window of 300-800 ms after stimulus onset (grey shaded area).
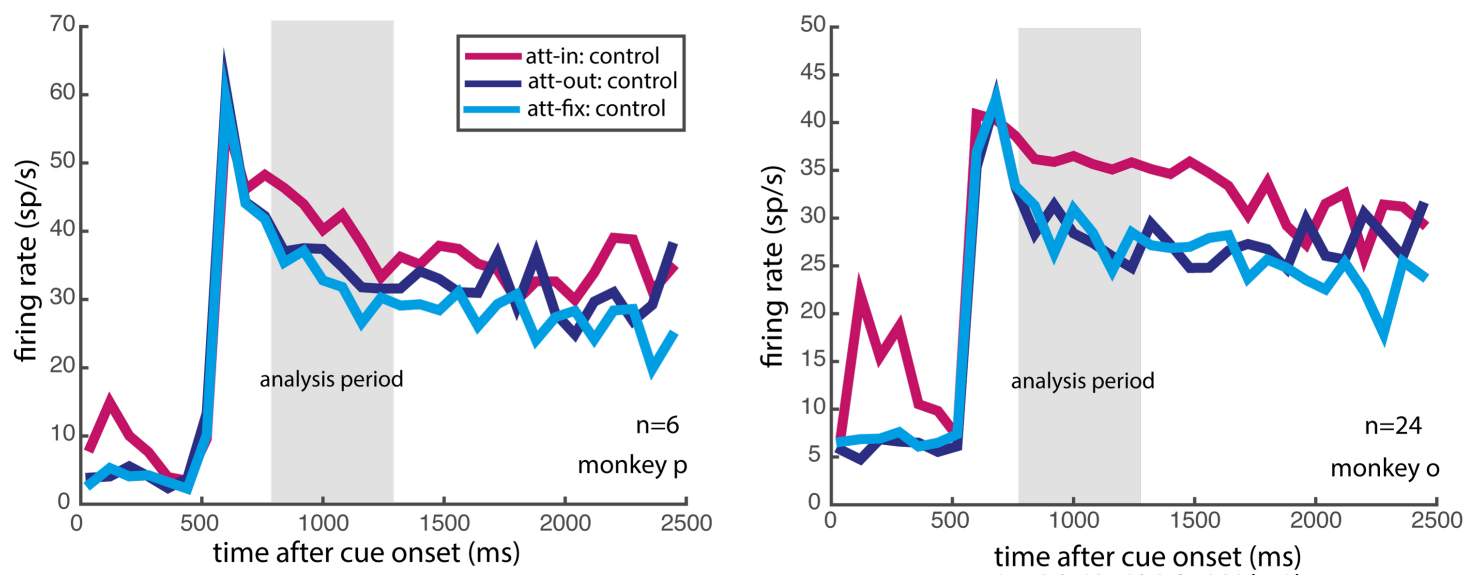

Figure 12. Effect of spatial attention on neuronal firing rate across trial time course. Peristimulus time histograms were calculated for the control condition for the acetylcholine cell population of each monkey separately (left: monkey p; right: monkey o). The analysis period is defined as 300-80oms after stimulus onset (grey shaded area).

We calculated in a second step the injection modulation index (IMI) to investigate the influence of the substance on the measured firing rates. The IMI is calculated for every attentional condition separately.

Figure 13 shows the effect of acetylcholine injection on the firing rate at population level in the fixation condition for the preferred direction. During the fixation condition, in which the monkeys performed a task at fixation while ignoring stimuli with the cell's preferred direction, the median firing rate was not significantly affected by acetylcholine injection (Wilcoxon signed rank test; $\mathrm{zval}=0.52$, signedrank $=226, \mathrm{~N}=30, \mathrm{p}=0.6$ ). When the monkey attended inside the neurons' receptive field, the neuronal response increased on average when acetylcholine was injected, reaching a median modulation of $10.7 \%$. However, 
this effect is not significantly different to zero (Wilcoxon signed rank test; $\mathrm{zval}=1.2$, signedrank=291, $\mathrm{N}=30, \mathrm{p}=0.2$ ). When monkeys attended outside the receptive field, the neurons were not affected by injection, showing a median injection modulation of $0.4 \%$ (Wilcoxon signed rank test; zval=0.6, signedrank=215, $\mathrm{N}=30, \mathrm{p}=0.5$ ).
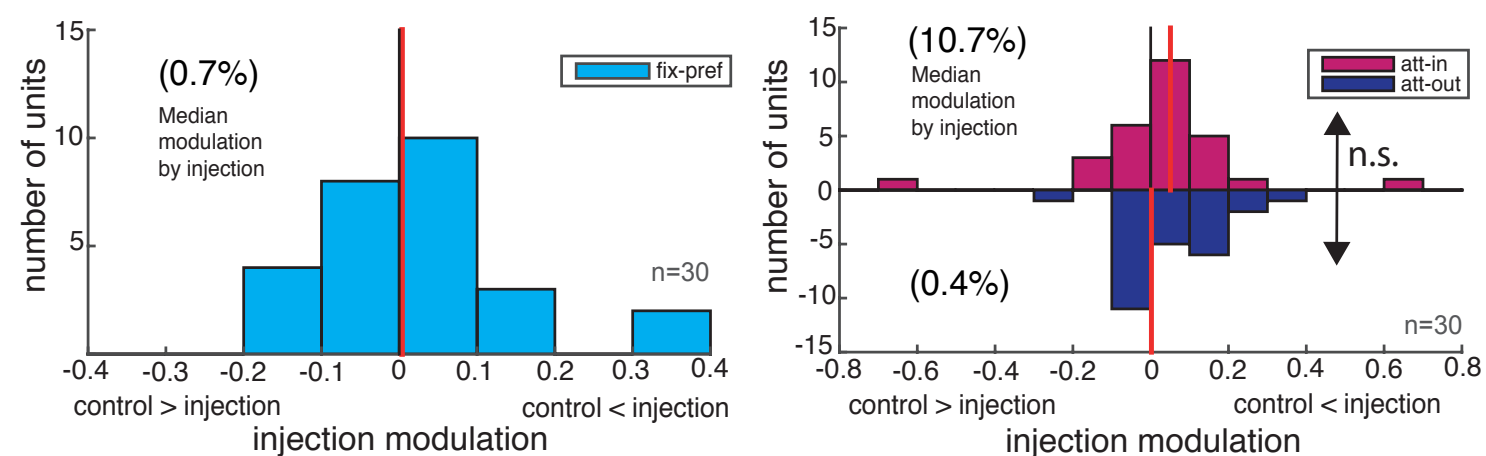

Figure 13. Distributions of injection modulation indices (IMI) during the fixation condition (left) and attend-in/ attend-out (right) for all effected cells by acetylcholine injection for two monkeys. Red vertical bars indicate the median modulation by injection.

As we did not observe significant effects of acetylcholine injection on average, we selected only cells that showed a significant effect by injection, in order to confirm that the substance reached the recorded neuron. Only 6 out of 30 cells showed a significant injection effect during fixation condition (see figure 14). Also with this subset of neurons, acetylcholine had on average no influence on firing rate during fixation condition (Wilcoxon signed rank test; signedrank=11, $\mathrm{N}=6, \mathrm{p}=1$ ). Similar to the effect seen in fixation condition, the subpopulation of neurons did not show a significant median effect of acetylcholine injection during attend-in and attend-out conditions (Wilcoxon signed rank test; attendin vs. o: signedrank $=9, \mathrm{~N}=6, \mathrm{p}=0.8$; attend-out vs. o: signedrank=9, $\mathrm{N}=6$, $\mathrm{p}=0.8$; attend-out vs. attend-in: signedrank $=9, \mathrm{~N}=6, \mathrm{p}=0.8$ ). 


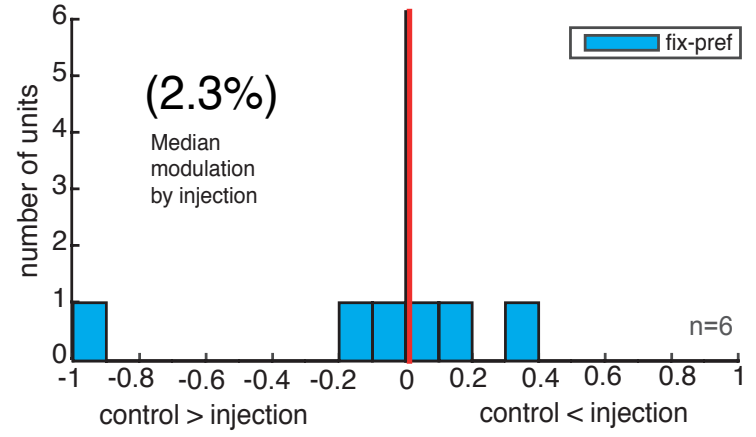

injection modulation

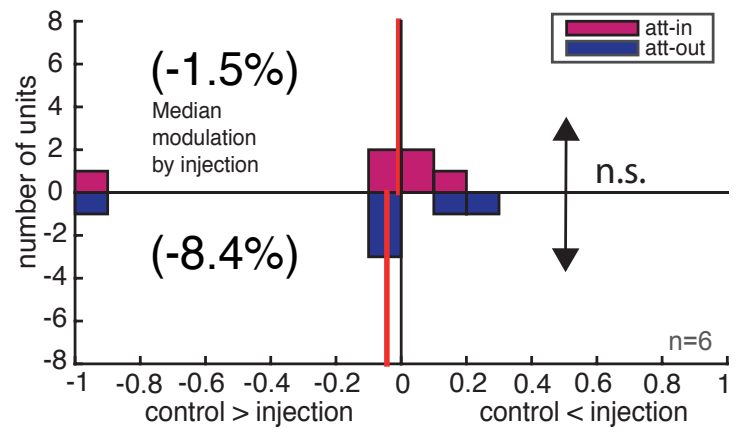

injection modulation

Figure 14. Injection modulation distributions in attend-fix (left), attend-in (right top) and attend-out (right bottom) condition for the subgroup of cells affected by acetylcholine injection at the single-cell level. Red vertical bars indicate the median modulation by injection.

\section{Cholinergic effects on attentional modulation}

In a next step we investigated the influence of local acetylcholine injection on attentional modulation. Therefore, we calculated the attention modulation index (AMI) and compared control with injection blocks. During the control block the cells were significantly influenced by spatial attention, showing a median modulation of $19.6 \%$ (Wilcoxon signed rank test; signedrank=395, $\left.\mathrm{zval}=3.34, \mathrm{~N}=30, \mathrm{p}<10^{-4}\right)$.
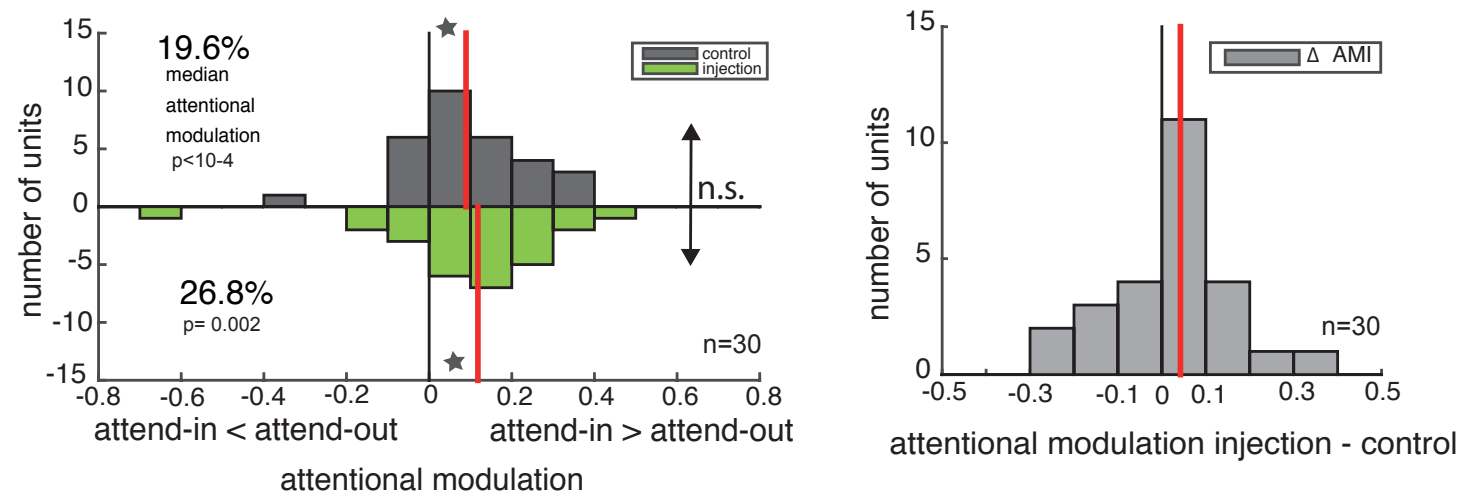

Figure 15. Distribution of attentional modulation index (AMI) for control and acetylcholine injection block (left). The paired difference of attentional modulation indices for the two blocks, control and injection, is shown in the right histogram. Red vertical bars indicate median attentional modulation. 
When the general agonist acetylcholine was injected, the attentional modulation increased to a median modulation of $26.8 \%$, still being significantly different to zero (Wilcoxon signed rank test; signedrank $=317$, zval=3.08, $N=30, p=0.002$ ). Although the acetylcholine induced increase in attentional modulation is relatively strong, it did not reach significance (Wilcoxon signed rank test; signedrank=159, zval=-0.72, N=30, $\mathrm{p}=0.47)$.

Again, we selected only those cells, which showed a significant influence by acetylcholine injection during fixation. As shown in Figure 16, the median attention modulation index stays constant at a level of approximately $22 \%$ for control and injection block.
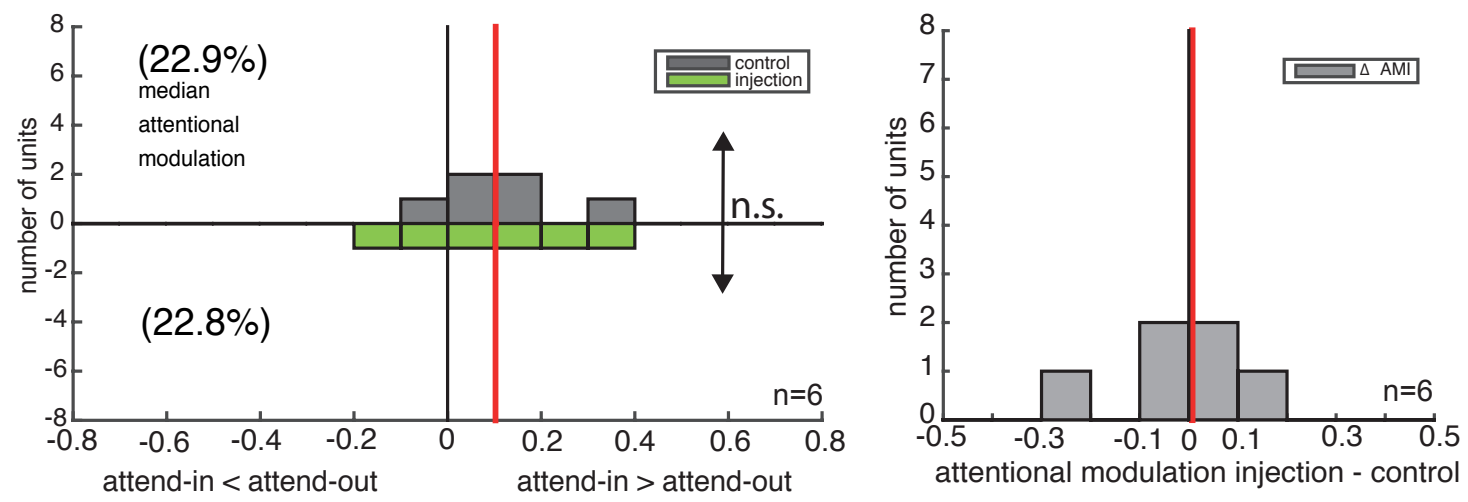

attentional modulation

Figure 16. Distribution of attentional modulation index (AMI) for control and acetylcholine injection block (left) of the significantly effected cells. The paired difference of attentional modulation indices for the two blocks, control and injection, is shown in the right histogram. Red vertical bars indicate median attentional modulation.

However, due to the low number of cells it is not significantly different to zero (Wilcoxon signed rank test; control vs. $\mathrm{o}$ : signedrank=20, $\mathrm{N}=6, \mathrm{p}=0.6$; injection vs. 0 : signedrank $=17, \mathrm{~N}=6, \mathrm{p}=0.2$; control vs. injection: signedrank $=11, \mathrm{~N}=6$, $\mathrm{p}=1$ ). Because the total amount of significantly affected cells is very small, we 
did not further subdivide into positively and negatively affected cells, as we did for the scopolamine injection.

\section{Behavioral effects of acetylcholine injection}

Repeated-measures ANOVA with main factors injection and attention were performed to investigate the overall influence of acetylcholine injection and experimental condition on reaction times.

Acetylcholine injection had no significant main effect on reaction times ( $\mathrm{F}(1$, 29) $=0.133, p=0.718, \eta^{2}=0.00457$ ), whereas spatial attention revealed significant influence $\left(\mathrm{F}(2,58)=26, \mathrm{p}=0.000126, \eta^{2}=0.472\right)$. No interaction was found between the two main factors $\left(\mathrm{F}(2,58)=2.51, \mathrm{p}=0.134, \eta^{2} 0.0797\right)$.

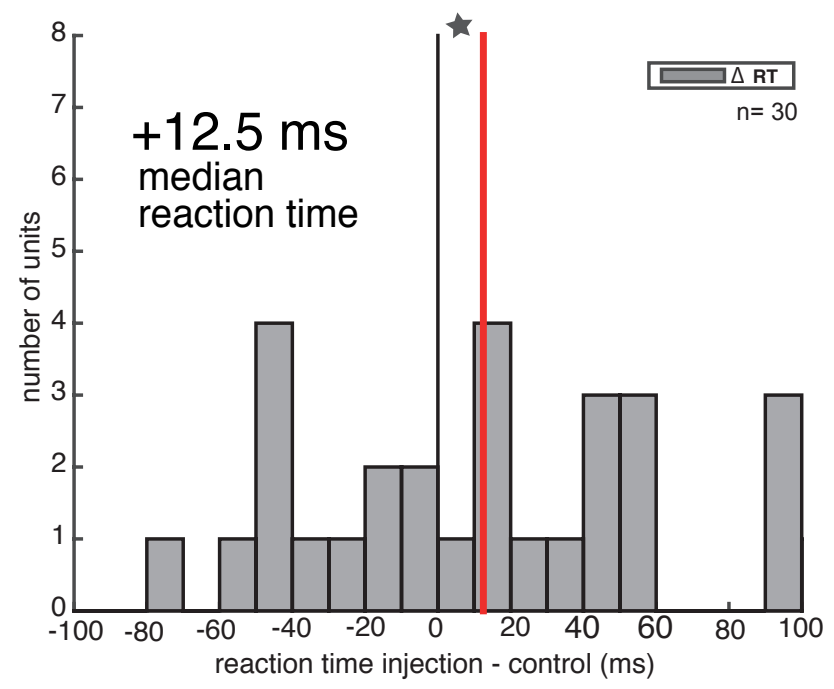

Figure 17. The distribution of reaction times (injection-control) across all recording sessions for attend-in task condition. The vertical bar illustrates the median reaction time for all recording sessions.

In order to investigate the behavioral effects of local acetylcholine injection we calculated and compared the difference of the median reaction times for attendin trials between injection and control blocks for every recording session 
separately (see Figure 17). Although the distribution is relatively broad, the median change in reaction time is significantly affected by acetylcholine injection (Wilcoxon signed rank test; signedrank $=465, \mathrm{zval}=4.8, \mathrm{~N}=30, \mathrm{p}<10^{-}$ 5), showing an increase in reaction time with injection.

We further explored the change in behavioral performance with respect to the change in firing rate. Therefore we calculated the correlation between acetylcholine-induced change in firing rate and change in reaction time, by fitting a linear regression model $(y=\alpha+\beta \chi)$. Figure 18 shows this correlation for the full population (a) and for the subpopulation of significantly affected cells (b) of acetylcholine injection for both monkeys together.

We did not find significant linear regression relationship between the response variable and the predictor variables (F-statistic vs. constant model: 1.02, pvalue $=0.322$ ). Also with the subpopulation showing significant influence by injection, we did not find significant linear relationship (F-statistic vs. constant model: 0.931 , $\mathrm{p}$-value=0.38). 


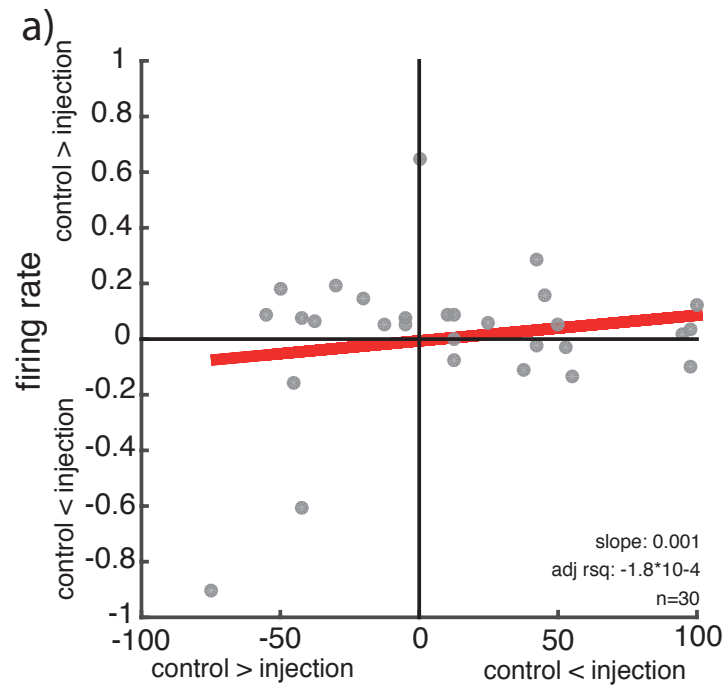

reaction time

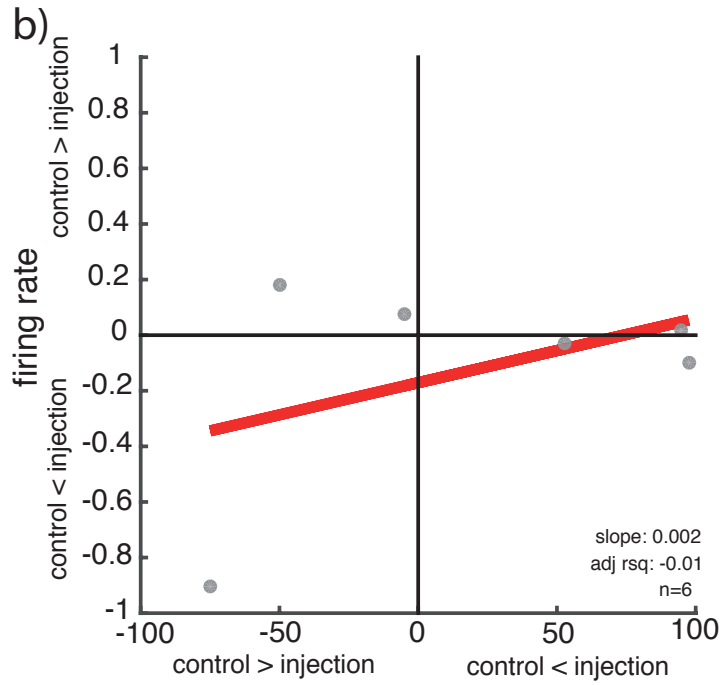

reaction time

Figure 18. Correlation of neuronal firing rate and reaction time for a) full population and b) subpopulation of the sig. affected cells. Dots represent the averaged reaction time difference plotted against the average injection modulation index for every recorded cell. Red line depicts the linear regression. Values of the slope and the adjusted Rsquare are shown in the right corner.

\section{Nicotinic involvement in attentional modulation}

As the muscarinic receptor type showed only weak or no influence on attentional modulation, we were interested whether the nicotinic receptor type is involved in attentional modulation. Therefore we injected mecamylamine, a nonselective nicotinic antagonist, in the direct vicinity of the recording electrode. Data collection is ongoing and so far we have only manipulated a few cells pharmacologically with this substance. Figure 19 depicts three example cells and shows the variety of mecamylamine action on firing rate for the different attentional conditions over the course of the experiment. The grey shaded area shows the block of mecamylamine injection, beginning with the first injection and ending one minute after the last injection. Averaged firing rates for the different attentional conditions during the three experimental blocks (control, injection, recovery) are shown as horizontal bars of different color. In example cells a and $\mathrm{b}$ an injection of mecamylamine led to an increase 
in median firing rate for most attentional conditions. But we additionally observed a contradictory effect, in which firing rates dropped dramatically during the injection period and no recovery was observed (see Figure $19 \mathrm{c}$ ). It is noticeable that all cells showed an increase in firing rate for the attend-in condition (pink horizontal bar). This indicates already that the nicotinic receptor might be involved in processes of attentional modulation, but this needs to be confirmed on a population level.
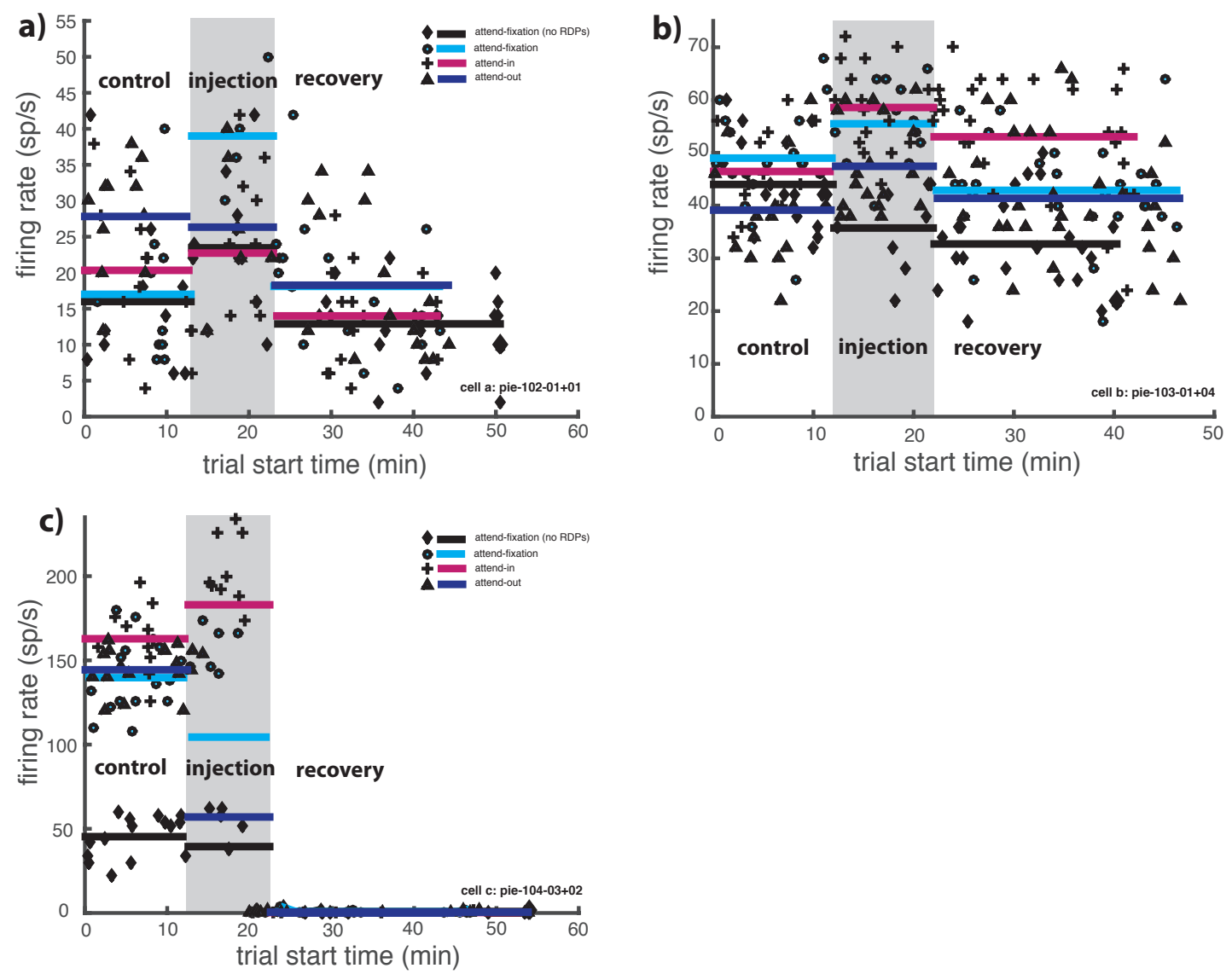

Figure 19. Three example cells are shown with mecamylamine injection. The trial-averaged firing rate of three sample cells over the course of the experiment is shown for the preferred stimulus for all three attentional conditions plus the condition where only a fixation point is shown. The $\mathrm{x}$-axis depicts the trial start time in minutes and the y-axis shows the unit's firing 
rate in spikes per seconds. Symbols ( + attend-in, $\bullet$ attend-fix, $\Delta$ attend-out, $\bullet$ att-fix (noRDP)) represent the neuron's firing rate within the analysis period in every successfully performed trial, and horizontal lines (pink: attend-in, light blue: attend-fix, dark blue: attend-out, black: att-fix (noRDP)) show average firing rate for the three different experimental blocks (control, injection, recovery). The grey shaded area shows the injection block, beginning with the first injection and ending one minute after the last injection. During the injection block $6 \mathrm{nl}$ of 0.1 molar mecamylamine were injected every minute with an injection velocity of $2 \mathrm{nl} / \mathrm{s}$.

\section{Control experiments - saline injection}

In order to exclude an influence of the injection process per se on the neuronal firing rate, we injected saline as a control substance, following the same protocol as the other injections. On average the firing rates of the 28 recorded neurons of two monkeys were not significantly altered by injection during fixation condition (Wilcoxon signed rank test; $\mathrm{zval}=0.34$, signedrank=218, $\mathrm{N}=\mathbf{2 8}$, $\mathrm{p}=0.7$ ). Additionally, we could exclude the influence of the injection process on attentional enhancement, as the attention modulation index was not significantly changed with saline injection (paired two-sided Wilcoxon signed rank test; zval=0.24, signedrank=199, $\mathrm{N}=28, \mathrm{p}=0.8$ ). 


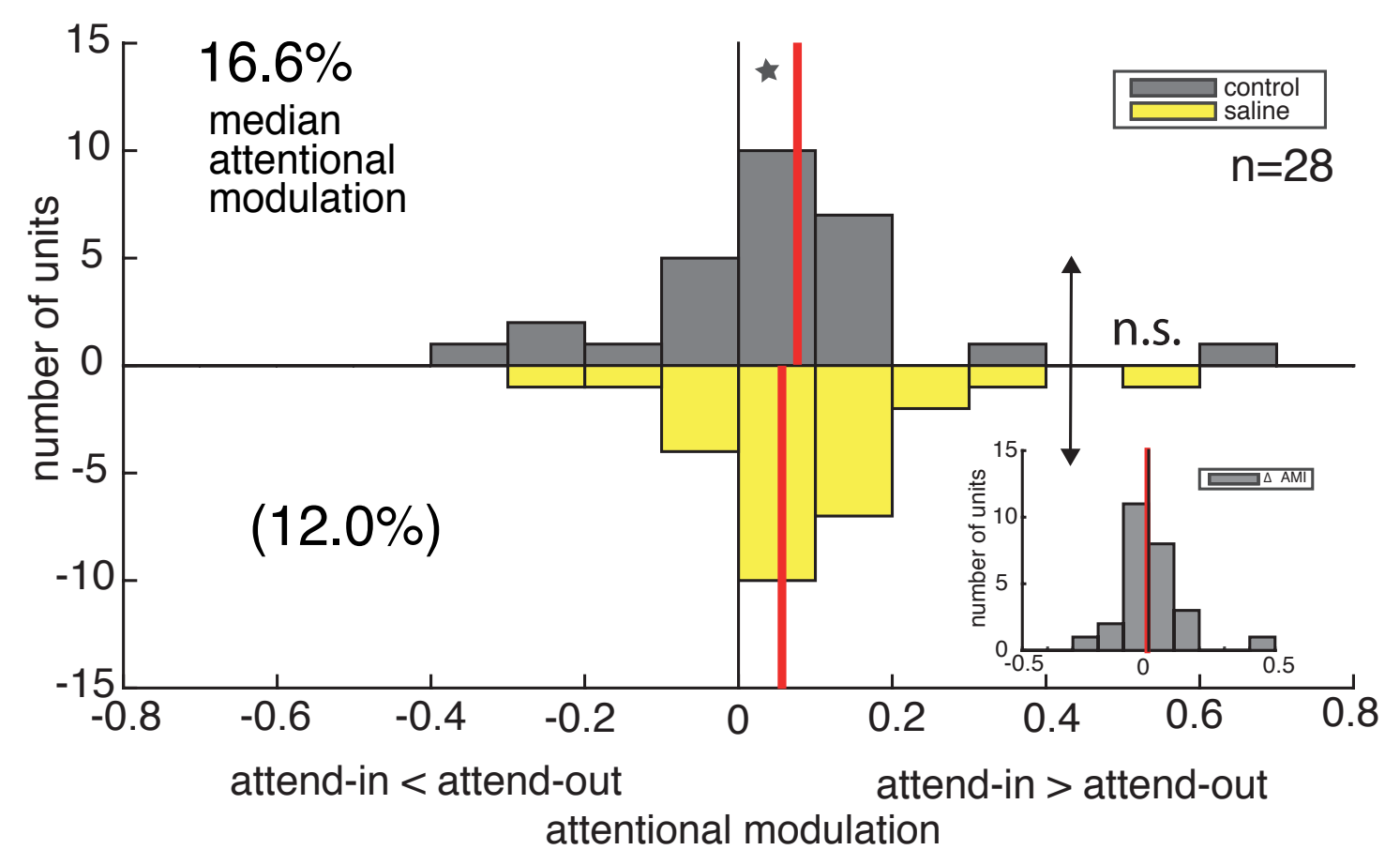

Figure 20. Attentional modulation for the control and the saline injection block. The histogram shows the distribution of spatial attention. The comparison of the two blocks is illustrated by the difference of the corresponding distributions.

As a last step we investigated the influence of the injection process on monkey behavior. RmANOVA revealed a significant main effect of attention on the reaction time, whereas the injection had no significant effect on behavior. No interaction was found (injection: $\mathrm{F}(1,23)=0.591, \mathrm{p}=0.45, \eta^{2}=0.025$; attention: $\mathrm{F}(2,46)=19.9, \mathrm{p}=0.000774, \eta^{2}=0.464$, injection $\mathrm{x}$ attention: $\mathrm{F}(2,46)=2.29$, $\left.\mathrm{p}=0.151, \eta^{2}=0.0904\right)$. 


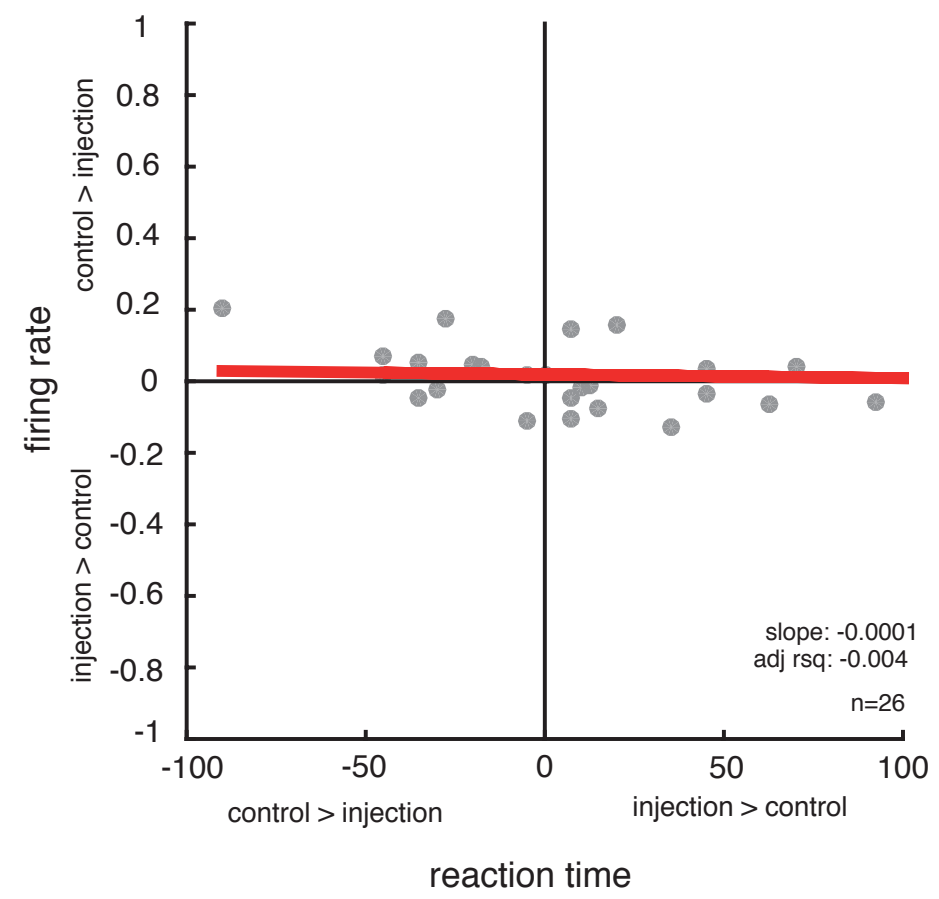

Figure 21. Correlation of saline induced firing rate change and reaction time change. Red line indicates the linear regression. Slope: estimate of the slope for the linear regression; adj rsq: adjusted R-squared.

As shown in figure 21, the fitted linear regression model revealed only a weak relationship between behavior and injection induced firing rate (slope=-0.0001, $\mathrm{r}^{2}=0.004$ ) and did not reach significance (F-statistic vs. constant model:0.0937, p-value $=0.762$ ).

\section{Discussion}

The aim of this study was to investigate the local cholinergic influence on attentional modulation in the medial temporal area (MT) of the macaque monkey. Therefore, the general agonist acetylcholine, a muscarinic antagonist, or a nicotinic antagonist was injected in the direct vicinity of the recording 
electrode, while the monkey performed a task that required top-down spatial attention.

With our task design we could confirm an increase in neuronal response in area MT when spatial attention was deployed within the neurons' receptive field. The obtained attention-induced increase in neuronal response ranged from 12-22\% and therefore replicates the average achieved enhancement of approx. 20\% in area MT (Treue and Maunsell, 1999).

Although this attention-induced effect on neuronal response is a well-known and well-studied effect, the underlying neuromodulatory involvement triggering these effects is largely unspecified. Acetylcholine was shown to elicit a multiplicative increase in neuronal gain in area V1 (Disney et al., 2007) and area MT in anesthetized macaques (Thiele et al., 2012) and is therefore, among other reasons, proposed as a main regulative neurotransmitter of selective attention (Klinkenberg et al., 2011). But the exact involvement is still unclear as the above-mentioned studies were performed in anesthetized monkeys.

\section{Muscarinic receptor influence on firing rate}

In a first step, we investigated the effect on firing rate for the whole cell population during the injection of the muscarinic antagonist scopolamine and compared it to the averaged firing rate measured before the injection was performed (control block). On average, we did not observe a change in firing rate due to injection. As scopolamine is a non-specific muscarinic antagonist, it binds to all types of the muscarinic receptor subtype. Muscarinic receptor types are therefore known to elicit heterogeneous effects (inhibitory or excitatory) based on their variation in location and molecular composition (Wess, 2003). When we selected only cells significantly affected by scopolamine, we also observed this heterogeneity in our subpopulation as some cells showed an increase in firing rate with scopolamine injection, and some showed a decrease. 


\section{Muscarinic receptor contribution to attentional modulation}

Although the cholinergic projection neurons, originating in the nucleus basalis of Meynert (Mesulam et al., 1983), form clusters providing a topographically organized network of cholinergic innervations in the cortex, the spatial distribution of acetylcholine is thought to be widespread. Thus, the specific involvement of acetylcholine in attentional modulation is likely triggered by a selective expression of ACh receptors (AChRs) within the sensory areas targeted by top-down attention. We primarily investigated the muscarinic receptor's involvement in attentional modulation, as it was shown to be strongly involved in attentional modulation in V1 (Herrero et al., 2008).

Although we detected a strong influence of spatial attention on the neuronal firing rate, the attentional modulation was only marginally diminished by injection of the muscarinic antagonist scopolamine for the full data set, and this effect did not reach significance.

In primary visual cortex (V1), it was shown that the muscarinic receptor type is involved in attentional modulation (Herrero et al., 2008), as a blockage of the muscarinic receptor subtype led to a reduction in attentional modulation. This result suggests that the attentional effect on neuronal response is either directly modulated by the muscarinic receptor type, which is predominantly found on inhibitory cells in V1 (Disney et al., 2006), or is more indirectly mediated through subsequent changes in the local cortical microcircuit. As spatial attention effects on neuronal responses are thought to increase in magnitude with every successive step up the visual hierarchy (Maunsell and Cook, 2002), a stronger and more direct influence of the muscarinic receptor type would then be expected in area MT compared to area V1. However, for the total population of recorded cells, it was not observed in area MT.

It could be argued, that this observed lack of muscarinic involvement in attentional modulation is due to an unsuccessful injection process, resulting in 
an absence of scopolamine close to the recorded neuron. In order to elude this constraint, we examined only those cells that showed a significant influence on firing rate by scopolamine injection during the fixation condition. Approximately one half of the significantly affected cells showed an increase in their firing rate when the muscarinic receptor type was blocked, whereas the other half showed reduced firing. This result is in line with anatomical results showing that the muscarinic receptor type m1, which is proposed to be involved in attentional modulation, is located on inhibitory and excitatory neurons in area MT (Disney et al., 2014).

On average, the significantly affected subpopulation showed a significant increase in attentional modulation with scopolamine injection, an effect opposite to that found in area V1 (Herrero et al., 2008). This effect could be partially explained by the competitive binding characteristics of scopolamine. This means that the available acetylcholine, which would have otherwise bound to the pharmacologically blocked muscarinic receptors, is now available in the synaptic cleft and can bind to other receptors. This in turn can lead to an increased activity of nicotinic receptors and can convey a misleading impression regarding the contribution of the receptor targeted by the pharmacological manipulation. However, other scenarios are also plausible, which are described below in the section 'Speculations on local cholinergic effects'.

\section{Cholinergic receptor influence on firing rates}

We report that our pharmacological manipulation, which increased the concentration of the neuromodulator acetylcholine in the local circuitry, showed heterogeneous effects, as some neurons showed an increase and some a decrease in firing rate. Based on results, showing an increase in GABA release with iontophoretic acetylcholine injection in area V1 (Disney et al., 2012), it is expected that a cholinergic boost would lead to an inhibitory tone in V1 (Disney 
et al., 2014). In contrast to this observation, it has been suggested, that the release of acetylcholine induces an increased release of glutamate, the main excitatory neurotransmitter in the brain, in the visual cortex, which in turn leads to an increase in firing rate (Herrero, 2011).

\section{Cholinergic effect on attentional modulation}

With the current data set, we observed an enhancement in attentional modulation with acetylcholine injection. Although the median attentional modulation increased by $35 \%$ with acetylcholine injection, this effect did not reach significance. We expect this effect to become significant with more cells recorded. This result suggests that acetylcholine is involved in attentional modulation throughout the visual system. However, among the cells that are significantly influenced by acetylcholine injection, we did not observe an altered attentional modulation during injection. The observed weak influence is possibly due to the low number of significantly affected cells.

The basal forebrain is considered to be the main cholinergic source, providing all cortical areas with acetylcholine via topographically organized but rather broad innervations with acetylcholine (Bigl et al., 1982; Henderson, 1981; Johnston et al., 1981). In top-down processes such as sustained attention, the cholinergic supply is proposed to be regulated via direct connections from prefrontal areas either to the basal forebrain or to posterior attention systems (Sarter et al., 2000). This regulatory mechanism with its broad cholinergic innervation and slow temporal resolution is challenging to reconcile with the fast local mechanism of spatial attention.

However, an additional intracortical cholinergic source is provided by intrinsic bipolar cholinergic interneurons (Levey et al., 1984; Houser et al., 1985). As their spatial expansion goes along with the columnar orientation of the cortex, 
they are suited to modulate fast attentional effects on small cortical units (von Engelhardt et al., 2007).

Another possible explanation of the cholinergic involvement in attentional modulation is that the coarse release of acetylcholine is locally triggers a noncholinergic mechanism and is therefore indirectly involved in attentional modulation. However, a study performing neuropharmacological manipulations in area $\mathrm{V} 1$ in awake, behaving monkeys, reported an increase in attentional modulation with acetylcholine injection (Herrero et al., 2008), similar to our observation.

\section{Nicotinic receptor contribution in attentional modulation}

As acetylcholine additionally acts on nicotinic receptors, they might also contribute to attentional modulation in area MT. As data collection is ongoing, we presented only sample cells, which were pharmacologically targeted using the general nicotinic antagonist mecamylamine. The effects on the cells' firing rates were ambiguous, showing either an increase or a total shutdown in firing rate during injection. However, the amount of recorded cells is not sufficient to draw strong conclusions.

In general, the nicotinic antagonist mecamylamine is not competitive in its binding abilities and therefore constitutes in that sense a good antagonist. However, it shows voltage dependent binding characteristics and inhibits NMDA receptors at high concentrations (MacLeod et al., 1984, O'Dell and Christensen, 1988). So far only one concentration ( $0.1 \mathrm{~mol} / \mathrm{l})$ has been tested within this study, which is possibly too high, leading to the observed shutdown in firing rate. Further manipulations are needed to investigate the exact involvement in attentional modulation in area MT, but the observed effects on single cell level, may lead to the assumption, that the nicotinic receptor plays a 
modulatory role in attention guided change in firing rate. In area V1, it was shown that the nicotinic receptor type is not involved in attentional modulation.

\section{Behavioral effects}

As we assume to affect only a few hundred micrometers with our injection (Herrero, 2011), we did not expect to observe any change in behavior. Indeed, none of the injected substances had a significant main influence on reaction time. Even when we analyzed only those data files in which the recorded cells showed a significant influence of injection, no effect on reaction time was observed. In contrast, in area V1 a faster reaction time was observed when low doses of acetylcholine were injected (Herrero et al., 2008). Additionally, no linear relationship was found in the current results between injection-induced change in firing rate and task performance, measured as a change in reaction time. The overall lack of injection-induced changes in reaction time suggests that we are indeed only manipulating a very local area in MT and therefore our manipulation is not sufficient to influence a global behavior pattern.

The attentional task (attention inside or outside the receptive field or at fixation point), on the other hand, always showed strong effects on reaction time. The difference is very likely driven by the task difference in attend-in/attend-out vs. attend fixation. We wouldn't expect a difference between attend-in and attendout task condition, because they are essentially the same task for the monkey, whereas responding to a luminance change of the fixation point (attendfixation) is easier and the response of the monkey is therefore faster. 


\section{Selection of substance for a more specific blockage}

The antagonists and agonist used in this study are known for their broad binding characteristics. On the one hand, this broad influence allows the investigation of a general involvement of one receptor type and they are therefore used as standard antagonists in many studies investigating influences on a cellular level as well as in cognitive tasks (Herrero et al., 2008, Aggelopoulos et al., 2011). But on the other hand, the broad blockage of a receptor type could lead to misleading results. Ideally, specific antagonists are additionally used, which only bind to one type of receptor. For example, if a more specific muscarinic antagonist had been used, its use would leave the other muscarinic receptors unrestricted in their binding ability and an unadulterated contribution to attentional modulation could be investigated. Although some specific antagonists have been proposed in the pharmacology literature, most of these specific antagonists are not sufficiently tested in vivo. Of highest interest would be the muscarinic subtype $\mathrm{m} 1$, as it is proposed to be involved in attentional modulation (Disney et al, 2014).

Also for the nicotinic receptor type, various specific receptors are of particular interest. The homomeric alpha7 subunit-containing receptor is a possible mediator of attentional modulation (Disney et al., 2014), but also alpha5 and beta2 are promising candidates (see Proulx et al., 2014 for a review). Heteromeric nicotinic receptors on the other hand are very unlikely to be involved in attentional modulation processes, as they are not strongly expressed in macaque area MT (Disney et al., 2007).

\section{Local cholinergic effects}

Acetylcholine acts as a neuromodulator in the brain and is therefore seen to have limited influence on local and fast attention-mediated effects. An interaction with other neurotransmitter systems is therefore very likely. 
Glutamate is the major excitatory and fast-acting neurotransmitter in the brain (see for a review Meldrun, 2000). It is released from visual cortical neurons when they are visually stimulated (bottom-up process). It is proposed that this bottom-up process interacts with top-down influences such as spatial attention, mediated by cholinergic release. This synergistic effect is therefore proposed to enhance signal detection ( $\mathrm{Yu}$ and Dayan, 2002 and 2005). As well as the neurotransmitter glutamate, other neurotransmitters are also likely to be involved in attentional modulation, such as the inhibitory neurotransmitter $\gamma$ Aminobutyric acid (GABA).

In area MT, we found that spatial attention was increased when muscarinic receptor action was blocked. The following scenarios are possible explanations for this effect. As shown in Figure 22a, due to depolarization of glutamatergic neurons, a release of glutamate is induced. Glutamate binds on AMPA/NMDA receptors, located on the post-synaptic cortical neuron, inducing an increased firing rate. The glutamatergic neuronal endings are equipped with nicotinic cholinergic receptors. If acetylcholine is now additionally available in the synaptic cleft, it binds to the nicotinic receptors and induces a further increase in glutamate release. This leads to a further increase in available glutamate in the synaptic cleft and the firing rate of the postsynaptic cortical neuron is further increased. The cholinergic neuron itself is equipped with a muscarinic receptor (mAChR). Presynaptic mAChRs of subtype $\mathrm{m} 2$ are predominantly found in cortex and are mainly inhibitory, serving as a negative feedback loop in signal transduction (Wess, 2003). If the muscarinic receptor type is now blocked, for example by introducing the antagonist scopolamine, as we did in this experiment, more acetylcholine is released in the synaptic cleft. This in turn results in an increased release of glutamate and therefore in an increased activity of the cortical neuron.

Another possible inhibitory scenario, involving the neurotransmitter GABA, is illustrated in Figure 22b. GABA is released, acting on other cortical neurons, 
inducing a reduction in firing rate. If acetylcholine is now additionally available in the synaptic cleft, it binds to muscarinic receptors - very likely of type $\mathrm{m} 1$ (Disney et al., 2014) - located on GABAergic-cells. This leads to an increased release of GABA, resulting in a reduction of firing rate.

a)

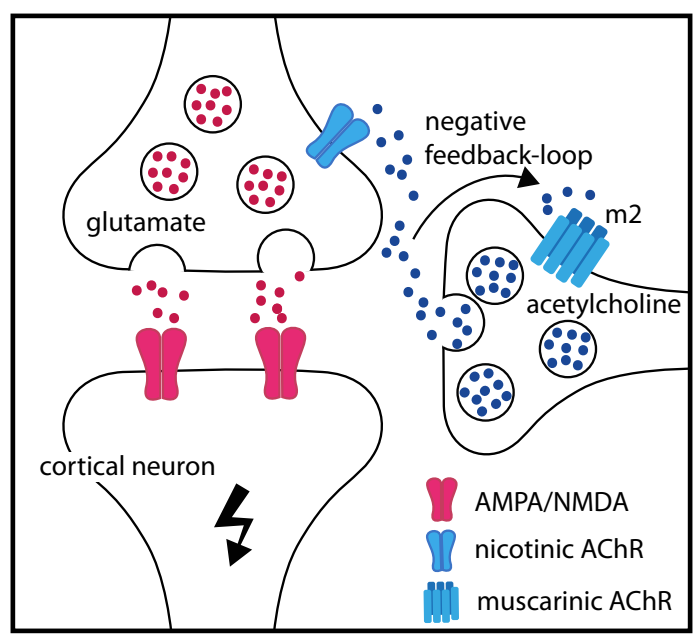

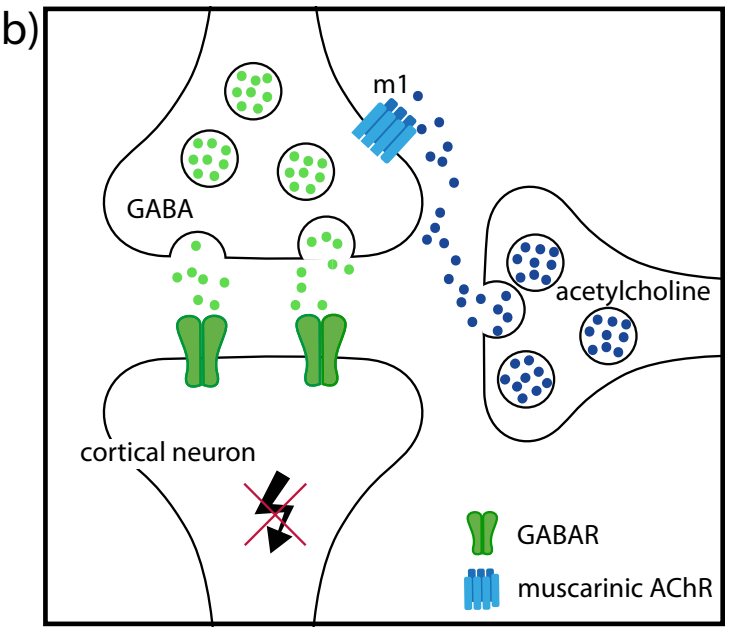

Figure 22. Two possible cholinergic effects having excitatory (a) and inhibitory (b) influence on local cortical circuitry. a) A release of acetylcholine enhances glutamatergic release, via the nicotinic receptor type. Cholinergic release is self-regulated via a negative feedback-loop, mediated by the muscarinic receptor type. A blockage of the muscarinic receptor type increases the release of acetylcholine and therefore mediates further glutamate release, resulting in an increase in firing rate. b) $Y$-Aminobutyric acid (GABA) is released in the synaptic cleft, binding on GABAergic neurons and inducing a decrease in firing rate. A release of acetylcholine induces a further release of GABA, as it binds on muscarinic receptor subtype M1, located on GABAergic cells.

\section{Outlook}

It could be argued that the weak effect of injection observed in this study is due to the marginally larger distance between recording electrode and micropipette (inter-tip distance: $305 \mu \mathrm{m}$ ) compared to other injection set-ups (approx. $30 \mu \mathrm{m}$ in Thiele et al., 2006). In order to exclude this constraint, we plan to measure the effect of our pharmacological manipulations using modified recording equipment, that will diminish the distance between electrode and micropipette tips (approx. $100 \mu \mathrm{m}$ ). Additionally, it is a high priority to continue collecting 
data to further investigate the involvement of nicotinic receptors. Further work could also target specific cholinergic neurotransmitter subtypes in attentional modulation or investigate the interplay with other neurotransmitter systems such as the glutamatergic (Herrero et al., 2013) or the GABAergic system (Disney et al., 2014).

\section{References}

Aggelopoulos NC, Liebe S, Logothetis NK, Rainer G (2011) Cholinergic control of visual categorization in macaques. Front Behav Neurosci. 15:5-73.

Arnold HM, Burk JA, Hodgson EM, Sarter M, Bruno JP (2002) Differential cortical acetylcholine release in rats performing a sustained attention task versus behavioral control tasks that do not explicitly tax attention. Neuroscience 114:451-460.

Bentley P, Vuilleumier P, Thiel CM, Driver J, Dolan RJ (2003) Cholinergic enhancement modulates neural correlates of selective attention and emotional processing. Neuroimage 20(1):58-70.

Bigl V, Woolf NJ, Butcher LL (1982) Cholinergic projections from the basal forebrain to frontal, parietal, temporal, occipital, and cingulate cortices: a combined fluorescent tracer and acetylcholinesterase analysis. Brain Research Bulletin 8(6):727-49.

DeFelipe J, González-Albo MC, Del Río MR, Elston GN (1999) Distribution and patterns of connectivity of interneurons containing calbindin, calretinin, and parvalbumin in visual areas of the occipital and temporal lobes of the macaque monkey. Journal of Comparable Neurology 412(3):515-26.

Disney AA, Domakonda KV, Aoki C (2006) Differential expression of muscarinic acetylcholine receptors across excitatory and inhibitory cells in visual cortical areas V1 and V2 of the macaque monkey. J Comp Neurol 499(1):49-63. 
Disney AA, Aoki C, Hawken MJ (2012) Cholinergic suppression of visual responses in primate $\mathrm{V} 1$ is mediated by GABAergic inhibition. $\mathrm{J}$ Neurophysiol. 108(7):1907-23.

Disney AA, Alasady HA, Reynolds JH (2014) Muscarinic acetylcholine receptors are expressed by most parvalbumin-immunoreactive neurons in area MT of the macaque. Brain Behav. 4(3):431-45.

Disney AA, Reynolds JH (2014) Expression of m1-type muscarinic acetylcholine receptors by parvalbumin-immunoreactive neurons in the primary visual cortex: a comparative study of rat, guinea pig, ferret, macaque, and human. J Comp Neurol. 522(5):986-1003.

Fournier GN, Semba K, Rasmusson DD (2004) Modality and region specific acetylcholine release in the rat neocortex. Neuroscience 126:257-262.

Henderson Z (1981) A projection from acetylcholinesterase-containing neurones in the diagonal band to the occipital cortex of the rat. Neuroscience 6(6):1081-1088.

Herrero JL, Roberts MJ, Delicato LS, Gieselmann MA, Dayan P, Thiele A (2008) Acetylcholine contributes through muscarinic receptors to attentional modulation in V1. Nature 454(7208):1110-4.

Herrero JL (2011) Neurophysiology and neuropharmacology of visual attention. Thesis

Herrero JL, Gieselmann MA, Sanayei M, Thiele A (2013) Attention-induced variance and noise correlation reduction in macaque $\mathrm{V} 1$ is mediated by NMDA receptors. Neuron 78(4):729-39.

Houser CR, Crawford GD, Salvaterra PM, Vaughn JE (1985) Immunocytochemical localization of choline acetyltransferase in rat cerebral cortex: a study of cholinergic neurons and synapses. J Comp Neurol 234:17-34. 
Johnston MV, McKinney M, Coyle JT (1981) Neocortical cholinergic innervation: a description of extrinsic and intrinsic components in the rat. Experimental Brain Research 43(2):159-172.

Klinkenberg I, Sambeth A, Blokland A (2011) Acetylcholine and attention. Behav Brain Res. 221(2):430-42.

Kumari V, Gray JA, Ffytche DH, Mitterschiffthaler MT, Das M, Zachariah E, Vythelingum GN, Williams SCR, Simmons A, Sharma T (2003) Cognitive effects of nicotine in humans: an fMRI study. Neuroimage 19:1002-1013.

Levey IA, Wainer BH, Rye DB, Mufson EJ, Mesulam MM (1984) Choline acetyltransferase-immunoreactive neurons intrinsic to rodent cortex and distinction from acetylcholinesterase-positive neurons. Neuroscience 13(2): 341-353.

Maunsell JHR, Cook EP (2002) The role of attention in visual processing. Philos. Trans. R. Soc. London Ser. B 357:1063-1072.

McGaughy J, Dalley JW, Morrison CH, Everitt BJ, Robbins TW (2002) Selective behavioral and neurochemical effects of cholinergic lesions produced by intrabasalis infusions of 192 IgG- saporin on attentional performance in a five-choice serial reaction time task. Journal of Neuroscience 22:19051913.

MacLeod NK, James TA, Starr MS (1984) Muscarinic action of acetylcholine in the rat ventromedial thalamic nucleus. Exp Brain Res. 55(3):553-61.

Meldrum BS (2000) Glutamate as a neurotransmitter in the brain: review of physiology and pathology. J Nutr. 130(4S Suppl):1007S-15S.

Mesulam MM, Mufson EJ, Levey AI, Wainer BH (1983b). Cholinergic innervation of cortex by the basal forebrain: cytochemistry and cortical connections of the septal area, diagonal band nuclei, nucleus basalis (substantia innominata), and hypothalamus in the rhesus monkey. Journal of Comparative Neurology 214:170-197. 
O'Dell TJ, Christensen BN (1988) Mecamylamine is a selective non-competitive antagonist of N-methyl-D-aspartate- and aspartate-induced currents in horizontal cells dissociated from the catfish retina. Neurosci Lett. 94(12):93-8.

Ohayon S, Tsao DY (2012) MR-guided stereotactic navigation. J Neurosci Methods. 204(2):389-97.

Parikh V, Sarter M (2008) Cholinergic mediation of attention: contributions of phasic and tonic increases in prefrontal cholinergic activity. Ann N Y Acad Sci. 1129:225-35.

Reynolds JH, Chelazzi L, Desimone R (1999) Competitive mechanisms subserve attention in macaque areas V2 and V4. J Neurosci 19:1736-1753.

Roelfsema PR, Lamme VA, Spekreijse H (1998) Object-based attention in the primary visual cortex of the macaque monkey. Nature 395:376:381.

Proulx E, Piva M, Tian MK, Bailey CD, Lambe EK (2014) Nicotinic acetylcholine receptors in attention circuitry: the role of layer VI neurons of prefrontal cortex. Cell Mol Life Sci. 71(7):1225-44.

Sarter M, Hasselmo ME, Bruno JP, Givens B (2005) Unraveling the attentional functions of cortical cholinergic inputs: interactions between signaldriven and cognitive modulation of signal detection. Brain Res Brain Res Rev. 48(1):98-111.

Thiele A, Herrero JL, Distler C, Hoffmann KP (2012) Contribution of cholinergic and GABAergic mechanisms to direction tuning, discriminability, response reliability, and neuronal rate correlations in macaque middle temporal area. Journal of Neuroscience 32(47):1660215 .

Treue S, Maunsell JH (1996) Attentional modulation of visual motion processing in cortical areas MT and MST. Nature 382(6591):539-41. 
Treue S, Maunsell JH (1999) Effects of attention on the processing of motion in macaque middle temporal and medial superior temporal visual cortical areas. The Journal of Neuroscience 19(17):7591-602.

von Engelhardt J, Eliava M, Meyer AH, Rozov A, Monyer H (2007) Functional characterization of intrinsic cholinergic interneurons in the cortex. The Journal of Neuroscience 27(21):5633-5642.

Wess J (2003) Novel insights into muscarinic acetylcholine receptor function using gene targeting technology. Trends Pharmacol Sci. 24(8):414-20.

Wevers A, Burghaus L, Moser N, Witter B, Steinlein OK, Schütz U, Achnitz B, Krempel U, Nowacki S, Pilz K, Stoodt J, Lindstrom J, De Vos RA, Jansen Steur EN, Schröder H (2000) Expression of nicotinic acetylcholine receptors in Alzheimer's disease: postmortem investigations and experimental approaches. Behav Brain Res. 113(1-2):207-15.

Yu AJ, Dayan P (2002) Acetylcholine in cortical inference. Neural Netw. 15(46):719-30.

Yu AJ, Dayan P (2005) Uncertainty, neuromodulation, and attention. Neuron 46(4):681-92. 
Supplementary figures:

a) Inclusion criterion 1: trial repetitions
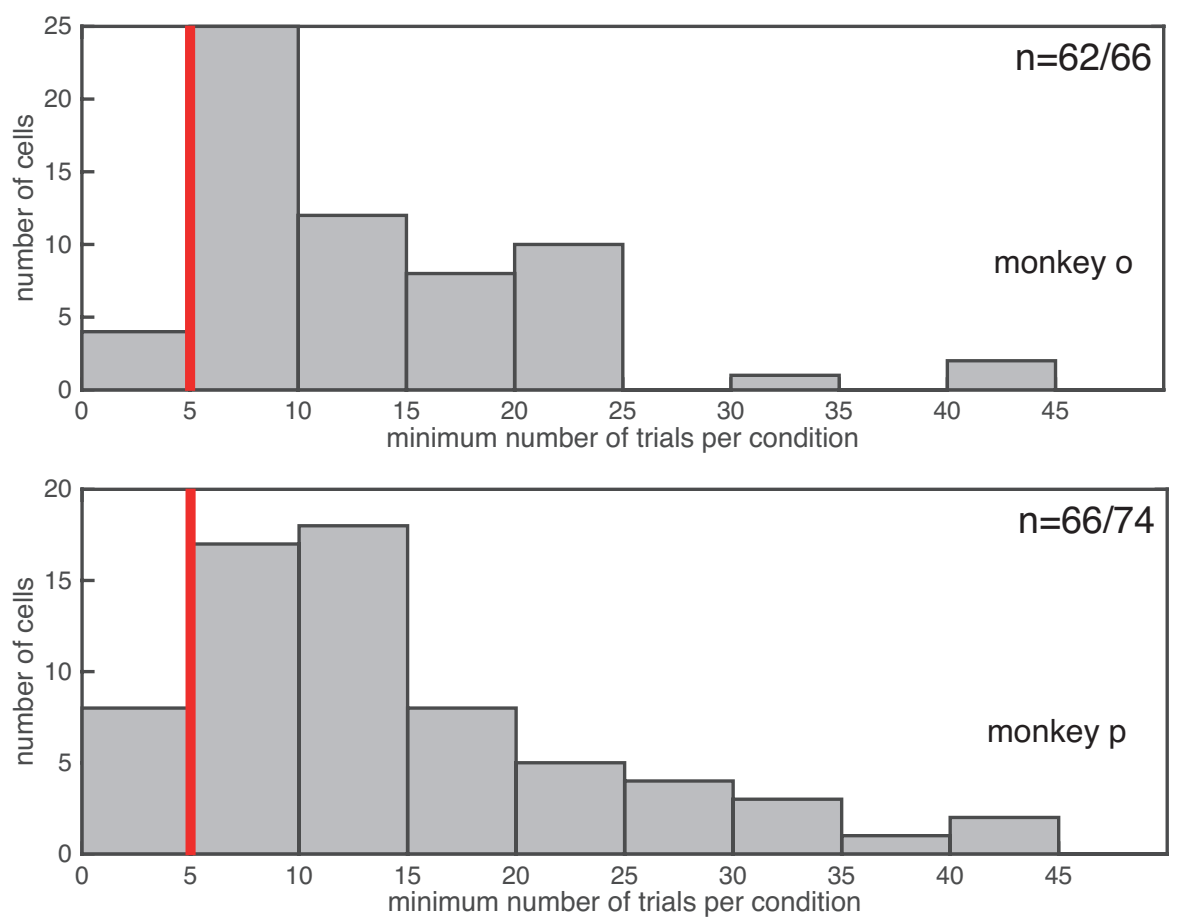

b) Inclusion criterion 2: firing rate
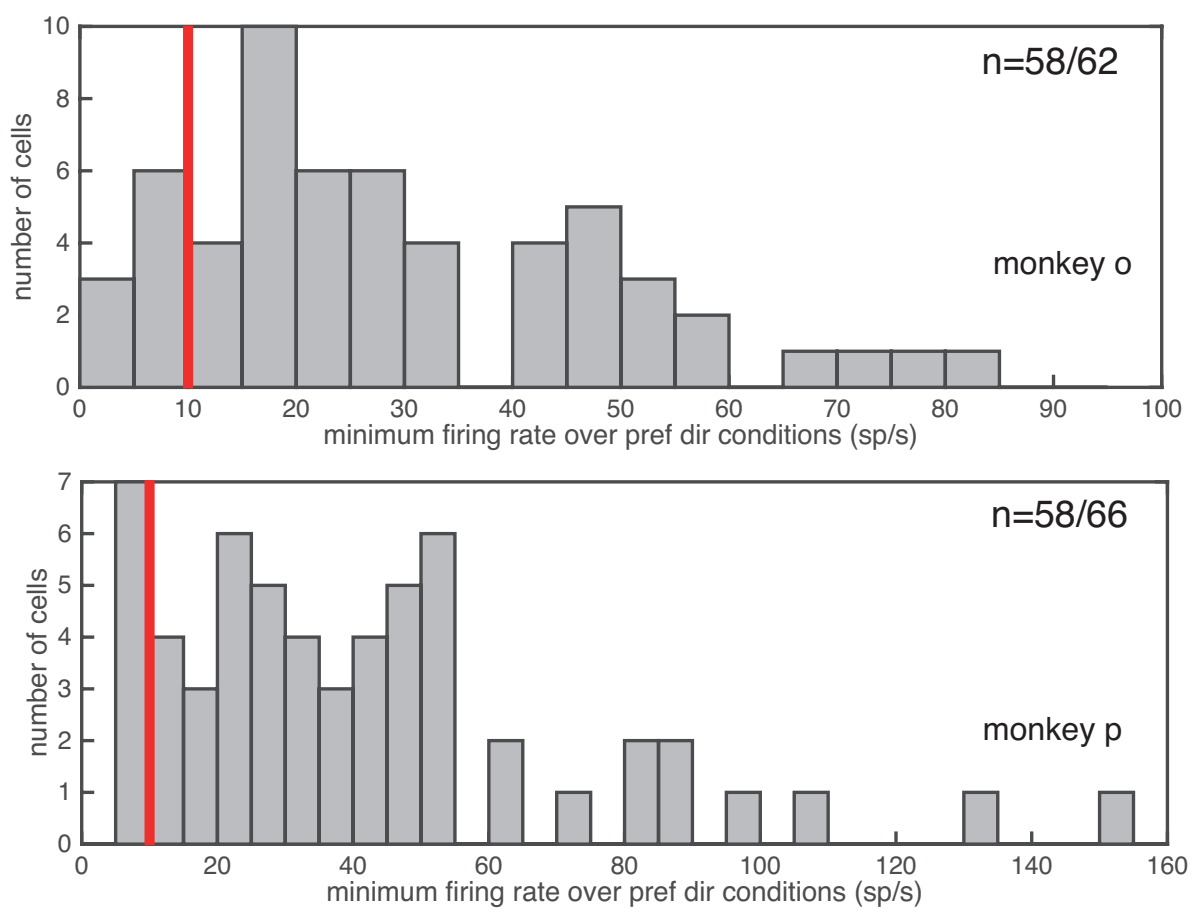
c) Inclusion criterion 3: responsiveness
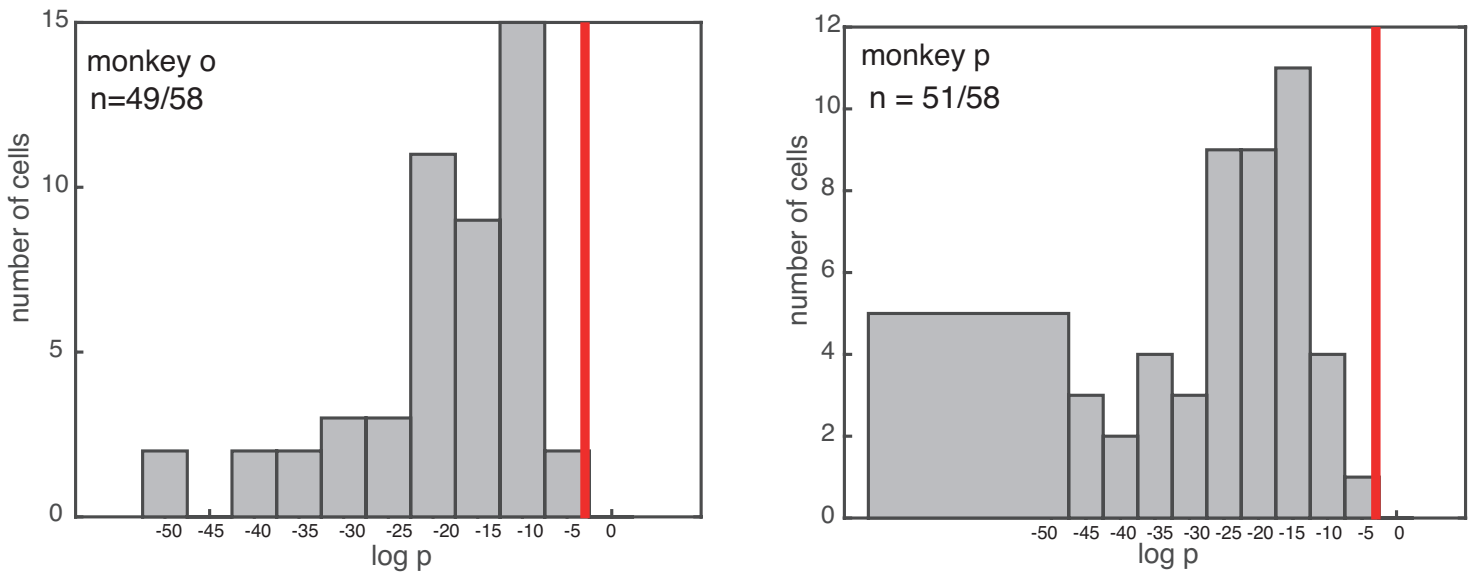

Sup.Fig.1: Histograms of the included cells for the first inclusion criteria for the data recorded with scopolamine injection for monkey o (left column) and monkey p (right column). Red vertical bars indicate the threshold for data inclusion. a) Data files were included, when they contained a minimum of 5 repetitions of every task condition. b) The isolated cells had to fire with a minimum firing rate of 10 spikes/second to be included in further analysis. c) A responsiveness check was performed comparing the sensory conditions for preferred and null direction during the control block. Cells were included in further analysis showing significant differences between direction conditions (Kruskal-Wallis test, $p=0.05$ ). 
Acetylcholine

\begin{tabular}{|c|c|c|c|}
\hline 2 & 0.1 & 0.15 & 0.2 \\
\hline 3 & 6 & 0 & 0 \\
\hline 4 & 11 & 14 & 3 \\
\hline
\end{tabular}

Scopolamine

\begin{tabular}{|c|c|c|c|}
\hline 1 & 3 & 1 & 16 \\
\hline 2 & 6 & 48 & 33 \\
\hline 4 & 0 & 6 & 23 \\
\hline
\end{tabular}

Saline

\begin{tabular}{|c|c|}
\hline 2 & 31 \\
\hline vol conc & 0.1 \\
\hline 4 & 7 \\
\hline 6 & 1 \\
\hline
\end{tabular}

Sup.Fig.2: Overview of the number of recording sessions for every substance injected. The $\mathrm{x}$-axis depicts the various concentrations used (mol/l), whereas the $\mathrm{y}$-axis depicts the different volumes (nl) injected within one minute. Mecamylamine was only injected with a concentration of $0.1 \mathrm{~mol} / \mathrm{l}$ and an injection rate of $6 \mathrm{nl} /$ minute and is therefore not presented in a table. 

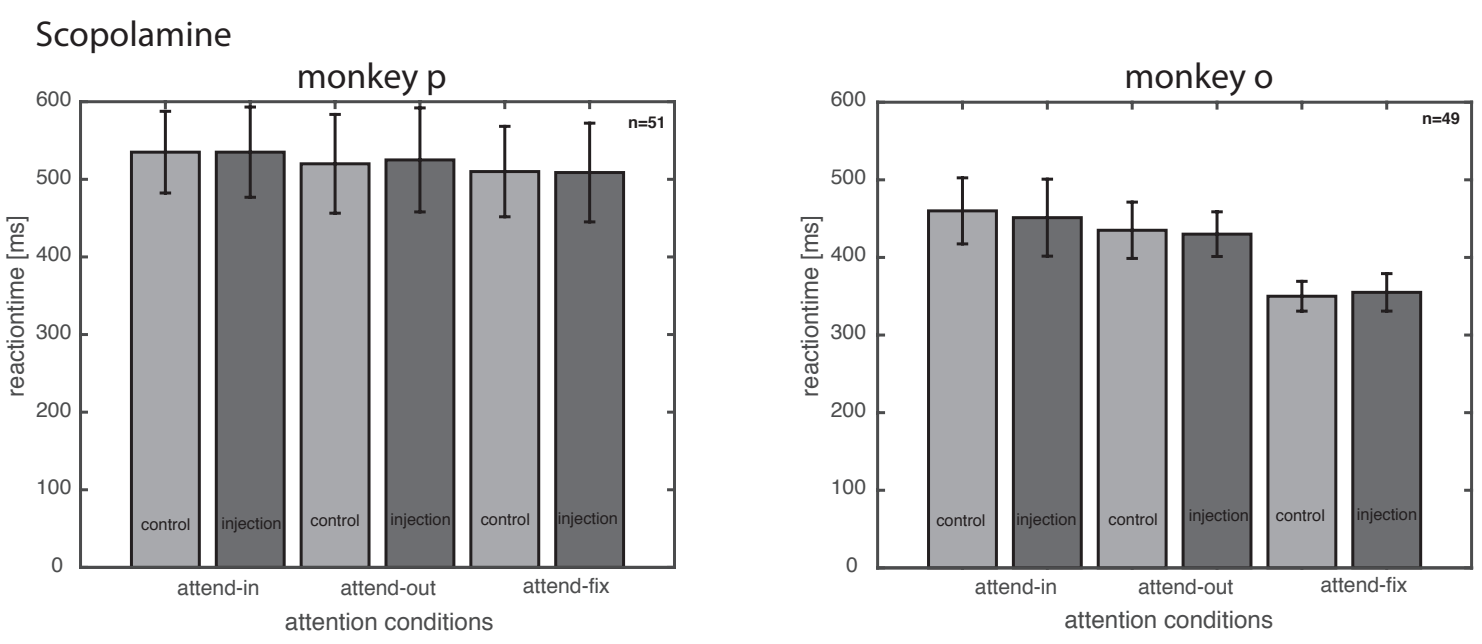

Acetylcholine
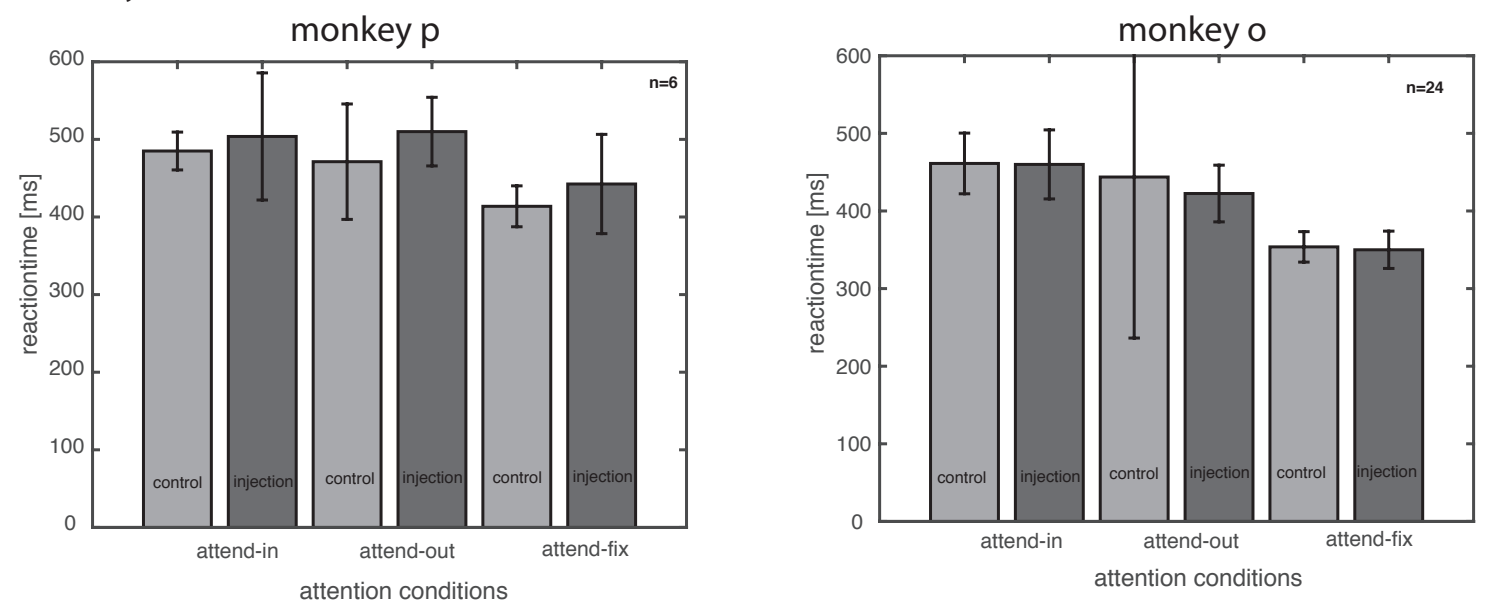

Saline
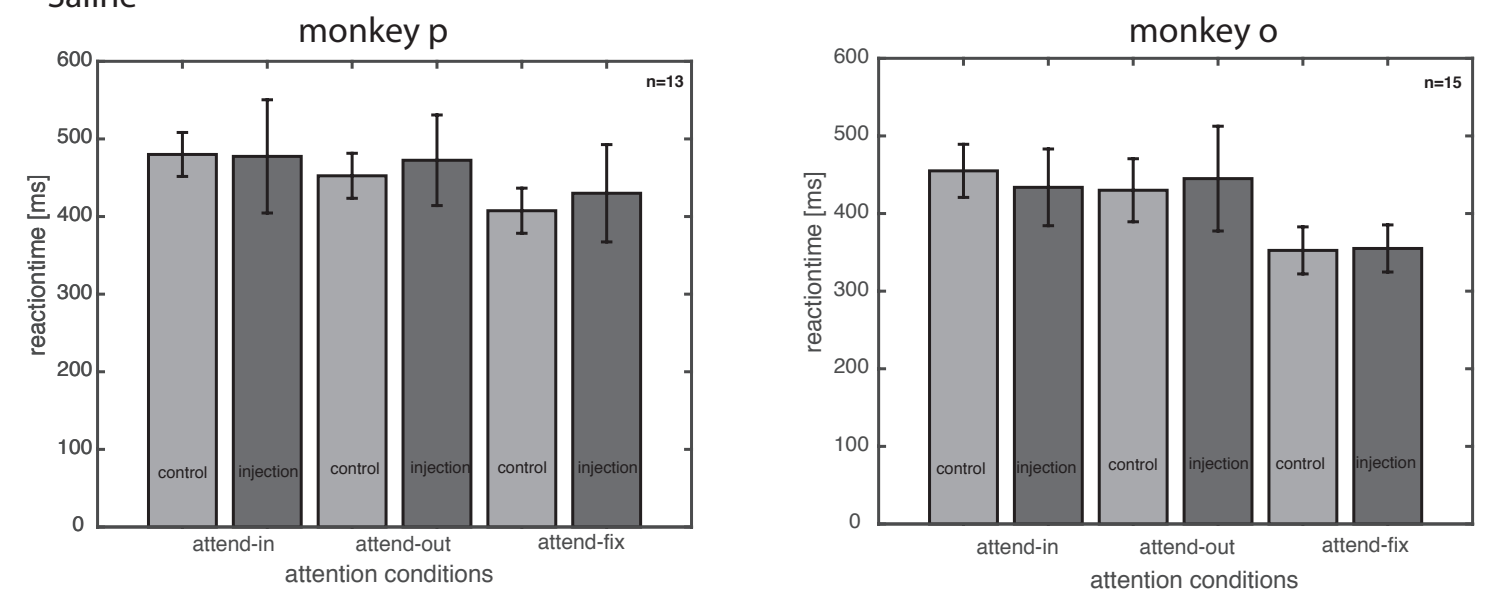
Sup.Fig.3: Median reaction times for the three attention conditions and three substances. Median firing rates during the control block (light grey), where no substance was injected, were compared to the injection block (dark grey). Either the muscarinic antagonist scopolamine (first row), or the agonist acetylcholine (middle row), or the control substance saline (lower row) was injected. For each substance used, three spatial attention conditions (attention inside the receptive field (attend-in), attention outside of the receptive field (attendout) or attention at the fixation point (attend-fix)) were contrasted. Error bars show standard deviation.

\section{Supplementary tables:}

The tables incorporate information about the recorded data files when injection was performed. Tables are shown separated by monkey and substance injected. Abbreviations of the column descriptions are explained below:

no. - number of the recorded file

date - recording date

filename - filename of the recorded data

eparUsed - name of the experimental script used generating the stimuli

mclabUnitNo - channel number used within our recording software; lfp = local field potentials, $\mathrm{mu}=$ multiunit activity

electrodeNo - the number of the electrode used to record the data

depth - depth of the recording electrode and micropipette tip in mm when data streaming was started

noDirectionsUsed - number of linear movement directions used; 2= preferred direction and null direction

stim radius - radius of the stiumulus (random dot pattern)

prefDir - preferred direction to linear movement of the isolated main single unit

xStimulus - x position of the stimulus within the neuron's receptive field on the screen (deg) 
yStimulus - y position of the stimulus within the neuron's receptive field on the screen (deg)

degreeOfResponseEvent - direction change of the stimulus (deg), the monkey has to respond to

substanceInjected - substance used in this data file; $\mathrm{sco}=$ scopolamine, $\mathrm{ACh}=$ acetylcholine, $\mathrm{MM}=$ mecamylamine, $\mathrm{NaCl}=$ saline

concentration - concentration of substance used in mol/l

amountOfCycles - the number of injection blocks

injectionrate - the amount of $\mathrm{nl}$ injected every minute

trialBegin1 - trial number when the first injection was performed within the first injection block; 2 and 3 indicate the first injection of the second and the third injection block

trialEnd1 - trial number one minute after the last injection of the first injection block; 2 and 3 indicate the second and the third injection block

plexStart1 - the time in the Plexon data file when the first injection was performed within the first injection block; 2 and 3 indicate the second and the third injection block

plexEnd1 - the time in the Plexon data file one minute after the last injection was performed in the first injection block; 2 and 3 indicate the second and the third injection block

cut after or between trial num - trial number of the experiment after which the isolation of the recorded cell is not trustworthy. Data file needs to be cut after this trial. When two trial numbers are shown, the data file needs to be cut between those trials. 


\begin{tabular}{|c|c|c|c|c|}
\hline no. & date & filename & eparUsed & mclabUnitNo \\
\hline 1 & 15.04 .15 & vecl-spANP2-osk-077-01+01 & 15-Apr-2015a & 1 \\
\hline 2 & 16.04.15 & vecl-spANP2-osk-078-01+01 & 16-Apr-2015a & Ifp \\
\hline 3 & 21.04.15 & vecl-spANP2-osk-080-01+01 & 21-Apr-2015a & $1, \mathrm{mu}$ \\
\hline 4 & 23.04 .15 & vecl-spANP2-osk-082-01+01 & 23-Apr-2015a & 2 \\
\hline 5 & 24.04 .15 & vecl-spANP2-osk-083-01+01 & 24-Apr-2015a & 2 \\
\hline 6 & 28.04 .15 & vecl-spANP2-osk-084-01+01 & 28-Apr-2015a & 1 \\
\hline 7 & 29.04 .15 & vecl-spANP2-osk-085-01+01 & 29-Apr-2015a & 1 \\
\hline 8 & 30.04 .15 & vecl-spANP2-osk-086-01+01 & 30-Apr-2015a & 1 \\
\hline 9 & 06.05 .15 & vecl-spANP2-osk-088-01+01 & 06-May-2015a & 1,2 \\
\hline 10 & 12.05 .15 & vecl-spANP2-osk-089-01+01 & 12-May-2015 & Ifp \\
\hline 11 & 07.10 .15 & vecl-spANP-osk-090-01+01 & 07-Oct-2015-a & 2,10 \\
\hline 12 & 09.10 .15 & vecl-spANP-osk-091-01+01 & 09-Oct-2015-a & 2 \\
\hline 13 & 13.10 .15 & vecl-spANP-osk-092-01+01 & 13-Oct-2015-a & 2 \\
\hline 14 & 14.10 .15 & vecl-spANP-osk-093-01+01 & 14-Oct-2015-a & $\mathrm{mu}$ \\
\hline 15 & 15.10 .15 & vecl-spANP-osk-094-01+01 & 15-Oct-2015a & 1 \\
\hline 16 & 16.10 .15 & vecl-spANP-osk-095-01+01 & 16-Oct-2015a & 2,10 \\
\hline 17 & 22.10 .15 & vecl-spANP-osk-096-01+01 & 22-Oct-2015a & 1a, MU \\
\hline 18 & 30.10 .15 & vecl-spANP-osk-098-01+02 & 30-Oct-2015a & offlineSort \\
\hline 19 & 06.11 .15 & vecl-spANP-osk-100-02+01 & 06-Nov-2015a & offlineSort \\
\hline 20 & 12.11 .15 & vecl-spANP-osk-102-01+01 & 12-Nov-2015a & $1 \mathrm{a}$ \\
\hline 21 & 25.11 .15 & vecl-spANP-osk-104-01+01 & 25-Nov-2015a & offlinesort $2 a, 2 b$ \\
\hline 22 & 01.12 .15 & vecl-spANP-osk-106-01+01 & 01-Dec-2015-a & 1a, 2a(mua) \\
\hline 23 & 02.12 .15 & vecl-spANP-osk-107-01+02 & 02-Dec-2015b & $1 \mathrm{a}$ \\
\hline 24 & 02.12 .15 & vecl-spANP-osk-107-02+01 & 02-Dec-2015b & $1 \mathrm{a}$ \\
\hline 25 & 03.12 .15 & vecl-spANP-osk-108-01+01 & 03-Dec-2015b & Ifp \\
\hline 26 & 08.12 .15 & vecl-spANP-osk-109-01+01 & 08-Dec-2015b & $1 \mathrm{a}$ (offline sorting) \\
\hline 27 & 08.12 .15 & vecl-spANP-osk-109-02+01 & 08-Dec-2015b & $1 \mathrm{a}$ (offline sorting) \\
\hline 28 & 09.12 .15 & vecl-spANP-osk-110-01+01 & 09-Dec-2015b & $1 \mathrm{a}$ (offline sorting) \\
\hline 29 & 15.12 .15 & vecl-spANP-osk-112-01+01 & 15-Dec-2015b & 1,9 \\
\hline 30 & 16.12 .15 & vecl-spANP-osk-113-01+01 & 16-Dec-2015a & 1,9 (msu) \\
\hline 31 & 17.12.15 & vecl-spANP-osk-114-01+01 & 17-Dec-2015a & 1 \\
\hline 32 & 18.12 .15 & vecl-spANP-osk-115-01+01 & 18-Dec-2015a & 1,9 \\
\hline 33 & 19.12.15 & vecl-spANP-osk-116-01+01 & 19-Dec-2015a & 1 (offlinesorting) \\
\hline 34 & 20.12 .15 & vecl-spANP-osk-117-01+01 & 20-Dec-2015a & 1 \\
\hline
\end{tabular}

Acetylcholine monkey P

$\begin{array}{rllrr}\text { no. } & \text { date } & \text { filename } & \text { eparUsed } & \text { mclabUnitNo } \\ 1 & 29.01 .14 & \text { vecl-spANP2-pie-082-01+01 } & \text { 24Jan-b } & 1,2 \\ 2 & 30.01 .14 & \text { vecl-spANP2-pie-083-02+01 } & \text { 30Jan-a } & 1 \\ 3 & 05.02 .14 & \text { vecl-spANP2-pie-085-01+02 } & \text { 5Feb-a } & 2 \\ 4 & 06.02 .14 & \text { vecl-spANP2-pie-086-04+01 } & 6 \text { Feb-a } & 1 \\ 5 & 12.02 .14 & \text { vecl-spANP2-pie-089-02+01 } & \text { 12Feb-a } & 1 \\ 6 & 13.02 .14 & \text { vecl-spANP2-pie-090-01+02 } & \text { 13Feb-a } & 2\end{array}$


Acetylcholine monkey 0

\begin{tabular}{|c|c|c|c|c|c|c|c|c|c|}
\hline no. & Plexon_SU_number & electrodeNo & depth & noDirectionsUsed & stim radius & prefDir & prefSpeed & xStimulus & yStimulus \\
\hline 1 & $1 a, 2 a$ & 1 & 4719 & 2 & 2,4 & 180 & 12 & $-11,4$ & 7,9 \\
\hline 2 & & 1,2 & 4000 & 2 & 2 & 90 & 12 & -12 & 7,9 \\
\hline 3 & & 1 & 5500 & 2 & 2 & 210 & 12 & $-2,4$ & 6,2 \\
\hline 4 & $2 a$ & 2 & 3285 & 2 & 2,2 & 180 & 12 & -12 & 6,2 \\
\hline 5 & $1 \mathrm{a}, 2 \mathrm{a}$ & 2 & 3324 & 2 & 2,4 & 150 & 12 & -15 & 8 \\
\hline 6 & $1 a, 2 a$ & 1 & 3809 & 2 & 2 & 0 & 8 & $-12,2$ & 7,5 \\
\hline 7 & $1 a, 2 a$ & 1 & 3163 & 2 & 2 & 150 & 8 & $-12,4$ & 1,5 \\
\hline 8 & $1 a$ & 1 & 5021 & 2 & 2 & 270 & 8 & $-2,3$ & 7,6 \\
\hline 9 & $1 a, 2 a$ & 1,2 & 4361 & 2 & 2 & 330 & 12 & $-8,4$ & 6,7 \\
\hline 10 & & 1,2 & 5387 & 2 & 2 & 60 & 12 & $-4,9$ & 6,8 \\
\hline 11 & $2 a, 2 b, 2 c$ & 2 & 6990 & 2 & 2 & 130 & 2 & 8,5 & 3,5 \\
\hline 12 & $2 a$ & 2 & 6129 & 2 & 2,1 & 180 & 4 & 9,5 & 4,5 \\
\hline 13 & $2 a$ & 2 & 7421 & 2 & 2,5 & 200 & 12 & 9,6 & 3,6 \\
\hline 14 & $1 a, 1 b, 2 a$ & 1,2 & 8535 & 2 & 2,5 & 120 & 12 & 14 & 3,5 \\
\hline 15 & $1 a$ & 1 & 8496 & 2 & & & & & \\
\hline 16 & $2 a, 2 b$ & 2 & 8538 & 2 & 2,5 & 130 & 6 & 10,7 & 3,6 \\
\hline 17 & $1 a$ & 1,2 & 6935 & 2 & 2 & 235 & 12 & 9,9 & 7,8 \\
\hline 18 & $1 a, 2 a$ & 1,2 & 5212 & 2 & & & & & \\
\hline 19 & $1 a, 2 a$ & 1,2 & 4896 & 2 & & & & & \\
\hline 20 & $1 a$ & 1 & 6877 & 2 & 3 & 300 & 8 & 14,6 & $-0,9$ \\
\hline 21 & $2 c$ & 2 & 6094 & 2 & 2,5 & 170 & 12 & 10,6 & 9,2 \\
\hline 22 & $1 a, 2 a$ & 1,2 & 6766 & 2 & 2,5 & 85 & 12 & 11,9 & 7,4 \\
\hline 23 & $1 a, 1 b$ & 1 & 8674 & 2 & 2,60 & 0 & 6 & 14,4 & 2,7 \\
\hline 24 & $1 a$ & 1 & 8366 & 2 & 2,60 & 0 & 6 & 14,4 & 2,7 \\
\hline 25 & & 1,2 & 6500 & 2 & 2,5 & 0 & 6 & 12,5 & $-0,4$ \\
\hline 26 & $1 \mathrm{a}, 1 \mathrm{~b}$ & 1 & 8455 & 2 & 2,5 & 250 & 12 & 13,2 & 7,1 \\
\hline 27 & $1 a$ & 1 & 8615 & 2 & 2,5 & 250 & 12 & 13,2 & 7,1 \\
\hline 28 & $1 a$ & 1 & 8009 & 2 & 2 & 180 & 8 & 10,5 & 6,3 \\
\hline 29 & $1 a$ & 1 & 8689 & 2 & 2,5 & 170 & 12 & 13,9 & 5,1 \\
\hline 30 & $1 a, 1 b$ & 1 & 6983 & 2 & 2,5 & 30 & 8 & 13,5 & 6 \\
\hline 31 & $1 a$ & 1 & 8058 & 2 & 2,6 & 245 & 8 & 16,1 & 5,8 \\
\hline 32 & $1 a$ & 1 & 8044 & 2 & 2,5 & 280 & 12 & 14,7 & 5,7 \\
\hline 33 & $1 \mathrm{a}, 1 \mathrm{~b}$ & 1 & 5753 & 2 & 2 & 30 & 8 & 9,5 & 6,8 \\
\hline 34 & $1 a$ & 1 & 6128 & 2 & 2 & 270 & 8 & 8,4 & 7,2 \\
\hline
\end{tabular}

Acetylcholine monkey P

\begin{tabular}{|c|c|c|c|c|c|c|c|c|c|}
\hline no. & Plexon_SU_number & electrodeNo & depth & noDirectionsUsed & stim radius & prefDir & prefSpeed & xStimulus & yStimulus \\
\hline 1 & $1 a, 2 a$ & 1,2 & 6816 & 2 & 2 & 60 & 12 & $-1,1$ & $-5,3$ \\
\hline 2 & $1 \mathrm{a}$ & 1 & 2456 & 2 & 2,5 & 330 & 12 & $-2,5$ & $-4,8$ \\
\hline 3 & $1 a, 1 b, 2 a$ & 2 & 5800 & 2 & 2 & 60 & 12 & $-3,9$ & $-3,3$ \\
\hline 4 & $1 a, 1 b$ & 1 & 5700 & 2 & 2 & 250 & 12 & $-2,8$ & -5 \\
\hline 5 & $1 a, 2 a, 2 b$ & 1 & 5221 & 2 & 2 & 30 & 8 & $-3,6$ & $-4,7$ \\
\hline 6 & $2 a, 2 b$ & 2 & 6964 & 2 & 2,5 & 25 & 12 & -8 & 0 \\
\hline
\end{tabular}


Acetylcholine monkey 0

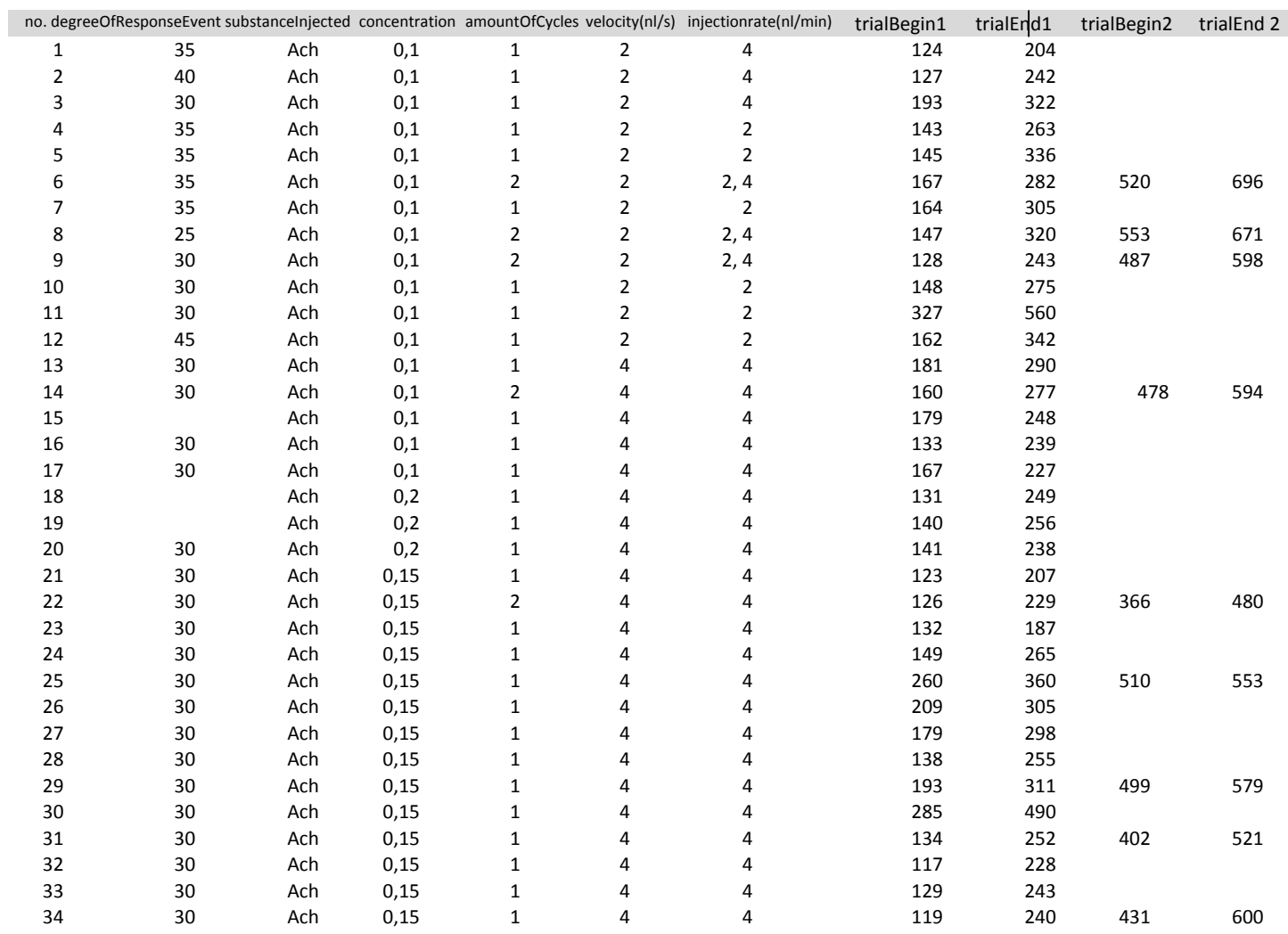

Acetylcholine monkey $\mathrm{P}$

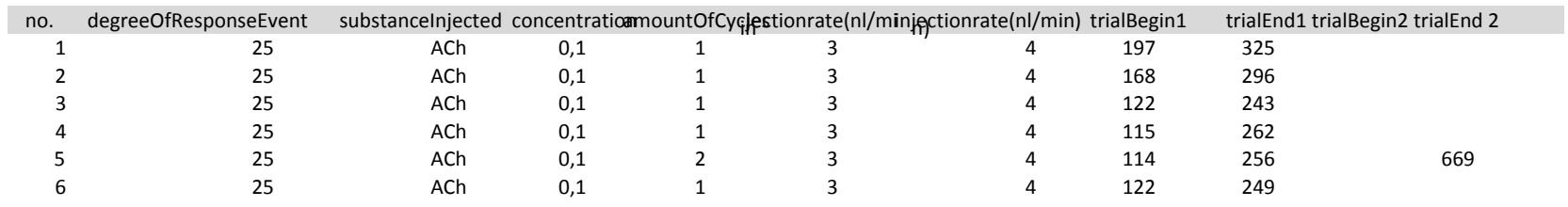


Acetylcholine monkey 0

\begin{tabular}{|c|c|c|c|c|c|c|c|c|c|}
\hline no. & trialBegin3 & trialEnd 3 & plexStart1 & plexEnd1 & plexStart2 & plexEnd2 & plexStart3 & plexEnd 3 & cut after or between trail num \\
\hline 1 & & & $00: 11: 00$ & $00: 18: 00$ & & & & & \\
\hline 2 & & & $00: 12: 00$ & $00: 22: 00$ & & & & & \\
\hline 3 & & & $00: 17: 00$ & $00: 27: 00$ & & & & & \\
\hline 4 & & & $00: 13: 00$ & $00: 23: 00$ & & & & & \\
\hline 5 & & & $00: 13: 00$ & 00:33:00 & & & & & $330,370-380$ \\
\hline 6 & & & $00: 15: 00$ & $00: 25: 00$ & $00: 45: 00$ & 01:00:00 & & & \\
\hline 7 & & & $00: 14: 00$ & $00: 27: 00$ & & & & & $241-268,285-291$ \\
\hline 8 & & & $00: 13: 00$ & $00: 28: 00$ & 00:48:00 & 00:58:00 & & & \\
\hline 9 & & & 00:11:00 & $00: 21: 00$ & 00:44:00 & 00:54:00 & & & \\
\hline 10 & & & $00: 13: 00$ & $00: 23: 00$ & & & & & \\
\hline 11 & & & $00: 34: 00$ & $00: 54: 00$ & & & & & \\
\hline 12 & & & 00:15:00 & 00:30:00 & & & & & \\
\hline 13 & & & 00:19:00 & 00:29:00 & & & & & \\
\hline 14 & & & $00: 14: 00$ & $00: 24: 00$ & $00: 44: 00$ & 00:54:00 & & & \\
\hline 15 & & & $00: 17: 00$ & $00: 27: 00$ & & & & & \\
\hline 16 & & & $00: 12: 00$ & $00: 22: 00$ & & & & & \\
\hline 17 & & & $00: 15: 00$ & $00: 25: 00$ & & & & & \\
\hline 18 & & & $00: 12: 00$ & $00: 22: 00$ & & & & & \\
\hline 19 & & & $00: 12: 00$ & $00: 22: 00$ & & & & & \\
\hline 20 & & & $00: 13: 00$ & $00: 23: 00$ & & & & & \\
\hline 21 & & & $00: 11: 00$ & $00: 20: 00$ & & & & & \\
\hline 22 & & & $00: 11: 00$ & $00: 21: 00$ & $00: 36: 00$ & $00: 46: 00$ & & & \\
\hline 23 & & & $00: 12: 00$ & $00: 18: 30$ & & & & & \\
\hline 24 & & & $00: 18: 00$ & $00: 28: 00$ & & & & & \\
\hline 25 & & & $00: 23: 00$ & $00: 33: 00$ & $00: 48: 30$ & $00: 58: 30$ & & & \\
\hline 26 & & & $00: 20: 00$ & 00:30:00 & & & & & \\
\hline 27 & & & $00: 18: 00$ & $00: 28: 00$ & & & & & \\
\hline 28 & & & $00: 14: 00$ & $00: 24: 00$ & & & & & \\
\hline 29 & & & 00:19:00 & 00:29:00 & 00:45:00 & $00: 56: 30$ & & & \\
\hline 30 & & & $00: 25: 00$ & $00: 45: 00$ & & & & & \\
\hline 31 & 687 & 786 & $00: 13: 00$ & $00: 23: 00$ & 00:38:00 & $00: 48: 00$ & 01:03:00 & 01:13:00 & \\
\hline 32 & & & $00: 10: 00$ & $00: 20: 00$ & & & & & \\
\hline 33 & & & $00: 12: 00$ & $00: 22: 00$ & & & & & \\
\hline 34 & 746 & 865 & $00: 10: 00$ & $00: 20: 00$ & $00: 35: 00$ & 00:50:00 & 01:05:00 & 01:15:00 & \\
\hline
\end{tabular}

Acetylcholine monkey $\mathrm{P}$

\begin{tabular}{|c|c|c|c|c|c|c|c|}
\hline no. & trialBegin3 trialEnd 3 & plexStart1 & plexEnd1 & plexStart2 & plexEnd2 & plexStart3 plexEnd 3 & cut after or between trail num \\
\hline 1 & & $00: 16: 00$ & $00: 26: 00$ & & & & \\
\hline 2 & & 00:13:00 & $00: 23: 00$ & & & & \\
\hline 3 & & $00: 10: 00$ & $00: 20: 00$ & & & & \\
\hline 4 & & 00:09:00 & $00: 20: 00$ & & & & \\
\hline 5 & & 00:09:00 & $00: 20: 00$ & $00: 42: 00$ & $00: 52: 00$ & & \\
\hline 6 & & $00: 10: 00$ & $00: 20: 00$ & & & & \\
\hline
\end{tabular}




\begin{tabular}{|c|c|c|c|c|c|c|}
\hline no. & date & filename & eparUsed & mclabUnitNo & electrodeNo & depth \\
\hline 1 & 17.10 .14 & vecl-spANP2-osk-009-02+01 & 17-Oct-2014-a & 1 & 1 & 2545 \\
\hline 2 & 22.10 .14 & vecl-spANP2-osk-010-02+01 & 22-Oct-2014-a & 2 & 2 & 4109 \\
\hline 3 & 29.10 .14 & vecl-spANP2-osk-013-03+02 & 29-oct-2014a & $1,2, \mathrm{mu}$ & 1,2 & 4091 \\
\hline 4 & 30.10 .14 & vecl-spANP2-osk-014-03+01 & 30-Oct-2014-a & 1,2 & 1,2 & 3853 \\
\hline 5 & 31.10 .14 & vecl-spANP2-osk-015-01+01 & 31-Oct-2014-a & 1,2 & 1,2 & 3893 \\
\hline 6 & 04.11 .14 & vecl-spANP2-osk-016-03+01 & 04-Nov-2014-a & $1, \mathrm{mu}$ & 1,2 & 4033 \\
\hline 7 & 06.11 .14 & vecl-spANP2-osk-018-03+01 & 06-Nov-2014-d & 1,2 & 1,2 & 4808 \\
\hline 8 & 07.11 .14 & vecl-spANP2-osk-019-01+01 & 07-Nov-2014-a & 1,2 & 1,2 & 4380 \\
\hline 9 & 11.11 .14 & vecl-spANP2-osk-020-02+01 & 11-Nov-2014-c & 10,2 & 2 & 4494 \\
\hline 10 & 12.11 .14 & vecl-spANP2-osk-021-02+01 & 12-Nov-2014-a & 2 & 2 & 4062 \\
\hline 11 & 13.11.14 & vecl-spANP2-osk-022-02+01 & 13-Nov-2014a & $10,2,1$ & 1,2 & 4365 \\
\hline 12 & 25.11 .14 & vecl-spANP2-osk-023-01+01 & 25-Nov-2014a & 1 & 1 & 4429 \\
\hline 13 & 27.11 .14 & vecl-spANP2-osk-025-01+01 & 27-Nov-2014a & 2 & 2 & 4882 \\
\hline 14 & 28.11 .14 & vecl-spANP2-osk-026-01+01 & 28-Nov-2014a & 1,2 & 1,2 & 4533 \\
\hline 15 & 03.12 .14 & vecl-spANP2-osk-028-01+01 & 03-Dec-2014a & 9 & 1 & 5050 \\
\hline 16 & 04.12 .14 & vecl-spANP2-osk-029-01+01 & 04-Dec-2014a & 2 & 1,2 & 4402 \\
\hline 17 & 04.12 .14 & vecl-spANP2-osk-029-02+01 & 04-Dec-2014a & 2 & 1,2 & 4083 \\
\hline 18 & 10.12 .14 & vecl-spANP2-osk-030-01+01 & 10-Dec-2014a & 1 & 1 & 4087 \\
\hline 19 & 11.12 .14 & vecl-spANP2-osk-031-01+01 & 11-Dec-2014b & 1 & 1,2 & 5097 \\
\hline 20 & 12.12 .14 & vecl-spANP2-osk-032-01+01 & 12-Dec-2014a & 1 & 1,2 & 4786 \\
\hline 21 & 18.12 .14 & vecl-spANP2-osk-034-01+01 & 18-Dec-2014 & 1 & 1 & 4678 \\
\hline 22 & 06.01 .15 & vecl-spANP2-osk-035-01+01 & 06-Jan-2015a & 1 & 1 & 7721 \\
\hline 23 & 07.01 .15 & vecl-spANP2-osk-036-01+01 & 07-Jan-2015a & $1, \mathrm{mu}$ & 1,2 & 3998 \\
\hline 24 & 08.01 .15 & vecl-spANP2-osk-037-02+01 & 08-Jan-2015b & $\mathrm{mu}, 2$ & 1,2 & 4671 \\
\hline 25 & 09.01 .15 & vecl-spANP2-osk-038-01+01 & 09-Jan-2015a & 1 & 1,2 & 4145 \\
\hline 26 & 09.01 .15 & vecl-spANP2-osk-038-02+01 & 09-Jan-2015a & 1 & 1 & 3829 \\
\hline 27 & 13.01 .15 & vecl-spANP2-osk-039-01+01 & 13-Jan-2015a & 2 & 2 & 4266 \\
\hline 28 & 15.01 .15 & vecl-spANP2-osk-041-02+01 & 15-Jan-2015b & 1,9 & 1 & 4930 \\
\hline 29 & 16.01 .15 & vecl-spANP2-osk-042-01+01 & 16-Jan-2015a & 1,2 & 1,2 & 4684 \\
\hline 30 & 16.01 .15 & vecl-spANP2-osk-042-02+01 & 16-Jan-2015b & $\mathrm{mu}, 2$ & 1,2 & 4621 \\
\hline 31 & 28.01 .15 & vecl-spANP2-osk-048-01+01 & 28-Jan-2015a & $1, \mathrm{mu}$ & 1 & 5427 \\
\hline 32 & 29.01 .15 & vecl-spANP2-osk-049-01+01 & 29-Jan-2015a & 1,2 & 1,2 & 5361 \\
\hline 33 & 03.02 .15 & vecl-spANP2-osk-050-01+01 & 03-Feb-2015a & 1 & 1 & 6039 \\
\hline 34 & 04.02 .15 & vecl-spANP2-osk-051-01+01 & 04-Feb-2015a & 2 & 2 & 5993 \\
\hline 35 & 05.02 .15 & vecl-spANP2-osk-052-01+01 & 05-Feb-2015a & 2 & 2 & 6155 \\
\hline 36 & 06.02 .15 & vecl-spANP2-osk-053-01+01 & 06-Feb-2015a & 1 & 1 & 7059 \\
\hline 37 & 10.02 .15 & vecl-spANP2-osk-054-02+01 & 10-Feb-2015b & 2 & 2 & 7052 \\
\hline 38 & 11.02 .15 & vecl-spANP2-osk-055-01+01 & $11-F e b-2015 b$ & 1 & 1,2 & 7663 \\
\hline 39 & 24.02 .15 & vecl-spANP2-osk-057-01+01 & 24-Feb-2015a & 2 & 2 & 4891 \\
\hline 40 & 25.02 .15 & vecl-spANP2-osk-058-01+01 & $25-F e b-2015 b$ & Ifp & 1,2 & 7000 \\
\hline 41 & 27.02 .15 & vecl-spANP2-osk-060-02+01 & 27-Feb-2015a & 2 & 1,2 & 7771 \\
\hline 42 & 03.03 .15 & vecl-spANP2-osk-061-01+01 & 03-Mar-2015a & Ifp & 1,2 & 7250 \\
\hline 43 & 04.03 .15 & vecl-spANP2-osk-062-01+01 & 04-Mar-2015a & 2 & 1,2 & 7039 \\
\hline 44 & 04.03 .15 & vecl-spANP2-osk-062-03+01 & 04-Mar-2015a & 1 & 1,2 & 7088 \\
\hline 45 & 05.03 .15 & vecl-spANP2-osk-063-01+01 & 05-Mar-2015a & 1 & 1 & 6429 \\
\hline 46 & 06.03 .15 & vecl-spANP2-osk-064-01+01 & 06-Mar-2015a & Ifp & 1,2 & 7500 \\
\hline 47 & 10.03 .15 & vecl-spANP2-osk-065-01+01 & 10-Mar-2015a & 2 & 2 & 6259 \\
\hline 48 & 25.03 .15 & vecl-spANP2-osk-070-01+01 & 25-Mar-2015a & 1 & 1 & 5800 \\
\hline 49 & 26.03 .15 & vecl-spANP2-osk-071-01+01 & 26-Mar-2015a & 2 & 2 & 7087 \\
\hline 50 & 26.03 .15 & vecl-spANP2-osk-071-02+01 & 26-Mar-2015a & 1 & 1 & 7177 \\
\hline 51 & 27.03 .15 & vecl-spANP2-osk-072-01+01 & 27-Mar-2015a & 1 & 1 & 6812 \\
\hline 52 & 31.03 .15 & vecl-spANP2-osk-073-01+01 & 31-Mar-2015a & 2 & 2 & 7337 \\
\hline 53 & 31.03 .15 & vecl-spANP2-osk-073-01+01 & 31-Mar-2015a & 10 & 2 & 7337 \\
\hline
\end{tabular}


Scopolamine monkey O

\begin{tabular}{|c|c|c|c|c|c|c|c|c|c|}
\hline no. & noDirectionsUsed & \multirow{2}{*}{$\begin{array}{l}\text { prefDir } \\
80\end{array}$} & \multirow{2}{*}{$\begin{array}{r}\text { prefSpeed } \\
12\end{array}$} & \multirow{2}{*}{$\begin{array}{r}\text { xStimulus } \\
-18\end{array}$} & \multicolumn{2}{|c|}{ yStimulus degreeOfResponseEvent } & \multicolumn{2}{|c|}{ substancelnjected } & concentration amountOfCycles \\
\hline 1 & 2 & & & & 6,2 & 35 & sco & 0,05 & 1 \\
\hline 2 & 2 & 330 & 12 & $-6,2$ & 4,8 & 30 & sco & 0,05 & 1 \\
\hline 3 & 2 & 30 & 8 & $-7,9$ & 4,3 & 30 & sco & 0,05 & 2 \\
\hline 4 & 2 & 30 & 12 & $-10,7$ & 4,3 & 30 & sco & 0,05 & 1 \\
\hline 5 & 2 & 30 & 12 & $-9,7$ & 1,7 & 30 & sco & 0,05 & 1 \\
\hline 6 & 2 & 30 & 12 & $-8,6$ & 4,8 & 30 & sco & 0,05 & 1 \\
\hline 7 & 2 & 230 & 8 & -8 & 3,1 & 30 & sco & 0,05 & 1 \\
\hline 8 & 2 & 90 & 12 & $-7,4$ & 3,4 & 30 & sco & 0,05 & 1 \\
\hline 9 & 2 & 90 & 8 & $-7,5$ & 3,4 & 30 & sco & 0,05 & 1 \\
\hline 10 & 2 & 10 & 12 & $-8,7$ & 4,9 & 30 & sco & 0,05 & 1 \\
\hline 11 & 2 & 90 & 12 & $-10,9$ & 3,4 & 30 & sco & 0,05 & 1 \\
\hline 12 & 2 & 150 & 8 & $-9,9$ & 3,4 & 30 & sco & 0,05 & 1 \\
\hline 13 & 2 & 240 & 12 & $-7,4$ & 4,4 & 30 & sco & 0,05 & 2 \\
\hline 14 & 2 & 120 & 12 & $-6,1$ & 3,8 & 30 & sco & 0,05 & 3 \\
\hline 15 & 2 & 210 & 12 & $-6,9$ & 7,9 & 30 & sco & 0,1 & 1 \\
\hline 16 & 2 & 90 & 12 & $-9,5$ & 3,8 & 30 & sco & 0,1 & 1 \\
\hline 17 & 2 & 90 & 12 & $-9,5$ & 3,8 & 30 & sco & 0,1 & 1 \\
\hline 18 & 2 & 120 & 12 & -11 & 6,4 & 30 & sco & 0,1 & 2 \\
\hline 19 & 2 & 135 & 12 & $-7,3$ & 6,3 & 30 & sco & 0,1 & 1 \\
\hline 20 & 2 & 140 & 12 & $-7,8$ & 4 & 30 & sco & 0,1 & 1 \\
\hline 21 & 2 & 90 & 8 & $-10,5$ & 2,9 & 30 & sco & 0,1 & 1 \\
\hline 22 & 2 & 280 & 12 & $-6,3$ & 6,2 & 30 & sco & 0,1 & 1 \\
\hline 23 & 2 & 200 & 12 & -9 & 3,3 & 30 & sco & 0,1 & 1 \\
\hline 24 & 2 & 160 & 4 & $-7,1$ & 5,3 & 30 & sco & 0,1 & 1 \\
\hline 25 & 2 & 240 & 8 & $-8,5$ & 4,3 & 30 & sco & 0,1 & 1 \\
\hline 26 & 2 & 60 & 8 & $-8,5$ & 4,3 & 30 & sco & 0,1 & 1 \\
\hline 27 & 2 & 200 & 8 & $-5,7$ & 5,5 & 30 & sco & 0,1 & 1 \\
\hline 28 & 2 & 240 & 12 & $-6,2$ & 5,9 & 30 & sco & 0,1 & 1 \\
\hline 29 & 2 & 140 & 12 & $-12,7$ & 5,3 & 30 & sco & 0,1 & 1 \\
\hline 30 & 2 & 140 & 12 & $-8,3$ & 4,5 & 30 & sco & 0,1 & 2 \\
\hline 31 & 2 & 300 & 12 & $-8,7$ & 7,8 & 30 & sco & 0,1 & 2 \\
\hline 32 & 2 & 270 & 12 & $-9,3$ & 6,7 & 30 & sco & 0,1 & 2 \\
\hline 33 & 2 & 215 & 12 & $-3,6$ & 5,9 & 30 & sco & 0,1 & 1 \\
\hline 34 & 2 & 255 & 12 & $-6,2$ & 7,2 & 30 & sco & 0,1 & 3 \\
\hline 35 & 2 & 250 & 12 & $-6,4$ & 7,5 & 30 & sco & 0,1 & 2 \\
\hline 36 & 2 & 0 & 8 & $-5,4$ & 7,7 & 30 & sco & 0,1 & 1 \\
\hline 37 & 2 & 300 & 12 & & & 30 & sco & 0,1 & 1 \\
\hline 38 & 2 & 90 & 12 & $-1,5$ & 6,7 & 25 & sco & 0,1 & 2 \\
\hline 39 & 2 & 120 & 12 & $-8,6$ & 4,4 & 30 & sco & 0,1 & 1 \\
\hline 40 & 8 & 0 & 8 & $-2,4$ & 4,9 & 30 & sco & 0,1 & 1 \\
\hline 41 & 2 & 155 & 12 & $-4,5$ & 7,7 & 30 & sco & 0,1 & 1 \\
\hline 42 & 8 & & 12 & -2 & 5 & 30 & sco & 0,1 & 2 \\
\hline 43 & 2 & 0 & 12 & $-3,5$ & 7,5 & 30 & sco & 0,1 & 1 \\
\hline 44 & 2 & 0 & 12 & $-3,5$ & 7,5 & 30 & sco & 0,1 & 1 \\
\hline 45 & 2 & 190 & 8 & $-1,8$ & 7,4 & 30 & sco & 0,1 & 1 \\
\hline 46 & 8 & & 8 & $-1,5$ & 4,6 & 30 & sco & 0,1 & 2 \\
\hline 47 & 2 & & & & & & sco & 0,1 & 1 \\
\hline 48 & 2 & & & & & & sco & 0,1 & 1 \\
\hline 49 & 2 & & & & & & sco & 0,1 & 1 \\
\hline 50 & 2 & & & & & & sco & 0,1 & 1 \\
\hline 51 & 2 & & & & & & sco & 0,1 & 1 \\
\hline 52 & 2 & & & & & & sco & 0,1 & 2 \\
\hline 53 & 2 & & & & & & sco & 0,1 & 2 \\
\hline
\end{tabular}


Scopolamine monkey O

\begin{tabular}{|c|c|c|c|c|c|c|c|c|}
\hline no. $y$ & velocity(nl/s) & ) injectionrate(nl/min) & trialBegin1 & trialEnd1 & trialBegin2 & trialEnd 2 & trialBegin3 & trialEnd 3 \\
\hline 1 & 1 & 2 & 243 & 356 & & & & \\
\hline 2 & 1 & 2 & 162 & 285 & & & & \\
\hline 3 & 1 & 2 & 129 & 250 & 485 & 604 & & \\
\hline 4 & 1 & 2 & 198 & 359 & & & & \\
\hline 5 & 1 & 2 & 157 & 262 & & & & \\
\hline 6 & 1 & 2 & 263 & 436 & & & & \\
\hline 7 & 1 & 2 & 275 & 457 & & & & \\
\hline 8 & 1 & 2 & 124 & 237 & & & & \\
\hline 9 & 1 & 2 & 257 & 430 & & & & \\
\hline 10 & 1 & 2 & 163 & 163 & & & & \\
\hline 11 & 1 & 2 & 239 & 300 & & & & \\
\hline 12 & 1 & 2 & 284 & 508 & & & & \\
\hline 13 & 1 & 2 & 160 & 282 & 614 & 738 & & \\
\hline 14 & 1 & 2 & 131 & 237 & 480 & 599 & 901 & 1021 \\
\hline 15 & 1 & 2 & 200 & 310 & & & & \\
\hline 16 & 1 & 2 & 152 & 269 & & & & \\
\hline 17 & 1 & 2 & 136 & 257 & & & & \\
\hline 18 & 1 & 2 & 239 & 412 & 680 & 797 & & \\
\hline 19 & 1 & 2 & 184 & 298 & & & & \\
\hline 20 & 1 & 2 & 135 & 253 & & & & \\
\hline 21 & 1 & 2 & 149 & 270 & & & & \\
\hline 22 & 1 & 2 & 196 & 319 & & & & \\
\hline 23 & 1 & 2 & 190 & 306 & & & & \\
\hline 24 & 1 & 2 & 274 & 444 & & & & \\
\hline 25 & 1 & 2 & 170 & 290 & & & & \\
\hline 26 & 1 & 2 & 176 & 360 & & & & \\
\hline 27 & 1 & 2 & 178 & 355 & & & & \\
\hline 28 & 1 & 2 & 238 & 411 & & & & \\
\hline 29 & 1 & 2 & 280 & 446 & & & & \\
\hline 30 & 1 & 2 & 126 & 310 & 524 & 644 & & \\
\hline 31 & 1 & 2 & 182 & 360 & 609 & 727 & & \\
\hline 32 & 1 & 2 & 135 & 259 & 591 & 704 & & \\
\hline 33 & 1 & 2 & 313 & 419 & & & & \\
\hline 34 & 2 & 2 & 178 & 349 & 643 & 761 & 994 & 1115 \\
\hline 35 & 2 & 2 & 203 & 364 & 629 & 725 & & \\
\hline 36 & 2 & 2 & 176 & 352 & & & & \\
\hline 37 & 2 & 2 & 196 & 374 & & & & \\
\hline 38 & 2 & 4 & 315 & 437 & 630 & 751 & & \\
\hline 39 & 2 & 4 & 329 & 416 & & & & \\
\hline 40 & 2 & 4 & 352 & 468 & & & & \\
\hline 41 & 2 & 4 & 192 & 370 & & & & \\
\hline 42 & 2 & 4 & 358 & 473 & 707 & 886 & & \\
\hline 43 & 2 & 4 & 160 & 270 & & & & \\
\hline 44 & 2 & 4 & 217 & 339 & & & & \\
\hline 45 & 2 & 4 & 279 & 351 & & & & \\
\hline 46 & 2 & 4 & 372 & 549 & 990 & 1109 & & \\
\hline 47 & 2 & 4 & 181 & 242 & & & & \\
\hline 48 & 2 & 4 & 138 & 312 & & & & \\
\hline 49 & 2 & 4 & 157 & 280 & & & & \\
\hline 50 & 2 & 4 & 145 & 283 & & & & \\
\hline 51 & 2 & 4 & 162 & 339 & & & & \\
\hline 52 & 2 & 4 & 216 & 337 & 633 & 778 & & \\
\hline 53 & 2 & 4 & 216 & 337 & 633 & 778 & & \\
\hline
\end{tabular}




\begin{tabular}{|c|c|c|c|c|c|c|c|}
\hline no. & plexStart1 & plexEnd1 & plexStart2 & plexEnd2 & plexStart3 & plexEnd 3 & exclude cells \\
\hline 1 & $00: 21: 00$ & 00:31:00 & & & & & \\
\hline 2 & $00: 15: 00$ & $00: 25: 00$ & & & & & \\
\hline 3 & $00: 11: 00$ & $00: 21: 00$ & $00: 41: 00$ & $00: 51: 00$ & & & \\
\hline 4 & $00: 17: 00$ & $00: 32: 00$ & & & & & \\
\hline 5 & $00: 16: 00$ & $00: 25: 00$ & & & & & \\
\hline 6 & $00: 23: 00$ & $00: 38: 00$ & & & & & \\
\hline 7 & $00: 24: 00$ & $00: 39: 00$ & & & & & \\
\hline 8 & $00: 11: 00$ & $00: 21: 00$ & & & & & \\
\hline 9 & $00: 22: 00$ & $00: 37: 00$ & & & & & \\
\hline 10 & $00: 14: 00$ & $00: 24: 00$ & & & & & \\
\hline 11 & $00: 22: 00$ & $00: 29: 00$ & & & & & \\
\hline 12 & $00: 26: 00$ & $00: 46: 00$ & & & & & \\
\hline 13 & $00: 13: 00$ & $00: 23: 00$ & $00: 57: 00$ & 01:07:00 & & & \\
\hline 14 & $00: 11: 00$ & $00: 21: 00$ & $0: 41: 00$ & $0: 51: 00$ & 01:20:00 & 01:30:00 & \\
\hline 15 & $00: 20: 00$ & $00: 30: 00$ & & & & & \\
\hline 16 & $00: 13: 00$ & $00: 23: 00$ & & & & & \\
\hline 17 & $00: 12: 00$ & $00: 22: 00$ & & & & & \\
\hline 18 & $00: 21: 00$ & $00: 36: 00$ & 01:00:00 & 01:10:00 & & & \\
\hline 19 & $00: 16: 00$ & $00: 26: 00$ & & & & & \\
\hline 20 & $00: 12: 00$ & $00: 22: 00$ & & & & & \\
\hline 21 & $00: 13: 00$ & $00: 23: 00$ & & & & & \\
\hline 22 & $00: 18: 00$ & $00: 28: 00$ & & & & & \\
\hline 23 & $00: 16: 00$ & $00: 26: 00$ & & & & & \\
\hline 24 & $00: 25: 00$ & $00: 40: 00$ & & & & & \\
\hline 25 & $00: 15: 00$ & $00: 25: 00$ & & & & & \\
\hline 26 & $00: 15: 00$ & 00:30:00 & & & & & \\
\hline 27 & $00: 15: 00$ & $00: 30: 00$ & & & & & \\
\hline 28 & $00: 21: 00$ & $00: 31: 00$ & & & & & 525:567, \\
\hline 29 & $00: 26: 00$ & $00: 41: 00$ & & & & & \\
\hline 30 & $00: 11: 00$ & $00: 26: 00$ & $00: 44: 00$ & $00: 54: 00$ & & & \\
\hline 31 & $00: 16: 00$ & $00: 31: 00$ & $00: 54: 00$ & 01:04:00 & & & \\
\hline 32 & $00: 12: 00$ & $00: 22: 00$ & $00: 50: 00$ & 01:00:00 & & & \\
\hline 33 & $00: 26: 00$ & 00:38:00 & & & & & 393:end \\
\hline 34 & $00: 16: 00$ & $00: 31: 00$ & $00: 57: 00$ & 01:07:00 & 01:27:00 & 01:37:00 & \\
\hline 35 & $00: 20: 00$ & $00: 35: 00$ & 01:00:00 & 01:10:00 & & & \\
\hline 36 & $00: 17: 00$ & $00: 32: 00$ & & & & & 368:end \\
\hline 37 & $00: 18: 00$ & $00: 33: 00$ & & & & & \\
\hline 38 & $00: 27: 30$ & $00: 37: 30$ & 00:58:00 & 01:08:00 & & & \\
\hline 39 & $00: 30: 00$ & $00: 40: 00$ & & & & & \\
\hline 40 & 00:30:00 & $00: 40: 00$ & & & & & \\
\hline 41 & $00: 16: 00$ & $00: 31: 00$ & & & & & \\
\hline 42 & $00: 30: 00$ & $00: 40: 00$ & 01:00:00 & 01:15:00 & & & \\
\hline 43 & $00: 15: 00$ & $00: 25: 00$ & & & & & \\
\hline 44 & $00: 20: 00$ & $00: 30: 00$ & & & & & \\
\hline 45 & $00: 25: 00$ & $00: 35: 00$ & & & & & \\
\hline 46 & $00: 32: 00$ & $00: 47: 00$ & 01:25:00 & 01:35:00 & & & \\
\hline 47 & $00: 16: 00$ & $00: 24: 00$ & & & & & 234:end \\
\hline 48 & $00: 12: 00$ & $00: 27: 00$ & & & & & \\
\hline 49 & $00: 13: 00$ & $00: 23: 00$ & & & & & \\
\hline 50 & $00: 12: 00$ & $00: 27: 00$ & & & & & 340:end \\
\hline 51 & $00: 16: 00$ & $00: 31: 00$ & & & & & $0: 42$ \\
\hline 52 & $00: 22: 00$ & $00: 32: 00$ & $00: 57: 00$ & 01:12:00 & & & \\
\hline 53 & $00: 22: 00$ & $00: 32: 00$ & $00: 57: 00$ & 01:12:00 & & & 680 :end \\
\hline
\end{tabular}




\begin{tabular}{|c|c|c|c|c|c|}
\hline no. & date & filename & eparUsed & mclabUnitNo & electrodeNo \\
\hline 1 & 01.08 .12 & vecl-spANPtc-pie-001-01+01 & 1Aug-a & 2 & 2 \\
\hline 2 & 01.08 .12 & vecl-spANPtc-pie-001-01+02 & 1Aug-b & 10 & 2,3 \\
\hline 3 & 02.08 .12 & vecl-spANPtc-pie-002-01+01 & 2Aug-a & 2 & \\
\hline 4 & 02.08 .12 & vecl-spANPtc-pie-002-01+02 & 2Aug-a & 2 & 2 \\
\hline 5 & 07.08 .12 & vecl-spANPtc-pie-003-01+01 & 16th May & Ifp & 2,3 \\
\hline 6 & 09.08 .12 & vecl-spANPtc-pie-005-01+01 & 09Aug-a & 11 & 3 \\
\hline 7 & 10.08 .12 & vecl-spANPtc-pie-006-01+01 & 10Aug-a & 11 & 3 \\
\hline 8 & 14.08 .12 & vecl-spANPtc-pie-007-01+01 & 16May & Ifp & 2,3 \\
\hline 9 & 16.08.12 & vecl-spANPtc-pie-008-01+01 & 16May & Ifp & 2,3 \\
\hline 10 & 17.08 .12 & vecl-spANPtc-pie-009-01+01 & 17Auga & 11 & \\
\hline 11 & 17.08 .12 & vecl-spANPtc-pie-010-01+02 & 17Auga & Ifp & 2,3 \\
\hline 12 & 23.08 .12 & vecl-spANPtc-pie-012-01+01 & 23Aug-a & 11 & 年 \\
\hline 13 & 28.08.13 & vecl-spANPtc-pie-013-01+01 & 28Aug-b & Ifp & 2,3 \\
\hline 14 & 29.08 .12 & vecl-spANPtc-pie-014-01+01 & 29Auga & 2 & 2,3 \\
\hline 15 & 29.08 .12 & vecl-spANPtc-pie-014-01+03 & 29Aug-b & 2 & $2+2$ \\
\hline 16 & 05.09 .12 & vecl-spANPtc-pie-015-01+01 & 5Sep-a & 1 & 1 \\
\hline 17 & 06.09.12 & vecl-spANPtc-pie-016-01+01 & 6Sept-a & 1 & \\
\hline 18 & 07.09 .12 & vecl-spANPtc-pie-017-01+01 & 07sep-a & 11 & \\
\hline 19 & 11.09 .12 & vecl-spANPtc-pie-018-01+01 & 11Sep-a & 11 & \\
\hline 20 & 12.09 .12 & vecl-spANPtc-pie-019-01+01 & 16May & Ifp & 2,3 \\
\hline 21 & 13.09.12 & vecl-spANPtc-pie-020-01+01 & 13Sep-a & 1 & \\
\hline 22 & 13.09 .12 & vecl-spANPtc-pie-020-01+02 & 13Sep-b & 1 & \\
\hline 23 & 18.09.12 & vecl-spANPtc-pie-021-01+01 & 18Sep-a & 1 & \\
\hline 24 & 19.09 .12 & vecl-spANPtc-pie-022-01+01 & 16May & Ifp & 1,3 \\
\hline 25 & 20.09 .12 & vecl-spANPtc-pie-023-01+01 & 20Sept-a & 1,11 & 1,3 \\
\hline 26 & 20.09 .12 & vecl-spANPtc-pie-023-01+02 & 20Sept-a & 1,11 & 1,3 \\
\hline 27 & 16.10 .12 & vecl-spANPtc-pie-026-01+01 & $160 c t-a$ & 11 & 3 \\
\hline 28 & 18.10.12 & vecl-spANPtc-pie-028-01+01 & 180ct-a & 1 & \\
\hline 29 & 22.11 .12 & vecl-spANPtc-pie-036-01+01 & 250ct-a & Ifp & \\
\hline 30 & 27.11.12 & vecl-spANPtc-pie-037-01+01 & $27 \mathrm{Nov}-\mathrm{a}$ & 11 & \\
\hline 31 & 22.05 .12 & vecl-spANP1-pie-014-01+02 & 22May-a & $\mathrm{mu}$ & 2,3 \\
\hline 32 & 22.05.12 & vecl-spANP1-pie-014-01+03 & 22May-a & 2 & 2,3 \\
\hline 33 & 23.05.12 & vecl-spANP1-pie-015-01+01 & 23May-a & 2 & 2,3 \\
\hline 34 & 26.06 .12 & vecl-spANP1-pie-022-01+01 & 26Jun-a & 11 & 3 \\
\hline 35 & 28.06 .12 & vecl-spANP1-pie-023-01+02 & 28Jun-b & 2 & \\
\hline 36 & 29.06 .12 & vecl-spANP1-pie-024-01+02 & 29Jun-a & 11 & 2,3 \\
\hline 37 & 29.06 .12 & vecl-spANP1-pie-024-01+03 & 29Jun-a & 11 & 2,3 \\
\hline 38 & 03.07 .12 & vecl-spANP1-pie-025-01+01 & 3Jul-a & 2 & 2 \\
\hline 39 & 04.07 .12 & vecl-spANP1-pie-026-01+01 & 4Jul-b & 11 & 3 \\
\hline 40 & 04.07.12 & vecl-spANP1-pie-026-01+02 & 4Jul-b & 11 & \\
\hline 41 & 05.07 .12 & vecl-spANP1-pie-027-01+01 & 5Jul-a & 11 & \\
\hline 42 & 10.07.12 & vecl-spANP1-pie-030-01+01 & 10Jul-b & 2 & \\
\hline 43 & 11.07 .12 & vecl-spANP1-pie-031-01+01 & 11Jul-a & 2 & \\
\hline 44 & 11.07 .12 & vecl-spANP1-pie-031-01+02 & 11Jul-a & 2 & \\
\hline 45 & 12.07 .12 & vecl-spANP1-pie-032-01+01 & 12Jul-a: & ded & \\
\hline 46 & 13.07.12 & vecl-spANP1-pie-033-01+01 & 13Jul-a & 2 & \\
\hline 47 & 19.07.12 & vecl-spANP1-pie-035-01+01 & 19-Jul-a & 2 & \\
\hline 48 & 31.07 .12 & vecl-spANP1-pie-037-01+02 & 31Jul-a & 2 & \\
\hline 49 & 23.07.13 & vecl-spANP2-pie-010-01+01 & 23Jul-a & $\mathrm{mu}$ & 1,2 \\
\hline 50 & 24.07 .13 & vecl-spANP2-pie-011-01+01 & 24Julb & $\mathrm{mu}$ & 1,2 \\
\hline 51 & 25.07 .13 & vecl-spANP2-pie-012-01+01 & 26Jul_a & $\mathrm{mu}$ & 1,2 \\
\hline 52 & 26.07.13 & vecl-spANP2-pie-013-01+01 & 30Jul_a & $\mathrm{mu}$ & 1,2 \\
\hline 53 & 30.07 .13 & vecl-spANP2-pie-014-01+01 & 25Jul_a & $\mathrm{mu}$ & \\
\hline 54 & 01.08 .13 & vecl-spANP2-pie-015-01+02 & 1Aug-a & $\mathrm{mu}$ & \\
\hline 55 & 08.08 .13 & vecl-spANP2-pie-017-02+01 & 8 Aug-b & 2 & \\
\hline 56 & 09.08 .13 & vecl-spANP2-pie-018-01+01 & 09Aug-d & 2 & 1,2 \\
\hline 57 & 09.08 .13 & vecl-spANP2-pie-018-01+02 & 09Aug-d & 2 & \\
\hline 58 & 14.08 .13 & vecl-spANP2-pie-019-01+01 & 14Aug-a & 1 & 1 \\
\hline 59 & 29.08.13 & vecl-spANP2-pie-020-01+02 & 29Aug-a & 1 & \\
\hline 60 & 29.08 .13 & vecl-spANP2-pie-020-01+03 & 29Aug-a & 1 & 1 \\
\hline 61 & 30.08 .13 & vecl-spANP2-pie-021-02+02 & 30Aug-a & 2 & \\
\hline 62 & 04.09 .13 & vecl-spANP2-pie-023-01+03 & 04Sept-a & 1 & \\
\hline 63 & 05.09.13 & vecl-spANP2-pie-024-01+02 & 05sept-a & 2,10 & 2 \\
\hline 64 & 05.09 .13 & vecl-spANP2-pie-024-02+02 & 05Sept-b & 1 & \\
\hline 65 & 25.09.13 & vecl-spANP2-pie-034-01+02 & 25Sept-a & 9 & 1 \\
\hline 66 & 27.09 .13 & vecl-spANP2-pie-035-01+02 & 27Sept-a & 1 & 1 \\
\hline 67 & 27.09 .13 & vecl-spANP2-pie-035-01+03 & 27Sept-a & 1 & 1 \\
\hline 68 & 27.09 .13 & vecl-spANP2-pie-035-01+04 & 27Sept-a & 1 & \\
\hline 69 & 08.10 .13 & vecl-spANP2-pie-039-01+02 & 080ct-a & 1 & \\
\hline 70 & 10.10 .13 & vecl-spANP2-pie-041-02+02 & $100 c t-a$ & exclude trials & 2 \\
\hline 71 & 16.10 .13 & vecl-spANP2-pie-044-01+03 & 160ct-a & exclude trials & \\
\hline 72 & 17.10 .13 & vecl-spANP2-pie-045-01+03 & 170ct-a & $\mathrm{mu}$ & 1,2 \\
\hline 73 & 17.10 .13 & vecl-spANP2-pie-045-02+03 & 170ct-b & $1,2,10$ & 1,2 \\
\hline 74 & 19.11.13 & vecl-spANP2-pie-058-01+02 & 19 Nov-a & $1, \mathrm{mu}$ & 1 \\
\hline 75 & 19.11.13 & vecl-spANP2-pie-058-02+01 & 19 Nov-a & 1 & 1 \\
\hline 76 & 20.11.13 & vecl-spANP2-pie-059-01+02 & $20 \mathrm{Nov}-\mathrm{a}$ & 1,9 & \\
\hline 77 & 25.11.13 & vecl-spANP2-pie-060-01+02 & $25 \mathrm{Nov}-\mathrm{a}$ & $1 \mathrm{mu}$ & \\
\hline 78 & 26.11 .13 & vecl-spANP2-pie-061-02+02 & $26 \mathrm{Nov}-\mathrm{a}$ & 1 & 1 \\
\hline 79 & 21.12 .13 & vecl-spANP2-pie-067-03+01 & 21Dec-b & 2 & \\
\hline 80 & 08.01.1 & & & 2 & \\
\hline 81 & 16.01 .14 & vecl-spANP2-pie-075-01+01 & 16Jan-a & 1 & \\
\hline 82 & 17.01.14 & vecl-spANP2-pie-076-01+01 & 17Jan-a & exclude trials & \\
\hline
\end{tabular}




\begin{tabular}{|c|c|c|c|c|c|c|c|c|}
\hline no. & depth & noDirectionsUsed & prefDir & prefspeed & xStimulus & yStimulus & degreeOfResponseEvent & substancelnjected \\
\hline 1 & 5021 & 2 & 30 & 12 & 2,5 & 5,1 & 30 & sco \\
\hline 2 & 4407 & 2 & 150 & 12 & 2,5 & 5 & 30 & sco \\
\hline 3 & 4200 & 2 & 30 & 8 & 2,2 & 3,8 & 30 & $\mathrm{scc}$ \\
\hline 4 & 4200 & 2 & 30 & 8 & 2,2 & 3,8 & 30 & sco \\
\hline 5 & 5000 & 8 & & & & & 30 & sco \\
\hline 6 & 4000 & 2 & & & & & 30 & sco \\
\hline 7 & 4353 & 2 & 90 & 12 & 3,8 & 4,6 & 30 & $\mathrm{sco}$ \\
\hline 8 & 4500 & 8 & & & & & 30 & sco \\
\hline 9 & 4500 & 8 & & & & & 30 & sco \\
\hline 10 & 4048 & 2 & 270 & 4 & 3 & 2,6 & 30 & sco \\
\hline 11 & 4500 & 8 & & & & & 30 & sco \\
\hline 12 & 4248 & 2 & 120 & 12 & 4,3 & 4,4 & 30 & $\mathrm{sco}$ \\
\hline 13 & 5000 & 2 & 60 & 12 & 4,9 & 4,2 & 30 & sco \\
\hline 14 & 4031 & 2 & 90 & 8 & 3 & 5,7 & 30 & sco \\
\hline 15 & 4043 & 2 & 330 & 12 & 3,3 & 5,5 & 30 & sco \\
\hline 16 & 4989 & 2 & 60 & 8 & 1,2 & 6,6 & 30 & $\mathrm{sco}$ \\
\hline 17 & 4081 & 2 & 180 & 12 & 2,5 & 5 & 30 & sco \\
\hline 18 & 4000 & 2 & 0 & 8 & 4,2 & 3,9 & 30 & sco \\
\hline 19 & 4174 & 2 & 240 & 12 & 3,1 & 4,4 & 30 & sco \\
\hline 20 & 5800 & 8 & & & & & 30 & sco \\
\hline 21 & 4558 & 2 & 300 & 8 & 5 & 4,2 & 30 & $\mathrm{sco}$ \\
\hline 22 & 5038 & 2 & 180 & 12 & 6,1 & 1 & 30 & sco \\
\hline 23 & 4756 & 2 & 270 & 8 & 6 & 5 & 30 & $\mathrm{sco}$ \\
\hline 24 & 6000 & 2 & & & & & 30 & $\mathrm{sco}$ \\
\hline 25 & 4367 & 2 & 90 & 8 & 6,2 & 5,8 & 30 & $\mathrm{sco}$ \\
\hline 26 & 4254 & 2 & 90 & 8 & 6,2 & 5,8 & 30 & $\mathrm{sco}$ \\
\hline 27 & 4644 & 2 & 30 & 12 & 1,9 & 5,8 & 30 & $\mathrm{sco}$ \\
\hline 28 & 4991 & 2 & 90 & 12 & 3,8 & 7 & 30 & $\mathrm{sco}$ \\
\hline 29 & 4600 & 8 & & & & & 25 & sco \\
\hline 30 & 6200 & 2 & & 6 & 5,5 & 5,8 & 25 & $\mathrm{sco}$ \\
\hline 31 & 2683 & 8 & & 8 & 6,5 & 6,5 & 30 & sco \\
\hline 32 & 2683 & 8 & & 8 & 6,5 & 6,5 & 30 & $\mathrm{sco}$ \\
\hline 33 & 3472 & 8 & & 6 & 2,2 & 5,5 & 30 & sco \\
\hline 34 & 3384 & 2 & 330 & 8 & 1,9 & 2,4 & 30 & $\mathrm{sco}$ \\
\hline 35 & 3633 & 2 & & 8 & 4,2 & 3,2 & 30 & sco \\
\hline 36 & & 2 & & 8 & $\begin{array}{l}4,2 \\
3,5\end{array}$ & $\begin{array}{l}4,2 \\
4,6\end{array}$ & 30 & $\mathrm{sco}$ \\
\hline 37 & & 2 & & 8 & 3,5 & 4,6 & 30 & sco \\
\hline 38 & 3531 & 2 & 60 & 8 & 2,5 & 5 & 30 & $\mathrm{sco}$ \\
\hline 39 & 3405 & 2 & 330 & 12 & 1,9 & 5,5 & 30 & sco \\
\hline 40 & 3385 & 2 & 120 & 12 & 1,9 & 5,5 & 30 & $\mathrm{sco}$ \\
\hline 41 & 3055 & 2 & 270 & 12 & 1,1 & 4,1 & 30 & $\mathrm{sco}$ \\
\hline 42 & 3575 & 2 & 270 & 12 & 2,4 & 5,3 & 30 & $\mathrm{sco}$ \\
\hline 43 & 3754 & 2 & 330 & 12 & 3,3 & 4,3 & 30 & sco \\
\hline 44 & 3540 & 2 & 330 & 12 & 3,3 & 4,3 & 30 & $\mathrm{sco}$ \\
\hline 45 & 3054 & 2 & 210 & 12 & 2,3 & 4,9 & 30 & $\mathrm{sco}$ \\
\hline 46 & 4141 & 2 & 0 & 12 & 1,8 & 4,4 & 30 & $\mathrm{sco}$ \\
\hline 47 & 3675 & 2 & 180 & 12 & 4,1 & 5,2 & 30 & $\mathrm{sco}$ \\
\hline 48 & 4874 & 2 & 180 & 12 & 1,7 & 5,4 & 30 & $\mathrm{sco}$ \\
\hline 49 & 5813 & 8 & & 8 & $-5,7$ & $-2,2$ & 25 & $\mathrm{sco}$ \\
\hline 50 & 5658 & 8 & & 8 & $-5,5$ & $-5,2$ & 25 & $\mathrm{sco}$ \\
\hline 51 & 6375 & 8 & & & $-4,4$ & $\begin{array}{l}-3,5 \\
-1,5\end{array}$ & 25 & $\mathrm{sco}$ \\
\hline 52 & 7050 & 8 & & & $-4,7$ & 5,2 & 25 & sco \\
\hline 53 & 6500 & 8 & & & & & 25 & $\mathrm{sco}$ \\
\hline 54 & & 8 & & & & & 25 & $\mathrm{sco}$ \\
\hline 55 & 7371 & 2 & 240 & 12 & $-0,1$ & $-5,4$ & 25 & $\mathrm{sco}$ \\
\hline 56 & 7245 & 2 & 120 & 8 & $-1,2$ & $-4,6$ & 25 & sco \\
\hline 57 & 7104 & 2 & 120 & 8 & $-1,2$ & $-4,6$ & 25 & $\mathrm{sco}$ \\
\hline 58 & 8353 & 2 & 120 & 4 & $-2,1$ & $-4,5$ & 25 & sco \\
\hline 59 & 7000 & 2 & 60 & 12 & $-2,3$ & $-3,9$ & 25 & $\mathrm{sco}$ \\
\hline 60 & 6893 & 2 & 60 & 12 & $-2,3$ & $-3,9$ & 25 & $\mathrm{sco}$ \\
\hline 61 & 8257 & 2 & 20 & 8 & $-1,4$ & $-5,3$ & 25 & $\mathrm{sco}$ \\
\hline 62 & 6885 & 2 & 0 & 12 & $-4,2$ & $-4,3$ & 25 & sco \\
\hline 63 & 7346 & 2 & 90 & 8 & $-1,7$ & $-6,8$ & 25 & $\mathrm{sco}$ \\
\hline 64 & 7065 & 2 & 0 & 12 & $-2,9$ & -6 & 25 & sco \\
\hline 65 & 7455 & 2 & 200 & 12 & $-3,8$ & $-8,3$ & 35 & $\mathrm{sco}$ \\
\hline 66 & 8633 & 2 & 0 & 12 & $-7,6$ & $-6,6$ & 30 & sco \\
\hline 67 & 8633 & 2 & 0 & 12 & $-7,6$ & $-6,6$ & 30 & $\mathrm{sco}$ \\
\hline 68 & 8442 & 2 & 0 & 12 & $-7,6$ & $-6,6$ & 30 & $\mathrm{sco}$ \\
\hline 69 & 7499 & 2 & 185 & 12 & $-9,1$ & -3 & 30 & $\mathrm{sco}$ \\
\hline 70 & 7656 & 2 & 75 & 12 & $-3,8$ & $-7,3$ & 25 & $\mathrm{sco}$ \\
\hline 71 & 7411 & 2 & 0 & 12 & -2 & $-6,6$ & 25 & $\mathrm{sco}$ \\
\hline 72 & 7785 & 2 & 60 & 12 & $-0,9$ & $-5,7$ & 25 & $\mathrm{sco}$ \\
\hline 73 & 7200 & 2 & 50 & 12 & $-3,5$ & $-8,5$ & 25 & sco \\
\hline 74 & 5419 & 2 & 300 & 12 & $-1,1$ & $-7,1$ & 25 & $\mathrm{sco}$ \\
\hline 75 & 5494 & 2 & 300 & 12 & $-1,1$ & $-7,1$ & 25 & $\mathrm{sco}$ \\
\hline 76 & 5765 & 2 & 130 & 2 & $-4,2$ & $\begin{array}{l}-6,9 \\
-1,9\end{array}$ & 35 & $\mathrm{sco}$ \\
\hline 77 & 6494 & 2 & 105 & 12 & $-2,3$ & $-7,7$ & 25 & sco \\
\hline 78 & 6196 & 2 & 250 & 12 & $-2,3$ & $-6,3$ & 25 & $\mathrm{sco}$ \\
\hline 79 & 6208 & 2 & 210 & 12 & $-7,1$ & 1,7 & 25 & sco \\
\hline 80 & 4898 & 2 & 160 & 4 & $-7,1$ & $-4,5$ & 30 & $\mathrm{sco}$ \\
\hline 81 & 3400 & 2 & 130 & 12 & $-3,5$ & $-5,2$ & 25 & $\mathrm{sco}$ \\
\hline 82 & 3261 & 2 & 80 & 8 & $-8,2$ & $\begin{array}{l}-4,2 \\
-4,3\end{array}$ & 25 & $\mathrm{sco}$ \\
\hline
\end{tabular}




\begin{tabular}{|c|c|c|c|c|c|c|}
\hline no. & plexStart1 & plexEnd1 & plexStart2 & plexEnd2 & plexStart3 & plexEnd 3 \\
\hline 1 & $00: 15: 00$ & $00: 25: 00$ & & & & \\
\hline 2 & $00: 16: 00$ & $00: 26: 00$ & $00: 46: 00$ & $00: 56: 00$ & & \\
\hline 3 & $00: 14: 00$ & $00: 24: 00$ & & & & \\
\hline 4 & 00:15:00 & $00: 28: 00$ & & & & \\
\hline 5 & $00: 30: 00$ & 00:41:00 & & & & \\
\hline 6 & 00:21:00 & $00: 32: 00$ & & & & \\
\hline 7 & 00:12:00 & 00:23:00 & & & & \\
\hline 8 & 00:23:30 & 00:33:30 & & & & \\
\hline 9 & 00:31:00 & 00:41:00 & & & & \\
\hline 10 & 00:16:00 & $00: 26: 00$ & & & & \\
\hline 11 & 00:05:30 & 00:15:30 & & & & \\
\hline 12 & $00: 10: 00$ & $00: 20: 00$ & 00:45:00 & 00:55:00 & & \\
\hline 13 & $00: 22: 00$ & 00:32:00 & $00: 58: 00$ & 01:08:00 & & \\
\hline 14 & $00: 14: 00$ & $00: 24: 00$ & & & & \\
\hline 15 & $00: 23: 00$ & 00:33:00 & 00:51:00 & 01:01:00 & & \\
\hline 16 & $00: 24: 00$ & 00:34:00 & & & & \\
\hline 17 & $00: 13: 00$ & $00: 23: 00$ & $00: 45: 30$ & $00: 55: 30$ & & \\
\hline 18 & 00:21:00 & $00: 31: 00$ & & & & \\
\hline 19 & $00: 17: 00$ & $00: 27: 00$ & 00:55:00 & 01:05:00 & 01:25:00 & 01:35:00 \\
\hline 20 & $00: 25: 00$ & $00: 35: 00$ & $00: 56: 00$ & 01:06:00 & & \\
\hline 21 & 00:13:00 & 00:23:00 & & & & \\
\hline 22 & 00:11:00 & 00:21:00 & & & & \\
\hline 23 & $00: 13: 00$ & 00:23:00 & & & & \\
\hline 24 & $00: 24: 30$ & 00:34:30 & 01:04:30 & 01:14:30 & & \\
\hline 25 & $00: 16: 00$ & $00: 26: 00$ & $00: 55: 30$ & 01:05:30 & & \\
\hline 26 & $00: 24: 00$ & 00:34:00 & & & & \\
\hline 27 & 00:21:00 & $00: 31: 00$ & & & & \\
\hline 28 & $00: 16: 00$ & 00:26:00 & & & & \\
\hline 29 & $00: 25: 00$ & 00:35:00 & & & & \\
\hline 30 & $00: 26: 30$ & 00:36:30 & & & & \\
\hline 31 & $00: 27: 00$ & $00: 37: 00$ & & & & \\
\hline 32 & $00: 17: 30$ & $00: 37: 30$ & & & & \\
\hline 33 & 01:00:00 & 01:21:00 & 01:58:00 & 02:19:00 & 02:47:00 & 02:57:00 \\
\hline 34 & 00:21:00 & 00:36:00 & $00: 51: 00$ & 01:04:00 & 01:16:00 & 01:31:00 \\
\hline 35 & $00: 22: 30$ & $00: 37: 30$ & 00:51:00 & 01:06:00 & & \\
\hline 36 & $00: 18: 30$ & $00: 35: 30$ & & & & \\
\hline 37 & 00:18:00 & $00: 31: 00$ & $00: 44: 30$ & $00: 57: 30$ & & \\
\hline 38 & $00: 27: 00$ & 00:35:00 & & & & \\
\hline $\begin{array}{l}50 \\
39\end{array}$ & $00: 14: 00$ & 00:29:00 & 00:51:30 & 01:07:30 & & \\
\hline 40 & $00: 03: 00$ & $00: 17: 00$ & & & & \\
\hline 41 & $00: 19: 30$ & $00: 37: 30$ & & & & \\
\hline 42 & $00: 16: 00$ & $00: 29: 00$ & & & & \\
\hline 43 & $00: 24: 00$ & $00: 42: 00$ & & & & \\
\hline 44 & $00: 20: 00$ & $00: 36: 00$ & & & & \\
\hline 45 & $00: 25: 00$ & $00: 40: 00$ & 01:08:00 & 01:22:00 & & \\
\hline 46 & $00: 36: 00$ & $00: 48: 00$ & 01:12:00 & 01:26:00 & 01:42:00 & 01:55:00 \\
\hline 47 & $00: 18: 00$ & $00: 27: 00$ & $00: 39: 00$ & $00: 51: 00$ & 01:19:00 & 01:39:00 \\
\hline 48 & 00:16:30 & $00: 26: 30$ & & & & \\
\hline 49 & $00: 28: 00$ & $00: 48: 00$ & 01:06:00 & 01:18:00 & & \\
\hline 50 & $00: 35: 00$ & 00:55:00 & 01:28:00 & 01:38:00 & & \\
\hline 51 & 00:33:00 & 00:48:00 & & & & \\
\hline 52 & 00:42:00 & 01:02:00 & & & & \\
\hline 53 & 01:04:00 & 01:15:00 & & & & \\
\hline 54 & 00:12:00 & $00: 27: 00$ & & & & \\
\hline 55 & $00: 11: 00$ & $00: 21: 00$ & $00: 42: 00$ & $00: 52: 00$ & & \\
\hline 56 & 00:11:00 & $00: 21: 00$ & & & & \\
\hline 57 & $00: 10: 00$ & $00: 20: 00$ & & & & \\
\hline 58 & $00: 10: 00$ & $00: 20: 00$ & 00:40:00 & $00: 50: 00$ & & \\
\hline 59 & $00: 10: 00$ & $00: 20: 00$ & & & & \\
\hline 60 & $00: 08: 00$ & $00: 18: 00$ & & & & \\
\hline 61 & $00: 10: 00$ & $00: 20: 00$ & & & & \\
\hline $\begin{array}{l}61 \\
62\end{array}$ & $00: 15: 00$ & $00: 25: 00$ & & & & \\
\hline 63 & $00: 13: 00$ & $00: 23: 00$ & & & & \\
\hline 64 & $00: 21: 00$ & $00: 31: 00$ & & & & \\
\hline 65 & $00: 17: 00$ & $00: 27: 00$ & & & & \\
\hline 66 & $00: 17: 00$ & $00: 27: 00$ & & & & \\
\hline 67 & $00: 18: 00$ & $00: 28: 00$ & & & & \\
\hline 68 & $00: 16: 00$ & $00: 26: 00$ & & & & \\
\hline 69 & $00: 16: 00$ & $00: 26: 00$ & & & & \\
\hline 70 & $00: 11: 00$ & $00: 21: 00$ & & & & \\
\hline 71 & 00:10:00 & $00: 20: 00$ & 00:40:00 & 00:50:00 & & \\
\hline 72 & $00: 11: 00$ & $00: 21: 00$ & & & & \\
\hline 73 & 00:12:00 & $00: 22: 00$ & & & & \\
\hline 74 & $00: 15: 00$ & $00: 25: 00$ & & & & \\
\hline 75 & 00:21:00 & 00:31:00 & & & & \\
\hline 76 & $00: 12: 00$ & $00: 22: 00$ & $00: 37: 00$ & $00: 47: 00$ & & \\
\hline 77 & $00: 10: 00$ & $00: 20: 00$ & & & & \\
\hline 78 & $00: 11: 00$ & $00: 21: 00$ & & & & \\
\hline 79 & $00: 20: 00$ & $00: 35: 00$ & & & & \\
\hline 80 & $00: 11: 00$ & $00: 21: 00$ & & & & \\
\hline 81 & $00: 10: 00$ & $00: 20: 00$ & & & & \\
\hline 82 & $00: 15: 00$ & $00: 30: 00$ & & & & \\
\hline
\end{tabular}


Mecamylamine monkey $\mathrm{P}$

$\begin{array}{rlcrrr}\text { no. } & \text { date } & \text { filename } & \text { eparUsed } & \text { mclabUnitNo } & \text { electrodeNo } \\ 1 & 25.02 .14 & \text { vecl-spANP2-pie-093-01+02 } & \text { 25Feb-a } & 1 & 1 \\ 2 & 26.02 .14 & \text { vecl-spANP2-pie-094-01+02 } & \text { 26Feb-a } & 2 & 2 \\ 3 & 27.02 .14 & \text { vecl-spANP2-pie-095-02+02 } & \text { 27Feb-a } & 1,2 & 1,2 \\ 4 & 10.03 .14 & \text { vecl-spANP2-pie-098-01+04 } & 10 \text { Mar-a } & 1,2,9,10 & 1,2 \\ 5 & 17.03 .14 & \text { vecl-spANP2-pie-102-01+01 } & 17 \mathrm{Mar} & 1 & 1 \\ 6 & 18.03 .14 & \text { vecl-spANP2-pie-103-01+04 } & 18 \text { Mar-a } & 1,2 & 1,2 \\ 7 & 18.03 .14 & \text { vecl-spANP2-pie-103-02+02 } & 18 \text { Mar-b } & 1,2 & 1,2 \\ 8 & 20.03 .14 & \text { vecl-spANP2-pie-104-03+02 } & \text { 20Mar-c } & 1 & 1\end{array}$


Mecamylamine monkey $\mathrm{P}$

$\begin{array}{rrrrrrrrr}\text { no. } & \text { depth } & \text { noDirectionsUsed } & \text { prefDir } & \text { prefSpeed } & \text { xStimulus } & \text { yStimulus } & \text { degreeOfResponseEvent substancelnjected } \\ 1 & 5019 & 2 & 165 & 4 & -8,1 & 5,5 & 30 & \text { MM } \\ 2 & 6045 & 2 & 310 & 12 & -6,9 & -4,7 & 25 & \text { MM } \\ 3 & 6296 & 2 & 280 & 12 & -2,6 & -4,2 & 25 & \text { MM } \\ 4 & 8503 & 2 & 280 & 12 & -2,6 & -4,2 & 25 & \text { MM } \\ 5 & 6980 & 2 & 300 & 8 & -5,1 & -0,5 & 25 & \text { MM } \\ 6 & 6760 & 2 & 40 & 8 & -2,5 & -3,4 & 25 & \text { MM } \\ 7 & 6276 & 2 & 200 & 8 & -3,3 & -4,5 & 25 & \text { MM } \\ 8 & 4297 & 2 & 40 & 8 & -2,5 & -3,5 & 24 & \text { MM }\end{array}$


Mecamylamine monkey $\mathrm{P}$

$\begin{array}{rrrrrrrr}\text { no. } & \text { concentration } & \text { amountOfCycles } & \text { injectionrate(nl/min) } & \text { trialBegin1 } & \text { trialEnd1 } & \text { plexStart1 } & \text { plexEnd1 } \\ 1 & 0,1 & 1 & 6 & 168 & 291 & 00: 14: 00 & 00: 24: 00 \\ 2 & 0,1 & 1 & 6 & 161 & 291 & 00: 13: 00 & 00: 23: 00 \\ 3 & 0,1 & 1 & 6 & 151 & 277 & 00: 12: 00 & 00: 22: 00 \\ 4 & 0,1 & 1 & 6 & 233 & 340 & 00: 21: 00 & 00: 31: 00 \\ 5 & 0,1 & 1 & 6 & 161 & 287 & 00: 13: 00 & 00: 23: 00 \\ 6 & 0,1 & 1 & 6 & 151 & 280 & 00: 12: 00 & 00: 22: 00 \\ 7 & 0,1 & 1 & 6 & 146 & 269 & 00: 12: 00 & 00: 22: 00 \\ 8 & 0,1 & 1 & 6 & 160 & 280 & 00: 12: 30 & 00: 22: 30\end{array}$




\begin{tabular}{|c|c|c|c|c|c|}
\hline no. & date & filename & eparUsed & mclabUnitNo & electrodeNo \\
\hline 1 & 08.10 .14 & vecl-spANP2-osk-006-02+01 & 08-Oct-2014-c & 1,9 & 1 \\
\hline 2 & 09.10 .14 & vecl-spANP2-osk-007-01+02 & 09-Oct-2014a & 2 & 1,2 \\
\hline 3 & 09.10 .14 & vecl-spANP2-osk-007-02+01 & 09-Oct-2014b & 2 & 2 \\
\hline 4 & 14.10 .14 & vecl-spANP2-osk-008-01+01 & 14-Oct-2014-a & 2 & 2 \\
\hline 5 & 24.10 .14 & vecl-spANP2-osk-011-02+01 & 24-Oct-2014-a & 2 & 2 \\
\hline 6 & 17.12.14 & vecl-spANP2-osk-033-01+01 & 17-Dec-2014b & 2 & 2 \\
\hline 7 & 21.01 .15 & vecl-spANP2-osk-044-01+01 & 21-Jan-2015a & 2,1 & 1,2 \\
\hline 8 & 21.01 .15 & vecl-spANP2-osk-044-02+01 & 21-Jan-2015a & $2, \mathrm{mu}$ & 2 \\
\hline 9 & 21.01 .15 & vecl-spANP2-osk-044-03+01 & 21-Jan-2015b & 2 & 2 \\
\hline 10 & 22.01 .15 & vecl-spANP2-osk-045-01+02 & 22-Jan-2015a & $1, \mathrm{mu}$ & 1,2 \\
\hline 11 & 23.01 .15 & vecl-spANP2-osk-046-01+01 & 23-Jan-2015a & $2, \mathrm{mu}$ & 1,2 \\
\hline 12 & 11.03 .15 & vecl-spANP2-osk-066-01+01 & 11-Mar-2015a & 9 & 1 \\
\hline 13 & 11.03 .15 & vecl-spANP2-osk-066-02+01 & 11-Mar-2015a & 1 & 1 \\
\hline 14 & 11.03 .15 & vecl-spANP2-osk-066-03+01 & 11-Mar-2015a & 2,10 & 2 \\
\hline 15 & 12.03 .15 & vecl-spANP2-osk-067-01+01 & 12-Mar-2015a & 1 & 1 \\
\hline 16 & 12.03 .15 & vecl-spANP2-osk-067-02+01 & 12-Mar-2015a & 1 & 1 \\
\hline 17 & 13.03 .15 & vecl-spANP2-osk-068-01+01 & 13-Mar-2015a & $\mathrm{mu}$ & 1 \\
\hline 18 & 24.03 .15 & vecl-spANP2-osk-069-01+01 & 24-Mar-2015a & 1 & 1 \\
\hline
\end{tabular}

Saline monkey $\mathrm{P}$

\begin{tabular}{|c|c|c|c|c|c|}
\hline no. & date & filename & eparUsed & mclabUnitNo & electrodeNo \\
\hline 1 & 30.05 .13 & vecl-spANP2-pie-001-01+01 & 30May-a & $1,2,10$ & 1,2 \\
\hline 2 & 17.07 .13 & vecl-spANP2-pie-007-01+01 & 17Jula & 2 & 2 \\
\hline 3 & 18.07 .13 & vecl-spANP2-pie-008-01+01 & 18Jula & 1,2 & 1,2 \\
\hline 4 & 19.07.13 & vecl-spANP2-pie-009-01+01 & 19Jula & $2, \mathrm{mu}$ & 1,2 \\
\hline 5 & 13.09 .13 & vecl-spANP2-pie-027-01+02 & 13Sept-a & 2 & 2 \\
\hline 6 & 18.09.13 & vecl-spANP2-pie-030-01+02 & 18Sept-a & $\mathrm{mu}$ & 1,2 \\
\hline 7 & 19.09.13 & vecl-spANP2-pie-031-02+01 & 19Sept-a & $\mathrm{mu}$ & 1,2 \\
\hline 8 & 20.09.13 & vecl-spANP2-pie-032-02+02 & 20Sept-b & 2 & 2 \\
\hline 9 & 04.10 .13 & vecl-spANP2-pie-037-01+01 & 04Nov-b & 1 & \\
\hline 10 & 07.10 .13 & vecl-spANP2-pie-038-01+02 & 70ct-a & $\mathrm{mu}$ & 1,2 \\
\hline 11 & 21.10 .13 & vecl-spANP2-pie-046-01+03 & 210ct-a & 2 & 2 \\
\hline 12 & 23.10 .13 & vecl-spANP2-pie-048-01+04 & 230ct-a & $1,2, \mathrm{mu}$ & 1,2 \\
\hline 13 & 04.11 .13 & vecl-spANP2-pie-052-01+02 & 4Nov-a & 1,9 & 1 \\
\hline 14 & 06.11 .13 & vecl-spANP2-pie-054-01+02 & 6 Nov-a & 2 & 2 \\
\hline 15 & 07.11 .13 & vecl-spANP2-pie-055-01+02 & 7Nov-a & 1,9 & 1 \\
\hline 16 & 07.11 .13 & vecl-spANP2-pie-055-02+02 & 7 Nov-b & 1,9 & 1 \\
\hline 17 & 29.11.13 & vecl-spANP2-pie-063-03+01 & 29Nov-a & 1 & 1 \\
\hline 18 & 02.12 .13 & vecl-spANP2-pie-064-02+01 & 2Dec-a & 2 & 2 \\
\hline 19 & 20.12 .13 & vecl-spANP2-pie-066-02+01 & 20Dec-a & 1 & 1 \\
\hline 20 & 24.01 .14 & vecl-spANP2-pie-080-01+02 & 24Jan-a & 1 & 1 \\
\hline 21 & 10.03 .14 & vecl-spANP2-pie-099-02+01 & 10-Mar-a & $1, \mathrm{mu}$ & 1,2 \\
\hline
\end{tabular}


Saline monkey O

\begin{tabular}{rrrrrrrrr} 
no. & \multicolumn{1}{c}{ depth } & noDirectionsUsed & prefDir & prefSpeed & xStimulus & yStimulus & degreeOfResponseEvent substancelnjected \\
1 & 3080 & 2 & 240 & 12 & $-9,7$ & $-0,7$ & 30 & $\mathrm{NaCl}$ \\
2 & 3000 & 2 & 30 & 12 & $-8,3$ & 3,50 & 30 & $\mathrm{NaCl}$ \\
3 & 2768 & 2 & & & & & & $\mathrm{NaCl}$ \\
4 & 5184 & 2 & 330 & 8 & $-8,3$ & 3,5 & 30 & $\mathrm{NaCl}$ \\
5 & 4614 & 2 & 60 & 12 & $-9,5$ & 5 & 30 & $\mathrm{NaCl}$ \\
6 & 5124 & 2 & 0 & 12 & $-10,5$ & 4,5 & 30 & $\mathrm{NaCl}$ \\
7 & 5000 & 2 & 180 & 12 & $-9,3$ & 4,8 & 30 & $\mathrm{NaCl}$ \\
8 & 4857 & 2 & 180 & 12 & $-9,3$ & 4,8 & 30 & $\mathrm{NaCl}$ \\
9 & 4724 & 2 & 120 & 8 & $-9,3$ & 4,8 & 30 & $\mathrm{NaCl}$ \\
10 & 5469 & 2 & 240 & 8 & $-7,5$ & 6,7 & 30 & $\mathrm{NaCl}$ \\
11 & 5679 & 2 & 100 & 12 & $-7,7$ & 5,7 & 30 & $\mathrm{NaCl}$ \\
12 & 6794 & 2 & & & & & & $\mathrm{NaCl}$ \\
13 & 6644 & 2 & & & & & & $\mathrm{NaCl}$ \\
14 & 6605 & 2 & & & & & & $\mathrm{NaCl}$ \\
15 & 7321 & 2 & & & & & & $\mathrm{NaCl}$ \\
16 & 6889 & 2 & & & & & & $\mathrm{NaCl}$ \\
17 & 5379 & 2 & & & & & & $\mathrm{NaCl}$ \\
18 & 5521 & 2 & & & & & &
\end{tabular}

Saline monkey $\mathrm{P}$

$\begin{array}{rrrrrrrrr}\text { no. } & \text { depth } & \text { noDirectionsUsed } & \text { prefDir } & \text { prefSpeed } & \text { xStimulus } & \text { yStimulus } & \text { degreeOfResponseEvent substancelnjected } \\ 1 & 5000 & 8 & 300 & 12 & -3 & 6,8 & 25 & \mathrm{NaCl} \\ 2 & 7000 & 8 & & 8 & -7 & 2,2 & 25 & \mathrm{NaCl} \\ 3 & 5600 & 2 & & & & & & \mathrm{NaCl} \\ 4 & 5300 & 2 & & & & & & \mathrm{NaCl} \\ 5 & 6262 & 2 & 60 & 12 & -5,6 & -2,7 & 25 & \mathrm{NaCl} \\ 6 & 5110 & 8 & 0 & 8 & -6,1 & -1,8 & 25 & \mathrm{NaCl} \\ 7 & 5257 & 2 & 235 & 12 & -6,1 & -1 & 25 & \mathrm{NaCl} \\ 8 & 7250 & 2 & 145 & 8 & -3,7 & -8,3 & 35 & \mathrm{NaCl} \\ 9 & 6904 & 2 & & & & & & \mathrm{NaCl} \\ 10 & 7496 & 8 & & 8 & -3,3 & -7,5 & 30 & \mathrm{NaCl} \\ 11 & 7218 & 2 & 250 & 12 & -0,5 & -6 & 25 & \mathrm{NaCl} \\ 12 & 8247 & 2 & 125 & 8 & -2,2 & -10,3 & 40 & \mathrm{NaCl} \\ 13 & 6029 & 2 & 150 & 12 & -1,9 & -7,8 & 25 & \mathrm{NaCl} \\ 14 & 7209 & 2 & 95 & 8 & -3,2 & -6,6 & 25 & \mathrm{NaCl} \\ 15 & 7238 & 2 & 80 & 8 & -2,3 & -8,1 & 25 & \mathrm{NaCl} \\ 16 & 7238 & 2 & 120 & 8 & -2,3 & -8,1 & 25 & \mathrm{NaCl} \\ 17 & 6321 & 2 & 110 & 8 & -1,7 & -7,7 & 25 & \mathrm{NaCl} \\ 18 & 5576 & 2 & 110 & 4 & -1,5 & -5,3 & 25 & \mathrm{NaCl} \\ 19 & 6830 & 2 & 120 & 8 & -10,8 & 2,6 & 25 & \mathrm{NaCl} \\ 20 & 5838 & 2 & 60 & 12 & -5,1 & -6,2 & 25 & \mathrm{NaCl} \\ 21 & 8282 & 2 & & & & & & \mathrm{NaCl}\end{array}$


Saline monkey O

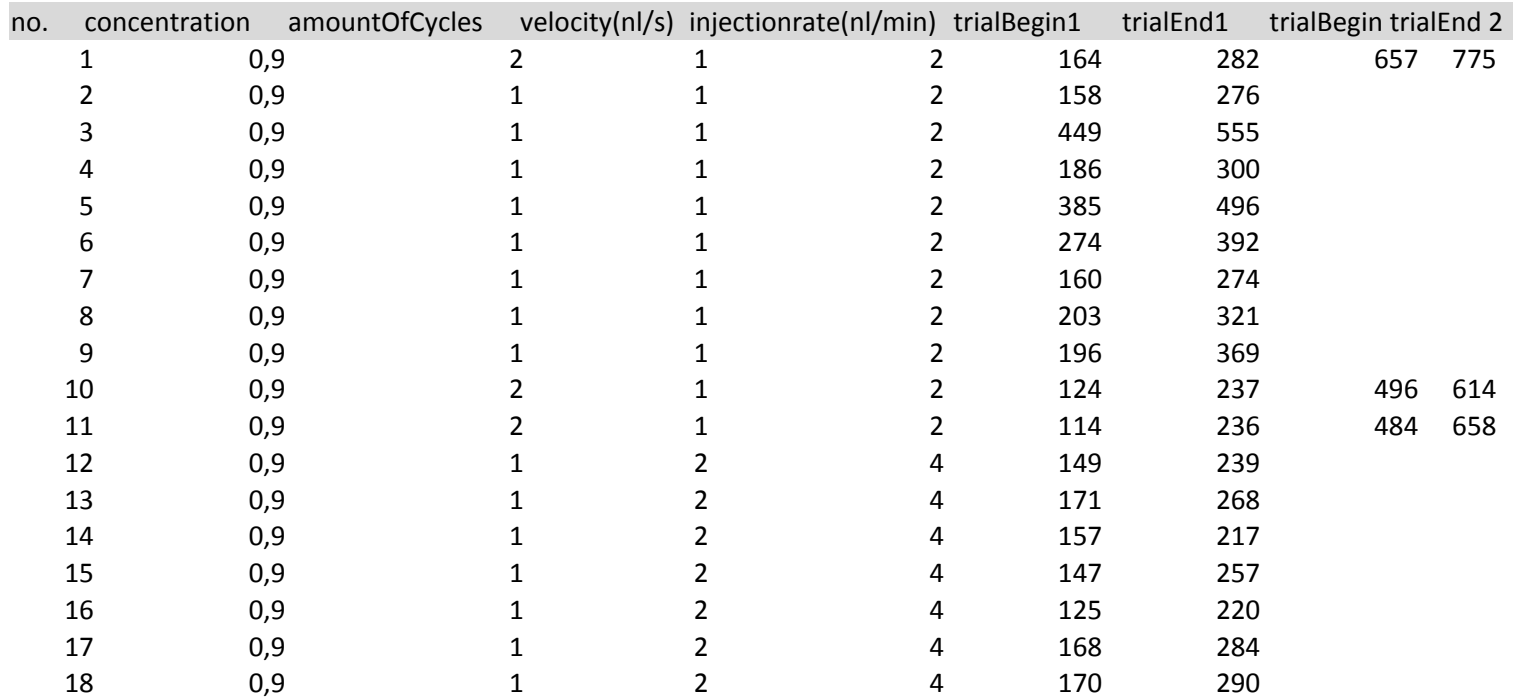

Saline monkey $\mathrm{P}$

\begin{tabular}{|c|c|c|c|c|c|c|c|c|}
\hline no. & concentration & amountOfCycles & velocity $(\mathrm{nl} / \mathrm{s})$ & injectionrate(nl/min) & trialBegin1 & trialEnd1 & trialBegin $t$ & ialEnd 2 \\
\hline 1 & 0,9 & 1 & 2 & 2 & 618 & 725 & & \\
\hline 2 & 0,9 & 1 & 2 & 2 & 294 & 456 & & \\
\hline 3 & 0,9 & 1 & 2 & 2 & 431 & 563 & & \\
\hline 4 & 0,9 & 1 & 2 & 2 & 365 & 500 & & \\
\hline 5 & 0,9 & 2 & 2 & 2 & 142 & 273 & 400 & 499 \\
\hline 6 & 0,9 & 2 & 2 & 2 & 305 & 415 & 665 & 759 \\
\hline 7 & 0,9 & 1 & 2 & 2 & 171 & 296 & & \\
\hline 8 & 0,9 & 2 & 2 & 2 & 145 & 266 & 511 & 617 \\
\hline 9 & 0,9 & 1 & 2 & 2 & 150 & 318 & & \\
\hline 10 & 0,9 & 1 & 2 & 2 & 378 & 499 & & \\
\hline 11 & 0,9 & 1 & 2 & 2 & 173 & 282 & & \\
\hline 12 & 0,9 & 1 & 2 & 2 & 186 & 304 & & \\
\hline 13 & 0,9 & 1 & 2 & 2 & 300 & 412 & & \\
\hline 14 & 0,9 & 1 & 2 & 2 & 112 & 243 & & \\
\hline 15 & 0,9 & 1 & 2 & 2 & 132 & 256 & & \\
\hline 16 & 0,9 & 1 & 2 & 2 & 160 & 284 & & \\
\hline 17 & 0,9 & 1 & 2 & 2 & 120 & 247 & & \\
\hline 18 & 0,9 & 1 & 2 & 2 & 180 & 300 & & \\
\hline 19 & 0,9 & 1 & 2 & 2 & 248 & 437 & & \\
\hline 20 & 0,9 & 1 & 2 & 2 & 116 & 248 & & \\
\hline 21 & 0,9 & 1 & 2 & 6 & 115 & 178 & & \\
\hline
\end{tabular}


Saline monkey O

$\begin{array}{rrrrr}\text { no. } & \text { plexStart1 } & \text { plexEnd1 } & \text { plexStart2 } & \text { plexEnd2 } \\ 1 & 00: 15: 00 & 00: 25: 00 & 00: 58: 00 & 01: 08: 00 \\ 2 & 00: 16: 00 & 00: 26: 00 & & \\ 3 & 00: 40: 00 & 00: 50: 00 & & \\ 4 & 00: 17: 00 & 00: 27: 00 & & \\ 5 & 00: 35: 00 & 00: 45: 00 & & \\ 6 & 00: 28: 00 & 00: 38: 00 & & \\ 7 & 00: 14: 00 & 00: 24: 00 & & \\ 8 & 00: 22: 00 & 00: 32: 00 & & \\ 9 & 00: 17: 00 & 00: 32: 00 & & \\ 10 & 00: 12: 00 & 00: 22: 00 & 00: 45: 00 & 00: 55: 00 \\ 11 & 00: 10: 00 & 00: 20: 00 & 00: 42: 00 & 00: 57: 00 \\ 12 & 00: 13: 00 & 00: 21: 00 & & \\ 13 & 00: 17: 00 & 00: 27: 00 & & \\ 14 & 00: 14: 00 & 00: 19: 00 & & \\ 15 & 00: 15: 00 & 00: 25: 00 & & \\ 16 & 00: 11: 00 & 00: 21: 00 & & \\ 17 & 00: 15: 00 & 00: 25: 00 & & \\ 18 & 00: 15: 00 & 00: 25: 00 & & \end{array}$

\begin{tabular}{rrrrr} 
no. & plexStart1 & \multicolumn{1}{c}{ plexEnd1 } & plexStart2 & plexEnd2 \\
1 & $0: 55: 00$ & $01: 05: 00$ & & \\
2 & $00: 23$ & $0: 37: 00$ & & \\
3 & $00: 34$ & $0: 45: 00$ & & \\
4 & $00: 30$ & $0: 41: 00$ & & \\
5 & $00: 12: 00$ & $00: 22: 00$ & $00: 32: 00$ & $00: 39: 00$ \\
6 & $00: 25: 00$ & $00: 35: 00$ & $00: 59: 00$ & $01: 09: 00$ \\
7 & $00: 16: 00$ & $00: 26: 00$ & & \\
8 & $00: 12: 00$ & $00: 22: 00$ & $00: 45: 00$ & $00: 55: 00$ \\
9 & $00: 12: 00$ & $00: 26: 00$ & & \\
10 & $00: 30: 00$ & $00: 40: 00$ & & \\
11 & $00: 13: 30$ & $00: 23: 30$ & & \\
12 & $00: 15: 30$ & $0: 25: 00$ & & \\
13 & $00: 25: 00$ & $00: 35: 00$ & & \\
14 & $00: 09: 00$ & $00: 19: 00$ & & \\
15 & $00: 11: 00$ & $00: 21: 00$ & & \\
16 & $00: 14: 00$ & $00: 24: 00$ & & \\
17 & $00: 10: 00$ & $00: 20: 00$ & & \\
18 & $00: 15: 00$ & $00: 25: 00$ & & \\
19 & $00: 20: 00$ & $00: 35: 00$ & & \\
20 & $00: 09: 00$ & $00: 19: 00$ & & \\
21 & $00: 10: 00$ & $00: 20: 00$ & &
\end{tabular}




\section{Summary and Conclusion}

This thesis contributes to the understanding of spatial visual attention in the medial temporal area MT. The experiments described in the first part cover perceptual characteristics of spatial attention in humans, investigating hemifield dependence as well as the influence of signal strength on to the gradual deployment of spatial attention. In the second part, I aimed to shed light on the cholinergic involvement of attentional modulation on firing rate in macaque MT, performing single-cell recording while executing local neuropharmacological manipulations.

Spatial visual attention is a selective process, allowing subjects to prioritize spatial locations in the visual field and therefore enhance their processing. Physiologically, spatial attention leads to an increase in neuronal firing rate when the attentional focus matches a neuron's receptive field (Treue and Maunsell, 1999). Although our knowledge about visual spatial attention has advanced significantly during the last two decades and led to the abovementioned definitions, important pieces are still missing from a complete understanding. Spatial attention is typically investigated from two perspectives, behavioral and neurophysiological. Here, both of these approaches were used to allow broad insights into the characteristics of spatial visual attention. Additionally, pharmacological manipulations were conducted during neurophysiological measurements to investigate the cholinergic contribution to attention-induced change in neuronal firing rate.

The mid-level visual area MT is the cortical area of interest in this thesis. It serves as an ideal visual area to study visual spatial attention, as it is one of the best-understood areas in terms of its sensory properties, and is strongly influenced by top-down processes such as spatial attention. 
The first two manuscripts in this thesis cover perceptual aspects of spatial attention in humans, using linear motion stimuli to target area MT. The novel task design used in the two psychophysical studies allowed the measurement of pure spatial attention effects on the perception of two stimuli. The effect of graded attention was investigated, with the direction discrimination threshold for linear motion used as a measure of performance.

In the first study, I found that simultaneously attending to two linear motion stimuli did not seem to involve attentional cost when stimuli were distributed across the two hemifields. Splitting attention within one hemifield led to deterioration in performance. With this within-hemifield stimulus placement, decreasing the amount of spatial attention allocated to a stimulus led to a gradual decrement in performance. In the case of simple visual performance, such as direction discrimination, the tasks are seen to have low attentional cost (Braun and Julesz, 1998). It is consequently assumed that there is some amount of visual awareness outside the attentional spotlight, permitting the subject to perform a task with low attentional cost equally well for stimuli at locations inside and outside of the spotlight (Braun and Julesz, 1998). This theory does not seem to hold true when stimuli are distributed within one hemifield. Alternatively, this result could be explained by the existence of two parallel active attentional channels that independently process visual information. The results of this study extent the bilateral attentional advantage, as previously shown for higher-level tasks (Alvarez and Cavanagh, 2005,) as well as for elementary visual tasks (Reardon et al., 2009), to the linear motion discrimination task.

The second psychophysical study also supports the bilateral processing advantage for linear motion direction discrimination observed in the first study. Additionally, the signal strength of the target dot pattern was manipulated by varying the percentage of coherently moving dots. This allowed to equate the effects of attention with the effects of signal strength and to measure their 
individual as well as their interactive influence on perceptual performance. Spatial attention, when split or gradually diminished across both hemifields, did not significantly influence performance, whereas signal strength showed a strong and graded influence. As earlier behavioral studies revealed that attention enhances the subjective appearance of motion coherence (Liu et al., 2006), an interaction between the two factors was expected. However, no interaction of these two factors was found, suggesting that spatial attention and signal strength co-act as independent systems in the subjects' perception of linear motion when stimuli are distributed across both hemifields. As attentional capacity limits were possibly not reached with this across-hemifield task design, it would be of interest to investigate the interactive influence of signal strength and attentional deployment on behavioral performance when both stimuli are placed within one hemifield.

Additionally, it would be of particular interest whether the demonstrated hemifield dependence is even more prevalent in pathological conditions. It was shown that spatial attention performance is in general diminished in Alzheimer patients (Rizzo et al., 2000), including mechanisms of divided spatial attention (Parasurman and Haxby, 1993). But how is this pathologically diminished performance in attentional tasks correlated with anatomical or functional deficits? The cholinergic system is supposed to play a major regulative role in the context of visual attention. The cognitive deficits characterizing Dementia and Alzheimer's disease have been linked to a diminished amount of cholinergic fibers from basal forebrain - the main cholinergic source - to cortex and hippocampus, and an associated loss of cholinergic neurotransmission (Francis et al., 1999). Additionally, a strong relationship between specific cholinergic receptor-type expression and neuropathological key features was reported for Alzheimer's disease (Wevers et al., 2000). Although the relationship between perceptual deficits in spatial attention and a reduction of the cholinergic activity in visual cortex has been demonstrated, the precise nature of the cholinergic contribution to attentional modulation is still ambiguous. 
The aim of the second part of this thesis was to investigate whether there is an interactive effect of spatial attention and local cholinergic manipulations on neuronal response in area MT in the macaque monkey. The local neuropharmacological manipulation was performed using the method of pressure injection. The implementation of the pressure injection system was part of this thesis and is described in detail in manuscript three. Using this method, the area around the recording electrode could be modulated in a spatially local and temporally restricted fashion. This allowed to influence the local cholinergic system by either blocking the muscarinic or nicotinic receptor type, or enhancing the neuromodulator acetylcholine in the extracellular space. Manuscript four in this thesis is the first study describing a pharmacological manipulation in area MT of an awake, behaving monkey. I found evidence that the cholinergic system is involved in attentional modulation in area MT, as an increase in attentional modulation was observed when acetylcholine was injected. However, before a strong statement can be made, more data is needed to validate this effect. Although a strong influence of spatial attention on the neuronal firing rate was observed, the attentional modulation was only marginally diminished by injection of the muscarinic antagonist scopolamine for the full data set. When selecting only those cells whose firing rate was significantly influenced by scopolamine injection, it was shown that the blockage of the muscarinic receptor type in area MT also led to a significant increase in attentional modulation. In contrast, in area V1 of the macaque, a blockage of the muscarinic receptor type led to reduction in attentional modulation (Herrero et al., 2008). This diverse contribution of the same receptor types across visual areas supports an area-dependent change in neurotransmitter contribution on attentional modulation in the visual system (Disney et al., 2014). Visual area V1 has specific anatomical characteristics, such as a reduced amount of inhibitory neurons as well as an altered quantity of cholinergic receptor subtypes, that distinguishes it from other visual areas such as area MT (DeFelipe et al., 1999, Disney et al., 2006). This suggests a different role for cholinergic contribution, implying that area V1 is of limited suitability as a model for cholinergic modulation of visual cortical circuits in the macaque. 
It is of interest to bring the observed local cholinergic effect in area MT into the context of the global attention-induced cholinergic activity of the whole cortex. Top-down visual processes, such as spatial attention, have been shown to be predominantly mediated by the lateral prefrontal and the parietal cortex (Kastner \& Ungerleider, 2000). The prefrontal cortex is also seen as a central coordinating point of acetylcholine release, targeting the main cholinergic source for the cortex, the basal forebrain cholinergic system (Sarter et al., 2005). It is further proposed that prefrontal efferent projections either directly or indirectly target the sensory cortex, via the basal forebrain cholinergic system, thereby forming the 'top-down modulation of detection' (Sarter et al., 2005). Acetylcholine acts as a neuromodulator in the brain and is therefore seen to have limited influence on local and fast attention-mediated effects. An interaction with other neurotransmitter systems is therefore very likely. Glutamate is the major excitatory and fast-acting neurotransmitter in the brain (see for a review Meldrun, 2000). It is released from visual cortical neurons when they are visually stimulated (bottom-up process). It is proposed that this bottom-up process interacts with top-down influences such as spatial attention, mediated by cholinergic release. This synergistic effect is therefore proposed to lead to enhance signal detection ( $Y u$ and Dayan, 2002 and 2005). As well as the neurotransmitter glutamate, other neurotransmitters are also likely to be involved in attentional modulation, such as the inhibitory neurotransmitter $\gamma$ Aminobutyric acid (GABA). In area MT, it was demonstrated that muscarinic cholinergic receptors are located on GABA-ergic neurons, mediating inhibitory effects (Disney et al., 2012). These opposing cell- and receptor-specific effects lead to the assumption of a highly specialized neurotransmitter network in which the neuromodulator acetylcholine is embedded, leading to an optimal response enhancement in attentional conditions. Further pharmacological studies are consequently needed to clarify the involvement of acetylcholine in attentional modulation. 


\section{References}

Abrams J, Barbot A, Carrasco M (2010) Voluntary attention increases perceived spatial frequency. Atten Percept Psychophysics 72(6): 1510-1521.

Albright TD, Desimone R, Gross CG (1984) Columnar organization of directionally selective cells in visual area MT of the macaque. Journal of Neurophysiology 51(1): 16-31.

Albright TD (1984) Direction and orientation selectivity of neurons in visual area MT of the macaque. Journal of Neurophysiology 52(6): 1106-30.

Albright TD, Desimone R (1987) Local precision of visuotopic organization in the middle temporal area (MT) of the macaque. Exp Brain Res. 65(3): 582-92.

Alvarez GA, Cavanagh P (2005) Independent resources for attentional tracking in the left and right visual hemifields. Psychol Sci. 16(8): 637-43.

Anton-Erxleben K, Henrich C, Treue S (2007) Attention changes perceived size of moving visual patterns. Journal of Vision 7(11):5.1-9.

Anton-Erxleben K, Carrasco M (2013) Attentional enhancement of spatial resolution: linking behavioral and neurophysiological evidence. Nature Review Neuroscience 14(3):188-200.

Arnold HM, Burk JA, Hodgson EM, Sarter M, Bruno JP (2002) Differential cortical acetylcholine release in rats performing a sustained attention task versus behavioral control tasks that do not explicitly tax attention. Neuroscience 114:451-460.

Aston-Jones G, Shaver R, Dinan TG (1985) Nucleus basalis neurons exhibit axonal branching with decreased impulse conduction velocity in rat cerebrocortex. Brain Res. 325(1-2):271-85. 
Auld DS, Kornecook TJ, Bastianetto S, Quirion R (2002) Alzheimer's disease and the basal forebrain cholinergic system: relations to beta-amyloid peptides, cognition, and treatment strategies. Prog Neurobiol. 68(3):209-45.

Benagiano V, Virgintino D, Flace P, Girolamo F, Errede M, Roncali L, Ambrosi G (2003) Choline acetyltransferase-containing neurons in the human parietal neocortex. Eur J Histochem. 47(3):253-256.

Bentley P, Vuilleumier P, Thiel CM, Driver J, Dolan RJ (2003) Cholinergic enhancement modulates neural correlates of selective attention and emotional processing. Neuroimage 20(1):58-70.

Bigl V, Woolf NJ, Butcher LL (1982) Cholinergic projections from the basal forebrain to frontal, parietal, temporal, occipital, and cingulate cortices: a combined fluorescent tracer and acetylcholinesterase analysis. Brain Research Bulletin 8(6):727-49.

Boussaoud D, Ungerleider LG, Desimone R (1990) Pathways for motion analysis: cortical connections of the medial superior temporal and fundus of the superior temporal visual areas in the macaque. J Comp Neurol. 296(3):462-95.

Braun J, Julesz B (1998) Withdrawing attention at little or no cost: detection and discrimination tasks. Perception \& psychophysics 60:1-23.

Broadbent DA (1971) Decision and stress. London: Academic Press.

Butts DA, Goldman MS (2006) Tuning curves, neuronal variability, and sensory coding. PLoS Biology 4(4):e92.

Carrasco M, McElree B (2001) Covert attention accelerates the rate of visual information processing. Proc Natl Acad Sci USA 98(9):5363-7.

Carrasco M, Ling S, Read S (2004) Attention alters appearance. Nature Neuroscience 7(3):308-13. 
Carrasco, M (2006). Covert attention increases contrast sensitivity: Psychophysical, neurophysiological, and neuroimaging studies. In S. Martinez-Conde, S. L. Macknik, L. M. Martinez, J. M. Alonso, \& P. U. Tse (Eds.), Visual perception. Part I. Fundamentals of vision: Low and midlevel processes in percetion - Progress in Brain Research Amsterdam: Elsevier pp. 33-70.

Carrasco M (2011) Visual attention: the past 25 years. Vision Research 51(13):1484-525.

Castiello U, Umiltà C (1992) Splitting focal attention. Journal of Experimental Psychology: Human Perception and Performance 18(3):837-48.

Cavanagh P, Alvarez GA (2005) Tracking multiple targets with multifocal attention. Trends Cogn Sci. 9(7):349-54.

Changeux JP (2010) Allosteric receptors: from electric organ to cognition. Annu Rev Pharmacol Toxicol 50:1-38.

Chiba AA, Bushnell PJ, Oshiro WM, Gallagher M (1999) Selective removal of cholinergic neurons in the basal forebrain alters cued target detection. Neuroreport 10(14):3119-23.

Colby CL, Duhamel JR, Goldberg ME (1993) Ventral intraparietal area of the macaque: anatomical location and visual response properties. Journal of Neurophysiology 69:902-914.

Cornelissen FW, Peters EM, Palmer J (2002) The Eyelink Toolbox: eye tracking with MATLAB and the Psychophysics Toolbox. Behavior Research Methods 34(4):613-7.

Dale HH (1914) The action of certain ersters and ethers of choline and their relation to muscarie. The Journal of Pharmacology and Experimental Therapeutics 6:147-190.

Dale HH, Dudley HW (1929) The presence of histamine and acetylcholine in the spleen of the ox and horse. The Journal of Physiology 68:97-123. 
Dani JA, Bertrand D (2007) Nicotinic acetylcholine receptors and nicotinic cholinergic mechanisms of the central nervous system. Annu Rev Pharmacol Toxicol. 47:699-729.

DeFelipe J, González-Albo MC, Del Río MR, Elston GN (1999) Distribution and patterns of connectivity of interneurons containing calbindin, calretinin, and parvalbumin in visual areas of the occipital and temporal lobes of the macaque monkey. Journal of Comparable Neurology 412(3):515-26.

DeYoe EA, Van Essen DC (1988) Concurrent processing streams in monkey visual cortex Trends in neurosciences 11(5):219-226.

Disney AA, Domakonda KV, Aoki C (2006) Differential expression of muscarinic acetylcholine receptors across excitatory and inhibitory cells in visual cortical areas V1 and V2 of the macaque monkey. J Comp Neurol 499(1):49-63.

Disney AA1, Aoki C, Hawken MJ (2012) Cholinergic suppression of visual responses in primate $\mathrm{V} 1$ is mediated by GABAergic inhibition. $\mathrm{J}$ Neurophysiol. 108(7):1907-23.

Disney AA, Alasady HA, Reynolds JH (2014) Muscarinic acetylcholine receptors are expressed by most parvalbumin-immunoreactive neurons in area MT of the macaque. Brain and Behavior 4(3):431-45.

Dobkins KR, Albright TD (1994) What happens if it changes color when it moves?: the nature of chromatic input to macaque visual area MT. Journal of Neuroscience 14(8):4854-70.

Doig NM, Magill PJ, Apicella P, Bolam JP, Sharott A. (2014) Cortical and thalamic excitation mediate the multiphasic responses of striatal cholinergic interneurons to motivationally salient stimuli. J Neurosci. 34(8):3101-17. 
Dubner R, Zeki SM (1971) Response properties and receptive fields of cells in an anatomically defined region of the superior temporal sulcus in the monkey. Brain Research 35(2):528-32.

Duffy CJ, Wurtz RH (1991) Sensitivity of MST neurons to optic flow stimuli. I. A continuum of response selectivity to large-field stimuli. Journal of Neurophysiology 65:1329-1345.

Eckenstein F, Thoenen H (1983) Cholinergic neurons in the rat cerebral cortex demonstrated by immunohistochemical localization of choline acetyltransferase. Neurosci Lett 36:211-215.

Eckenstein F, Baughman RW (1984) Two types of cholinergic innervation in cortex, one co-localized with vasoactive intestinal polypeptide. Nature 309(5964):153-5.

Eriksen CW, St James JD (1986) Visual attention within and around the field of focal attention: a zoom lens model. Percept Psychophys. 40(4):225-40.

Everitt BJ, Robbins TW (1997) Central cholinergic systems and cognition. Annual Review of Psychology 48:649-684.

Fanini A, Assad JA (2009) Direction Selectivity of Neurons in the Macaque Lateral Intraparietal Area. Journal of Neurophysiology 101(1):289-305.

Fodale V, Mafrica F, Caminiti V, Grasso G (2006) The cholinergic system in Down's syndrome. Journal of Intellectual Disabilities 10(3):261-74.

Fortenbaugh FC, Prinzmetal W, Robertson LC (2011) Rapid changes in visualspatial attention distort object shape. Psychon Bull Review 18(2):287-94.

Francis PT, Palmer AM, Snape M, Wilcock GK (1999) The cholinergic hypothesis of Alzheimer's disease: a review of progress. J Neurol Neurosurg Psychiatry 66(2):137-47.

Gandhi SP, Heeger DJ, and Boynton GM (1999) Spatial Attention Affects Brain Activity in Human Primary Visual Cortex. Proc. Natl. Acad. Sci. 96:33143319 . 
Gattass R, Gross CG (1981) Visual topography of striate projection zone (MT) in posterior superior temporal sulcus of the macaque. J Neurophysiol. $46(3): 621-38$.

Geula C, Mesulam MM (1989) Cortical cholinergic fibers in aging and Alzheimer's disease: a morphometric study. Neuroscience 33(3):469-81.

Gross CG, Rocha Miranda CE, Bender DB (1972) Visual Properties Neurons in inferotemporal cortex of the macaque. J. Neurophysiol 35:96 -111.

Guillery RW, Feig SL, Lozsádi DA (1998) Paying attention to the thalamic reticular nucleus. Trends Neurosci. 21(1):28-32.

Hallanger AE, Levey AI, Lee HJ, Rye DB, Wainer BH (1987) The origins of cholinergic and other subcortical afferents to the thalamus in the rat. $\mathrm{J}$ Comp Neurol 262:105-124.

Hasselmo ME, Sarter M (2011) Modes and models of forebrain cholinergic neuromodulation of cognition. Neuropsychopharmacology 36(1):52-73.

Hasselmo ME, Stern CE (2006) Mechanisms underlying working memory for novel information. Trends Cogn Sci. 10(11):487-93.

Henderson Z (1981) A projection from acetylcholinesterase-containing neurones in the diagonal band to the occipital cortex of the rat. Neuroscience 6(6):1081-1088.

Herrmann K, Montaser-Kouhsari L, Carrasco M, Heeger DJ (2010) When size matters: attention affects performance by contrast or response gain. Nature Neuroscience 13:1554-1559

Herrero JL, Roberts MJ, Delicato LS, Gieselmann MA, Dayan P, Thiele A (2008) Acetylcholine contributes through muscarinic receptors to attentional modulation in V1. Nature 454(7208):1110-4. 
Hof PR, Morrison JH (1995) Neurofilament protein defines regional patterns of cortical organization in the macaque monkey visual system: a quantitative immunohistochemical analysis. Jornal of Comparative Neurology 352(2):161-86.

Houser CR, Crawford GD, Salvaterra PM, Vaughn JE (1985) Immunocytochemical localization of choline acetyltransferase in rat cerebral cortex: a study of cholinergic neurons and synapses. Journal of Comparative Neurology 234(1):17-34.

Hubel DH, Livingstone MS (1987) Segregation of form, color, and stereopsis in primate area 18. The Journal of Neuroscience 7(11):3378-415.

Ishii K, Kawachi T, Sasaki H, Kono AK, Fukuda T, Kojima Y (2005) Voxel-based morphometric comparison between early- and late-onset mild Alzheimer's disease and assessment of diagnostic performance of z score images. AJNR Am J Neuroradiol. 26(2):333-340.

Ishii M, Kurachi Y (2006) Muscarinic acetylcholine receptors. Curr Pharm Des. 12(28):3573-81.

Itti L, Koch C (2001) Computational modelling of visual attention. Nat Rev Neurosci. 2(3):194-203.

James W (1890) The Principles of Psychology, Vol. 1. Henry Holt and Company, New York, pp. 403-404.

Johnston MV, McKinney M, Coyle JT (1981) Neocortical cholinergic innervation: a description of extrinsic and intrinsic components in the rat. Experimental Brain Research 43(2):159-172.

Kahneman D (1973) Attention and effort. Englewood Cliffs, NJ: Prentice-Hall.

Kastner S, Ungerleider LG (2000) Mechanisms of visual attention in the human cortex. Annu Rev Neurosci. 23:315-341. 
Koch K, McLean J, Segev R, Freed MA, Berry MJ 2nd, Balasubramanian V, Sterling $\mathrm{P}$ (2006) How much the eye tells the brain. Current Biololgy 16(14):1428-34.

Koenigs M, Barbey AK, Postle BR, Grafman J (2009) Superior parietal cortex is critical for the manipulation of information in working memory. Journal of Neuroscience 29(47):14980-6.

Kramer AF, Hahn S (1995) Splitting the Beam: Distribution of Attention over Noncontiguous Regions of the Visual Field. Psychological Science $6(6): 381-386$.

Kumari V, Gray JA, Ffytche DH, Mitterschiffthaler MT, Das M, Zachariah E, Vythelingum GN, Williams SCR, Simmons A, Sharma T (2003) Cognitive effects of nicotine in humans: an fMRI study. Neuroimage 19:1002-1013.

Lisi M, Bonato M, Zorzi M (2015) Pupil dilation reveals top-down attentional load during spatial monitoring. Biol Psychol. 112:39-45

Liu AKL, Chang RCC, Pearce RKB, Gentleman SM (2015) Nucleus basalis of Meynert revisited: anatomy, history and differential involvement in Alzheimer's and Parkinson's disease. Acta Neuropathol. 129(4): 527540 .

Loewi O (1924) Über humorale Übertragbarkeit der Herznervenwirkung. Pflügers Archiv 189:239-242.

Loughlin SE, Foote SL, Bloom FE (1986) Efferent projections of nucleus locus coeruleus: topographic organization of cells of origin demonstrated by three-dimensional reconstruction. Neuroscience 18(2):291-306. 
Lukas RJ, Changeux JP, Le Novère N, Albuquerque EX, Balfour DJ, Berg DK, Bertrand D, Chiappinelli VA, Clarke PB, Collins AC, Dani JA, Grady SR, Kellar KJ, Lindstrom JM, Marks MJ, Quik M, Taylor PW, Wonnacott S (1999) International Union of Pharmacology. XX. Current status of the nomenclature for nicotinic acetylcholine receptors and their subunits. Pharmacol Rev. 51(2):397-401.

Luo TZ, Maunsell JH (2015) Neuronal Modulations in Visual Cortex Are Associated with Only One of Multiple Components of Attention. Neuron 86(5):1182-8.

Maunsell JHR, van Essen DC (1983) The connections of the middle temporal visual area (MT) and their relationship to a cortical hierarchy in the macaque monkey. The Journal of Neuroscience 3(12):2563-86.

Maunsell JHR, Cook EP (2002) The role of attention in visual processing. Philos. Trans. R. Soc. London Ser. B 357:1063-1072.

Martinez-Trujillo JC, Treue S (2002) Attentional Modulation Strength in Cortical Area MT Depends on Stimulus Contrast. Neuron 35:365-370.

Meldrum BS (2000) Glutamate as a neurotransmitter in the brain: review of physiology and pathology. J Nutr. 130(4S Suppl):1007S-15S.

McAdams C, Maunsell J (1999) Effects of attention on orientation-tuning functions of single neurons in macaque cortical area V4. Journal of Neuroscience 19(1):431-441.

McAlonan K, Cavanaugh J, Wurtz RH (2008) Guarding the gateway to cortex with attention in visual thalamus. Nature 456(7220):391-4.

McCormick DA, Prince DA (1985) Two types of muscarinic response to acetylcholine in mammalian cortical neurons. Proc Natl Acad Sci USA 82(18):6344-8. 
McGaughy J, Dalley JW, Morrison CH, Everitt BJ, Robbins TW (2002) Selective behavioral and neurochemical effects of cholinergic lesions produced by intrabasalis infusions of 192 IgG- saporin on attentional performance in a five-choice serial reaction time task. Journal of Neuroscience 22:19051913.

McGehee DS, Heath MJ, Gelber S, Devay P, Role LW (1995) Nicotine enhancement of fast excitatory synaptic transmission in CNS by presynaptic receptors. Science 269:1692-1696.

Mesulam MM, Mufson EJ, Wainer BH, Levey AI (1983a) Central cholinergic pathways in the rat: an overview based on an alternative nomenclature (Ch1-Ch6). Neuroscience 10(4):1185-1201.

Mesulam MM, Mufson EJ, Levey AI, Wainer BH (1983b). Cholinergic innervation of cortex by the basal forebrain: cytochemistry and cortical connections of the septal area, diagonal band nuclei, nucleus basalis (substantia innominata), and hypothalamus in the rhesus monkey. Journal of Comparative Neurology 214:170-197.

Mesulam MM, Mufson EJ (1984) Neural inputs into the nucleus basalis of the substantia innominata (Ch4) in the rhesus monkey. Brain 107:253-74.

Mesulam MM, Geula C (1988) Nucleus basalis (Ch4) and cortical cholinergic innervation in the human brain: observations based on the distribution of acetylcholinesterase and choline acetyltransferase. Journal of Comparative Neurology 275:216-240.

Mesulam MM (2013) Cholinergic Circuitry of the Human Nucleus Basalis and Its Fate in Alzheimer's Disease. J Comp Neurol. 521(18):4124-4144.

Mineur YS, Picciotto MR. (2010) Nicotine receptors and depression: revisiting and revising the cholinergic hypothesis. Trends Pharmacol Sci. 31(12):580-6. 
Mishkin M, Ungerleider LG (1982) Contribution of striate inputs to the visuospatial functions of parieto-preoccipital cortex in monkeys. Behavioural Brain Research 6(1):57-77.

Morawetz C, Holz P, Baudewig J, Treue S, Dechent P (2007) Split of attentional resources in human visual cortex. Vis Neurosci 24(6):817-26.

Movshon JA, Newsome WT (1996) Visual response properties of striate cortical neurons projecting to area MT in macaque monkeys. The Journal of Neuroscience 16(23):7733-41.

Nelson CL, Sarter M, Bruno JP (2005) Prefrontal cortical modulation of acetylcholine release in posterior parietal cortex. Neuroscience 132:347359 .

Newsome WT, Salzman CD (1993) The neuronal basis of motion perception. Ciba Found Symp. 174:217-30.

Noudoost B, Chang MH, Steinmetz NA, Moore T (2010) Top-down control of visual attention. Curr Opin Neurobiol. 20(2):183-190.

Orban GA, Lagae L, Raiguel S, Xiao D, Maes H (1995) The speed tuning of medial superior temporal (MST) cell responses to optic-flow components. Perception 24(3):269-85.

Parasurman R, Haxby JV (1993) Attention and brain function in Alzheimer's disease. Neuropsychology 7:242-272.

Parikh V, Kozak R, Martinez V, Sarter M (2007) Prefrontal acetylcholine release controls cue detection on multiple timescales. Neuron 56(1):141-54.

Parikh V, Sarter M (2008) Cholinergic mediation of attention: contributions of phasic and tonic increases in prefrontal cholinergic activity. Ann NY Acad Sci. 1129:225-35.

Parker AJ (2007). "Binocular depth perception and the cerebral cortex." Nature reviews. Neuroscience 8(5): 379-391. 
Pashler H (1994) Dual-task interference in simple tasks: data and theory. Psychol Bull. 116(2):220-44.

Pashler H, Johnston JC (1998) Attentional limitations in dual-task performance. In: Pashler H, editor. Attention. Hove (United Kingdom): Psychology Press pp. 155-189.

Passetti F, Dalley JW, O'Connell MT, Everitt BJ, Robbins TW (2002) Increased acetylcholine release in the rat medial prefrontal cortex during performance of a visual attentional task. European Journal of Neuroscience 12(8):3051-8.

Paul S, Jeon WK, Bizon JL, Han JS (2015) Interaction of basal forebrain cholinergic neurons with the glucocorticoid system in stress regulation and cognitive impairment. Front Aging Neurosci. 2:7-43.

Picciotto MR, Caldarone BJ, King SL, Zachariou V (2000) Nicotinic receptors in the brain. Links between molecular biology and behavior. Neuropsychopharmacology 22:451-465.

Picciotto MR, Higley MJ, Mineur YS (2012) Acetylcholine as a neuromodulator: cholinergic signaling shapes nervous system function and behavior. Neuron 76(1):116-29.

Ponce CR, Lomber SG, Born RT (2008) Integrating motion and depth via parallel pathways. Nature Neuroscience 11(2):216-23.

Posner MI (1980) Orienting of attention. Quarterly Journal of Experimental Psychology 32(1):3-25.

Posner MI, Snyder CR, Davidson BJ (1980) Attention and the detection of signals. Journal of Experimental Psychology 109(2):160-74.

Posner MI, Inhoff AW, Friedrich FJ, Cohen A (1987) Isolating Attentional Systems- a Cognitive-Anatomical Analysis. Psychobiology 15:107-121. 
Price JL , Amaral DG (1981) An autoradiographic study of the projections of the central nucleus of the monkey amygdala. The Journal of Neuroscience 1(11): 1242-1259.

Pylyshyn ZW, Storm RW (1988) Tracking multiple independent targets: evidence for a parallel tracking mechanism. Spatial Vision 3(3):179-97.

Reardon KM , Kelly JG, Matthews N (2009) Bilateral attentional advantage on elementary visual tasks. Vision Research 49(7): 691-701.

Reynolds JH, Chelazzi L (2004). Attentional modulation of visual processing. Annual Review of Neuroscience 27:611-647.

Reynolds JH, Pasternak T, Desimone R (2000) Attention increases sensitivity of V4 neurons. Neuron 26(3):703-14.

Rizzo M, Anderson SW, Dawson J, Myers R, Ball K (2000) Visual attention impairments in Alzheimer's disease. Neurology 54:1954-1959.

Rodman HR, Gross CG, Albright TD (1989) Afferent basis of visual response properties in area MT of the macaque. I. Effects of striate cortex removal. The Journal of Neuroscience 9(6):2033-50.

Rodman HR, Gross CG, Albright TD (1990) Afferent basis of visual response properties in area MT of the macaque. II. Effects of superior colliculus removal. The Journal of Neuroscience 10(4):1154-64.

Rosenberg A, Wallisch P, Bradley DC (2008) Responses to direction and transparent motion stimuli in area FST of the macaque. Visual Neuroscience 25(2):187-95.

Rubin RP (2007) A brief history of great discoveries in pharmacology: in celebration of the centennial anniversary of the founding of the American Society of Pharmacology and Experimental Therapeutics. Pharmacological Reviews 59(4):289-359. 
Runeson E, Boynton GM, Murray SO (2013) Effects of Task and Attentional Selection on Responses in Human Visual Cortex. Journal of Neurophysiology 109:2606-2617

Sarter M, Hasselmo ME, Bruno JP, Givens B (2005) Unraveling the attentional functions of cortical cholinergic inputs: interactions between signaldriven and cognitive modulation of signal detection. Brain Res Brain Res Rev. 48(1):98-111.

Saul AB, Cynader MS (1989) Adaptation in single units in visual cortex: the tuning of aftereffects in the spatial domain. Vis Neurosci. 2(6):593-607.

Skottun BC, Bradley A, Sclar G, Ohzawa I, Freeman RD (1987) The effects of contrast on visual orientation and spatial frequency discrimination: a comparison of single cells and behavior. J Neurophysiol. 57(3):773-86.

Soto D, Blanco MJ (2004) Spatial attention and object-based attention: a comparison within a single task. Vision Research 44(1):69-81.

Spitzer H, Desimone R, Moran J (1988) Increased attention enhances both behavioral and neuronal performance. Science 240(4850):338-40.

Tani M, Akashi N, Hori K, Konishi K, Kitajima Y, Tomioka H, Inamoto A, Hirata A, Tomita A, Koganemaru T, Takahashi A, Hachisu M (2015) Anticholinergic Activity and Schizophrenia. Neurodegenerative Disease 15(3):168-74.

Tatsuya H (1993) Molecular properties of muscarinic acetylcholine receptors. Proc Jpn Acad Ser B Phys Biol Sci. 89(6):226-256.

Taylor P, Radić Z (1994) The cholinesterases: from genes to proteins. Annu Rev Pharmacol Toxicol. 34:281-320.

Thiele A, Delicato LS, Roberts MJ, Gieselmann MA (2006) A novel electrodepipette design for simultaneous recording of extracellular spikes and iontophoretic drug application in awake behaving monkeys. J Neurosci Methods. 158(2):207-11. 
Thiele A, Herrero JL, Distler C, Hoffmann KP (2012) Contribution of cholinergic and GABAergic mechanisms to direction tuning, discriminability, response reliability, and neuronal rate correlations in macaque middle temporal area. Journal of Neuroscience 32(47):166o215 .

Tougu V (2001) Acetylcholinesterase: Mechanism of Catalysis and Inhibition. Current Medicinal Chemistry - Central Nervous System Agents 1(2):155170 .

Treue S, Maunsell JH (1996) Attentional modulation of visual motion processing in cortical areas MT and MST. Nature 382(6591):539-41.

Treue S, Maunsell JH (1999) Effects of attention on the processing of motion in macaque middle temporal and medial superior temporal visual cortical areas. The Journal of Neuroscience 19(17):7591-602.

Treue S, Martínez Trujillo JC (1999) Feature-based attention influences motion processing gain in macaque visual cortex. Nature 399(6736):575-9.

Treue S (2003) Visual attention: the where, what, how and why of saliency. Current Opinion in Neurobiology 13:428-432.

Treue S (2001) Neural correlates of attention in primate visual cortex. Trends Neurosci. 24(5):295-300.

Ungerleider LG, Desimone R (1986) Cortical connections of visual area MT in the macaque. J Comp Neurol. 248(2):190-222.

Ungerleider LG and Mishkin M (1982) Analysis of Visual Behavior (Ingle DJ, Goodale MA and Mansfield RJW eds), The MIT Press, Cambridge, MA pp. 549-586.

Van Bockstaele EJ, Biswas A, Pickel VM (1993) Topography of serotonin neurons in the dorsal raphe nucleus that send axon collaterals to the rat prefrontal cortex and nucleus accumbens. Brain Research 624(1-2):18898. 
Van Essen DC and Maunsell JH (1983) Hierarchical organization and functional streams in the visual cortex. Trends in Neurosciences 6:370-375.

von Engelhardt J, Eliava M, Meyer AH, Rozov A, Monyer H (2007) Functional characterization of intrinsic cholinergic interneurons in the cortex. The Journal of Neuroscience 27(21):5633-5642.

Wess J (2003) Novel insights into muscarinic acetylcholine receptor function using gene targeting technology. Trends Pharmacol Sci. 24(8):414-20.

Wevers A, Burghaus L, Moser N, Witter B, Steinlein OK, Schütz U, Achnitz B, Krempel U, Nowacki S, Pilz K, Stoodt J, Lindstrom J, De Vos RA, Jansen Steur EN, Schröder H (2000) Expression of nicotinic acetylcholine receptors in Alzheimer's disease: postmortem investigations and experimental approaches. Behav Brain Res. 113(1-2):207-15.

Whittaker VP (1988) The organization of the cholinergic synapse. Keio J Med. 37(3):234-54.

Yeshurun Y, Carrasco M (1998) Attention improves or impairs visual performance by enhancing spatial resolution. Nature 396(6706):72-75.

Yu AJ, Dayan P (2002) Acetylcholine in cortical inference. Neural Netw. 15(46):719-30.

Yu AJ, Dayan P (2005) Uncertainty, neuromodulation, and attention. Neuron 46(4):681-92.

Zeki SM (1974) Functional organization of a visual area in the posterior bank of the superior temporal sulcus of the rhesus monkey. The Journal of Physiology 236:549-573.

Zoli M, Jansson A, Sykova E, Agnati LF, Fuxe K (1999) Volume transmission in the CNS and its relevance for neuropsychopharmacology. Tr. Pharmacol Sci. 20:142-150. 


\section{Curriculum Vitae}

\section{PERSONAL INFORMATION}

Name:

Vera Katharina Veith, née Marks

Email:

vveith@dpz.eu

Date of birth:

01.03.1984

Place of birth:

Münster

\section{EDUCATION/ STUDY}

Since Feb 2012

Ph.D. student at the Georg August University Göttingen, member of the Center for Systems Neuroscience, Graduate School for Neurosciences, Biophysics, and Molecular Biosciences, Göttingen Germany,

Graduate student, working at the German Primate Center, Cognitive Neuroscience Laboratory, Göttingen, Germany

Advisor: Prof. Dr. Stefan Treue

Dec 2010-Feb 2012

Graduate assistant at the German Primate Center, Cognitive Neuroscience Laboratory, Göttingen, Germany

$2005-2010$

Diploma Study of Biology at the Johannes Gutenberg University Mainz, Germany

Degree: Dipl. Biol. („sehr gut“)

Dimploma Thesis: „Verhaltenspharmakologische Untersuchungen bezüglich $\mathrm{GABA}_{\mathrm{A}}$-Rezeptor vermittelte Effekte an Mäusen“, Klinik für Psychiatrie und Psychotherapie der Universitätsmedizin, Mainz

Advisor: Prof. Dr. Christa Neumeyer 
2004-2005

2004

2004
Study of Chemistry at the Ruhr University Bochum, Germany

Apprenticeship Paramedic, Die Johanniter, Münster, Germany

Degree: Certified Paramedic

Abitur, Anne-Frank-Gymnasium, Werne, Germany

TEACHING EXPERIENCE

Jul-Dec 2015

2014

2012-2014

Jul-Aug 2014

Jul-Sep 2012

2008-2009
Supervision of a $\mathrm{PhD}$ student

Tutorials on visual perception and attention in the Graduate Program Neurosciences, Georg August University Göttingen

Regular supervision of several neurobiology seminars, Georg August University Göttingen

Supervision of a Biology Bachelor student

Supervision of a Psychology Bachelor student

Research assistant at the Faculty of Biology/Zoology, Johannes-Gutenberg-University, Mainz 


\section{SELECTED COURSES/ WORKSHOPS/ INTERNSHIPS}

Feb 2015

Dec 2014

May 2014

Oct 2013

Oct 2013

Jun 2013

Mar 2013

Nov 2012

Oct 2012
Workshop „Global leadership in the knowledge society“, Graduate School for Neurosciences, Biophysics, and Molecular Biosciences (GGNB), Göttingen

Workshop „Scietific writing“, Graduate School for Neurosciences, Biophysics, and Molecular Biosciences (GGNB), Göttingen

Industry excursion to Quiagen, Graduate School for Neurosciences, Biophysics, and Molecular Biosciences (GGNB), Hilden

Course „Basic statistics for graduate students in the life science“, Department of medical statistics, Göttingen

NWG course „Analysis and Models in Neurophysiology“, Bernstein Center Freiburg

Workshop „Effective scientific communication“, Graduate School for Neurosciences, Biophysics, and Molecular Biosciences (GGNB), Göttingen

Workshop „Time Management in Doctoral Research. Aligning Time and Goals“, Graduate School for Neurosciences, Biophysics, and Molecular Biosciences (GGNB), Göttingen

Course „Introduction to animal experiment“, Graduate School for Neurosciences, Biophysics, and Molecular Biosciences (GGNB), Göttingen

Course „Seminar on good scientific practice“, Graduate School for Neurosciences, Biophysics, and Molecular Biosciences (GGNB), Göttingen 
Aug 2012

Apr 2011

Aug-Oct 2008
European Summer School „Visual Neuroscience from Spike to Awareness", Rauischholzhausen

Poster: Neuropharmacology of attentional modulation in primate visual cortex

Workshop "Kommunikationstraining für Wissenschaftler" by the Klaus Tschira Stiftung, German Primate Center, Göttingen

Internship, Bayer Crop Science, Frankfurt am Main, Germany

\section{CONFERENCE CONTRIBUTIONS}

Oct 2015

Mar 2015

Mar 2015

Nov 2014
Society for Neuroscience (SfN) Annual Meeting, Chicago, USA

Poster: Is spatial attentional gain modulation in area MT of primate visual cortex mediated by the cholinergic system?

Eleventh Goettingen Meeting of the German Society, Göttingen, Germany

Poster: Human linear visual motion direction discrimination thresholds: Effects of graded deployment of spatial attention

Primate Neurobiology Meeting, Göttingen, Germany

Poster: Cholinergic involvement in attentional modulation in area MT of primate visual cortex

Society for Neuroscience (SfN) Annual Meeting, Washington, USA

Poster 1: The role of the cholinergic system in attentional modulation in area MTof the primate visual cortex

Poster 2: The impact of graded spatial attention and motion coherence on human direction discrimination thresholds for linear visual motion 
Jul 2014

Mar 2014

May 2013

Mar 2013

Oct 2012

\section{PUBLICATIONS}

Published

Prepared for submission
Federation of European Neuroscience Societies (FENS) Meeting, Milano, Italy

Poster: Cholinergic modulation of attention effects in area MT of primate visual cortex

Primate Neurobiology Meeting, Tübingen, Germany

Poster: Cholinergic modulation of attention effects in area MT of primate visual cortex

Vision Sience Society (VSS) Annual Meeting, Naples, USA

Poster: The influence of graded spatial attention on human direction discrimination thresholds as a function of stimulus motion coherence

Tenth Goettingen Meeting of the German Society, Göttingen, Germany

Poster: Effects of graded spatial attention on human direction discrimination thresholds and their dependence on noise

Conference „Cellular Mechanisms of Sensory Processing“, Göttingen, Germany

Poster: Neuropharmacology of attentional modulation in primate visual cortex

A pressure injection system for investigating the neuropharmacology of information processing in awake behaving macaque monkey cortex. Veith VK, Quigley C, Treue S. J. Vis. Exp. (109), e53724, doi: 10.3791/53724 (2016).

Human linear visual motion direction discrimination thresholds: Graded deployment of spatial attention shows hemifield dependent resources. Veith VK, Treue S Journal of Vision 
Prepared for submission

Prepared for submission
Human linear visual motion direction discrimination thresholds: Effects of graded deployment of spatial attention and signal strength. Veith VK, Treue S

The role of the cholinergic system in attentional modulation in area MT of the primate visual cortex. Veith VK, Quigley C, Treue S 